\begin{tabular}{l|l|l} 
Universidad & Campus \\
de Excelencia \\
Politécnica \\
de Cartagena
\end{tabular}

\title{
ESTUDIO DE LOS PRINCIPALES COMPONENTES QUIIMICOS NO VOLÁTILES ASOCIADOS A LA CALIDAD DEL CACAO "NACIONAL" DE ECUADOR, COMO HERRAMIENTA EN LA CERTIFICACIÓN DE ORIGEN
}

\section{Programa de Doctorado:}

Técnicas Avanzadas de Investigación y Desarrollo Agrario y Alimentario

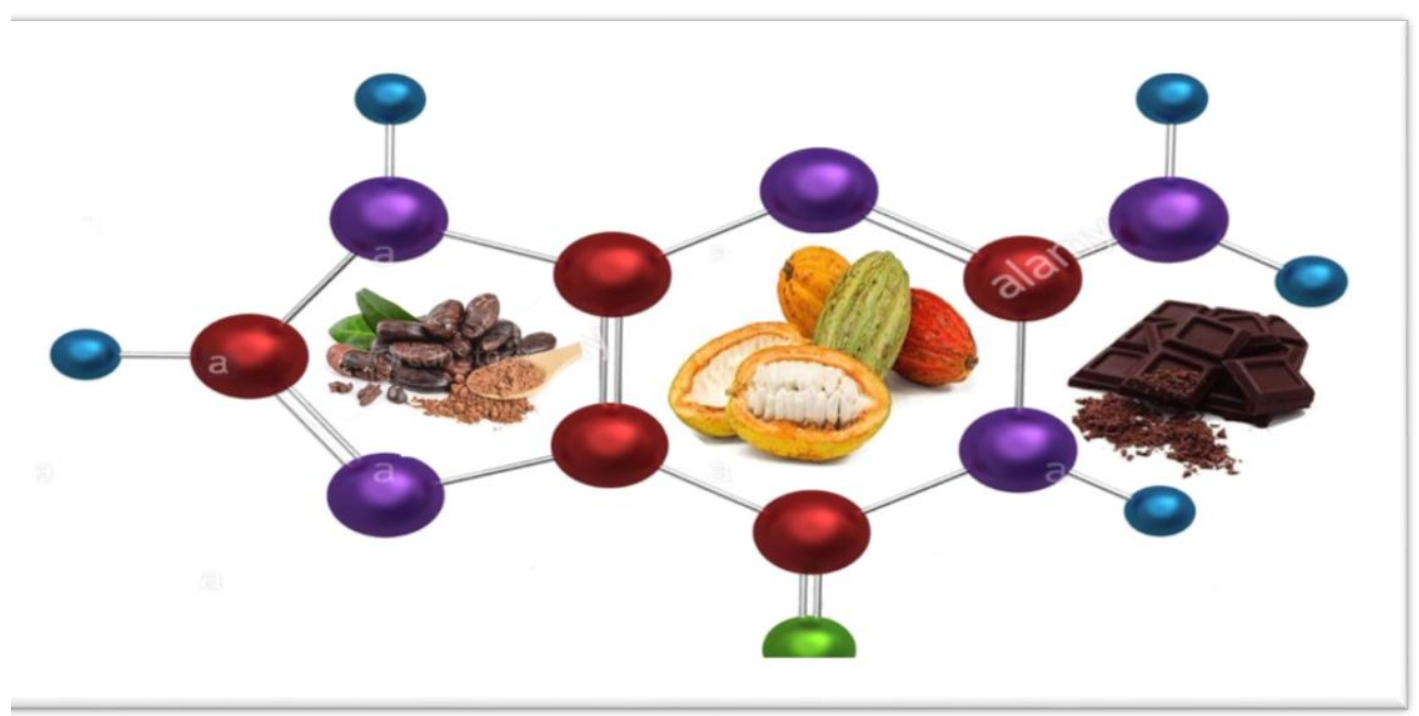

Autor: Iván Rodrigo Samaniego Maigua

Directores:

Dra. Cristina García-Viguera Dr. Pedro Miguel Mena Parreño

Tutor:

Dr. Pablo Fernández Escámez 


\section{Universidad \\ (3) Politécnica de Cartagena}

\section{Tesis Doctoral}

Estudio de los principales componentes químicos no volátiles asociados a la calidad del cacao "Nacional" de Ecuador, como herramienta en la certificación de origen

\section{Autor:}

Iván Rodrigo Samaniego Maigua

\section{Directores:}

Dra. Cristina García-Viguera

Dr. Pedro Miguel Mena Parreño

Tutor:

Dr. Pablo Fernández Escámez

\section{Cartagena, 2020}




\section{CONFORMIDAD DE SOLICITUD DE AUTORIZACIÓN DE DEPÓSITO DE}

\section{TESIS DOCTORAL POR EL/LA DIRECTOR/A DE LA TESIS}

Da. Cristina Garcia-Viguera y D. Pedro Miguel Mena Parreño, Directores de la Tesis doctoral: "Estudio de los principales Componentes Químicos No Volátiles asociados a la calidad del cacao Nacional de Ecuador, como herramienta en la certificación de origen".

\section{INFORMAN:}

Que la referida Tesis Doctoral, ha sido realizada por D. Iván Rodrigo Samaniego Maigua, dentro del Programa de Doctorado: Técnicas Avanzadas de Investigación y Desarrollo Agrario y Alimentario, dando su conformidad para que sea presentada ante el Comité de Dirección de la Escuela Internacional de Doctorado para ser autorizado su depósito.

Informe positivo sobre el plan de investigación y documento de actividades del doctorando/a emitido por el Director/ Tutor (RAPI).

La rama de conocimiento en la que esta tesis ha sido desarrollada es:

$\mathrm{x}$ Ciencias

Ciencias Sociales y Juridicas

Ingenieria y Arquitectura

En Cartagena, a 17 de septiembre del 2020

\section{LOS DIRECTORES DE LA TESIS}

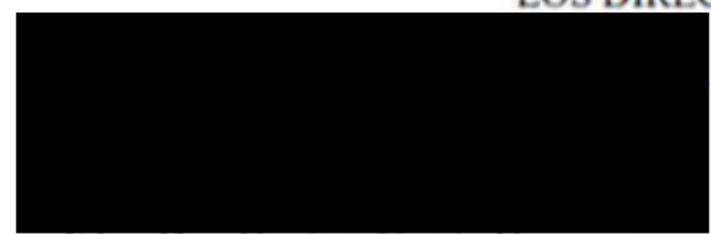

Fdo.: Dra. Cristina Garcia Viguera

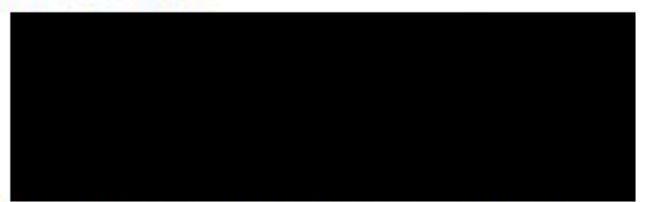

Fdo.: Dr. Pedro Miguel Mena Parreño 


\section{CONFORMIDAD DE DEPÓSITO DE TESIS DOCTORAL POR LA COMISIÓN ACADÉMICA DEL PROGRAMA}

D. Francisco Artés Hernández Presidente, de la Comisión Académica del Programa Técnicas Avanzadas en Investigación y Desarrollo Agrario y Alimentario".

\section{INFORMA:}

Que la Tesis Doctoral titulada, "Estudio de los principales Componentes Químicos No Volátiles asociados a la calidad del cacao Nacional de Ecuador, como herramienta en la certificación de origen", ha sido realizada, dentro del mencionado Programa de Doctorado, por D, Iván Rodrigo Samaniego Maigua, bajo la dirección y supervisión de la Dra. Cristina García-Viguera y Dr. Pedro Miguel Mena Parreño.

En reunión de la Comisión Académica, visto que en la misma se acreditan los indicios de calidad correspondientes y la autorización del Director/a de la misma, se acordó dar la conformidad, con la finalidad de que sea autorizado su depósito por el Comité de Dirección de la Escuela Internacional de Doctorado.

X Evaluación positiva del plan de investigación y documento de actividades por el Presidente de la Comisión Académica del programa (RAPI).

La Rama de conocimiento por la que esta tesis ha sido desarrollada es:

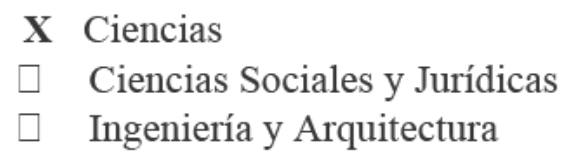

En Cartagena, a 18 de septiembre de 2020

EL PRESIDENTE DE LA COMISIÓN ACADÉMICA

Fdo:

FRANCISCO DE

ASIS|ARTES|

HERNANDEZ 


\section{DEDICATORIA}

A Dios por llenar mi camino de bendiciones

A mis padres, Marcial y Elena que con su ejemplo de humildad y sacrificio guiaron mi camino para culminar con éxito mi etapa de formación profesional

A mi esposa, Jimena por su apoyo decidido y comprensión en todo momento

A mis hijos, David y Danna porque son el motor que me da fuerza día a día para seguir adelante 


\section{AGRADECIMIENTOS}

Al Instituto Nacional de Investigaciones Agropecuarias (INIAP) y la Secretaria Nacional de Ciencia y Tecnología (SENECYT) en Ecuador, instituciones que brindaron el financiamiento para la realización del presente proyecto de investigación

Deseo expresar mi más profundo agradecimiento a la Dra. Cristina García-Viguera, quien desde el primer momento me brindó su apoyo técnico, consejos y ayuda incondicional para la realización de esta tesis doctoral. Realmente estoy orgulloso de haber compartido todo este tiempo con una investigadora de su nivel.

Al Dr. Pedro Mena, por su asesoramiento y apoyo científico durante la realización de este trabajo, su aporte ha constituido un pilar muy importante para culminar con éxito esta etapa de formación profesional. Trabajar con usted ha sido una experiencia invaluable.

Un reconocimiento especial a la Quím. Ms. Susana Espín e Ing. James Quiroz, con quienes hemos formado el grupo de investigación de cacao en Ecuador, gracias por su aporte profesional en el diseño y ejecución del trabajo en campo y laboratorio, así como por brindarme su amistad y confianza durante todo este tiempo de trabajo.

A Beatriz, Elena, Bladimir, Carmen, Rocío, Verónica, Soraya, compañeros del Departamento de Nutrición y Calidad del INIAP, con quienes hemos compartido alegrías y tristezas durante este tiempo, su apoyo ha sido muy importante para llevar a cabo este trabajo de investigación. 


\section{PRODUCCIÓN CIENTÍFICA}

\section{PUBLICACIONES EN REVISTAS CIENTÍFICAS}

Las publicaciones en revistas científicas generadas por la presente tesis doctoral son:

Publicación 1:

Título: $\quad$ Effect of the growing area on the methylxanthines and flavan-3-ols content in cocoa beans from Ecuador.

Autores: $\quad$ Iván Samaniego, Susana Espín, James Quiroz, Bladimir Ortiz, Wilman Carrillo, Cristina García-Viguera, Pedro Mena.

Revista: Journal of Food Composition and Analysis

Publicación Vol. 88(2020) 1034-48

DOI: $\quad$ https://doi.org/10.1016/j.jfca.2020.103448.

Publicación 2:

Título: $\quad$ How does the growing area condition the fat content and the fatty acid composition of Ecuadorian cocoa beans?

Autores: Iván Samaniego, Susana Espín, James Quiroz, Carmen Rosales, Wilman Carrillo, Pedro Mena, Cristina García-Viguera,

Revista: Journal of Food Sciences and Nutrition

Publicación En revisión

DOI: En revisión 


\section{PUBLICACIONES EN CONGRESOS}

La difusión del trabajo de investigación científica realizada en la tesis doctoral ha sido presentada en congresos y seminarios como:

Ponencia 1:

Congreso

Workshop de Investigación Agroalimentaria (WIA)

Título:

Estudio de los principales Componentes Químicos No Volátiles, asociados a la calidad del cacao de Ecuador, como herramienta en la certificación de origen

Autores: Iván Samaniego; Susana Espín; James Quiroz; Bladimir Ortiz; Pedro Mena; Cristina García-Viguera

Tipo de participación: Póster

Lugar de celebración: Cartagena (España)

Fecha $\quad 7-8$ de mayo del 2018

Ponencia 2:

Congreso

II International Conference on Food Science: Chapter Science and Entrepreneurship - INCOFS 2019

Título: $\quad$ El cacao: Fuente de Compuestos Bioactivos

Autores: Iván Samaniego; Susana Espín; James Quiroz; Pedro Mena, Cristina García-Viguera

Tipo de participación: Oral

Lugar de celebración: Quito (Ecuador)

Fecha 2-4 de diciembre del 2019 
Ponencia 3:

Congreso I Congreso Internacional de Ciencia y Tecnología Agropecuaria

Título: $\quad$ Caracterización del perfil de Polifenoles en cacao CCN 51

Autores: Iván Samaniego; Susana Espín; James Quiroz; José Luis Yépez; Pedro Mena, Cristina García-Viguera

Tipo de participación: Oral

Lugar de celebración: Quito (Ecuador)

Fecha $\quad 13-15$ de junio del 2018

\section{Ponencia 4:}

Congreso

I Congreso Internacional de Ciencia de los Alimentos y Biotecnología

Título: $\quad$ Caracterización de Metilxantinas en cacao CCN 51

Autores: Iván Samaniego; Susana Espín; Pedro Mena, Cristina García-Viguera

Tipo de participación: Oral

Lugar de celebración: Ambato (Ecuador)

Fecha 25-29 de junio del 2018 
Ponencia 5:

Congreso

I Simposio Internacional de Innovaciones Tecnológicas para Fortalecer la Cadena del Cacao en la Amazonía Ecuatoriana

Título:

Los Polifenoles en el Cacao Ecuatoriano

Autores:

Iván Samaniego; Susana Espín; James Quiroz; Pedro Mena, Cristina García-Viguera.

Tipo de participación: Oral

Lugar de celebración: Orellana (Ecuador)

Fecha 8-10 de julio del 2019 


\section{RESUMEN}

El objetivo de la presente tesis doctoral fue caracterizar los principales componentes químicos no volátiles, y el efecto de la zona geográfica de producción, sobre el contenido de estos fitoquímicos, en cacao Nacional ecuatoriano, como herramienta objetiva de calidad. De este modo, considerando que el cacao es uno de los principales productos de exportación del Ecuador, el presente trabajo podría servir para establecer nuevos parámetros para evaluar la calidad, certificar el origen y agregar valor al cacao ecuatoriano.

Para lograr este objetivo, en primer lugar, se estableció un plan de muestreo, seleccionando las tres provincias de mayor producción de cacao Nacional en la región Litoral (Costa del Pacífico) y en la región Amazónica del Ecuador. En cada provincia se seleccionaron 3 cantones y en cada cantón 5 fincas de productores, tomándose un total de 85 muestras por duplicado. Los análisis se realizaron utilizando los métodos estandarizados en el laboratorio de Servicio de Análisis e Investigación en Alimentos (LSAIA) del Instituto Nacional de Investigaciones Agropecuarias (INIAP) en Ecuador.

Una vez obtenidas las muestras, se procedió a determinar el contenido de metilxantinas (teobromina y cafeína) y el ratio de las mismas. Esta caracterización permitió establecer que el cacao Nacional ecuatoriano presenta mayores contenidos de teobromina, menores contenidos de cafeína y una relación teobromina/cafeína que varía en el rango de 5.2 a 7.6. Dicha relación permitió clasificar a esta variedad de cacao dentro del grupo de cacao tipo Trinitario. Todo ello demostró que los materiales ecuatorianos son "Finos", puesto que comercialmente, a nivel mundial, se reconoce a los cacaos de tipo Trinitario y Criollo, como materiales para elaboración de chocolates "Finos". El contenido en estas metilxantinas fue mayor en las muestras recogidas en la región amazónica.

Posteriormente, se procedió a la determinación del contenido total en compuestos fenólicos y el contenido de los principales flavan-3-oles presentes en el cacao Nacional ecuatoriano. Los resultados demostraron que esta variedad de cacao presenta contenidos de compuestos fenólicos totales en el rango de $33.55 \pm 5.74$ a $71.66 \pm 3.94$ mg GAE/g DW. Adicionalmente, su perfil de compuestos fenólicos presenta altos contenidos de (-)-epicatequina, seguido de (+)-catequina y procianidina B2, así como menores concentraciones de procianidina C1. Lo cual confirma que el cacao Nacional 
ecuatoriano, presenta contenidos apreciables de compuestos bioactivos, los mismos que influyen en sus características organolépticas, color y astringencia. Por otro lado, diferentes zonas de producción de cacao Nacional, se relacionaron con diferencias estadísticas significativas, en la concentración de estos compuestos.

Finalmente, se caracterizó el contenido de grasa y el perfil de ácidos grasos, demostrándose que esta variedad especial de cacao presenta contenidos de grasa total que varían en el rango de $45.61 \pm 1.27$ a $52.13 \pm 0.58 \mathrm{~g} / 100 \mathrm{~g} \mathrm{DW}$. Estos resultados permitieron establecer que el material procedente de la región Amazónica, presenta mayores contenidos de grasa total con relación a los procedentes de la región Costa del Pacífico; lo cual permite otorgar un valor añadido al cacao de esta zona geográfica de producción y mayor precio en el mercado, por su mayor rendimiento en peso para la fabricación de chocolate. Además, se determinó que el 97,7\% del perfil de ácidos grasos de esta variedad de cacao está compuesto de cuatros ácidos grasos (ácido palmítico $\mathrm{C} 16: 0$, ácido esteárico $\mathrm{C} 18: 0$, ácido oleico C18:1 y ácido linoleico C18:2), lo cual le confiere características diferentes con relación a las otras grasas vegetales.

En general, la presente tesis doctoral aportó el desarrollo de una herramienta objetiva de calidad inédita para productores, exportadores e industriales ecuatorianos, así como el establecimiento de mapas característicos en función de estos atributos químicos, con los cuales se puede identificar zonas de producción de cacao Nacional con características definidas, que permiten certificar el origen y mejorar los precios en el mercado internacional. 


\section{ABSTRACT}

The objective of this doctoral thesis was to characterize the main non-volatile chemical components and the effect of the geographical area of production on the content of these phytochemicals in Ecuadorian Nacional cocoa, as an objective quality tool. In this way, considering that cocoa is one of the main export products from Ecuador, the present work could serve to establish new parameters to evaluate the quality, certify the origin and add value to the Ecuadorian cocoa.

To achieve this objective, in the first place, a sampling plan was established, selecting the three provinces with the highest production of National cocoa in the Litoral region (Pacific Coast) and in the Amazon region of Ecuador. In each province, three cantons were selected and in each canton five producer farms, taking a total of 85 samples in duplicate. The analysis was carried out using standardized methods in the Food Analysis and Research Service Laboratory (LSAIA) of the National Institute of Agricultural Research (INIAP) in Ecuador.

Once the samples were obtained, the content of methylxanthines (theobromine and caffeine) and their ratio were determined. This characterization allowed to establish that the Ecuadorian Nacional cocoa has higher theobromine contents, lower caffeine contents and a theobromine/caffeine ratio that varies in the range of 5.2 to 7.6. This relationship allowed classifying this variety of cocoa within the group of Trinitario type cocoa, proving that the Ecuadorian materials are fine. Trinitario and Criollo type cocoas are recognized commercially worldwide as materials for making "Fine" chocolates. The content of these methylxanthines was higher in the samples collected in the Amazon region.

Subsequently, the total content of phenolic compounds and the content of the main flavan-3-ols present in Ecuadorian Nacional cocoa were determined. The results showed that this variety of cocoa present contents of total phenolic compounds in the range of $33.55 \pm 5.74-71.66 \pm 3.94 \mathrm{mg} \mathrm{GAE} / \mathrm{g} \mathrm{DW}$. Additionally, its profile of phenolic compounds presents high contents of (-)-epicatechin, followed by (+)-catechin and procyanidin $\mathrm{B} 2$, as well as lower concentrations of procyanidin $\mathrm{C} 1$. This confirms that the Ecuadorian Nacional cocoa present appreciable contents of bioactive compounds, the same ones that influence its organoleptic characteristics, color and astringency. On 
the other hand, different Nacional cocoa production areas were related to statistically significant differences in the concentration of these compounds.

Finally, the fat content and the fatty acid profile were characterized; demonstrating that this special variety of cocoa has total fat contents that vary in the range of $45.61 \pm 1.27$ to $52.13 \pm 0.58 \mathrm{~g} / 100 \mathrm{~g} \mathrm{DW}$. These results made possible to establish that cocoa from the Amazon region has a higher total fat content compared to those from the Pacific Coast region; this study allows adding value to the cocoa for its geographical area of production and a higher price in the market. The yield in weight is higher for the manufacture of chocolate. In addition, it was determined that $97.7 \%$ of the fatty acid profile of this variety of cocoa is composed of four fatty acids (palmitic acid C16:0, stearic acid C18:0, oleic acid C18:1 and linoleic acid C18:2), which gives it different characteristics compared to other vegetable fats.

In general, this doctoral thesis contributed to the development of an objective tool of unpublished quality for Ecuadorian producers, exporters and industrialists, as well as the establishment of characteristic maps based on these chemical attributes, with which National cocoa production zones can be identified with defined characteristics. This study will allow certifying the origin and improving prices in the international market. 


\section{ÍNDICE GENERAL}

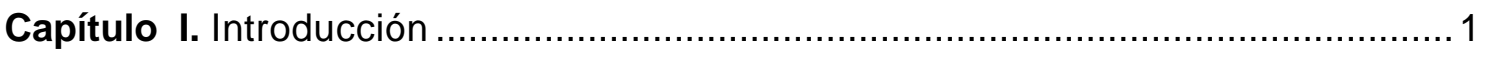

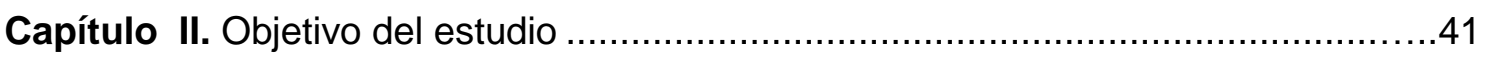

Capítulo III. Publicaciones desarrolladas en la presente Tesis Doctoral.................... 43

Capítulo IV. Discución general de resultados ................................................. 101

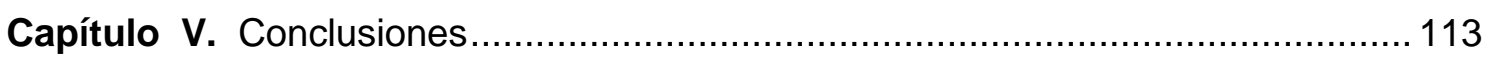

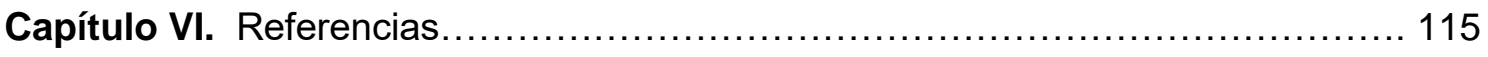




\section{ÍNDICE DE CONTENIDOS}

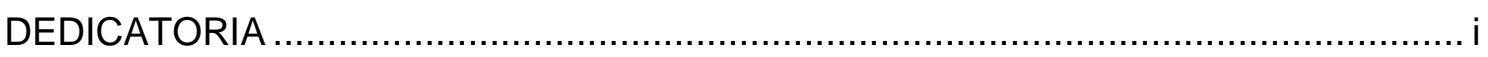

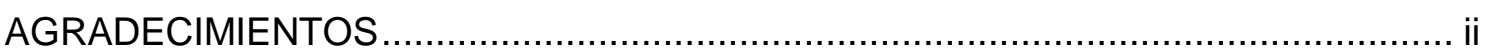

PRODUCCIÓN CIENTÍFICA...........................................................................ii

PUBLICACIONES EN REVISTAS CIENTÍFICAS ................................................iii

PUBLICACIONES EN CONGRESOS ............................................................. iv

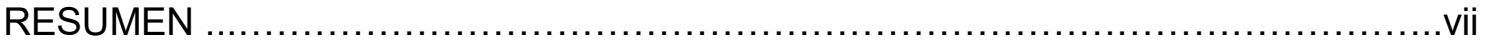

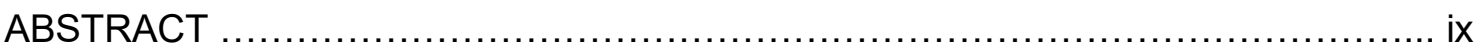

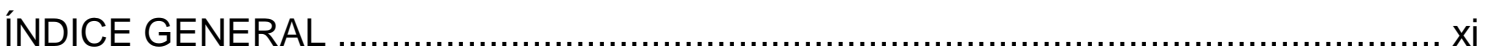

ÍNDICE DE CONTENIDOS ..............................................................................

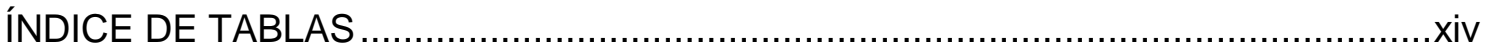

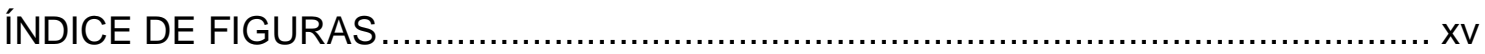

ÍNDICE DE ABREVIATURAS ........................................................................

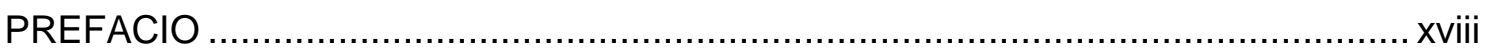

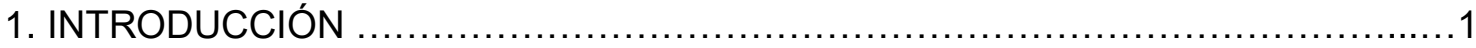

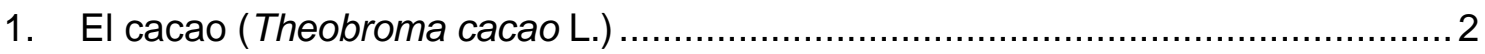

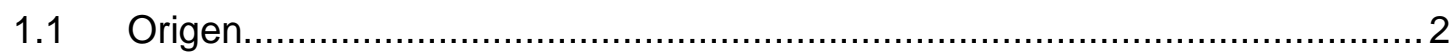

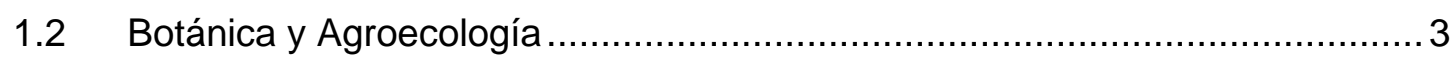

1.3 Cultivares de cacao y perfiles de sabor .................................................. 4

1.4 Historia del cultivo de cacao en Ecuador ..................................................... 6

1.5 Importancia socioeconómica del cacao en Ecuador ...................................... 9

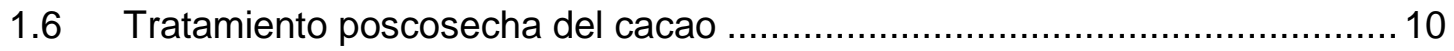

1.6.1 Fermentación.............................................................................. 10

1.6.1.1 Métodos de fermentación..................................................

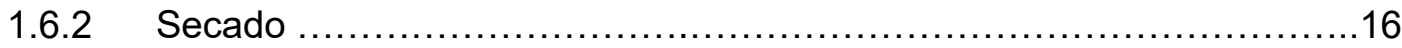

$1.7 \quad$ Atributos de calidad y composición química del cacao ................................18 


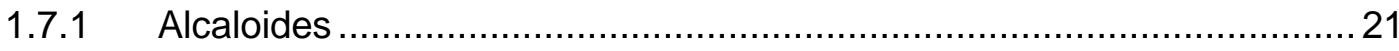

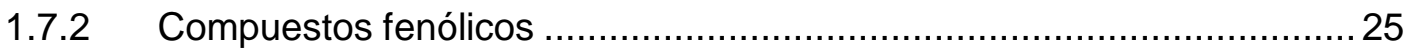

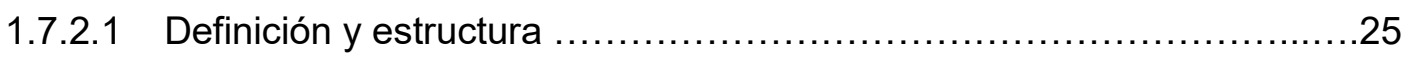

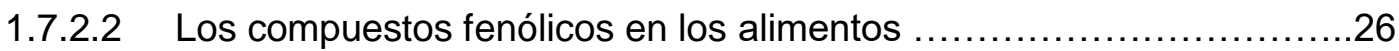

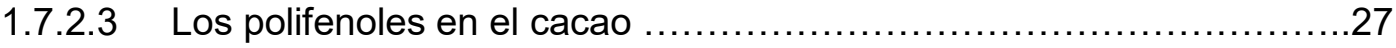

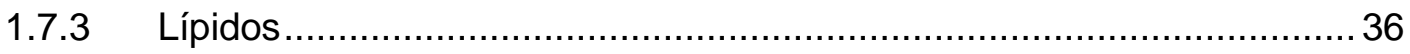

1.8 Situación actual de la calidad del cacao "Nacional" ecuatoriano .................... 40

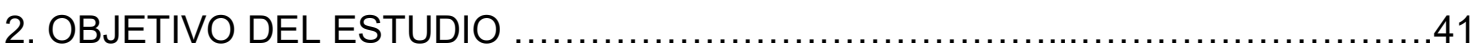

3. PUBLICACIONES DESARROLLADAS EN LA PRESENTE TESIS DOCTORAL ...43

4. DISCUCIÓN GENERAL DE RESULTADOS ...................................101

4.1 Metilxantinas y compuestos fenólicos en cacao "Nacional" ecuatoriano (Resultados relevantes de la Publicación 1) ..................................103

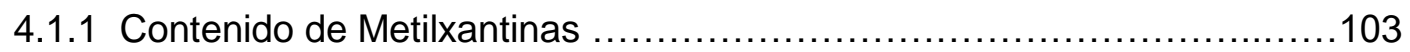

4.1.2 Contenido de compuestos fenólicos .........................................104

4.1.3 Contenido de Flavan-3-ols monómeros y procianidinas ....................105

4.2 Contenido de grasa y perfil de ácidos grasos en cacao "Nacional" ecuatoriano (Resultados relevantes de la Publicación 2) ...................................107

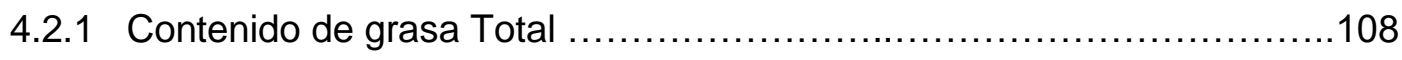

4.2.2 Contenido de Ácidos Grasos .............................................

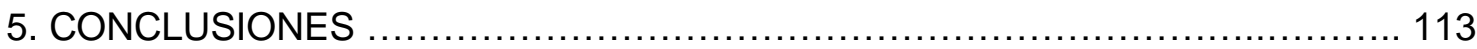

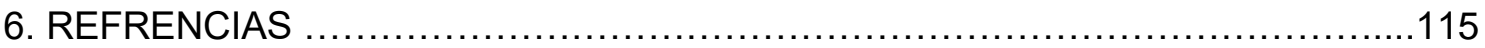




\section{ÍNDICE DE TABLAS}

Tabla 1. Clasificación taxonómica del cacao

Tabla 2. Requisitos de calidad del cacao beneficiado. 19

Tabla 3. Composición química nutricional del grano de cacao beneficiado 20

Tabla 4. Información resumida de alcaloides, contenido de teobromina y cafeína en cacao seco.

Tabla 5. Contenido de polifenoles en cacao seco de diferentes orígenes (Parte 1) .... 30

Tabla 6. Información resumida del contenido de grasa en granos de cacao seco ......37

Tabla 7. Perfil de ácidos grasos y triglicéridos en grasa de cacao ............................. 38

Tabla 8. Principales ácidos grasos en cacao de diferentes países 39 


\section{ÍNDICE DE FIGURAS}

Figura 1. Árbol de Theobroma cacao ................................................................. 4

Figura 2. Variedades de cacao a nivel mundial: Criollo $(A)$, Forastero $(B)$, Trinitario(C),

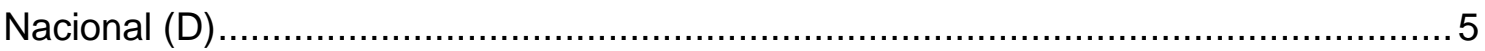

Figura 3. Fermentación de cacao en cajones de madera ........................................ 14

Figura 4. Fermentación de cacao en montones .................................................... 15

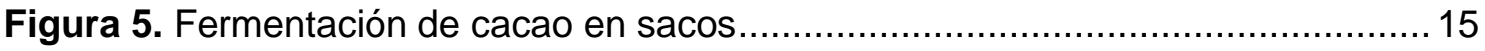

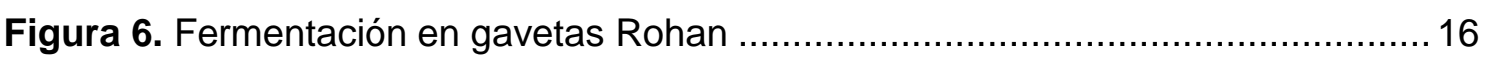

Figura 7. Secado natural de cacao sobre tendales de cemento (A) y en marquesinas (B)

Figura 8. Estructura química de las metilxantinas. Teobromina (A), Cafeína (B), Teofilina (C).

Figura 9. Efecto del proceso de fermentación sobre el contenido de teobromina en muestras de clones de cacao Nacional y fincas comerciales del Ecuador. 24

Figura 10. Producción de flavonoides y estilbenos a partir de Cumaril-CoA y de Malonil-CoA. 26

Figura 11. Estructura química de Flavan-3,4-diols del cacao: $(+)$ Catequina $(A),(+)$ Epicatequina (B), (+) Galocatequina (C), (-)-Epigalocatequina (D) 28

Figura 12. Principales Proantocianidinas del cacao: Procianidina B5 (A), Procianidina C1 (B) y Procianidina D (C) 29

Figura 13. Efecto de la fermentación sobre el contenido de polifenoles en cacao de diferentes orígenes.

Figura 14. Efecto de la fermentación sobre el contenido de (-)- epicatequina y proantocianidinas $B_{1}$ y $B_{2}$ del cacao.

Figura 15. Contenido promedio por provincia de TPC en cacao Nacional ecuatoriano. 105

Figura 16. Contenido promedio por provincia de (-)-epicatequina, (+)-catequina, Procianidina B2 y C1 en cacao Nacional ecuatoriano............................... 106

Figura 17. Chocolate negro rico en antioxidantes .107 
Figura 18. Contenido promedio por provincia de grasa total en cacao Nacional

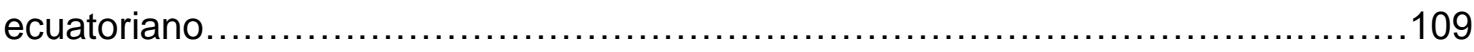

Figura 19. Composición porcentual de ácido grasos en cacao "Nacional"

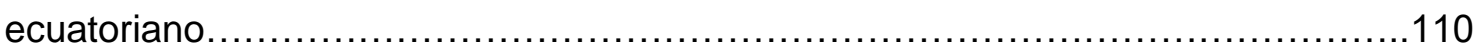




\title{
ÍNDICE DE ABREVIATURAS
}

\author{
CAT \\ (+)-Catequina \\ CAF \\ Cafeína \\ CF \\ Grasa de cacao \\ DW \\ Peso seco \\ EPI \\ (-)-Epicatequina \\ ESPAC \\ Encuesta de Superficie y Producción Agropecuaria Continua \\ FA \\ Ácidos grasos \\ GAE \\ Acido Gálico \\ GC-FID \\ Cromatografo de Gases acoplada a detector de lonización de \\ Flama \\ HDL \\ Lipoproteínas de Alta Densidad \\ HPLC-DAD \\ Cromatografo Líquido de Alta Resolución acoplado a detector de \\ Arreglo de Diodos \\ INEN \\ Servicio Ecuatoriano de Normalización \\ INIAP \\ Instituto Nacional de Investigaciones Agropecuarias del Ecuador \\ LDL \\ Lipoproteínas de baja densidad \\ MUFA \\ Ácidos grasos Mono insaturados \\ PC \\ Componente Principal \\ PCA \\ Análisis de Componentes Principales \\ PC-B2 \\ Procianidina B2 \\ PC-C1 \\ Procianidina C1 \\ PUFA \\ Ácidos grasos Poliinsaturados \\ SFA \\ Ácidos grados saturados \\ TBR \\ Teobromina \\ TBR/CAF \\ Relación Teobromina/Cafeína \\ TF \\ Grasa Total \\ TPC \\ Contenido de Polifenoles Totales




\section{PREFACIO}

La presente tesis Doctoral, titulada "Estudio de los principales componentes químicos no volátiles asociados a la calidad del cacao "Nacional" de Ecuador, como herramienta en la certificación de origen", se ha elaborado de acuerdo a la normativa de la UPCT para la presentación de tesis doctorales con un conjunto de publicaciones. Dicha tesis doctoral se articula en las siguientes partes:

- Resumen o Abstract, en donde se presenta la relevancia del trabajo de investigación.

- Una introducción (Capítulo I), que presenta los antecedentes bibliográficos más relevantes

- Los objetivos planteados en la investigación (Capitulo II)

- Una sección donde se recopila las publicaciones científicas obtenidas durante la investigación y que forman parte de la tesis doctoral (Capítulo III)

- Una sección que incluye los resultados más sobresalientes generados, así como una discusión general de los mismos (Capítulo IV)

- Las conclusiones generales del trabajo realizado (Capítulo V)

- Un capítulo final (Capítulo VI), que recoge la bibliografía utilizada en la introducción (Capítulo I) y en la discusión general de resultados (Capítulo IV)

La presente tesis doctoral no incluye una sección de "Materiales y Métodos", puesto que, esta información se encuentra redactada en las publicaciones científicas que se generaron durante la investigación. 


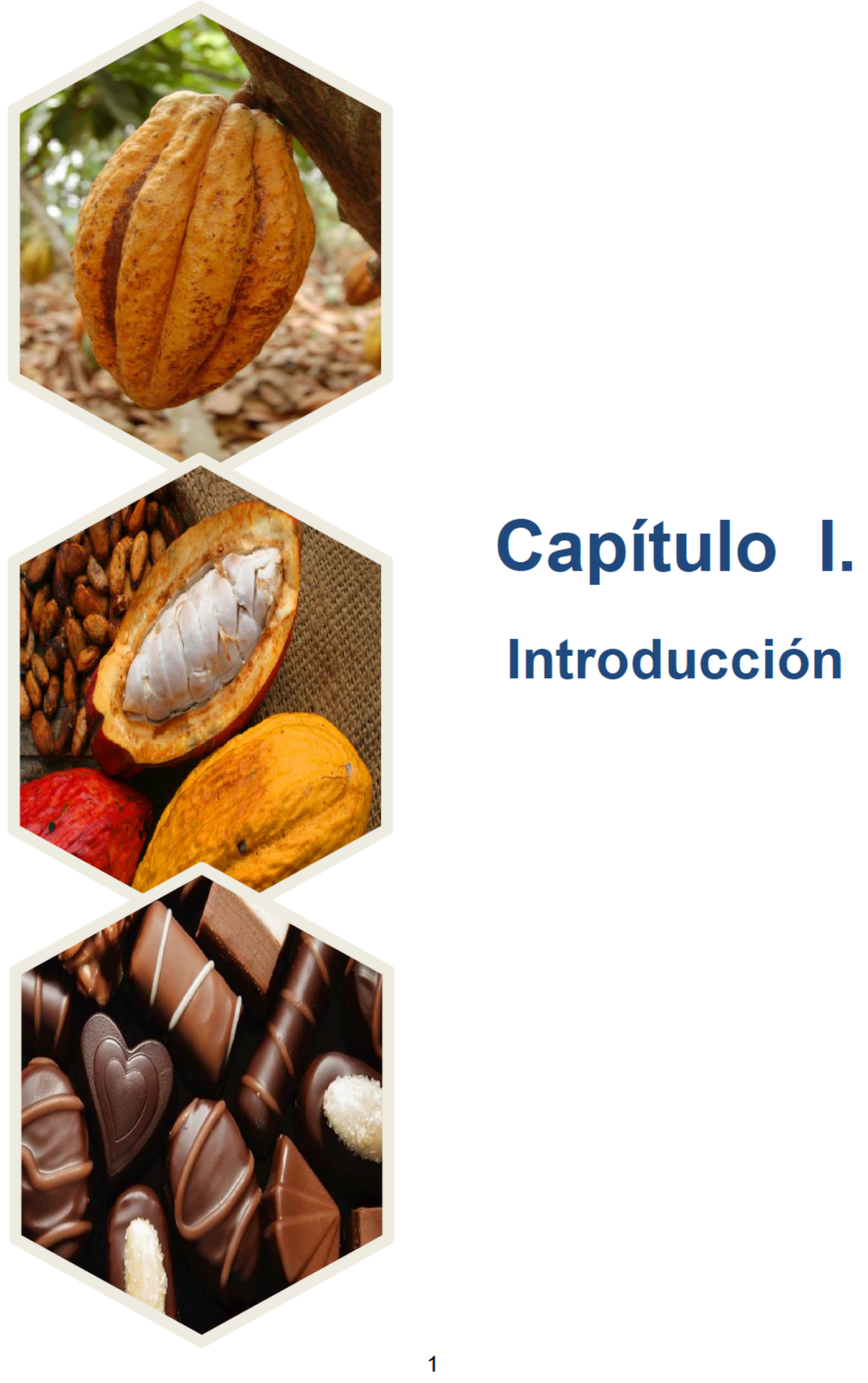




\section{El cacao (Theobroma cacao L.)}

\subsection{Origen}

El cacao (Theobroma cacao L.), es una planta de origen americano, si bien, debido al sistema de vida nómada que siempre llevaron los habitantes en este continente es prácticamente imposible indicar a ciencia cierta cuál fue su lugar de origen (Dand, 2011a; Enríquez, 1985)

Estudios recientes sobre la genética del cacao han demostrado que el centro de origen y diversidad de esta planta se encuentra en la cuenca alta del Amazonas en América del Sur, en países como Perú, Ecuador, Colombia y Brasil; en este último se ha encontrado la mayor variabilidad de la especie. Sin embargo, la población de cacao silvestre también está presente en la cuenca baja del Amazonas, así como a lo largo de la cuenca del río Orinoco en Venezuela y en las Guyanas. Estas poblaciones silvestres fueron distribuidas por humanos en toda América Central y Mesoamérica (Cabrera-Soto, Salinas-Moreno, Velázquez-Cardelas, \& Espinosa-Trujillo, 2009; Enríquez, 1985; Lim, 2012; Loor et al., 2009; Vera Chang J, Cabrera Verdezoto R, Veliz Zamora D, Segovia Freire G, Torres Navarrete Y, Pinargote Alava J, 2018).

La domesticación, cultivo y consumo del Theobroma cacao, se inició en Centroamérica en la época precolombina. Este fue cultivado por primera vez en los años 250-900 dC por las antiguas civilizaciones de los mayas y los aztecas de la región mesoamericana. Los Toltecas, Aztecas y Mayas procesaban estas semillas y producían una bebida llamada "xocoatl". Posteriormente, los europeos incorporaron en su dieta el consumo de cacao en forma acelerada bajo la forma de chocolate, añadiendo azúcar a la mezcla (Batista, 2009; Dand, 2011b).

Después de que el cacao fuera aceptado por los europeos como un alimento necesario, estos comenzaron a llevar semillas de su área natural a otros países. En la actualidad el cacao se cultiva en todos los países que disponen de tierras tropicales y húmedas y se ha convertido en un cultivo verdaderamente popular. Los españoles y los portugueses fueron los promotores de la expansión del cacao, que, habiendo salido de América hoy se encuentra en África, Asia y Oceanía (Batista, 2009; Dand, 2011a; Enríquez, 1985). 


\subsection{Botánica y Agroecología}

El árbol cacaotero (Theobroma cacao L), debe su nombre científico al naturalista sueco Carl Linnaeus debido a que los aztecas creían que el árbol del cacao era de origen divino y que su bebida confería discreción y sabiduría, por lo que le denominó el "alimento de los Dioses", del griego Theobroma. El cacao es una especie altamente alógama (posee mecanismos de fecundación cruzadas), que contiene 20 cromosomas (Batista, 2009; Dand, 2011b; Kim, Lee, \& Lee, 2011; Lim, 2012). En la Tabla 1, se presenta la clasificación taxonómica del cacao.

Tabla 1. Clasificación taxonómica del cacao

\begin{tabular}{ll}
\hline Taxonomía & Clasificación \\
\hline Reino & Plantae \\
Subreino & Tracheobionta \\
División & Magnoliophyta \\
Clase & Magnoliopsida \\
Subclase & Dilleniidae \\
Orden & Malvales \\
Familia & Esterculiacea \\
Subfamilia & Byttnerioideae \\
Tribu & Theobromeae \\
Género & Theobroma \\
Especie & Theobroma cacao L. \\
\hline
\end{tabular}

Fuente: Arévalo et al., 2017

El cacao crece mejor en climas ecuatoriales donde existe abundantes precipitaciones durante todo el año y temperaturas relativamente estables, de entre $25-28^{\circ} \mathrm{C}$. Este árbol se demora de 4 a 5 años para producir frutos y de 8 a 10 años en lograr su máxima producción, esto dependerá de tipo de cacao y las condiciones de la zona (Figura 1) (Jovellanos Fernandez, 2016; Lim, 2012; Mata Anchundia, Suatunces Cuñumai, Munoz Macías, Bayas, \& Herrera Soler, 2018; Muñoz Hernández, 2018; Predan, Lazăr, \& Lungu, 2019)

Sus frutos son leñosos en forma de haba alargada y aparecen sobre la copa de los árboles y debajo de sus ramas. Estos frutos, dependiendo el tipo de cacao pueden ser 
de color amarrillo, blanco, verde o rojo, que oscurecen al madurar. La fruta mide entre 10 y $32 \mathrm{~cm}$ de largo, entre 7 y $10 \mathrm{~cm}$ de ancho y pesa entre $200 \mathrm{~g}$ y $1 \mathrm{~kg}$. En su interior contiene entre 20 y 60 semillas dispuestas en 5 filas rodeadas con una pulpa gelatinosa y azucarada. Cuando el fruto está maduro, se corta y se extraen sus semillas, se las fermenta retirando la baba de la semilla y se seca. El color interno de grano es de color marrón oscuro y tiene un agradable sabor (Lim, 2012; Muñoz Hernández, 2018; Predan et al., 2019; Wilmer Tezara et al., 2016)

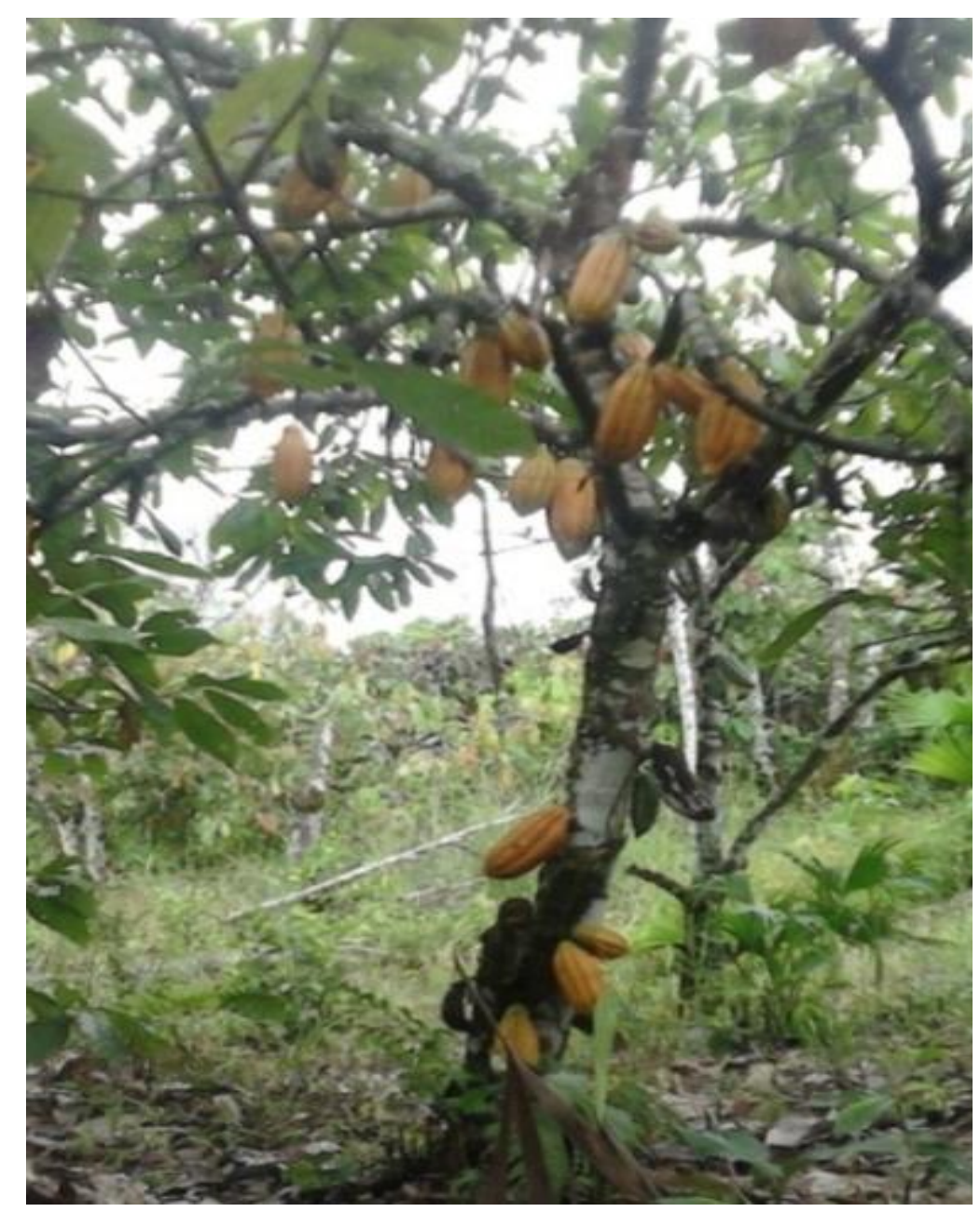

Figura 1. Árbol de Theobroma cacao

\subsection{Cultivares de cacao y perfiles de sabor}

Theobroma cacao L. tiene una diversidad genética significativa y se conocen más de 14000 grupos genéticos distintos de la planta. Los principales grupos genéticos de la especie que se producen comercialmente para la elaboración de chocolate son: Forastero, Criollo, Trinitario y Nacional del Ecuador (Figura 2); estas variedades se distinguen por las características morfológicas de sus frutos, el origen geográfico y las 
características de sabor y aroma (Aprotosoaie et al., 2017; Jovellanos Fernandez, 2016; Loor et al., 2009; Mata Anchundia et al., 2018; Santana, 2017).

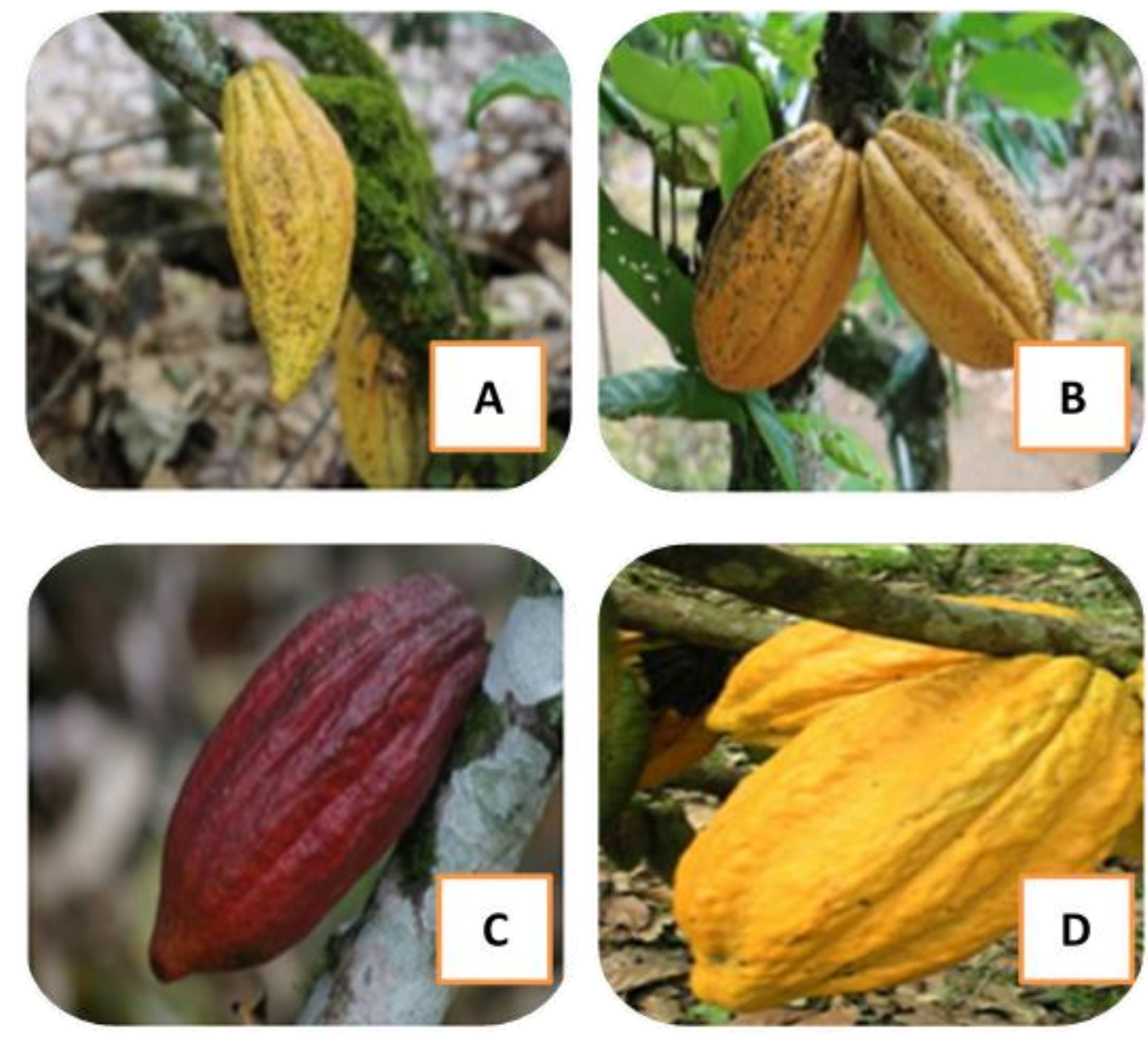

Figura 2. Variedades de cacao a nivel mundial: Criollo (A), Forastero (B), Trinitario(C), Nacional (D)

El cacao del grupo Forastero, se caracteriza por presentar un fruto productivo y vigoroso cultivado desde tiempos históricos. Se produce predominantemente en África occidental y América del Sur, por lo que, representan más del $90 \%$ de la producción mundial. Los granos de cacao Forastero se caracterizan por sus semillas pequeñas, planas, con cotiledones violetas y astringentes debido a la presencia de antocianinas. Estas presentan un fuerte sabor a chocolate y se conocen comúnmente como cacao a granel, básico u ordinario. El cacao Amelonado es la subvariedad más conocida del tipo Forastero, siendo ampliamente cultivada en África Occidental (excepto Camerún) (Aprotosoaie et al., 2017; Balladares Grazzo, 2015; Jovellanos Fernandez, 2016; Konger Edem et al., 2016; Mata Anchundia et al., 2018; Predan et al., 2019; Torres Murillo, 2016; Wood \& Lass, 1985) 
El cacao del grupo Criollo, fue cultivado por los Mayas desde tiempos prehistóricos en América Central y constituye el primer cacao domesticado. Según las pruebas arqueológicas, se encontraron residuos de cacao en recipientes de la cocina Maya que datan de aproximadamente 600 años a.C. A pesar de que estas almendras se han utilizado en alimentos durante más de 2.600 años; actualmente, la producción de este tipo de cacao es muy limitada y sus árboles se encuentran solo en América Central, Venezuela y pocas regiones de Asia (Aprotosoaie et al., 2017; Dand, 2011b; Konger Edem et al., 2016; Predan et al., 2019; Wood \& Lass, 1985). Esta variedad de cacao se caracteriza porque sus frutos maduros son de color amarillo o rojo, las semillas son grandes, redondeadas y con cotiledones de color blanco, además presentan poca resistencia a plagas, cambios climáticos y sus rendimientos de producción son bajos. El cacao criollo es muy aromático y desarrolla sabores suaves, a nuez, flores o (Aprotosoaie et al., 2017; Jahurul et al., 2013; Loor et al., 2009; Predan et al., 2019).

El cacao del grupo Trinitario, se originó en Trinidad y abarca todos los tipos de frutos que se han formado por la hibridación natural entre los grupos Criollo y Forastero. Por lo cual, estos árboles muestran susceptibilidad a plagas y enfermedades intermedias entre las poblaciones los dos grupos mencionados. Las almendras de este cultivar son de color variable, aunque muy raramente de color blanco, presentan características básicas de sabor a chocolate y un sabor a vino que no se encuentran en los otros tipos de cacao (Afoakwa, Paterson, Fowler, \& Ryan, 2008; Loor et al., 2009;Jovellanos Fernandez, 2016; Mundaca Vidarte, 2016; Santana, 2017).

El cacao Nacional se cultiva solo en Ecuador, se caracteriza por tener semillas de color púrpura pálido que producen el famoso sabor "Arriba" que incluye notas aromáticas, florales, especiadas y frutos verdes. Las cultivares que pertenecen a los grupos Criollo, Trinitario y Nacional se clasifican como cacao "fino" y se caracterizan por presentar atributos aromáticos con notas afrutadas, pasas, florales, especiadas, a nueces, melazas y acaramelas. Estos cultivares de cacao se utilizan principalmente para fabricar chocolate negro de alta calidad y representan del 5 al $10 \%$ del mercado mundial (Afoakwa et al., 2008; Aprotosoaie et al., 2017; International Cocoa Organization, 2017; Jahurul et al., 2013; Kongor et al., 2016; Wood \& Lass, 1985).

\subsection{Historia del cultivo de cacao en Ecuador}

La domesticación, cultivo y consumo del cacao fueron iniciados por los indígenas, Toltecas, Aztecas y Mayas en México y Centroamérica mucho antes del 
descubrimiento de América. Estos lo consumían como una bebida llamada xocoatl, que por su sabor amargo no agrado a Moctezuma y su gente. En 1550, Los españoles iniciaron el consumo de esta bebida cuando unas religiosas añadieron dulce y vainilla al chocolate. La bebida que inicialmente era consumida solamente por la corte y realeza europea, pronto pasó a uso más extendido, lo cual origino una gran demanda de la semilla. El cultivo y exportación fueron concedidos mediante Cédula Real como exclusivos en México, Centroamérica, Venezuela y Trinidad y Tobago (Dand, 2011b; Kim et al., 2011; W. Tezara, De Almeida, Valencia, Cortes, \& Bolaños, 2015; Vera Chang J, Cabrera Verdezoto R, Veliz Zamora D, Segovia Freire G, Torres Navarrete Y, Pinargote Alava J, 2018).

A pesar de que Ecuador tenía la exclusividad de obrajes y lanas, en la segunda mitad del siglo XVI fue tan rentable el negocio del cacao, que atrajo el interés de los empresarios de la ciudad de Guayaquil en la costa del Pacífico ecuatoriano, quienes empezaron a cultivar este producto, a pesar de las prohibiciones establecidas mediante las Cedulas Reales. En 1623, el corregidor de Guayaquil, don Diego de Portugal, informa a la Corte de España que había un gran número de plantas sembradas en la provincia y que su producto era comercializado clandestinamente desde Guayaquil, primero por Acapulco y posteriormente por prohibiciones desde España, salía por puertos de Sonsonate (Nicaragua), Ajaculta y Amapola (Guatemala) (Jimenez, J., Amores, F., \& Solórzano, Jiménez, Amores, \& Solórzano, 2014).

La producción y comercio clandestino desde Guayaquil en lugar de detenerse, siguió en aumento, pero esta vez con envíos a Acapulco desde el Callao, lo cual motivo que el Cabildo de Caracas (1593 a 1778) elevara quejas y solicitudes al Rey y las cortes para frenar la producción y el negocio de cacao en Ecuador, pero sin tener éxito. Finalmente, en 1789, el Rey Carlos IV permitió mediante Cedula Real, el cultivo y exportación de cacao desde la costa ecuatoriana (Enríquez, 1985).

Según fuentes históricas, desde el principio de 1600 ya había pequeñas plantaciones de cacao a orillas del Rio Guayas y se expandieron a orillas de sus afluentes el Daule y el Babahoyo, rio arriba; lo cual origino el nombre de cacao "Arriba" en el mercado internacional, que va ligado a su denominación de origen. Las variedades que dan origen a este cacao se denominan "Nacional" y botánicamente pertenece a las poblaciones de forasteros amazónicos. Los árboles de cacao Nacional desarrollan un fuerte aroma floral conocido como sabor "arriba" en los mercados internacionales, que 
lo demandan para hacer productos de chocolatería específicos y se produce exclusivamente en Ecuador (Mata Anchundia et al., 2018; Muñoz Hernández, 2018; Vera Chang J, Cabrera Verdezoto R, Veliz Zamora D, Segovia Freire G, Torres Navarrete Y, Pinargote Alava J, 2018; Zambrano Tenezaca, 2017)

Durante los años de lucha por la independencia del Ecuador (1800 - 1822), la producción de cacao fue su mayor fuente de financiamiento, en esa época representó entre el 40 al $60 \%$ de las exportaciones totales del país y llegó a pagar hasta el $68 \%$ de los impuestos del Estado. Las nuevas leyes de la República sobre concesiones de tierras, permitieron que muchas familias con recursos económicos de la costa ecuatoriana adquirieran grandes propiedades y las destinaran al cultivo de cacao. Las áreas preferidas fueron las llamadas "Arriba" en la actual provincia de Los Ríos (Vinces, Babahoyo, Palenque, Baba, Pueblo Viejo, Catarama y Ventanas), al sur de la provincia del Guayas (Naranjal, Balao, Tenguel) y en El Oro (Machala y Santa Rosa) (Asociación Nacional de Exportadores de Cacao, 2015; Dand, 2011b; Enríquez, 1985).

El auge cacaotero se vio drásticamente afectado con la llegada de enfermedades como la Escoba de Bruja y la Monilla entre los años 1915 y 1920. Desde su aparición estas se expandieron en toda la zona cacaotera causando una reducción de la producción de 40000 a 15000 TM, para el año 1930. A este desastre económico, social y técnico se sumó los efectos de la Primera Guerra Mundial, que provoco la falta de transporte y el cierre de mercados internacionales, con una consecuente depresión económica del país en esos años (Asociación Nacional de Exportadores de Cacao, 2015; Dand, 2011b).

Está crisis promovió la venta de la mayoría de las grandes haciendas, que fueron compradas por pequeños y medianos propietarios, quienes comenzaron desde fines de la década de los años 30 y 40, a renovar y sembrar nuevas huertas. Los pequeños agricultores utilizaron semillas de los árboles que habían tolerado las enfermedades, dando origen al complejo varietal, actualmente denominado hibrido Nacional $x$ Venezolano. Este hibrido es el resultado del cruzamiento natural entre los arboles sobrevivientes de la variedad Nacional y árboles de la variedad Trinitario, introducida de Venezuela a principios de siglo por considerarlas más productiva y tolerantes a las enfermedades (Asociación Nacional de Exportadores de Cacao, 2015). 
En la década de 1960 se produce un nuevo incremento de áreas de siembra con la llegada de la Reforma Agraria que en base a repartición de tierras baldías y de haciendas improductivas consiguió elevar la producción hasta 33000 TM. Para 1980 los niveles de producción siguieron en crecimiento alcanzando un promedio de 80000 TM por año. Actualmente, el área aproximada de cultivo alcanza las 360000 ha, en todas las zonas tropicales de Ecuador encontrándose cultivos en la región Costa, Amazonía y parte de la Sierra (Asociación Nacional de Exportadores de Cacao, 2015; Enríquez, 1985).

\subsection{Importancia socioeconómica del cacao en Ecuador}

El cacao es conocido en el Ecuador como la "pepa de oro", su denominación se debe a que este rubro dominó por varios siglos la generación de divisas para el país, antes del boom petróleo. Este cultivo dio lugar al aparecimiento de los primeros capitales y desarrolló sectores importantes como la banca, la industria y el comercio. Durante casi un siglo, el orden socioeconómico ecuatoriano se desarrolló en gran medida alrededor del mercado internacional del cacao (PROECUADOR, 2013).

Actualmente el cacao juega un papel importante en la transformación de la matriz productiva del país, y representa una preocupación creciente en todos los ámbitos de la sociedad y el Gobierno, generando políticas tendenciales a tal fin (Carrasco, 2015).

Su producción está localizada en 20 de las 24 provincias y se produce como monocultivo o asociado a otras especies. La mayor concentración del cultivo de cacao se encuentra en las provincias del Litoral (Los Ríos, Guayas, Manabí, Esmeraldas y El Oro), en la región Sierra este frutal se desarrolla en las estribaciones del Cordillera Occidental de los Andes (Bolívar, Chimborazo, Cañar y Azuay) y en las provincias del norte de la región Amazónica del Ecuador (Sucumbíos, Orellana y Napo) (CEPAL, 2013). Los datos disponibles según la Encuesta de Superficie y Producción Agropecuaria Continua (ESPAC), realizada en el año 2019, a nivel nacional se registra 601954 ha de superficie sembrada, 525435 ha de superficie cosechada. La producción actual alcanza las $283680 \mathrm{t}$ con un rendimiento promedio de $0,54 \mathrm{t} \cdot \mathrm{ha}^{-1}$, por sus bajas tasas de rendimiento el Ecuador se ubica en la séptima posición entre los países productores. Esto se debe a la falta de tecnología, elevado número de plantaciones viejas y la baja resistencia del cacao Nacional a plagas (Carrasco, 2015; García-Vidal, Guzmán-Vilar, \& Campdesuñer, 2017; López-Guerrero, 2017). 
Este cultivo generó empleo para cerca de 100000 familias de productores ecuatorianos en el año 2017; de los cuales se estimó que el 80\% cultivó menos de 10 ha, un 15\% tenía huertos que van de 10 a 20 ha, y un 5\% tenía más de 20 ha. En este año, además de los agricultores, participaron en la cadena del cacao otras 20000 familias, lo que representó una influencia directa sobre 600000 personas (LópezGuerrero, 2017).

En el año 2017, dentro de las exportaciones no petroleras tradicionales; la cadena de valor del cacao fue la tercera más relevante después del banano, plátano y camarón. La producción en ese año fue de 308000 toneladas, con ingresos por concepto de ventas de alrededor de 689 millones de dólares (García-Vidal et al., 2017). Para el primer semestre del año 2018, las exportaciones de cacao ecuatoriano estuvieron destinadas principalmente a Estados Unidos, Indonesia, Malasia y Holanda (EL Telégrafo, 2019; García-Vidal et al., 2017; López-Guerrero, 2017)

\subsection{Tratamiento poscosecha del cacao}

El tratamiento poscosecha del cacao incluye todo el proceso primario desde la cosecha de las mazorcas hasta obtener los granos secos para la comercialización. Estos procesos se realizan en la zona de producción del cacao y juegan un rol fundamental en el desarrollo del perfil aromático de los granos secos (Kongor et al., 2016). En el proceso poscosecha se clasifican las mazorcas para separar los frutos enfermos y aquellos que no tengan un grado de madurez adecuado. Posteriormente, se realiza la apertura de las mazorcas y la extracción de las almendras que conformarán la masa para la fermentación (Kongor et al., 2016; Páramo et al., 2010).

\subsubsection{Fermentación}

La fermentación es un proceso microbiológico y bioquímico que conduce a la eliminación de la pulpa o mucílago, provoca la muerte del embrión, activa las reacciones bioquímicas en el interior de los cotiledones e intensifica el desarrollo de los precursores del sabor y aroma (Hartel, von Elbe, \& Hofberger, 2018; Kongor et al., 2016; Pinto-Mosquera, 2011).

Gran parte del proceso de fermentación ocurre en condiciones anaeróbicas, aunque la mezcla o remociones de las almendras que se realiza periódicamente durante este proceso, le agrega oxígeno y permite que también ocurra la fermentación aeróbica. La 
fermentación debe controlarse cuidadosamente ya que los tiempos de fermentación muy largos produce almendras sobre fermentadas; que se caracterizan por presentar una acidez excesiva y sabores indeseables (sabores a humedad o a basura). Los cambios químicos que ocurren durante la fermentación son bastante complejos y aún no se aclaran por completo a pesar de años de estudio. Numerosas especies microbianas, incluidos hongos, levaduras, bacterias ácido lácticas y bacterias ácido acéticas, participan en la fermentación (Afoakwa, Quao, Simpson Budu, Takrama, \& Kwesi Saalia, 2011; CAOBISCO/ECA/FCC, 2015; Kongor et al., 2016). La fermentación va acompañada de numerosas reacciones enzimáticas por acción de proteasas, peptidasas, invertasa, polifenol oxidasa y glucosidasas, todas contribuyendo a la generación de moléculas precursoras de sabor y aroma (Aprotosoaie, Luca, \& Miron, 2016; Gibson \& Newsham, 2018; Hartel et al., 2018).

Por las características fisicoquímicas del mucilago $(\mathrm{pH}: 3.4$ a 4.0; glucosa 12-15\% y ácido cítrico $2 \%$; pectinas $5-7 \%$ ) y la baja tensión de oxígeno, el proceso fermentativo inicia con la proliferación de levaduras como Saccharomyces cerevisae, Candida Krusei, Kloeckera apiculata, mismas que dominan la fermentación durante las primeras 24 a 36 h; dependiendo de la variedad de cacao (Ooi, Ting, \& Siow, 2020; Romanens et al., 2019; Santos et al., 2020).

En la primera etapa las levaduras consumen los azúcares de la pulpa para transformarlos en etanol hasta alcanzar una concentración máxima del 5 a $6 \%$ provocando cambios de $\mathrm{pH}$ y de la estructura de la pulpa, mismos que permitirán el desarrollo de bacterias lácticas (Leuconostoc, Lactobacillus, Streptococcus). Después de la primera remoción la presencia de oxigeno favorece el crecimiento de bacterias acéticas (Acetobacter rancens, A. ascendes, A. xylinum, A. lovaniensis, A. aceti y Gluconobacter oxydans) que junto con las bacterias lácticas disminuyen el contenido de etanol (1\%) produciendo ácidos orgánicos como málico, láctico y acético, en ese orden; para finalmente producir $\mathrm{CO}_{2}$ y agua. En esta fase el ácido cítrico de la pulpa es consumido y el $\mathrm{pH}(4,5)$ y la temperatura $\left(40^{\circ} \mathrm{C}\right)$ aumentan dando lugar a numerosos compuestos volátiles (Hartel et al., 2018; Ooi, Ting, Siow, et al., 2020; Owusu, Petersen, \& Heimdal, 2011; Petersen, Blennow, \& Nielsen, 2014; Santos et al., 2020; Thuy, Zhao, \& Fleet, 2015).

La fermentación acética es un punto clave del proceso, puesto que el ácido acético provoca una baja del $\mathrm{pH}$ que junto con el calor de la fermentación $\left(50^{\circ} \mathrm{C}\right)$ causan la 
muerte celular de las almendras durante el segundo día de fermentación. La muerte de las almendras está acompañada de un aumento en la permeabilidad de las paredes celulares, lo cual permite la interdifusión de los componentes del jugo celular ricos en enzimas cuyo sustrato son polifenoles y proteínas (Hartel et al., 2018; Kongor et al., 2016; Sarbu \& Csutak, 2019).

Tan pronto se muere la semilla, la enzima glicosidasa se activa hidrolizando los compuestos responsables del color púrpura de las almendras conocidos como antocianinas (3- $\beta$-D-galactosidil cianidina y la 3- $\alpha$ - L- arabinosidil cianidina). En esta reacción se produce la decoloración gradual de las almendras por descomposición de los azúcares y antocianidinas. Éstas últimas luego se someterán a reacciones de condensación oxidativa formando polímeros de alto peso molecular como los taninos que contribuyen a la formación de color marrón característico del cacao fermentado (Afoakwa et al., 2011; Hartel et al., 2018; Lefeber, Papalexandratou, Gobert, Camu, \& Vuyst, 2012).

Las proteínas son hidrolizadas por dos proteasas específicas que conducen a la formación de más de 80 oligopéptidos y aminoácidos libres diferentes. La especificidad de las enzimas en estas reacciones depende del $\mathrm{pH}$. Las proteínas son hidrolizadas por una endoproteasa aspártica ( $\mathrm{pH}$ óptimo 3,5) que conduce a la formación de oligopéptidos. La hidrólisis de estos sustratos por una carboxipeptidasa ( $\mathrm{pH}$ óptimo 5.5-5.8) conduce a la formación de oligopéptidos hidrófilos y de aminoácidos libres hidrófobos (Afoakwa et al., 2011; Aprotosoaie et al., 2017; CAOBISCO/ECA/CFC, 2015; Hartel et al., 2018).

Posteriormente, las procianidinas y las proteínas o los productos de su degradación se combinan durante esta etapa. Ninguna de estas reacciones incluye oxígeno. Durante la fase de fermentación debe mantenerse una aireación que no sea muy escasa, porque impediría la multiplicación de las levaduras y bacterias acéticas, que estaría acompañada de una reducción de ácido acético y del calor generado, ni tampoco excesiva, por cuanto en este caso no solamente daría lugar al desarrollo de mohos, sino que también impediría la formación del precursor de sabor (Afoakwa et al., 2008; Enríquez, 1985; Hartel et al., 2018).

A medida que el oxígeno se difunde dentro de los tejidos, la oxidasa se activa y las condiciones aeróbicas, así como los productos de oxidación de los polifenoles, 
progresivamente inhiben las enzimas que estaban activas durante la fase anaeróbica. Las antocianidinas y los complejos fenolproteína que se formaron durante la fase anaeróbica sufren reacciones oxidativas. Los antocianos son polimerizados junto con catequinas presentes en el cacao, por la enzima polifenoloxidasa formando taninos complejos insolubles, pero esta disminución en el contenido de polifenoles no es debido solo a los procesos de oxidación sino también es provocado por el pardeamiento y la difusión de estos componentes en los exudados de la fermentación (Hartel et al., 2018; Wollgast \& Anklam, 2000).

En resumen, los factores que influyen en la fermentación de los granos de cacao incluyen; la naturaleza de la microflora local, las características de la pulpa $(\mathrm{pH}$, contenido de agua, cantidad, composición, etc.) y las condiciones de fermentación (temperatura, tiempo, frecuencia de remociones para comenzar el proceso aeróbico etc.). En forma general, desde el punto de vista sensorial y bioquímico durante la fermentación se producirá un incremento de los contenidos en azúcares reductores y aminoácidos libres hidrófobos, que son los precursores del aroma, y una disminución de las purinas y compuestos fenólicos que ayudan a que el cacao sea menos amargo y astringente (Afoakwa et al., 2008; de Souza, Moreira, Sarmento, \& da Costa, 2018; Hartel et al., 2018; Sarbu \& Csutak, 2019; Wollgast \& Anklam, 2000).

\subsubsection{Métodos de fermentación}

El sistema de fermentación del cacao varía en función del volumen de producción de la finca, siendo complejo y costoso cuando los volúmenes cosechados son grandes. Este proceso dura generalmente entre 3 y 6 días y puede realizarse de diferentes formas, las mismas que pueden ser el resultado del desarrollo de tecnologías de centros de investigación o por experiencia técnica-investigativa empírica del agricultor.

Los métodos más comunes utilizados en Ecuador son: cajas de madera, sacos de cabuya, montones, y gavetas Rohan, todas tienen como principio crear un ambiente semi-cerrado, que permita eliminar los líquidos de exudación de las almendras y que estas alcancen la temperatura de $45^{\circ} \mathrm{C}$, para así matar al embrión e iniciar el proceso químico de transformación (Balladares Grazzo, 2015; Guanga, 2018; JovellanosFernández, 2016; Pinto-Mosquera, 2011). 


\section{a) Cajas de Madera}

Las cajas de maderas blancas y sin sustancias como taninos o resinas permite obtener los mejores resultados durante la fermentación, siendo el laurel el mejor material (Figura 3). Este método consiste en colocar las almendras frescas en cajas de madera fuerte con fondo perforado para el drenaje de las exudaciones.

Después de permanecer en una caja por uno o dos días, las almendras se trasladan a una segunda caja; durante esta operación que se efectúa con palas de madera, hay un escape de anhídrido carbónico y la temperatura desciende apreciablemente. La temperatura vuelve a subir pronto y prosigue la cura. Después de dos o tres días más, las almendras se ponen en una tercera caja y se mantienen allí hasta completar unos 6 días, generalmente no más de 8 (Enríquez, 1985; Páramo et al., 2010; PintoMosquera, 2011).

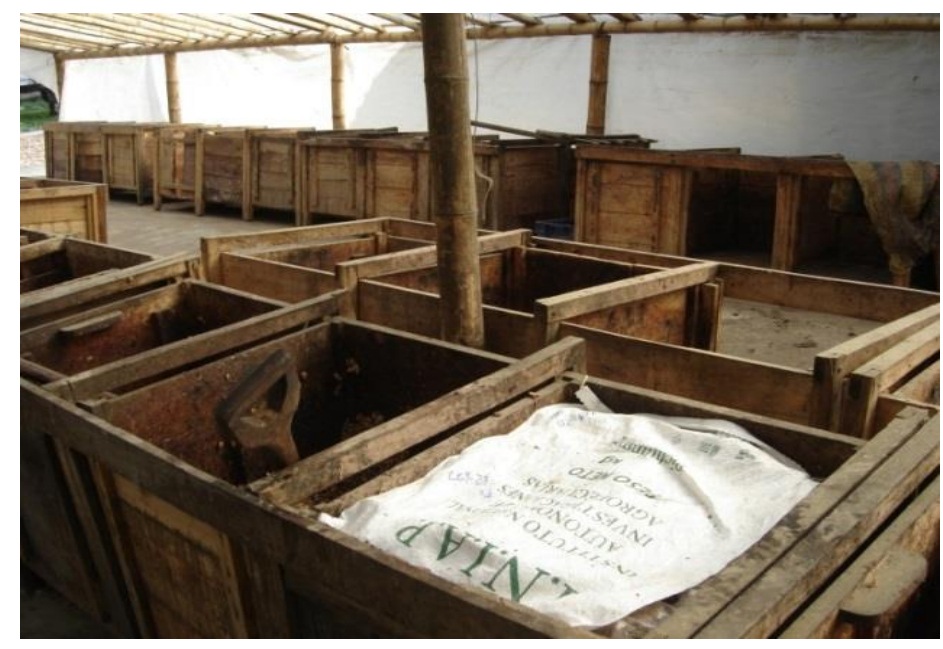

Figura 3. Fermentación de cacao en cajones de madera

\section{b) Montones}

Es quizás el método mayormente utilizado por los pequeños y medianos agricultores (Figura 4); consiste en colocar cacao en baba sobre un tendal de caña guadua picada o de madera, preferentemente elevado al menos $15 \mathrm{~cm}$ del suelo para proteger las almendras de encharcamientos causados por los líquidos o excesos de humedad. La masa en fermentación se debe cubrir con hojas de plátano para conservar la temperatura y evitar contaminación por hongos. Esos montones deben removerse de 
un lugar a otro, para obtener una mejor fermentación (Dand, 2011a; Páramo et al., 2010; Pinto-Mosquera, 2011).

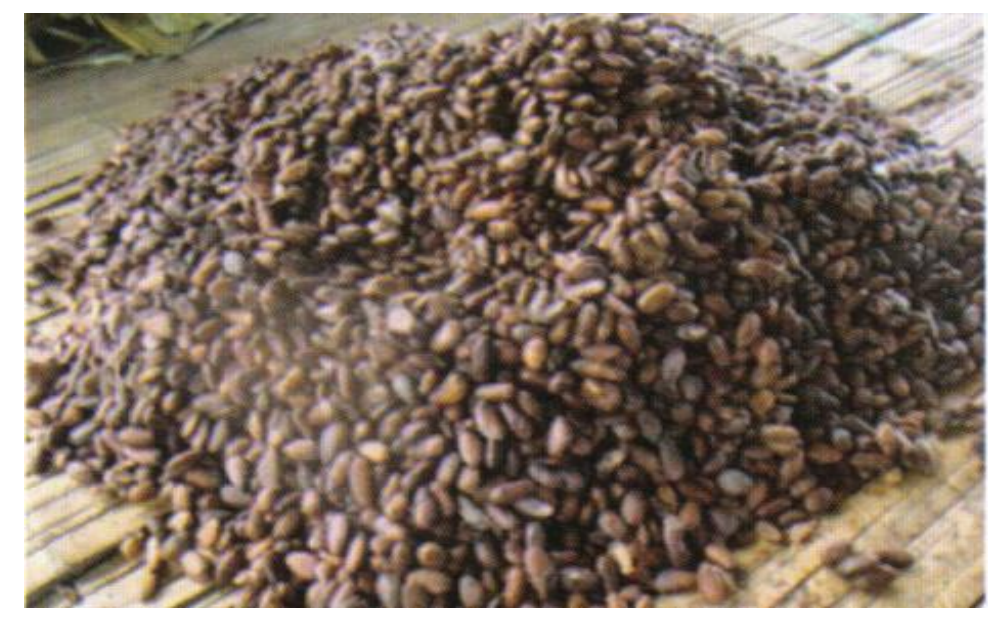

Figura 4. Fermentación de cacao en montones

\section{c) Sacos}

En este método, los granos frescos de cacao se colocan en sacos (Figura 5), preferentemente nuevos o exclusivos para cacao y de tejido ralo para permitir el exudado de la baba. En la fermentación por saquillos generalmente las personas no realizan remociones de la masa motivo por el cual el grado de fermentación no es uniforme. Además, cuando los saquillos plásticos tienen un tejido muy tupido, el cacao tiende a quedar con cierto sabor a podrido. Por lo tanto, este es el método menos adecuado para fermentar cacao, debido al bajo porcentaje de fermentación y calidad inferior obtenida (Dand, 2011a; Pinto-Mosquera, 2011).

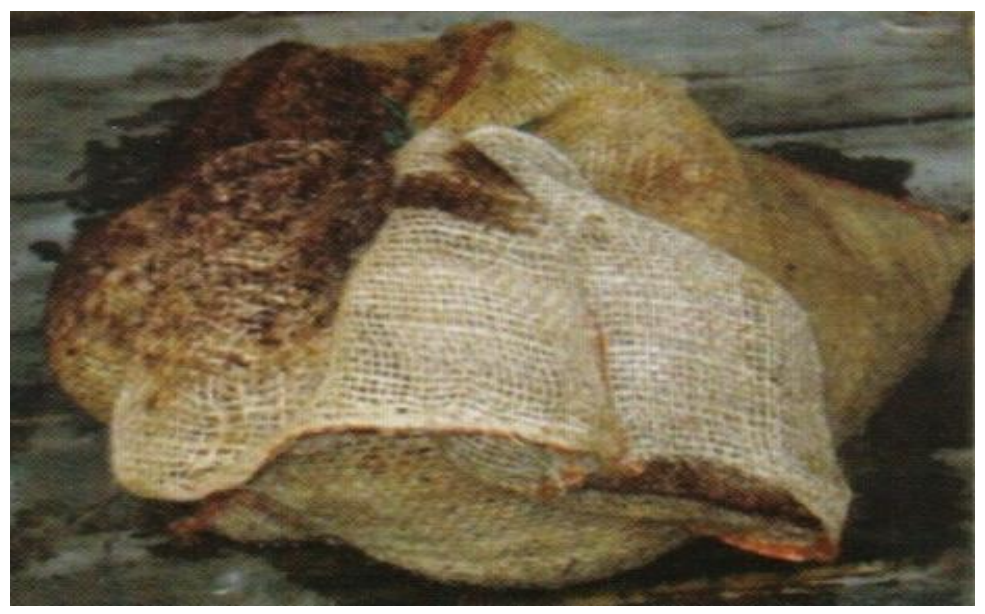

Figura 5. Fermentación de cacao en sacos 


\section{d) Fermentación en Gavetas Rohan}

Este procedimiento de fermentación consiste en colocar las almendras a fermentar, en gavetas de 1,20 m de largo $\times 0,80 \mathrm{~m}$ de ancho $\times 0,10 \mathrm{~m}$ de alto, y pueden ser apiladas hasta un total de 12 a 16 gavetas. Estas son tapadas en la parte superior con tela de gangocha o lonas útiles para el proceso. El piso de las gavetas deberá tener aberturas de entre 5 a $10 \mathrm{~cm}$ lo cual permite una aireación de las almendras, de esta forma se puede presentar una fermentación más general en las almendras sin la necesidad de mover las mismas (Figura 6). En algunos lugares del mundo las Gavetas Rohan son fabricadas de bambú, ya que este es el material principal de la región, los materiales de este método variaran de acuerdo al lugar en cuestión (Dand, 2011a; Jiménez et al., 2011).

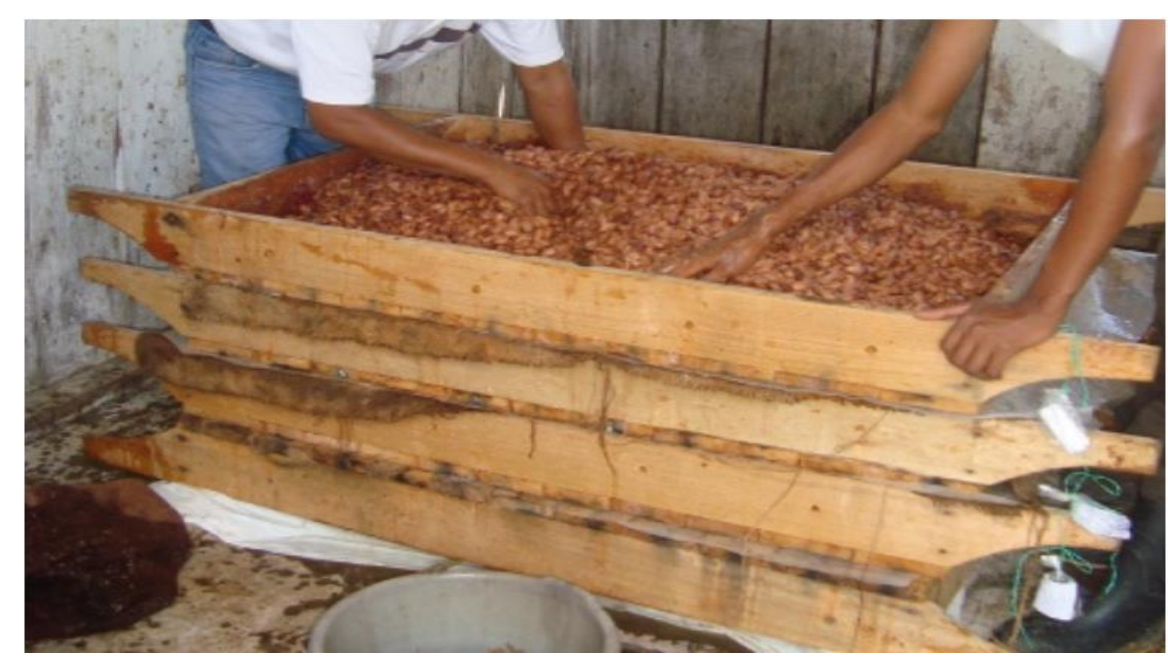

Figura 6. Fermentación en gavetas Rohan

\subsubsection{Secado}

Al culminar el proceso de fermentación, las almendras de cacao tienen alrededor del $55 \%$ de humedad. Por el alto riesgo microbiológico que representa las almendras recién fermentadas, deben ser sometidas a un proceso de secado para eliminar el exceso de agua de su interior hasta alcanzar 7-8\% de humedad. Esto evita la putrefacción por el desarrollo de mohos que deterioren la calidad, dando como resultado facilidad en las condiciones de almacenamiento, manejo y comercialización del cacao (Aprotosoaie et al., 2016; Dand, 2011a; Hartel et al., 2018; Mondino \& Ferrato, 2006). 
En la etapa de secado continúa la fase oxidativa iniciada en la fermentación terminándose algunas transformaciones químicas y físicas, contribuyendo a la generación de características organolépticas (compuestos del aroma y sabor) y el desarrollo de pigmentos de color marrón a partir de los compuestos fenólicos (Aprotosoaie et al., 2016; Hartel et al., 2018).

Los procesos de secado se realizan de forma natural (calor del sol) o en secadores artificiales. Los más comunes en Ecuador son el secado natural al sol en tendales con superficie de secado de madera, caña guadua o cemento (Figura 7A) y el secado en marquesinas (pisos de madera) con estructuras cubiertas de plástico que dejan pasar la luz del sol y protegen las almendras de las lluvias impredecibles (Figura 7B). Los métodos de secado artificial utilizan algunos tipos de combustibles para generar calor y acelerar el secado, también existen secadores eléctricos, cuya energía es más barata que la de los combustibles (Dand, 2011a; Enríquez, 1985).

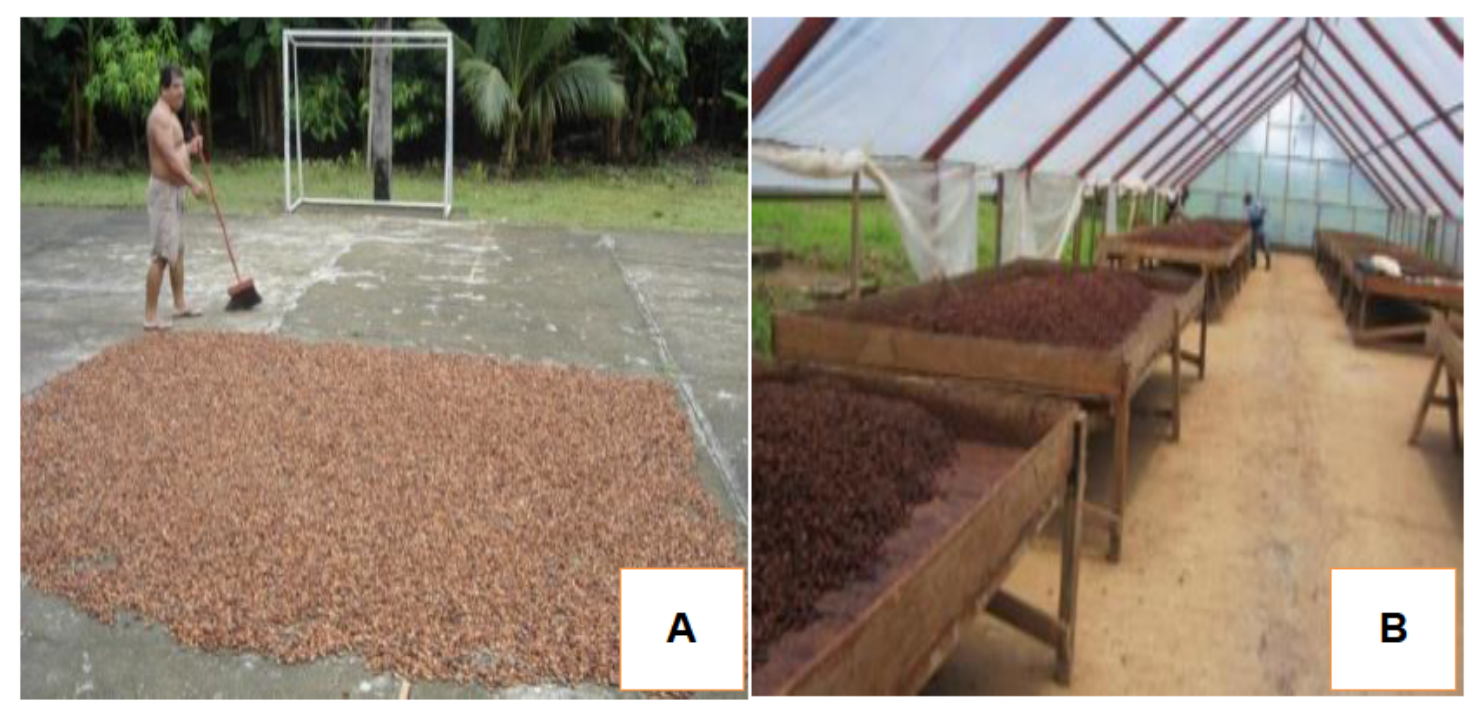

Figura 7. Secado natural de cacao sobre tendales de cemento (A) y en marquesinas (B)

Durante este proceso de secado se pone en evidencia la aparición de nuevos compuestos y la desaparición de otros inicialmente presentes. Estos nuevos compuestos pueden ser de origen bioquímico (ésteres, aldehídos y cetonas) o térmico (aldehídos, pirazinas, pirroles y fenoles). Entre los componentes que más disminuyen están los alcoholes y los ácidos. Así mismo, las familias de los aldehídos, cetonas, ésteres, pirazinas, pirroles y compuestos azufrados aumentan durante el secado. Los fenoles disminuyen a partir del tercero y cuarto día. Con respecto al resto de los compuestos de cada una de las familias, está claramente estudiado que muchos de 
ellos tienen importancia en las características aromáticas del cacao y por ende se relacionan con la calidad sensorial del chocolate (Afoakwa et al., 2008; Aprotosoaie et al., 2016; Hartel et al., 2018; Predan et al., 2019; Zambrano, 2018).

\subsection{Atributos de calidad y composición química del cacao}

Las almendras de cacao constituyen la principal materia prima para la elaboración de chocolates y productos derivados, pueden ser incorporadas en otros productos que responden a las más exigentes demandas de la industria agroalimentaria (confitería, pastelería, bebidas instantáneas, productos lecheros etc.), farmacéutica y cosmética. El cacao es muy apetecible en el mercado mundial, debido a que posee un aroma y sabor inimitable. Además, de su rica composición en compuestos bioactivos y sus características de calidad (CAOBISCO/ECA/CFC, 2015; Hartel et al., 2018; Pontillon, 1997).

Comercialmente en el mercado mundial se reconoce dos tipos de cacao en grano: el "fino o de aroma" y el "común o al granel". Generalmente el cacao fino y de aroma es producido por árboles de cacao de variedad Criollo o Trinitario, mientras que, el cacao al granel proviene de los árboles de cacao Forastero. El 95\% de la producción mundial de cacao es a granel, este tipo de cacao se produce en su mayor parte en África, Asia y Brasil. El $5 \%$ de la producción restante corresponde a cacao fino de aroma, siendo los mayores productores Ecuador (primer productor), Indonesia, Papua Nueva Guinea, Colombia, Venezuela y Trinidad (Afoakwa et al., 2008, 2011; Dand, 2011b; Konger Edem et al., 2016).

La calidad de los granos de cacao depende de varios factores como el genotipo, el manejo agronómico, las condiciones del suelo, las condiciones climáticas y el factor más importante el manejo poscosecha (fermentación y secado de los granos). Esta calidad se manifiesta a través de parámetros físicos, organolépticos y químicos. Los parámetros químicos son atributos propios del tipo de cacao o se desarrollan durante el proceso de fermentación, secado y torrefacción. Dichas características químicas proporcionan información cuantitativa para caracterizar diferentes tipos de cacao y permiten evaluar el proceso de beneficio poscosecha del cacao. Por lo tanto, la calidad del cacao debe interpretarse a partir de la identificación de diferentes atributos químicos, sus relaciones entre sí y con el medio ambiente (Acierno, Yener, Alewijn, Biasioli, \& van Ruth, 2016; Adeyeye \& Akinyeye, 2017; Afoakwa et al., 2008, 2011; 
Brunetto et al., 2007; Espín \& Samaniego, 2016; Hue et al., 2014; Konger Edem et al., 2016).

En la actualidad, la calidad del cacao Nacional ecuatoriano beneficiado se evalúa mediante la norma técnica NTE INEN 176:2006, "Granos de cacao Requisitos" (Tabla 2), la misma que utiliza parámetros físicos como: peso de 100 granos, porcentaje de cáscara, porcentaje de humedad, nivel de fermentación entre otros; además se utilizan pruebas organolépticas, las mismas que son subjetivas. Debido a la importancia comercial y económica del cacao, desde hace algunos años se ha venido trabajando en el desarrollo de pruebas objetivas basadas en la composición química de las almendras que permita certificar su origen y mejorar los precios en el mercado internacional (Araujo et al., 2014; Instituto Nacional Ecuatoriano de Normalización, 2018; Jimenez, J., Amores, F., \& Solórzano et al., 2014; Loureiro et al., 2017).

Tabla 2. Requisitos de calidad del cacao beneficiado

\begin{tabular}{|c|c|c|c|c|c|c|c|}
\hline & UNIDAD & A.S.S.P.S & A.S.S.S & A.S.S & A.S.N & ASE & \\
\hline Cien gramos pesan & $g$ & $135-140$ & $130-135$ & $120-125$ & $110-115$ & $105-110$ & $135-140$ \\
\hline Buena fermentación (min) & $\%$ & 75 & 65 & 60 & 44 & 26 & $65^{\star * *}$ \\
\hline Ligera fermentación (min) & $\%$ & 10 & 10 & 5 & 10 & 27 & 11 \\
\hline Violeta (máx.) & $\%$ & 10 & 15 & 21 & 25 & 25 & 18 \\
\hline Pizarroso (pastoso) máx. & $\%$ & 4 & 9 & 12 & 18 & 18 & 5 \\
\hline Moho (máx.) & $\%$ & 1 & 1 & 2 & 3 & 4 & 1 \\
\hline $\begin{array}{l}\text { Total (análisis sobre } 100 \\
\text { pepas) }\end{array}$ & $\%$ & 100 & 100 & 100 & 100 & 100 & 100 \\
\hline $\begin{array}{l}\text { Defectuosos (máximo análisis } \\
\text { sobre } 500 \text { gramos) }\end{array}$ & $\%$ & 0 & 0 & 1 & 3 & $4^{* *}$ & 1 \\
\hline Total fermentada (min.) & $\%$ & 85 & 75 & 65 & 54 & 53 & 78 \\
\hline
\end{tabular}

Arriba Superior Summer Plantación selecta (A.S.S.P.S); Arr ba Superior Summer Selecto (A.S.S.S); Arriba Superior Selecto (A.S.S.); Arriba Superior Navidad (A.S.N.); Arriba superior Época (A.S.E.).

Coloración marrón violeta $\left(^{*}\right)$; se permite la presencia de granza solamente para el tipo A.S.E. $\left(^{* \star}\right)$; la coloración varía de marrón a violeta $\left({ }^{* * *}\right)$

Fuente: Instituto Nacional Ecuatoriano de Normalización (2018)

En este contexto, se ha propuesto diferentes índices de calidad del cacao para clasificar muestras de diferentes orígenes geográficos, en función de la evaluación 
simultánea de los atributos bioquímicos del grano. Estos índices se han mejorado a partir de la información actualizada y tomando como base diferentes atributos y métodos analíticos en varias regiones productoras. Por lo tanto, los datos bioquímicos sobre la calidad del cacao son el paso inicial hacia el desarrollo de estudios más sistemáticos y exhaustivos (Araujo et al., 2014; Loureiro et al., 2017).

Las almendras de cacao fermentadas y secas están constituidas por metabolitos primarios como: carbohidratos, lípidos y proteínas, así como metabolitos secundarios como: compuestos polifenólicos, bases nitrogenadas como alcaloides (metilxantinas), estos compuestos influyen sobre el sabor y aroma del chocolate. Los principales constituyentes bioquímicos del cacao son: lípidos (48-57\%), compuestos polifenólicos (8-20\%), proteínas (9\%) y carbohidratos (celulosa 9\%; almidón 4.5 - 7\% y pectina $4.1 \%$ ), estas fracciones representan más del $95 \%$ de la composición de grano (Tabla 3) (Aprotosoaie et al., 2016; de Souza et al., 2018; Loureiro et al., 2017).

Tabla 3. Composición química nutricional del grano de cacao beneficiado

\begin{tabular}{lc}
\hline \multicolumn{1}{c}{ Compuesto } & Contenido (\%) \\
\hline Grasa & $48-57$ \\
Compuestos fenólicos & $8-20$ \\
Proteína & 9 \\
Celulosa & 9 \\
Almidón & $4.5-7$ \\
Pectina & 4.1 \\
Cenizas & $2.6-4.2$ \\
Teobromina & $1.3-2$ \\
Aminoácidos libres & 0,8 \\
Cafeína & $0.1-0-7$ \\
Glucosa & 0,1 \\
Fructosa & $0.4-0.6$ \\
Ácidos orgánicos & $0.05-0.5^{*}$ \\
\hline *Por cada ácido orgánico (málico, láctico, cítrico) \\
Fuente: de Souza et al. ( 2018)
\end{tabular}

Este grupo de compuestos químicos no volátiles presentes en los granos fermentados y secos, participan en la formación de sabores específicos en el cacao, por lo cual, se conocen como precursores de sabor y aroma (metilxantinas, polifenoles, proteínas y carbohidratos). El genotipo del cacao, las condiciones de cultivo y el medio ambiente son los principales factores que determinan la variabilidad de los contenidos de estos 
componentes en los granos (Acierno et al., 2016; Aprotosoaie et al., 2016; Loureiro et al., 2017; Ramli, Yatim, Said, \& Hok, 2001).

\subsubsection{Alcaloides}

Los granos de cacao contienen alrededor del 4\% de alcaloides (Figura 8), siendo la teobromina (3,7-dimetilxantina) el alcaloide más abundante (2 - 3\%). El cacao constituye la mayor fuente natural de teobromina superando al té y el café; además, presenta pequeñas cantidades de cafeína (1,3,7- trimetilxantina) $\quad(0.2 \%$ aproximadamente) y trazas de teofilina. La teobromina y cafeína representan más del $99 \%$ de los alcaloides del grano, contribuyendo al sabor amargo típico del cacao y a la calidad sensorial de los productos alimenticios que lo contienen (Acierno et al., 2016; Afoakwa et al., 2008, 2011; Aprotosoaie et al., 2017; Araujo et al., 2014; Belščak, Komes, Horžić, Ganić, \& Karlović, 2009; Loureiro et al., 2017).<smiles>Cn1cnc2c1c(=O)[nH]c(=O)n2C</smiles>

(A)<smiles>Cn1c(=O)c2c(ncn2C)n(C)c1=O</smiles>

(B)<smiles>Cn1c(=O)c2[nH]cnc2n(C)c1=O</smiles>

(C)

Figura 8. Estructura química de las metilxantinas. Teobromina (A), cafeína (B), teofilina (C).

Fuente: Brunetto et al. (2007)

Estudios realizados por varios investigadores (Afoakwa et al., 2008; Brunetto et al., 2007; Diomande et al., 2015; Saltini, Akkerman, \& Frosch, 2013), demostraron que el contenido de teobromina, cafeína y la relación teobromina/cafeína (TBR/CAF) varía en función del genotipo de cacao. El contenido de teobromina encontrado en granos de cacao varió en el rango de 1.72 a $3.55 \%$ y se ha establecido que los mayores contenidos de este alcaloide se encuentran relacionados con cacao de tipo Forastero procedentes de Brasil (2.96 a 3.45\%) y África (3.02 a 3.45\%). Sin embargo, el cacao africano tiene menor contenido de cafeína (0.22 a $0.24 \%$ ) con relación al cacao Forastero de Brasil (0.51 a 0.59\%) (Tabla 4). Los cacaos de origen americano son 
más finos y más ricos en cafeína (0.30 a $0.60 \%$ ), mientras que, su contenido de teobromina varía en el rango de 2.85 a 3.43\% (Afoakwa et al., 2008).

Estudios realizados por Espín et al., (2007), reportaron que el cacao Nacional ecuatoriano procedente de fincas comerciales (híbridos Nacional x Trinitario) presentó contenidos promedio de teobromina y cafeína de $2.16 \% \pm 0.24$ y $0.38 \% \pm 0.04$; respectivamente. En clones locales de cacao Nacional se obtuvieron contenidos promedios de teobromina y cafeína de $2.25 \% \pm 0.22$ y $0.44 \% \pm 0.06$; en ese orden. En estudios realizados en cacao Nacional de diferentes regiones del Ecuador, con la finalidad de identificar componentes físicos, sensoriales y químicos que permitieran diferenciar al cacao por su calidad, reportó que el contenido de teobromina varía en el rango de 1.51 a $2.33 \%$, mientras que, la cafeína varía en el rango de 0.22 a $0.57 \%$ (Tabla 4). De igual manera estudios realizados por Brunetto et al. (2007), en cacao Criollo de Venezuela demostró que este genotipo presenta contenidos inferiores de teobromina $(0.65 \%)$ y cafeína $(0.38 \%)$ con relación a cacao de tipo Trinitario y Forastero (Jimenez, J., Amores, F., \& Solórzano et al., 2014).

En los últimos años varios estudios han demostrado que la relación teobromina /cafeína permite clasificar a las almendras de cacao por su origen genético (Trinitario, Forastero y Criollo); constituyendo una herramienta importante para discriminar la calidad de las almendras de diferentes orígenes geográficos. Las poblaciones de cacao procedentes de África presentaron contenidos menores de cafeína con relación a las almendras originarias de América, con valores de relación TBR/CAF de 39. En cacaos procedentes de Venezuela, Trinidad y Ecuador la relación T/C es de 6 aproximadamente (Afoakwa et al., 2008; Brunetto et al., 2007; Diomande et al., 2015; Espín et al., 2007; Saltini et al., 2013).

Los valores para la relación T/C varían según los grupos genéticos de cacao siendo mayor en almendras de tipo Forastero (15 - 10) que en muestras de Trinitario (10 - 5) y Criollo (2 - 1). Los resultados obtenidos por diferentes investigadores para materiales procedentes de África y Brasil, presentaron valores que lo ubican como cacao Forastero, mientras que las muestras procedentes de Ecuador (Espín et al., 2007; Samaniego et al., 2020) corresponden a cacao del grupo Trinitario; estos materiales son híbridos que surgieron a partir del cruzamiento de la variedad Nacional con materiales Trinitarios introducidos de Venezuela y Trinidad. Por otra parte, los valores obtenidos en materiales de origen venezolano los clasificaron como cacao del tipo 
Criollo (Tabla 4). Este índice constituye una herramienta de calidad muy importante, puesto que a nivel mundial se considera como cacaos finos a los que corresponden a los grupos Trinitario y Criollo, siendo los de tipo Forastero considerados como cacaos comunes o al granel, esta clasificación determina el precio del grano en el mercado internacional (Brunetto et al., 2007; Loureiro et al., 2017)

Tabla 4. Información resumida de alcaloides, contenido de teobromina y cafeína en cacao seco.

\begin{tabular}{|c|c|c|c|c|c|c|}
\hline Literatura & Origen & $\begin{array}{l}\text { Material } \\
\text { Genético }\end{array}$ & Estadístico & $\begin{array}{l}\text { Alcaloid } \\
\text { Teobromina } \\
\text { g/100g caca }\end{array}$ & $\begin{array}{l}\text { Ces } \\
\text { Cafeína } \\
\text { 10 seco }\end{array}$ & $\begin{array}{c}\text { Relación } \\
\text { Teobromina/Cafeína }\end{array}$ \\
\hline \multirow{5}{*}{$\begin{array}{c}\text { Palacios } \\
(2008)\end{array}$} & & & & \multicolumn{3}{|c|}{ Muestras $(\mathrm{n}=60)$} \\
\hline & $\begin{array}{l}\text { Colón } \\
\text { Eloy Alfaro }\end{array}$ & \multirow{4}{*}{$\begin{array}{l}\text { Híbridos } \\
\text { de cacao } \\
\text { Nacional }\end{array}$} & Promedio & 1.76 & 0.39 & 4.59 \\
\hline & $\begin{array}{l}\text { Esmeraldas } \\
\text { Naranjal } \\
\text { Guayas }\end{array}$ & & Promedio & 1.83 & 0.36 & 5.06 \\
\hline & época lluviosa & & Promedio & 1.72 & 0.35 & 4.88 \\
\hline & época seca & & Promedio & 1.87 & 0.40 & 4.77 \\
\hline \multirow{3}{*}{$\begin{array}{l}\text { Espín et al. } \\
(2007)\end{array}$} & & & & \multicolumn{3}{|c|}{ Muestras $(n=100)$} \\
\hline & $\begin{array}{l}\text { Guayas } \\
\text { Bolívar } \\
\text { Manabí }\end{array}$ & $\begin{array}{l}\text { Híbridos } \\
\text { de cacao } \\
\text { Nacional }\end{array}$ & Promedio & 2.16 & 0.38 & 5.68 \\
\hline & Los Ríos & $\begin{array}{l}\text { Clones de } \\
\text { cacao Nacio }\end{array}$ & $\begin{array}{l}\text { Promedio } \\
\text { nal }\end{array}$ & 2.25 & 0.44 & 5.11 \\
\hline & & & & \multicolumn{3}{|c|}{ Muestras $(n=11)$} \\
\hline \multirow{3}{*}{$\begin{array}{l}\text { Jiménez et al. } \\
\qquad(2014)\end{array}$} & Costa del Pacífico & Híbridos & Promedio & 1.79 & 0.32 & 5.59 \\
\hline & Bolívar & de cacao & Promedio & 2.16 & 0.46 & 4.70 \\
\hline & Región Amazónica & Nacional & Promedio & \multicolumn{3}{|c|}{ Muestras $(n=4)$} \\
\hline \multirow{2}{*}{$\begin{array}{l}\text { Brunetto et al. } \\
\text { (2007) }\end{array}$} & Mérida & Cacao & Promedio & 0.65 & 0.38 & 1.71 \\
\hline & Zulia & Criollo & Promedio & \multicolumn{3}{|c|}{ Muestras $(n=3)$} \\
\hline \multirow{3}{*}{$\begin{array}{c}\text { Amores et al. } \\
\text { (2009) }\end{array}$} & Ghana & Forastero & Promedio & 3.12 & 0.24 & 13.00 \\
\hline & Costa de Marfil & Forastero & Promedio & 2.92 & 0.27 & 10.81 \\
\hline & Nigeria & Forastero & Promedio & \multicolumn{3}{|c|}{ Muestras $(n=6)$} \\
\hline $\begin{array}{l}\text { Loureiro } \\
\text { (2012) }\end{array}$ & $\begin{array}{l}\text { Bahía } \\
\text { Brasil }\end{array}$ & Forastero & & 3.45 & $\begin{array}{c}0.51 \\
\text { Muestras }\end{array}$ & $n=36)$ \\
\hline $\begin{array}{c}\text { Loureiro } \\
(2014)\end{array}$ & $\begin{array}{l}\text { Región de Bahía } \\
\text { Brasil }\end{array}$ & $\mathrm{PH}-16$ & Promedio & 2.96 & 0.59 & 5.00 \\
\hline
\end{tabular}

Fuente: Brunetto et al., 2007; Espín et al., 2007; Jimenez, J., Amores, F., \& Solórzano, 2014; Loureiro et al., 2017

El contenido de teobromina y cafeína en los granos de cacao disminuye alrededor del 20-30\% durante el proceso de fermentación. La disminución de los alcaloides (20\%) no se da por transformaciones químicas durante la fermentación, esta se pierde por difusión en los tejidos y migración a los tegumentos; donde su contenido incrementa considerablemente. Esta pérdida de teobromina es en gran parte responsable de la disminución del amargor de las almendras bien fermentadas, y es por tanto responsable del sabor relativamente menos amargo del cacao (Aprotosoaie et al., 2016; Espín et al., 2007; Loureiro et al., 2017). 
Trabajos de investigación realizados en Ecuador, han demostrado que el contenido de teobromina en clones de cacao Nacional y muestras de fincas comerciales locales varió significativamente con el tiempo de fermentación, el genotipo y el año de cosecha. El contenido promedio de teobromina determinado en muestras de seis diferentes clones de cacao sometidos a un proceso de fermentación de 0 a 5 días varió entre $2.5 \%$ a $2.1 \%$ y para muestras de fincas comerciales los contenidos variaron de $2.2 \%$ a $1.8 \%$ (Figura 9). En los dos casos decrece el contenido de teobromina en un 20 a $25 \%$ durante la fermentación; lo cual confirma los resultados obtenidos descritos en los estudios citados (Espín et al., 2007; Jimenez, J., Amores, F., \& Solórzano, 2014).

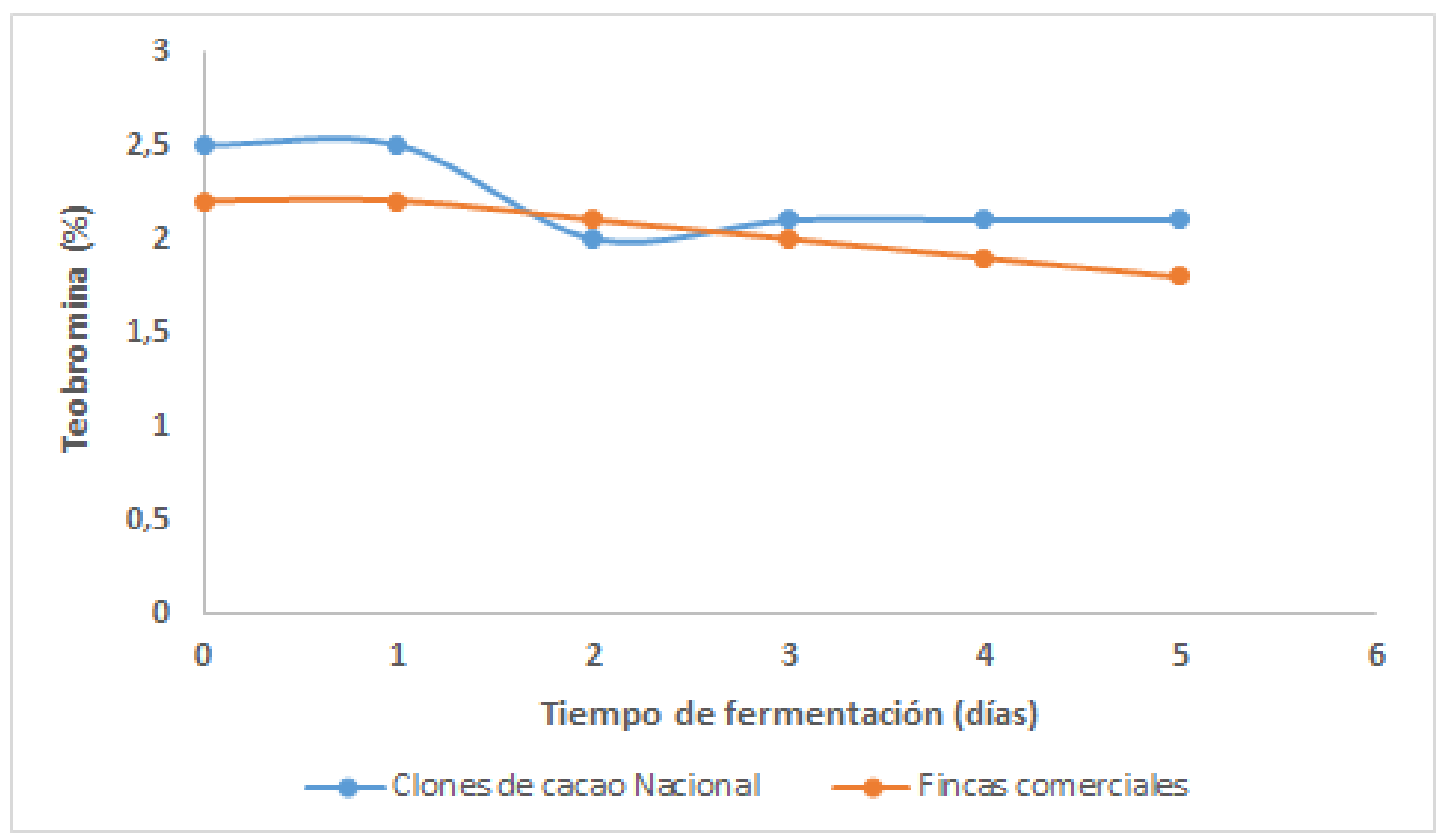

Figura 9. Efecto del proceso de fermentación sobre el contenido de teobromina en muestras de clones de cacao Nacional y fincas comerciales del Ecuador.

Fuente: Amores. et al. (2007)

Las metilxantinas presentes en los granos de cacao son moléculas psicofarmacológicamente activas que influyen en la estimulación del sistema nervioso central, efectos cardiovasculares y metabólicos, diuresis, estimulación de la secreción gástrica y en dosis altas estimulan de los músculos esqueléticos (Aprotosoaie et al., 2017; Franco-Agurto \& Suárez-Quirumbay, 2014).

Estas características hacen que el cacao sea un producto muy demandado a nivel mundial no solo por sus características sensoriales privilegiadas, sino también por sus 
diversos efectos benéficos para la salud. La cafeína es una metilxantina que mejora el rendimiento físico e intelectual, mitigan la fatiga y provocan una sensación de alerta (Aprotosoaie et al., 2016; Campos-Vega, Nieto-Figueroa, \& Oomah, 2018; do Carmo, Campos, da Silva, Abreu, \& Lopes, 2017).

La teobromina tiene un efecto más débil que la cafeína el cual disminuye en el caso de los productos de cacao, llegando a ser más baja o diferente (Aprotosoaie et al., 2017; Franco-Agurto \& Suárez-Quirumbay, 2014). El consumo de cacao y sus derivados representan solo una pequeña parte de la dieta humana y la concentración de metilxantinas es baja, por lo que, el consumo de estos alcaloides no constituye un riesgo para la salud humana (Aprotosoaie et al., 2016; Campos-Vega et al., 2018; Silva \& Pogǎ̌cnik, 2020).

\subsubsection{Compuestos fenólicos}

\subsubsection{Definición y estructura}

Los compuestos fenólicos constituyen uno de los grupos más numerosos y más ampliamente distribuidos en el reino vegetal, con más de 8000 estructuras fenólicas conocidas (Quiñones, Miguel, \& Aleixandre, 2012). Son producto del metabolismo secundario de las plantas y surgen biogenéticamente a partir de dos vías principales de síntesis: la vía del ácido shiquímico (Figura 10) y la vía del acetato, mismos que, se derivan del metabolismo de la glucosa (Gutiérrez Maydata, 2002; Loureiro et al., 2017; Ribeiro et al., 2019).

Los compuestos fenólicos cumplen funciones importantes dentro de los procesos fisiológicos de las plantas como la respiración, el crecimiento y los mecanismos de defensa (protección frente al ataque de patógenos o herbívoros); además, constituyen los pigmentos de las flores, frutos y tallos. Su estructura química está formada por anillos aromáticos, grupos hidroxilo y dobles enlaces conjugados a partir de los cuales ejercen una acción antioxidante. Los compuestos fenólicos se pueden dividir en al menos 10 clases diferentes dependiendo de su estructura básica, así se han identificado los siguientes grupos hasta la fecha: los fenoles simples, benzoquinonas, los ácidos fenólicos, ácidos fenilacéticos, acetofenonas, ácidos hidroxicinámicos, fenilpropanos y cumarinas, naftoquinonas, xantonas, estilbenos y antraquinonas, flavonoides, lignanos y ligninas (Colizzi, 2019; Gómez-Juaristi et al., 2011; Gutiérrez Maydata, 2002; Quiñones et al., 2012; Wollgast \& Anklam, 2000). 


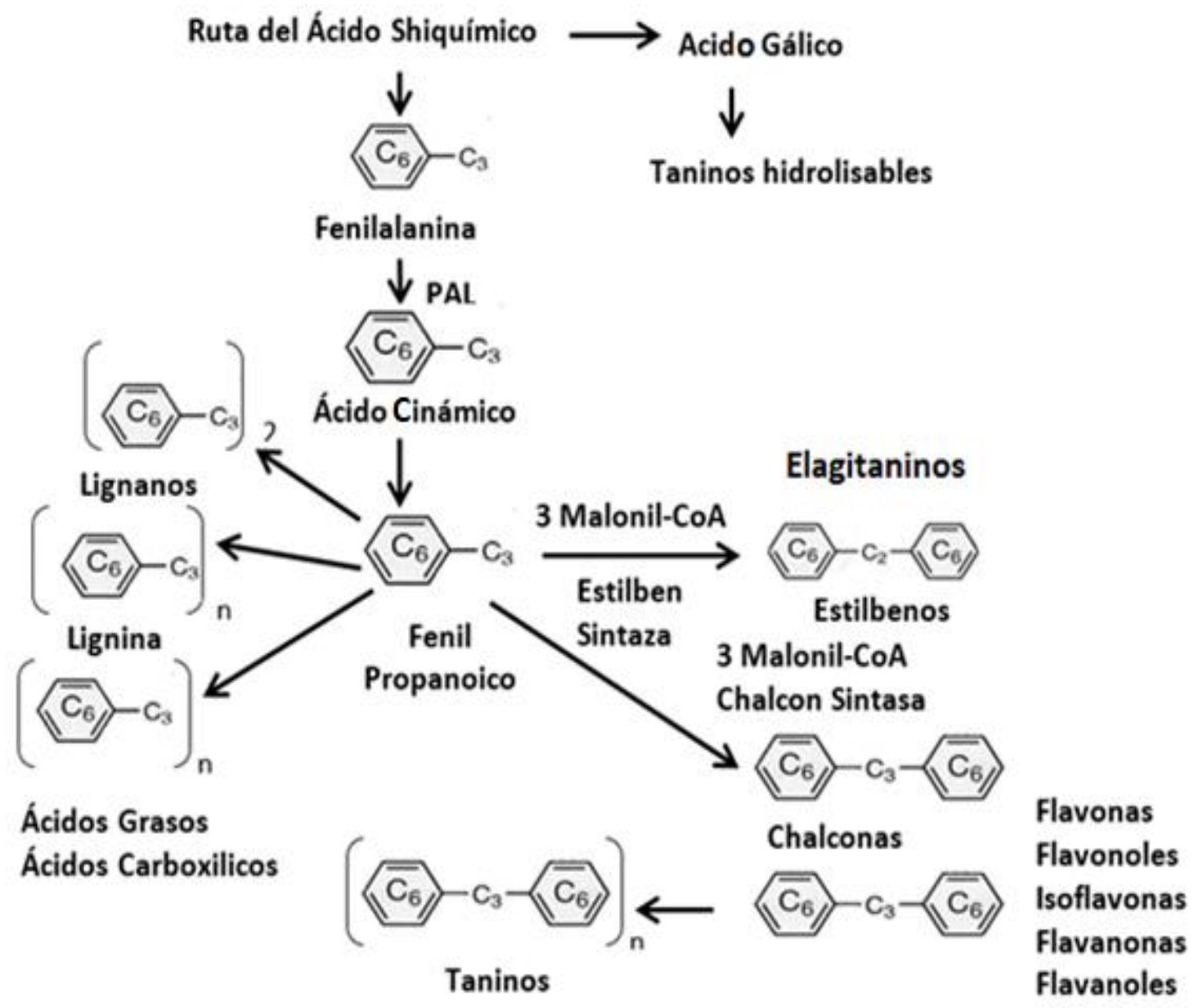

Figura 10. Producción de flavonoides y estilbenos a partir de Cumaril-CoA y de MalonilCoA.

Fuente: Szajdek \& Borowska, (2008)

\subsubsection{Los compuestos fenólicos en los alimentos}

Los compuestos fenólicos se distribuyen ampliamente en diferentes variedades de frutas, verduras, nueces, semillas, flores, cortezas, así como en alimentos y bebidas elaboradas con ingredientes naturales ricos en los mismos. El cacao, las manzanas, el té, el café, el vino, las mermeladas o las cebollas son fuentes comunes de polifenoles en la dieta humana (Serra, Almeida, \& Dinis, 2019).

Tradicionalmente, el interés principal se ha centrado en sus propiedades organolépticas en los alimentos, como el color (antocianos y curcumina (Kim et al., 2011; Szajdek \& Borowska, 2008). Pero durante las últimas décadas han sido cada vez más reconocidos por su valor nutricional y funcional. De hecho, podrían ayudar a reducir el riesgo de contraer enfermedades crónicas y en general tienen un efecto 
positivo en la salud. Se ha identificado que ciertos polifenoles pueden mostrar efectos anticarcinogénicos, antiaterogénicos, antitrombóticos, antialergénicos, inmunomoduladores, antimicrobianos, vasodilatadores y analgésicos (Aprotosoaie et al., 2017; Hartel et al., 2018; Kim et al., 2011; Sudano et al., 2012; Szajdek \& Borowska, 2008; Wollgast \& Anklam, 2000).

A nivel industrial, estas propiedades de los compuestos fenólicos recientemente descubiertos han sido explotadas para la fabricación de cosméticos, medicamentos, productos farmacéuticos, suplementos nutricionales o alimentos funcionales. La funcionalidad para la salud de estos productos está estrechamente relacionada con su contenido de polifenoles, que generalmente es superior a los productos tradicionales (leches enriquecidas con isoflavonas, chocolates enriquecidos con procianidinas, etc.) (Gómez-Juaristi et al., 2011; Predan et al., 2019; Ribeiro et al., 2019; Sudano et al., 2012).

\subsubsection{Los polifenoles en el cacao}

El cacao es una fuente extraordinariamente rica de polifenoles, su contenido en los granos es similar al del vino, el té o las verduras; sin embargo, su identificación y cuantificación se ha desarrollado recientemente (Afoakwa et al., 2008; Aprotosoaie et al., 2016; Krähmer et al., 2015; Wollgast \& Anklam, 2000).

Los polifenoles de las almendras de cacao se almacenan en las células pigmentarias del parénquima de los cotiledones. Según la cantidad de antocianos presentes en las células de almacenamiento de polifenoles, los granos pueden ser de color blanco a violeta intenso. Además del color, estos compuestos le confieren notas sensoriales astringentes, amargas y contribuyen de manera significativa a la formación de los sabores verdes y afrutados de los licores de cacao. Por otro lado, los polifenoles son responsables de los beneficios positivos para la salud asociados con el consumo de cacao (Aprotosoaie et al., 2016; Wollgast \& Anklam, 2000). 
<smiles>Oc1cc(O)c2c(c1)O[C@H](c1ccc(O)c(O)c1)C(O)C2</smiles>

A<smiles>Oc1cc(O)c2c(c1)O[C@H](c1cc(O)c(O)c(O)c1)C(O)C2</smiles><smiles>Oc1cc(O)c2c(c1)OC(c1ccc(O)c(O)c1)C(O)C2</smiles><smiles>Oc1cc(O)c2c(c1)OC(c1cc(O)c(O)c(O)c1)C(O)C2</smiles>

Figura 11. Estructura química de flavan-3-oles del cacao: (+)-catequina (A), (-)epicatequina (B), (+)-galocatequina (C), (-)-epigalocatequina (D).

Fuente: Kim et al. (2011)

En el cacao se distinguen tres grupos principales de polifenoles; las catequinas (flavan-3-oles), antocianos y proantocianidinas, representado los monómeros del 5 al $10 \%$ del total de polifenoles y los polímeros el $90 \%$, aproximadamente (Hue et al., 2014). Del total de los polifenoles, el 29 al 38\% pertenecen al grupo de catequinas como (-)-epicatequina (35\%; Figura 11), (+)-catequina, (+)-galocatequina y (-)epigalocatequina. La fracción de antocianos representa aproximadamente el $4 \%$ de los polifenoles totales y está formada por leucoantocianidinas L1, L2, L3 y L4, cianidina 3$\alpha$-L-arabinósido y cianidina 3- $\beta$-D-galactósido (Castro et al., 2020; de Souza et al., 2018; Gutiérrez Maydata, 2002; Kim et al., 2011; Ribeiro et al., 2019).

Las proantocianidinas (Figura 12) representan aproximadamente el 58 al $65 \%$ del total de polifenoles del cacao. Estos polímeros están formados por unidades de flavan-3oles unidos por enlaces 4 - 8 o 4 - 6 (Kim et al., 2011; Loureiro et al., 2017; Patras, Milev, Vrancken, \& Kuhnert, 2014; Steinberg, Bearden, \& Keen, 2003). En este grupo de polifenoles predominan los dímeros a hexámeros, encontrándose polímeros de hasta 18 unidades monoméricas en los productos del cacao. Las proantocianidinas más importantes son los dímeros $\mathrm{B}_{1}, \mathrm{~B}_{2}, \mathrm{~B}_{3}, \mathrm{~B}_{4}$ y $\mathrm{B}_{5}$, trímeros $\mathrm{C}_{1}$ y tetrámeros $\mathrm{D}$. Otros compuestos fenólicos menores incluyen las flavonas (apigenina, luteolina y sus glucósidos), los flavonoles (kaempferol y sus glicósidos) y ácidos fenólicos (ácido caféico, ácido clorogénico, ácido cumárico, ácido ferúlico y ácido siríngico) (de Souza et al., 2018; Fernández, Yee, Sulbarán, \& Peña, 2014; Hue et al., 2014; Samaniego et al., 2020; Yépez Rivadeneira, 2017). 


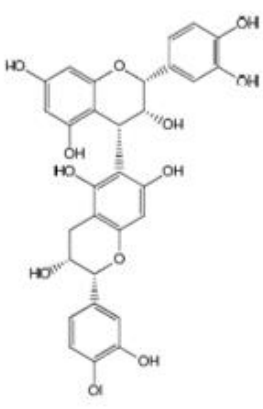

A

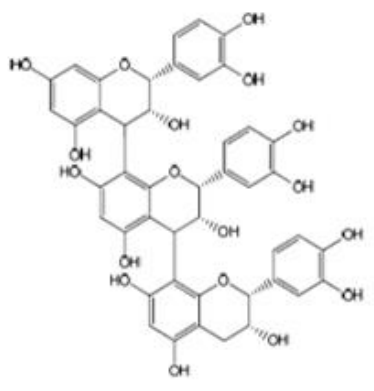

B

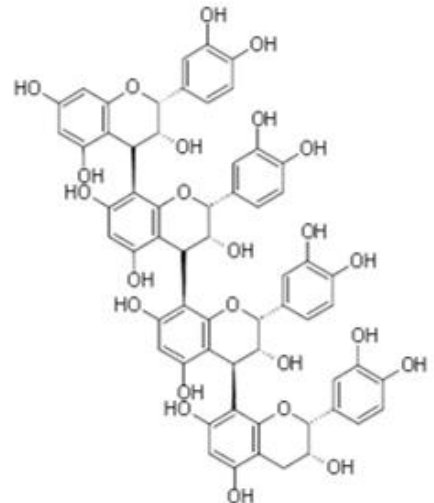

C

Figura 12. Principales proantocianidinas del cacao: procianidina $B 5(A)$, procianidina $C 1$ (B) y procianidina $D(C)$.

Fuente: Kim et al. (2011)

La cantidad total de compuestos fenólicos presentes en los granos de cacao varía de 15 al $20 \%$ de materia seca desengrasada. Esto equivale aproximadamente al $6 \%$ en granos de cacao secos, con $54 \%$ de grasa y $6 \%$ de agua. En los granos fermentados y secos corresponde aproximadamente al $5 \%$ de su peso, un contenido de $10 \%$ o más se considera como un signo de mala fermentación. Estos contenidos se consideran válidos para cacao tipo Forastero, por otro lado; los granos de cacao Criollos presentan aproximadamente 2/3 de esta cantidad de polifenoles (de Souza et al., 2018; Kim et al., 2011; Wollgast \& Anklam, 2000)

En este contexto, se ha establecido que el contenido y la composición de los compuestos fenólicos varían mucho dependiendo de varios factores como: el genotipo, el origen, el estado de madurez, tratamiento poscosecha y procesamiento de las almendras. Así, por ejemplo; el genotipo de cacao Criollo presenta un alto contenido de procianidinas, mientras que los cultivares Trinitario y Forastero muestran concentraciones más bajas de este tipo de polifenoles. Las antocianos proporcionan el color específico a los granos del tipo Forastero, pero no en el tipo de cacao Criollo. El procesamiento de los granos puede conducir a una pérdida significativa de flavan-3oles; (+)-catequina y (-)-epicatequina (del $100 \%$ al $10 \%$ en chocolate) (CAOBISCO/ECA/CFC, 2015; de Souza et al., 2018; Grimbs \& Ullrich, 2017; Gutiérrez Maydata, 2002; Hartel et al., 2018; Lim, 2012; Patras et al., 2014; Samaniego et al., 2020; Todorovic et al., 2015; Yépez Rivadeneira, 2017). 
Tabla 5. Contenido de polifenoles en cacao seco de diferentes orígenes (Parte 1)

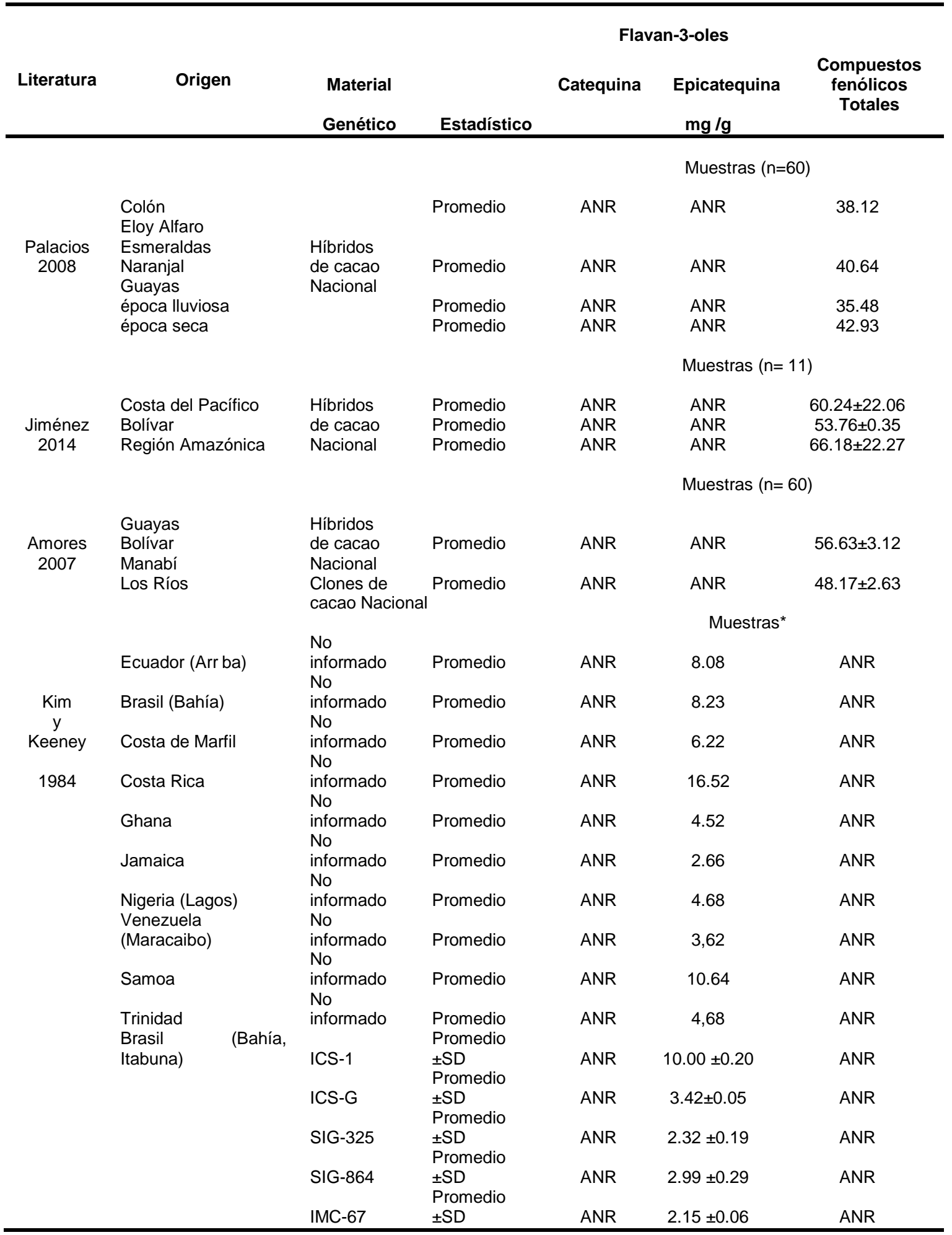

SD: Desviación estándar; AND: Análisis no realizados; IAC: Selección del Instituto Agronómico de Campiñas; ICS: Imperial College Selection;

IMC: Mezcla lquito calabasillo; SCA: Scavina; UF: Fruta unida

DBCA: Muestras evaluadas en un diseño de bloques al azar (2 regiones $X 5$ fincas $X 3$ tiempos de fermentación)

* Promedio de dos inyecciones de un mismo extracto

** Muestras con fermentación convencional por 7 días 


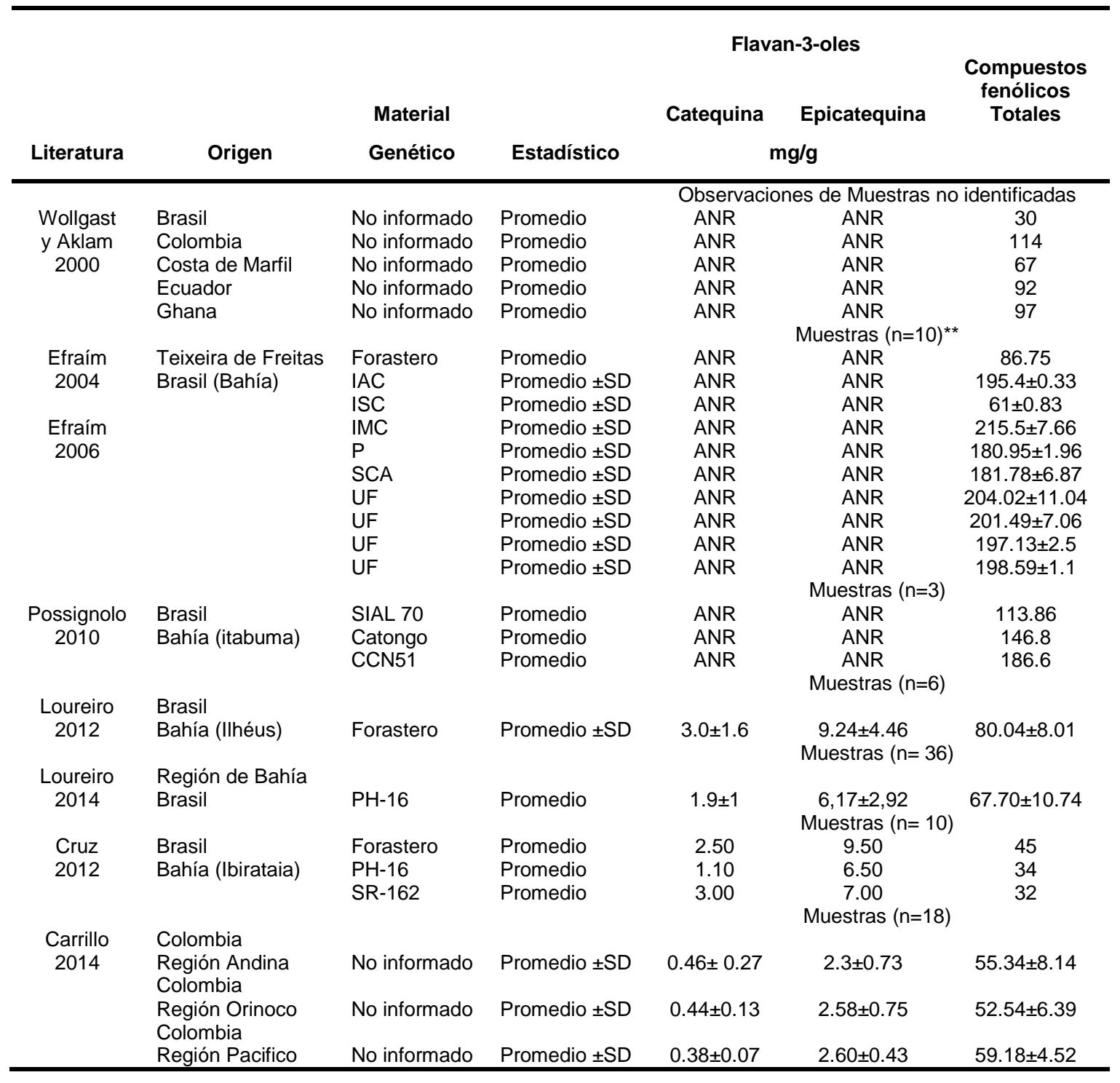

SD: Desviación estándar; AND: Análisis no realizados; IAC: Selección del Instituto Agronómico de Campiñas; ICS: Imperial College Selection;

IMC: Mezcla lquito calabasillo; SCA: Scavina; UF: Fruta unida

DBCA: Muestras evaluadas en un diseño de bloques al azar (2 regiones $X 5$ fincas $X 3$ tiempos de fermentación)

* Promedio de dos inyecciones de un mismo extracto

** Muestras con fermentación convencional por 7 días

Fuente: Brunetto et al., 2007; Espín et al., 2007; Jimenez, J., Amores, F., \& Solórzano, 2014; Loureiro et al., 2017

Según el trabajo realizado por Amores. et al. (2007), los contenidos de compuestos fenólicos totales determinados en muestras fermentadas y secas de seis clones de cacao Nacional y muestras procedentes de cuatro fincas comerciales locales (Híbridos de cacao Nacional x Trinitario) de Ecuador, variaron en el rango de 53.2 a 59.3 y de 46.0 a $51.5 \mathrm{mg}$ acido gálico/g respectivamente. 
Estudios realizados por Jiménez et al. (2014), reportaron que el cacao Nacional fermentado y seco de diferentes regiones del Ecuador, presenta un contenido de compuestos fenólicos totales que varía en el rango de 34,67 a 100,05 mg ácido gálico/g. Sin embargo, las muestras de híbridos de cacao Nacional ecuatoriano de las provincias de Esmeraldas y Guayas presentaron contenidos bajos de polifenoles totales en un rango de 35,83 a 40,64 mg ácido gálico/g, como se muestra en la Tabla 5.

De acuerdo al estudio comparativo presentado en la Tabla 5, el contenido de compuestos fenólicos totales observado en muestras de cacao brasileño ( $30 \mathrm{mg} / \mathrm{g}$ ) fue 3 a 4 veces menor que los obtenidos en cacao de Ghana (97 mg/g), Ecuador (92 $\mathrm{mg} / \mathrm{g}$ ) y Colombia (114 mg/g). Sin embargo, en estudios más recientes se encontraron valores de compuestos fenólicos totales superiores a $215 \mathrm{mg} / \mathrm{g}$ en muestras procedentes de Brasil.

En el caso del contenido de los flavan-3-oles (+)-catequina y (-)-epicatequina existe información muy escasa para muestras de cacao de origen ecuatoriano y latinoamericano. En cacao Forastero de Brasil se reportaron contenidos de (+)catequina en el rango de 1.1 a $3 \mathrm{mg} / \mathrm{g}$, mientras que, en muestras de cacao tipo Trinitario de Colombia los contenidos de este polifenol variaron en el rango de 0.38 a $0.46 \mathrm{mg} / \mathrm{g}$ (Tabla 5). La (-)-epicatequina es el polifenol más abundante del cacao (9.1$28 \%$ ) presentó contenidos de 2.30 a $2.60 \mathrm{mg} / \mathrm{g}$ y de 6.17 a $9.50 \mathrm{mg} / \mathrm{g}$ para cacao de Brasil y Colombia respectivamente (Tabla 5). El contenido de (-)-epicatequina también varía en función del origen geográfico, es así que en cacao procedente de Jamaica, Costa de Marfil y Ecuador se reportaron contenidos de $2.66 ; 6.22$ y $8.08 \mathrm{mg} / \mathrm{g}$ respectivamente (Tabla 5) (Carrillo, Londoño-Londoño, \& Gil, 2014; Loureiro et al., 2017; Wollgast \& Anklam, 2000).

Durante el tratamiento poscosecha (fermentación y secado) y las etapas posteriores de transformación, los polifenoles intervienen en reacciones bioquímicas complejas que son importantes para la formación del sabor, aroma y el color del cacao. Durante la fermentación los polifenoles se difunden de las células y se someten a reacciones de oxidación y condensación, esto conduce a la formación de polímeros de alto peso molecular (la mayor parte taninos insolubles) (de Souza et al., 2018; Gibson \& Newsham, 2018; Hue et al., 2014; Kongor et al., 2016; Santos et al., 2020; Wollgast \& Anklam, 2000). 
Los antocianos son hidrolizados en antocianidinas y se polimerizan con catequinas simples, disminuyendo su contenido durante el proceso de fermentación (93 a 90\%) a partir del 4 día de fermentación, lo que muestra un buen índice del grado de fermentación de las almendras de cacao. Los pigmentos poliméricos (melaninas) formados en esta reacción, les confieren a los granos el color marrón típico del chocolate (Caligiani, Marseglia, Prandi, \& Palla, 2016; de Souza et al., 2018; Hue et al., 2014; Kim et al., 2011; Kongor et al., 2016; Lim, 2012; Loureiro et al., 2017; Santos et al., 2020; Wollgast \& Anklam, 2000).

Estudios realizados por Hue et al. (2014), en cacao de diferentes orígenes del mundo han demostrado que el contenido de polifenoles disminuye durante la fermentación. A pesar de que, el proceso de fermentación se realiza en todo el mundo, este no está estandarizado, por lo tanto, el contenido de polifenoles en los granos de cacao al final de la fermentación varía en el rango de 2 a $15 \mathrm{mg} / \mathrm{g}$ (Figura 13), lo cual influye sobre la calidad final de los granos que son utilizados principalmente para la fabricación de chocolates.

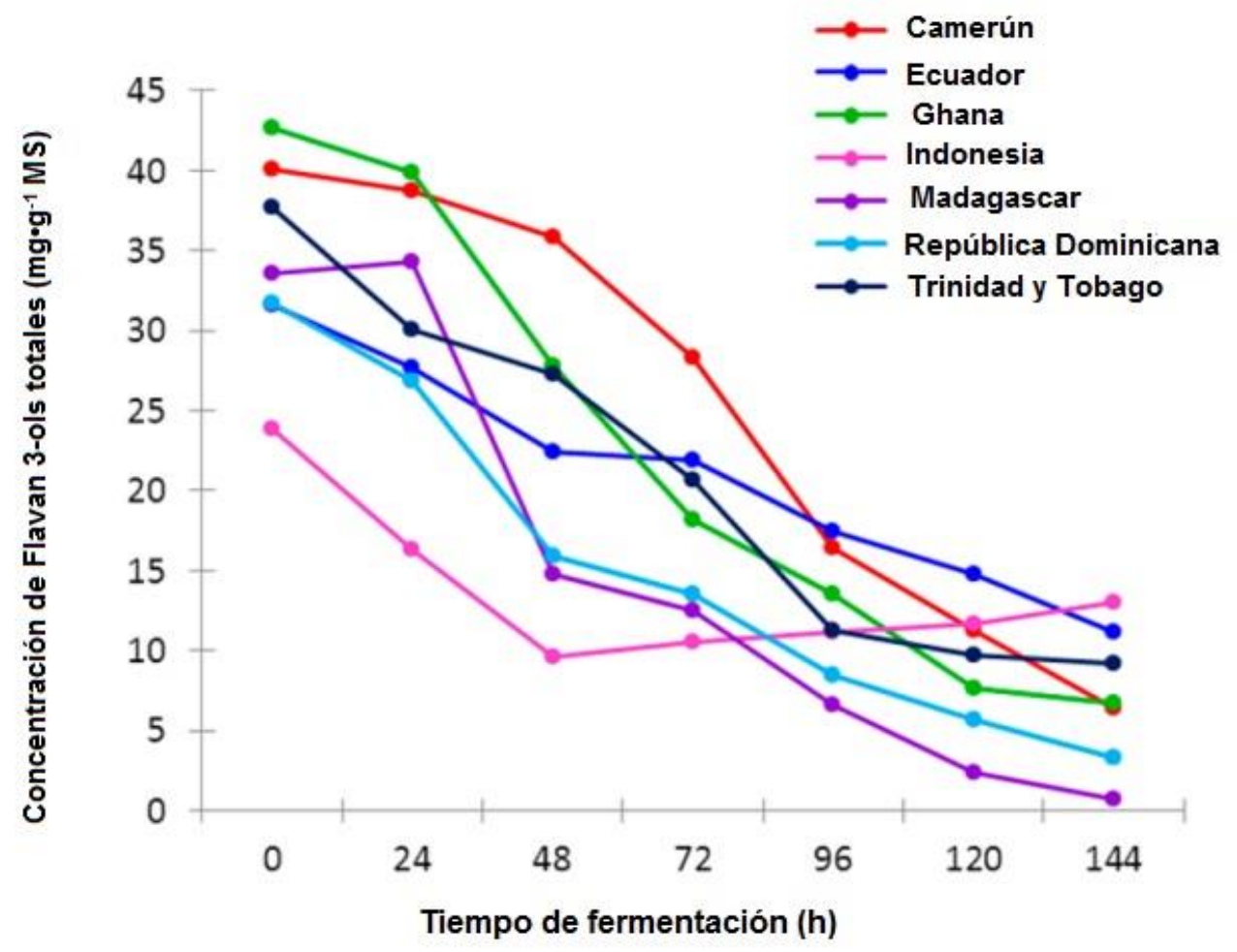

Figura 13. Efecto de la fermentación sobre el contenido de polifenoles en cacao de diferentes orígenes.

Fuente: Hue et al. (2014) 
Por otro lado, estudios recientes indican que el contenido de proantocianidinas disminuye de 3 a 5 veces durante el proceso de fermentación, evidenciándose una correlación negativa entre el contenido de procianidinas con el grado de fermentación y el cambio de coloración de violeta a marrón en lo granos de cacao. Estas reacciones de condensación se confirman por una fuerte disminución del contenido de (-)epicatequina del 10 a 20\%, aproximadamente (Figura 14). Sin embargo, esta disminución no se produce solamente por reacciones de condensación, sino también por la difusión de los polifenoles en los lixiviados de la fermentación (Hue et al., 2014; Wollgast \& Anklam, 2000)

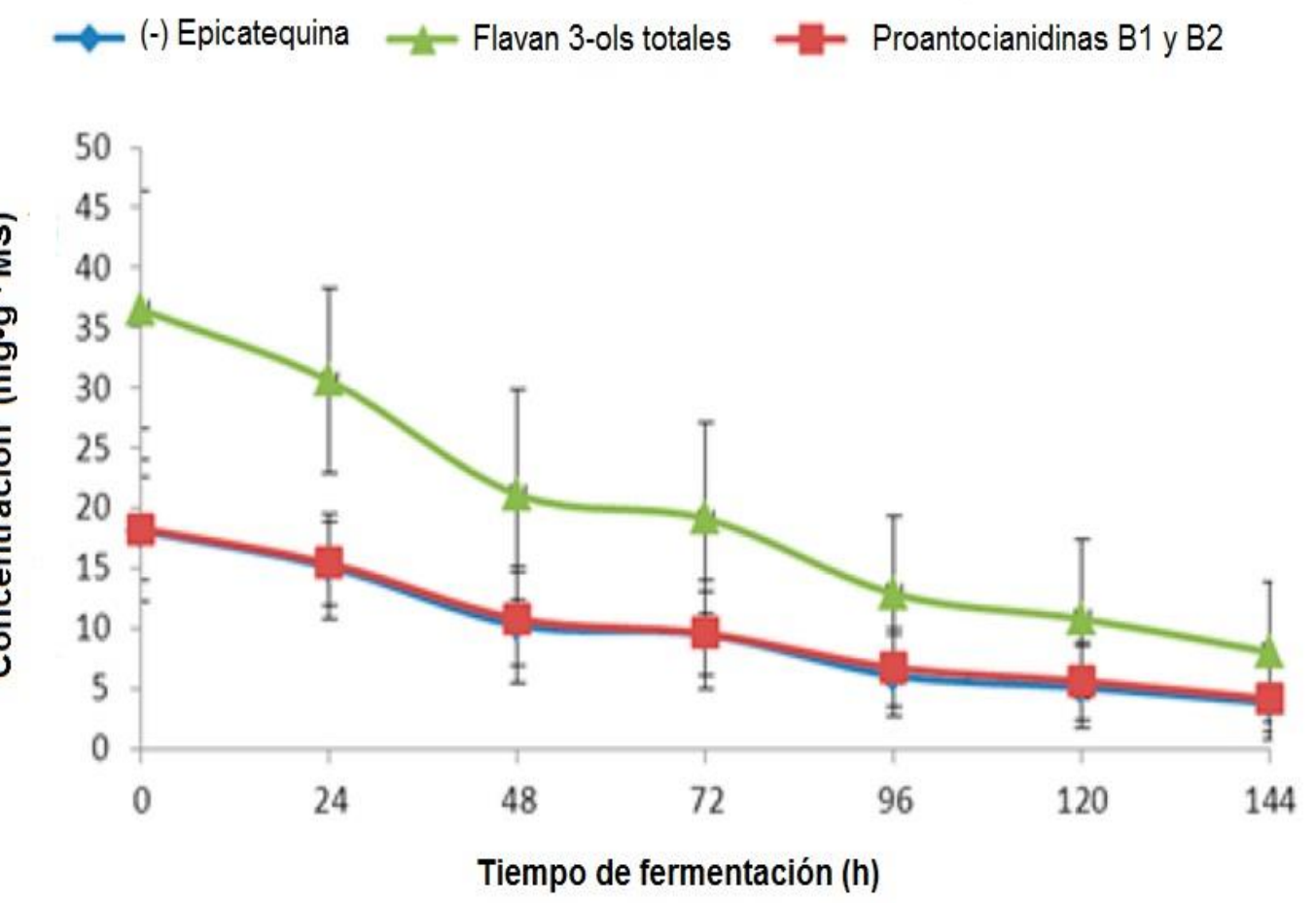

Figura 14. Efecto de la fermentación sobre el contenido de (-)- epicatequina y proantocianidinas $B_{1}$ y $B_{2}$ del cacao.

Fuente: Hue et al., (2014)

Numerosos autores (Campos-Vega et al., 2018; CAOBISCO/ECA/CFC, 2015; Carrillo et al., 2014; Castro et al., 2020; de Souza et al., 2018; Fernández et al., 2014; Gibson \& Newsham, 2018; Gómez-Juaristi et al., 2011; Gutiérrez Maydata, 2002; Hartel et al., 2018; Kim et al., 2011; Loureiro et al., 2017; Martínez et al., 2012; Samaniego et al., 2020) se han interesado por la investigación de los polifenoles de los granos de cacao y del chocolate, netamente en razón de su efecto benéfico para la salud y desde 1984 se ha demostrado que existe diferencias en el contenido de (-)-epicatequina en las almendras de cacao fermentadas o no, en función del origen geográfico; las cuales 
son atribuidas a los diferentes niveles de fermentación de los granos, además se ha demostrado que la capacidad antioxidante ligada a la presencia de polifenoles varía en función del origen geográfico de los granos.

En los últimos 10 años se ha puesto un interés especial en la investigación de los compuestos fenólicos en cacao y productos derivados como el chocolate. Se ha demostrado que la ingestión regular de polvo de cacao permite aumentar la tasa plasmática del HDL (Hight Density Lipoproteins, por sus siglas en inglés). Además, evita la oxidación del LDL (Low density Lipoproteins, por sus siglas en inglés), dicho fenómeno es netamente responsable de la aterosclerosis. Los resultados de los estudios in vitro establecieron que las procianidinas del cacao son poderosos antioxidantes y quelantes de hierro y cobre, por lo tanto, reducen la oxidación de LDL. Estás estimulan la producción de óxido nítrico a nivel del endotelio de las arterias, lo que limita igualmente el desarrollo de la aterosclerosis (Aprotosoaie et al., 2016; Gómez-Juaristi et al., 2011; Ludovici et al., 2017; Martín \& Ramos, 2016; Pereira-Caro et al., 2013; Quiñones et al., 2012; Ribeiro et al., 2019; Serra et al., 2019; Sudano et al., 2012).

Otros estudios recientes realizados en personas hipertensas demostraron que un consumo ligero de chocolate (6.3 $\mathrm{mg} / \mathrm{día})$ permiten una ligera disminución de la presión sistólica sin pérdida de peso, lo que disminuye sensiblemente los riesgos de tener ataques cerebrales o cardiacos. Por otra parte, los efectos de las procianidinas y los flavanoles del cacao y del chocolate han sido probados sobre diversos tipos de células cancerosas humanas, ejerciendo un efecto anti proliferativo sobre células malignas del colon y seno (Aprotosoaie et al., 2016; Lim, 2012; Patras et al., 2014)

En este contexto, debido a que los metabolitos secundarios del cacao producen efectos benéficos para la salud humana, se han intensificado estudios que incluyan dentro de sus diseños experimentales, la relación que existe entre el contenido de estas sustancias con las condiciones edafoclimáticas de producción. En Ecuador, se ha demostrado que los valores de polifenoles totales son más altos en los granos de cacao producidos en la época seca y el contenido de teobromina es menor en la temporada de Iluvia (Espín et al., 2007; Konger Edem et al., 2016; Loureiro et al., 2017; Samaniego et al., 2020) 


\subsubsection{Lípidos}

La fracción lipídica es el componente químico más abundante en los granos de cacao, su composición dependerá de ciertos factores como la variedad, las condiciones ambientales de producción, el estado de madurez de los frutos y la edad de la plantación. La manteca de cacao puede alcanzar alrededor del 48 al 57\% del peso total de los granos secos y se destaca como el componente más importante de los granos, porque de su calidad (color y aroma) depende de las características físicas, la estabilidad del chocolate y su precio en el mercado (CAOBISCO/ECA/CFC, 2015; D'Souza et al., 2017; do Carmo et al., 2017; Jahurul et al., 2013; Loureiro et al., 2017; Naik \& Kumar, 2014; Servent et al., 2018).

Al evaluar muestras fermentadas y secas de seis clones de cacao Nacional y de cuatro fincas comerciales locales (Híbridos de cacao Nacional x Trinitario) de Ecuador; Amores et al. (2007), determinó que el contenido de grasa varía en el rango de 48.7 a $50.6 \%$ y 47.1 a $49.0 \%$ respectivamente. Por su origen geográfico y la variedad de cacao, estos valores concuerdan con los contenidos (45 a 57\%) reportados por otros autores para muestras procedentes de países de Sur y Centroamérica (Tabla 6).

Jimenez et al., (2014), en estudios realizados en cacao Nacional fermentado y seco de diferentes regiones del Ecuador, reportó que el contenido de grasa total varía en el rango de 42.28 a 47,33\%, mientras que Palacios 2008, reportó que el contenido de grasa total evaluada en muestras de híbridos de cacao Nacional ecuatoriano de las provincias de Esmeraldas y Guayas varía en el rango de 46.62 a 47.9 \% (Tabla 6).

La grasa del cacao es altamente demandada y su precio en el mercado es superior en comparación con otras grasas y aceites vegetales, debido a sus propiedades físicas (reología y textura), químicas y organolépticas (CAOBISCO/ECA/CFC, 2015; Jahurul et al., 2013; Riaño et al., 2016; Torres-Moreno et al., 2015). Está constituida por el $97 \%$ de glicerolípidos, principalmente triacilgliceroles ( $\geq 70 \%)$ y trazas de fosfolípidos, mono y diglicéridos (Tabla 7)(CAOBISCO/ECA/CFC, 2015; Jahurul et al., 2013; Naik \& Kumar, 2014; Riaño et al., 2016; Servent et al., 2018; Torres-Moreno et al., 2015). 
Tabla 6. Información resumida del contenido de grasa en granos de cacao seco

\begin{tabular}{|c|c|c|c|c|}
\hline Literatura & Origen & $\begin{array}{l}\text { Material } \\
\text { Genético }\end{array}$ & Estadístico & $\begin{array}{l}\text { Grasa Total } \\
\mathrm{g} / 100 \mathrm{~g}\end{array}$ \\
\hline & & & & Muestras $(n=2)$ \\
\hline Acosta & Venezuela & Criollo & Promedio & 50.99 \\
\hline \multirow[t]{3}{*}{2001} & Cumboto, Aragua & Forastero & Promedio & 45.52 \\
\hline & & Trinitario & Promedio & 52.24 \\
\hline & & & & Muestras $(n=2)$ \\
\hline Efraím & Brasil (Bahía) & Forastero & Promedio & 50.43 \\
\hline \multirow[t]{2}{*}{2004} & Teixeira de Freitas & & & \\
\hline & & & & Muestras (ONI) \\
\hline Álvarez & Venezuela & & Promedio $\pm S D$ & $56 \pm 1.8$ \\
\hline \multirow[t]{6}{*}{2007} & Cuyagua & & Promedio $\pm S D$ & $54.44 \pm 1.1$ \\
\hline & & & Promedio $\pm S D$ & $56.07 \pm 1.4$ \\
\hline & & & Promedio $\pm S D$ & $54,61 \pm 3,2$ \\
\hline & & & Promedio $\pm S D$ & $55.41 \pm 0.0$ \\
\hline & & & Promedio $\pm S D$ & $56.01 \pm 0.1$ \\
\hline & & & & Muestras $(n=115)$ \\
\hline Khant & International & Trinitario & Promedio $\pm S D$ & $52.46 \pm 1.9$ \\
\hline \multirow[t]{5}{*}{2008} & Cocoa Genebank & Alto Amazónico & Promedio $\pm S D$ & $52.50 \pm 4.9$ \\
\hline & Trinidad & Forastero & Promedio $\pm S D$ & $54.46 \pm 1.2$ \\
\hline & & & & Muestras evaluadas en DBCA \\
\hline & Esmeraldas & & Promedio & 47.91 \\
\hline & Colón, Eloy Alfaro & Híbridos & & \\
\hline Palacios & Guayas & de cacao & Promedio & 46.62 \\
\hline \multirow[t]{5}{*}{2008} & Naranjal & Nacional & & \\
\hline & época lluviosa & & Promedio & 46.57 \\
\hline & época seca & & Promedio & 47.97 \\
\hline & & & & Muestras $(n=11)$ \\
\hline & Costa del Pacífico & Híbridos & Promedio $\pm S D$ & $45.64 \pm 1.1$ \\
\hline Jiménez & Bolívar & de cacao & Promedio $\pm S D$ & $45.64 \pm 1.5$ \\
\hline \multirow[t]{3}{*}{2014} & Región Amazónica & Nacional & Promedio $\pm S D$ & $45.41 \pm 0.72$ \\
\hline & & & & Muestras $(n=60)$ \\
\hline & Guayas & Híbridos de & & \\
\hline Amores & Bolívar & cacao Nacional & Promedio $\pm S D$ & $47.60 \pm 0.9$ \\
\hline \multirow[t]{3}{*}{2007} & Manabí & Clones de & Promedio $\pm S D$ & $49.15 \pm 0.8$ \\
\hline & Los Ríos & cacao Nacional & & \\
\hline & & & & Muestras $(n=3)$ \\
\hline & Teixeira de Freitas & Forastero & Promedio & $55.6 \pm 0.1$ \\
\hline \multirow[t]{2}{*}{2010} & Brasil (Bahía) & & & \\
\hline & & & & Muestras $(n=10)$ \\
\hline Cruz & Brasil & Forastero & Promedio & $48.03 \pm 0.4$ \\
\hline \multirow[t]{3}{*}{2012} & Bahía (Ibirataia) & $\mathrm{PH}-16$ & Promedio & $45.75 \pm 5.0$ \\
\hline & & SR-162 & Promedio & $48.68 \pm 3.7$ \\
\hline & & & & Muestras $(n=3)$ \\
\hline $\begin{array}{l}\text { Afoakwa } \\
2013\end{array}$ & $\begin{array}{l}\text { Cocoa Research Institute of Ghana } \\
\text { (CRIG). Tafo, Ghana }\end{array}$ & $\begin{array}{l}\text { Mezcla } \\
\text { Híbridos }\end{array}$ & $\begin{array}{l}\text { Promedio } \pm S D \\
\text { (rango) }\end{array}$ & $55.4 \pm 0.6-50.4 \pm 0.5$ \\
\hline
\end{tabular}

DBCA: Diseño de bloques completos al azar; 2 (regiones) X 3 (tiempos de fermentación) X 5 (fincas) dos bloques; época seca y época lluviosa. 4 repeticiones

Fuente: Loureiro et al., 2017; de Souza et al., 2018; Lim, 2012a

Las propiedades fisicoquímicas especiales de la manteca de cacao están determinadas por su composición y disposición específica de ácidos grasos. Estás le confieren sus propiedades como el grado de cristalización y sus características de fusión en la boca, lo que provoca un efecto refrescante y la sensación típica que hace de esta grasa el principal ingrediente de la industria de chocolatería y confitería. 
Además, es muy adecuada para la fabricación de una amplia variedad de productos en la industria cosmética y farmacéutica (CAOBISCO/ECA/CFC, 2015; Jahurul et al., 2013; Riaño et al., 2016; Servent et al., 2018).

Tabla 7. Perfil de ácidos grasos y triglicéridos en grasa de cacao

\begin{tabular}{ll}
\hline \multicolumn{1}{c}{ Tipo de ácido graso } & \% de ácido graso \\
\hline Ácidos grasos saturados & $57-64$ \\
Ácido Palmítico (C16:0) & $24.5-40.2$ \\
Ácido Esteárico (C18:0) & $33.7-40.2$ \\
Ácido Mirístico (C14:0) & $0-4$ \\
Ácido Araquídico (C20:0) & 1 \\
Ácido Laurico (C12:0) & $0-1$ \\
Ácidos grasos insaturados & $36-43$ \\
Ácido Oleico (C18:1) & $26.3-35$ \\
Ácido Palmitoleico (C16:1) & $0-4$ \\
Ácido Linoleico (C18:2) & $1.7-3$ \\
Ácido Linolénico & $0-1$ \\
Otros & 1.6 \\
Triacilgliceroles & $\geq 70$ \\
1(3) palmitoil-3(1)estearoil-2-oleoglicerol (POS) & 42.2 \\
1(3)-distearoil-2-oleoilglicerol (SOS) & 24.2 \\
1,3-dipalmitoil-2-oleoilglicerol (POP) & 21.8 \\
\hline
\end{tabular}

Fuente: Naik \& Kumar, 2014; Parra et al., 2017; Sirbu, Corno, Ullrich, \& Kuhnert, 2018

Estudios realizados en los últimos años han establecido que el perfil de ácidos grasos de la grasa de cacao está constituido por un 57 a 64\% de ácidos grasos saturados y 36 a 43\% de ácidos grasos insaturados. Los ácidos grasos saturados que constituyen la grasa del cacao son: ácido palmítico (C16:0), ácido esteárico (C18:0), ácido laurico (C12:0), ácido mirístico (C14:0) y ácido araquídico (C20:0); además los ácidos grasos insaturados presentes son; ácido oleico (C18:1), ácido palmitoleico (C16:1), ácido linoleico (C18:2) y ácido linolénico (C18:3); de los cuales el ácido palmítico (C18:0), ácido esteárico (C18:0), ácido oleico (C18:1) y ácido linoleico (C18:2) se encuentran en mayor cantidad y contribuyen con aproximadamente el $98 \%$ del total de ácidos grasos. Los triacilgliceroles de la grasa de cacao contienen en su estructura ácidos grasos saturados en las posiciones 1, 3 y ácido oleico en la posición 2 (Tabla 7) (Naik \& Kumar, 2014; Parra et al., 2017; Riaño et al., 2016; Servent et al., 2018; Torres- 
Moreno et al., 2015). En la Tabla 8, se presenta los contenidos de los principales ácidos grasos presentes en cacao de diferentes orígenes geográficos.

Tabla 8. Principales ácidos grasos en cacao de diferentes países

\begin{tabular}{lllll}
\hline & \multicolumn{4}{c}{ Ácido Graso (\%) } \\
\cline { 2 - 5 } \multicolumn{1}{c}{ País } & Acido Esteárico & Ácido Oleico & Ácido Palmítico & Ácido linoleico \\
\hline Costa de Marfil & 36.9 & $32.9-33$ & $25.8-26.6$ & $2.6-2.8$ \\
Ghana & $36.69-37.6$ & $32.7-32.99$ & $25.3-25.46$ & $2.51-2.8$ \\
Indonesia & $36.88-37.3$ & $33.06-34.3$ & $24.1-25.13$ & $2.5-2.7$ \\
Brasil & $33.3-33.8$ & $34.5-36.5$ & $25.1-27.9$ & $3.5-3.6$ \\
Ecuador & $34.62-36$ & $34.6-34.91$ & $25.2-25.6$ & $2.6-3.04$ \\
Malasia & $36-37.4$ & $33.5-34$ & $24.9-26$ & $2.6-3.0$ \\
\hline
\end{tabular}

Fuente: Jahurul et al., 2013

La composición bioquímica de la grasa de cacao depende de varios factores, como la variedad, la edad de la plantación, la maduración de los frutos y las condiciones ambientales. La identificación y cuantificación de ácidos grasos en la manteca de cacao es de gran interés para los laboratorios de investigación, control de calidad y procesamiento de productos a base de cacao, ya que su presencia y distribución posicional en las moléculas de Triacilgliceroles depende de sus propiedades físicas y térmicas (Torres-Moreno et al., 2015; Żyżelewicz et al., 2016).

Datos epidemiológicos han demostrado que el perfil de ácidos grasos en los alimentos tiene un impacto directo en la salud humana. Se ha probado que generalmente las grasas no saturadas tienen un efecto hipocolesterolémico, mientras que, las grasas saturadas tienden a elevar los niveles de colesterol total y LDL (Lipp \& Anklam, 1998; Quiñones et al., 2012; Torres-Moreno et al., 2015). De acuerdo con las recomendaciones de la Food and Nutrition Board, la dosis de ingesta dietética para una población sana está en el rango del 20 a 35\% del contenido total de energía; sin embargo, el contenido de ácidos grasos saturados (SFA) debe ser lo más bajo posible. Los ácidos grasos poliinsaturados (PUFA) deben representar un máximo del $10 \%$ del total de calorías y los ácidos grasos monoinsaturados (MUFA) deben ser los componentes principales (Gibson \& Newsham, 2018).

En este sentido, estudios recientes en el campo cardiovascular han demostrado que el consumo de chocolate negro (principal producto de transformación del cacao), podría 
proporcionar un efecto benéfico debido a las características de su composición química (Gómez-Juaristi et al., 2011; Gutiérrez Maydata, 2002; Predan et al., 2019).

\subsection{Situación actual de la calidad del cacao "Nacional" ecuatoriano}

En el Ecuador se encuentra sembrado más del 50\% del cacao "Fino y de Aroma" del mundo, lo cual lo convierte en el principal productor de este tipo de cacao. Actualmente, existen pocos materiales de cacao Nacional puro, predominando las plantaciones producto del cruzamiento natural de la variedad Nacional con materiales introducidos desde Venezuela y Trinidad; a los que se conoce como complejo de cacao Nacional x Trinitario (Afoakwa et al., 2008; Caligiani, Palla, Acquotti, Marseglia, \& Palla, 2014; W. Tezara et al., 2015).

Por sus características individuales distintivas de sabores y aromas diferentes, el cacao ecuatoriano es único, especial y reconocido con la denominación de sabor arriba. En muestras de cacao estudiadas a nivel mundial, estas propiedades dependen del origen genético y el tratamiento poscosecha. Sin embargo, en el cacao Arriba estás características organolépticas dependen de las diversas condiciones naturales del cultivo, favorecidas por la ubicación privilegiada del país en la mitad del mundo y su diversidad de microclimas. A pesar de esto, la composición química de los granos se mantiene poco conocido hasta la fecha, encontrándose una alta variabilidad en su composición (PROECUADOR, 2013). 


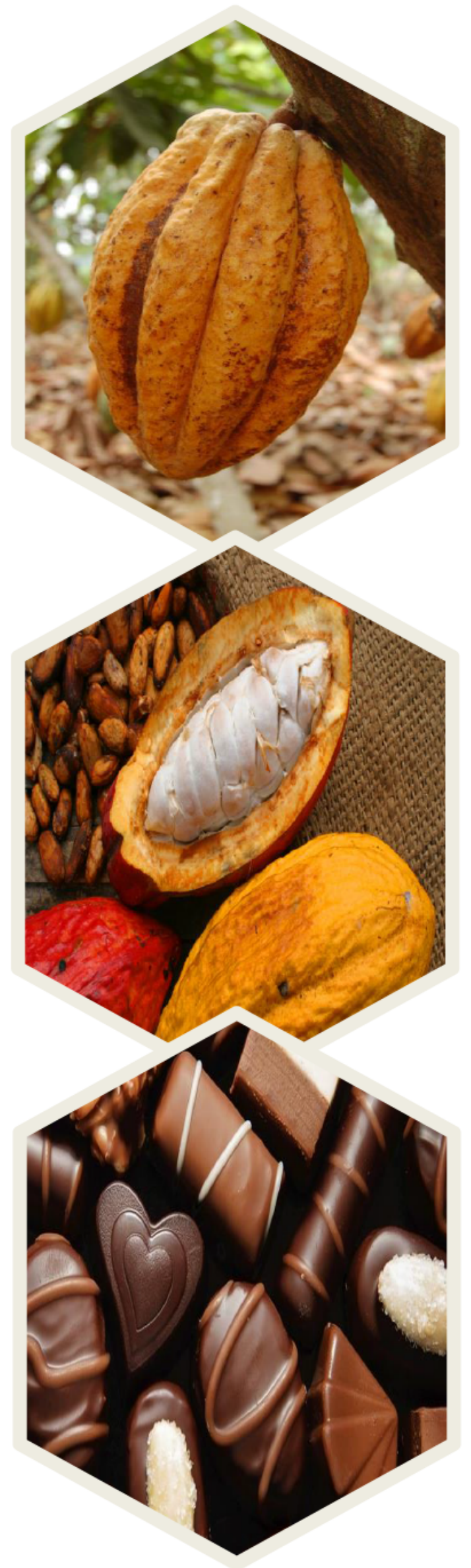

\section{Capítulo II.}

Objetivos del estudio 
En el Ecuador existe un tipo de cacao único en el mundo, identificado con el nombre de "Nacional"; el cual se reconoce por tener una fermentación muy corta y dar un chocolate suave de buen sabor y aroma, por lo que es catalogado con la clasificación de "Fino y de Aroma". Este tipo de cacao, tiene características individuales distintivas; con notas florales, frutales, nueces, almendras, especias que lo hacen único y especial.

Actualmente, en el Ecuador la calidad de los granos de cacao se evalúa mediante pruebas físicas (peso de las almendras, condición interna de los cotiledones, color y daños evidentes por infestaciones de hongos o insectos) y pruebas organolépticas que requieren de expertos y son de carácter subjetivo. La tendencia mundial en los últimos años, es caracterizar la composición química de las almendras de cacao; con la finalidad de establecer parámetros objetivos que permitan definir su calidad y certificar su origen.

En este contexto, el objetivo de esta investigación ha sido caracterizar los principales compuestos químicos no volátiles (metilxantinas, polifenoles y ácidos grasos) en almendras de cacao Nacional, como una herramienta de calidad organoléptica y nutricional para productores, exportadores e industriales de cacao de Ecuador.

En el estudio se plantearon los siguientes objetivos específicos:

1. Caracterizar el cacao Nacional Fino y de Aroma, a nivel de sus principales componentes químicos no volátiles.

2. Evaluar el efecto de la zona geográfica de producción, sobre el contenido de los principales componentes químicos (metilxantinas, polifenoles, grasa y perfil de ácidos grasos) del cacao ecuatoriano; como herramienta de calidad para productores, exportadores e industriales. 


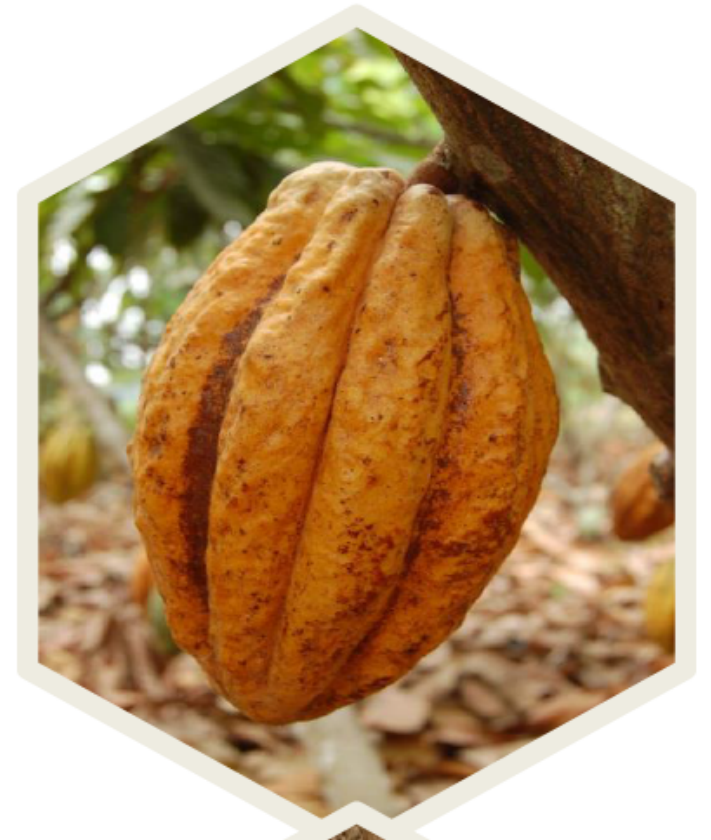

\section{Capítulo III.}

Publicaciones

generadas en la

presente Tesis

Doctoral 


\section{PUBLICACIÓN I}

Effect of the growing area on the methylxanthines and flavan-3-ols content in cocoa beans from Ecuador

Iván Samaniego, Susana Espín, James Quiroz,

Bladimir Ortiz, Wilman Carrillo, Cristina GarcíaViguera, Pedro Mena

Journal of Food Composition and Analysis 88 (2020) 103448

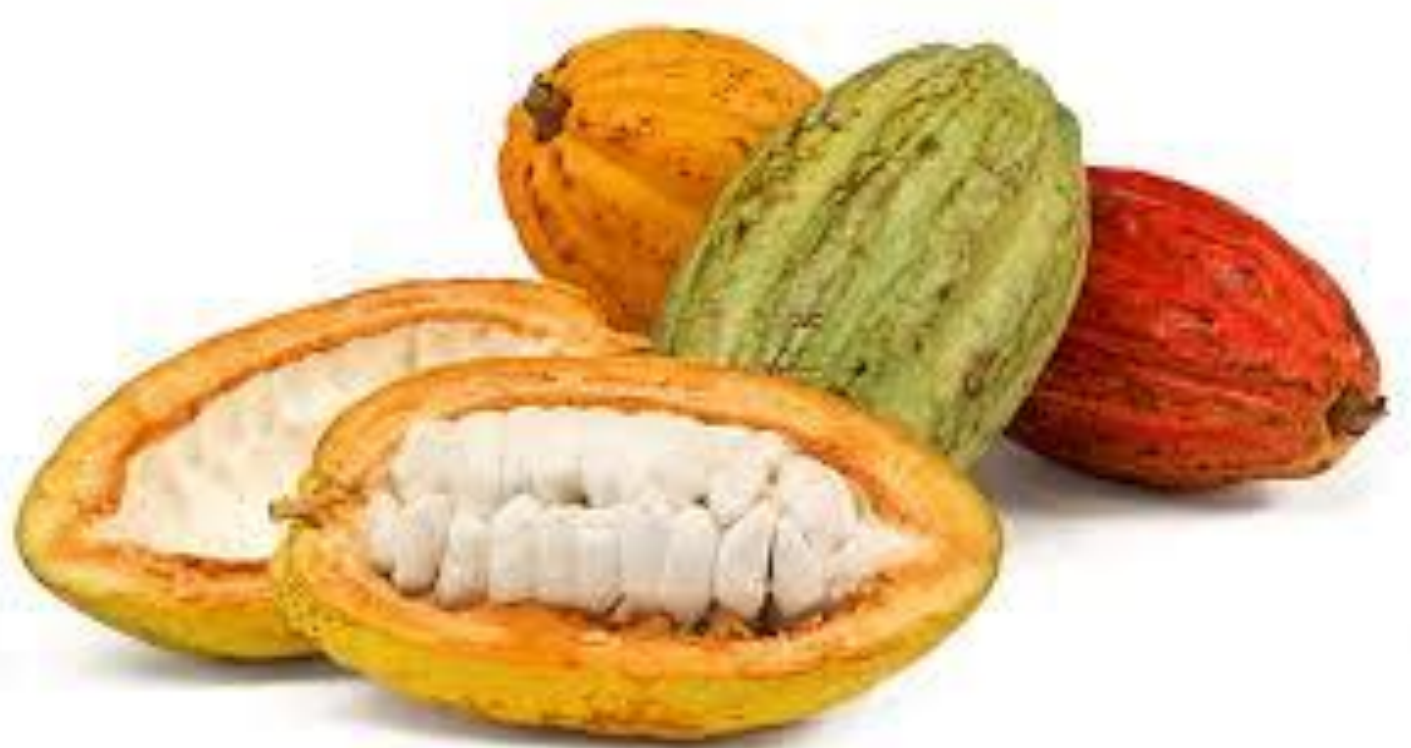




\section{Introduction}

Cocoa (Theobroma cacao L.), the main ingredient in chocolate, is one of the most important perennial crops by economic importance for developing countries in Africa, Latin America, and Asia. Cocoa traders distinguish traditionally four varieties: Criollo, Forastero, Trinitario (an hybrid of Criollo and Forastero), and the well-known Nacional from Ecuador (De Taeye, Bodart, Caullet, \& Collin, 2017). While most of the world cocoa production belongs to the variety Forastero ("bulk" grade) grown in Africa, Ecuador is one of the main exporters of cocoa in Latin America and the first producer of high-quality "fine" grade cocoa beans in the world (Afoakwa et al., 2008; Dand, 2011b; Exporters-Ecuador National Association of Cocoa, 2016; International Cocoa Organization, 2017; Kongor et al., 2016; Vargas Jentzsch et al., 2016). Nacional ("National") is a worldwide recognized cocoa variety produced in Ecuador and classified as "fine" grade, together with Criollo and Trinitario cocoas. It differs from other varieties by having a very short fermentation and yielding seeds of an excellent taste and aroma (Afoakwa et al., 2008, 2011; Dand, 2011a; Kongor et al., 2016; Motamayor et al., 2008; Oracz \& Nebesny, 2014). Nowadays, there are few materials of pure Nacional cocoa, being the predominant plantations natural hybrids between pure Nacional and Trinitario beans introduced from Venezuela and Trinidad.

This cacao is known as the Nacional x Trinitario complex and it is so-called "Arriba", whose distinctive individual organoleptic characteristics make unique, specific contributions to the flavor profile of chocolate products (Afoakwa et al., 2008; Caligiani et al., 2014; W. Tezara et al., 2015). The main components of cocoa are fat, carbohydrates, proteins, vitamins, minerals, and a range of secondary compounds biologically active for the human body, including methylxanthines (theobromine, and caffeine) and phenolic compounds (phenolic acids and flavonoids) (D'Souza et al., 2017; do Carmo et al., 2017; Wang, Lee, Lewis, Kamath, \& Archer, 1999). Cocoa is the largest natural source of TBR (around $3.7 \%$ in degreased sample) and has a low CAF content (0.2\% in degreased sample) (Acierno et al., 2016; Afoakwa et al., 2008; Araujo et al., 2014; Belščak et al., 2009). On the other hand, polyphenolic compounds 
participate in biochemical reactions contributing to the development of cocoa color and aroma, and they are responsible for sensory attributes of chocolate such as the presence of bitter and astringent notes (Saltini et al., 2013; Teye et al., 2015; Wollgast \& Anklam, 2000). The characterization of the phenolic content in beans is thus an useful tool to determine cocoa quality. Cocoa bean cotyledons are very rich in polyphenols, reaching up to 15 and $20 \%$ by weight in dried, degreased beans and $5 \%$ in fermented beans. Proanthocyanidins (58\%), flavan-3-ols monomers $(+)$ catechin, CAT, and (-) epicatechin, EPI, 37\%) and anthocyanins (4\%) are the three main groups of phenolics in cocoa beans (Araujo et al., 2014; Belščak et al., 2009; D'Souza et al., 2017; Hue et al., 2014; Nara Batista, Pereira de Andrade, Lacerda Ramos, Ribeiro Dias, \& Freitas Schwan, 2016; Saltini et al., 2013). This phytochemical profile, rich in methylxanthines and polyphenols, participates in the quality and potential health effects of cocoa and its derivative products (i.e., dark chocolate) (Hooper et al., 2012; Kerimi \& Williamson, 2015; Ludovici et al., 2017). Despite the importance of phenolic content to assess the quality of cocoa, an individual characterization of the main phenolic compounds is needed to fully understand the prospects of specific cocoas to produce high-quality chocolate products (Pereira-Caro et al., 2013). Flavan-3-ols monomers (catechins) and oligomers (proanthocyanidins) are the main cocoa phenolics and their contents depend on the producing area, among other factors (Żyżelewicz et al., 2016).

The quantity of cocoa bioactives depends, to a large extent, on intrinsic factors such as genotype and extrinsic factors such as agronomic management, soil conditions, climatic conditions, and post-harvest management (specially fermentation and bean drying) (Kongor et al., 2016). Studying the origin of cocoa is becoming of paramount importance to meet the demand for high-quality, rich in bioactives, and single-origin cocoa products (Marseglia et al., 2016). While the differences among cocoa-growing countries have been deeply evaluated (Caligiani et al., 2014; Cambrai, Marchioni, Julien-david, \& Marcic, 2017; Marseglia et al., 2016; Rodríguez-Carrasco, Gaspari, Graziani, Santini, \& Ritieni, 2018) little is known at a country level (Carrillo et al., 2014). In the case of Ecuadorian Nacional beans, the impact of ecological conditions on the bioactive composition of cocoa has not been assessed to date. However, there is a broad edaphoclimatic diversity among growing regions (for instance, Pacific coast and Amazonian area). These different conditions might lead to a notable variability in the phytochemical content of Nacional cocoa beans. Moreover, their phytochemical characterization represents a valuable quality tool for producers, exporters, and industrialists to support the competitive development of the Ecuadorian cocoa 
production chain and the certification of origin. The objective of this study was to determine the content of methylxanthines (TBR and CAF), total phenolic content, flavan-3-ols monomers (CAT and EPI), and major proanthocyanidins in beans of Nacional cocoa from the main producing areas of Ecuador.

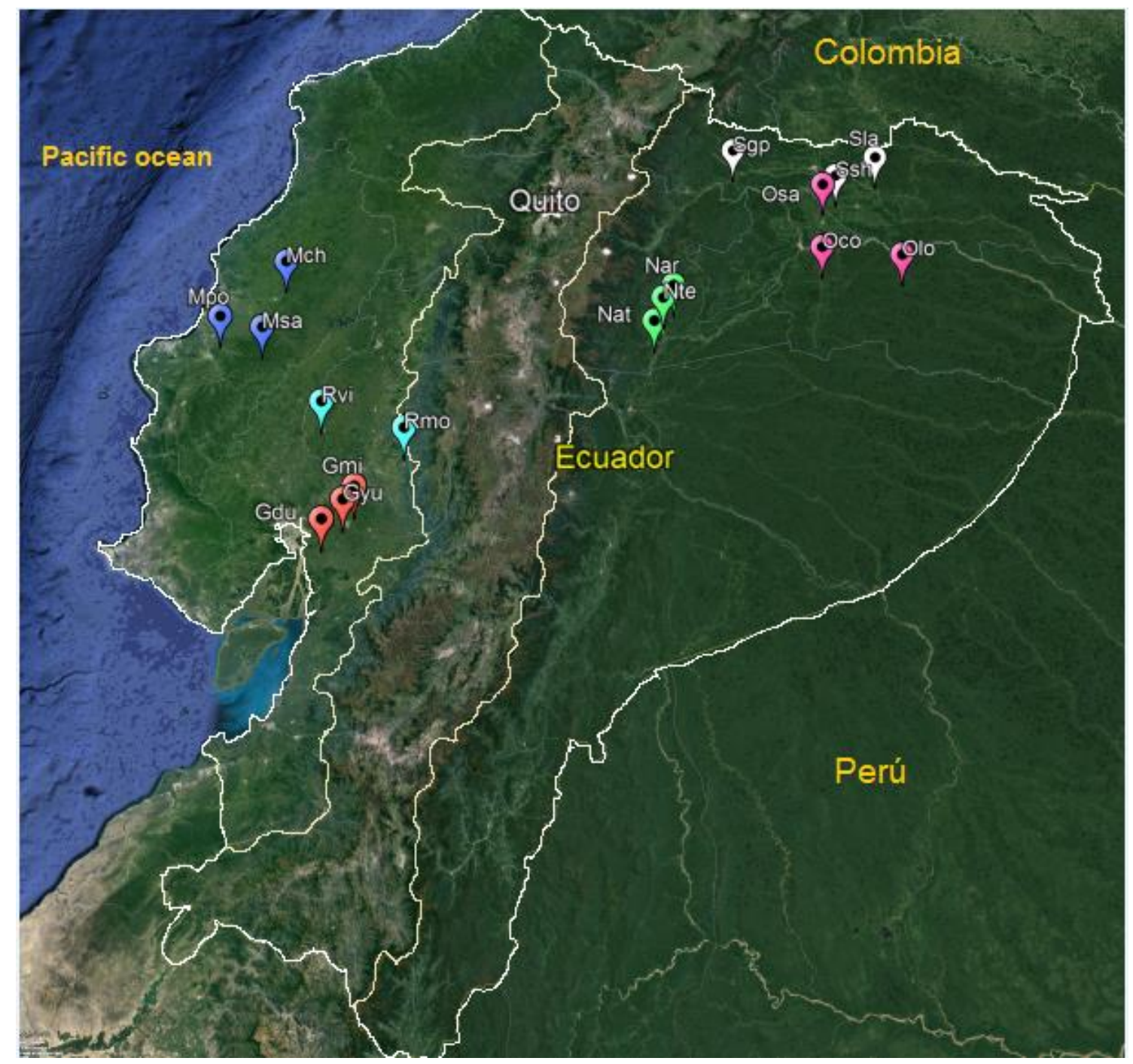

Figure.1. Geographical location of Ecuadorian cocoa-growing areas under study

\section{Material and methods}

\subsection{Plant material and sampling}

Samples of Nacional cocoa belonging to the Nacional $\mathrm{x}$ Trinitario complex (Arriba flavor) were taken directly from the trees in selected farms of Ecuador, representative from each growing area. The two main growing areas, Pacific Coast and Amazonian, were chosen. The cocoa-growing region of the Ecuadorian Pacific Coast is located from the west part of the Andes to the Pacific Ocean, while the Amazonian region is 
located from the east part of the Andes to the Peruvian and Colombian borders. Based on the Ecuadorian cocoa map, the three provinces with the highest production were selected for each region: in the Pacific Coast region, samples were taken in the provinces of Guayas, Los Ríos, and Manabí (accounting for $70 \%$ of the national production); in the Amazonian region, from the provinces of Sucumbíos, Francisco de Orellana, and Napo (6\% of production at national level) (Ministry of Agriculture and Livestock, 2016) (Figure 1). In each province, the three highest producing cantons were chosen and 5 representative farms within each canton were selected according to the lists of producers provided by local cocoa organizations (except for Los Ríos province, where only 2 samples were taken). A total of 17 cantons were selected (Table 1) and samples of Arriba flavor cocoa from 85 farms were collected.

Table 1. Ecuadorian cocoa-growing areas under study. Canton abbreviation in brackets

\begin{tabular}{|c|c|c|c|c|c|}
\hline N. & Region & Province & Canton & $\begin{array}{l}\text { Altitude } \\
\text { (m.a.s.l) }\end{array}$ & $\begin{array}{c}\text { Temperature, } \\
\text { annual } \\
\text { average } \\
\left({ }^{\circ} \mathbf{C}\right) \\
\end{array}$ \\
\hline 1 & & & Milagro (Gmi) & $25-27$ & $23-29$ \\
\hline 2 & & Guayas & Yaguachi (Gyu) & $13-31$ & $23-27$ \\
\hline 3 & & & Durán (Gdu) & $8-15$ & $25-32$ \\
\hline 4 & Pacific & & Montalvo (Rmo) & $133-148$ & $22-26$ \\
\hline 5 & Coast & Los Ríos & Vinces (Rvi) & $11-25$ & $24-34$ \\
\hline 6 & & & Santa Ana (Msa) & $61-182$ & $24-31$ \\
\hline 7 & & Manabí & Portoviejo (Mpo) & $25-42$ & $24-31$ \\
\hline 8 & & & Chone (Mch) & $24-30$ & $23-31$ \\
\hline 9 & & & El Coca (Oco) & $282-294$ & $18-28$ \\
\hline 10 & & $\begin{array}{c}\text { Francisco de } \\
\text { Orellana }\end{array}$ & Joya de los Sachas (Osa) & $275-290$ & $22-31$ \\
\hline 11 & & & Loreto (Olo) & $368-422$ & $19-29$ \\
\hline 12 & & & Lago Agrio (Sla) & $250-268$ & $21-30$ \\
\hline 13 & Amazonian & Sucumbíos & Shushufindi (Ssh) & $283-285$ & $25-32$ \\
\hline 14 & & & Gonzalo Pizarro (Sgp) & $385-615$ & $24-32$ \\
\hline 15 & & & Tena (Nte) & $300-500$ & $21-29$ \\
\hline 16 & & Napo & Arosemena Tola (Nat) & $542-735$ & $21-29$ \\
\hline 17 & & & Archidona (Nar) & $665-710$ & $21-29$ \\
\hline
\end{tabular}


In each farm, two samples made up of 20 cocoa fruits, randomly selected from 20 cacao trees ( 1 fruit/tree), were harvested at their optimal ripeness in order to obtain approximately $1 \mathrm{~kg}$ of fermented and dried cacao per sample. The harvest cacao fruits, of each sample, were placed in jute bags and transported to the Post-harvest Cocoa Treatment Center.

\subsection{Post-harvest treatment, fermentation, and drying}

The process of fermentation and drying of the collected samples was carried out in the Post-harvest Cocoa Treatment Center of the South Littoral Experimental Station of the INIAP (Ecuadorian National Institute of Agricultural Research, Yaguachi canton, province of Guayas), at an altitude of $17 \mathrm{~m}$ a.s.I., average temperature of $24.6{ }^{\circ} \mathrm{C}$, and relative humidity of $83 \%$.

Beans were extracted with the mucilage and a micro fermentation technique was applied in laurel wood boxes of $120 \times 120 \times 80 \mathrm{~cm}$ (height $\times$ width $\times$ depth), with a capacity of $120 \mathrm{~kg}$. Twelve samples of each were placed per box, with a mass removal at $48 \mathrm{~h}$, and a fermentation time of $96 \mathrm{~h}$ as required for Nacional cacao. Subsequently, a process of natural sun drying was carried out in cement slots until obtaining a bean humidity of approximately $7 \%$. The fermented and dried samples were placed in paper bags and transported to the LSAIA Food Analysis and Research Service Laboratory of the Santa Catalina Experimental Station of INIAP.

\subsection{Sample preparation}

A representative subsample of $250 \mathrm{~g}$ was taken from each sample for analysis. Then, using a scalpel, husks were manually removed, and beans were frozen using liquid nitrogen. These samples were subjected to a milling and sieving process (Ultra Centrifugal mill Resch ZM 200, Hann, Germany), until obtaining a particle size smaller than $1 \mathrm{~mm}$.

Ten grams of cocoa powder were placed in a cellulose extraction thimble (33 x $88 \mathrm{~mm})$ to be degreased. The fat was separated using a Soxhlet extraction process for $12 \mathrm{~h}$ with $200 \mathrm{~mL}$ of petroleum ether. Fat-free samples were then recovered and placed at room temperature for one hour to eliminate the solvent residue. The degreased cocoa was stored in hermetic bottles at refrigeration temperature $\left(5^{\circ} \mathrm{C}\right)$ for further analysis. 


\subsection{Chemical reagents}

All the analytical grade and HPLC grade solvents and reagents were obtained from Merck (Darmstadt, Germany). Deionized water was obtained with a Milli-Q Academic water purification system (Millipore, Sao Paulo, Brazil). The standards of CAT, EPI, procyanidins B2 (PC-B2) and C1 (PC-C1), CAF, and TBR were obtained from SigmaAldrich (St. Louis, Missouri, USA).

\subsection{Determination of methylxanthines by HPLC-DAD}

TBR and CAF determination was carried out in samples of $0.3 \mathrm{~g}$ of degreased cocoa sample with $90 \mathrm{~mL}$ of deionized water (Espín \& Samaniego, 2016). Samples were boiled for 30 min until the volume was reduced by half $(50 \mathrm{~mL})$. Then, $1 \mathrm{~mL}$ of Carrez 1 solution ( $15 \% \mathrm{w} / \mathrm{v}$ potassium hexacyanoferrate) and Carrez 2 (30\% w/v zinc sulfate) were added. The extract was immediately filtered through Whatman filter paper No. 4 in a volumetric balloon of $100 \mathrm{~mL}$. The extract was cooled and brought to volume with deionized water. An aliquot of the extract was passed through a Millipore PVDF membrane of $0.22 \mu \mathrm{m}$ and placed in a screw cap vial for analysis by High Performance Liquid Chromatography (HPLC).

Analyses were performed using an Agilent 1100/1200 Series HPLC (Waldbronn, Germany), consisting of a binary pump (G1312A), column furnace (G1316A), diode array detector (DAD, G1315D), auto-injector (G1329A), and controlled by the Chemstation software (Agilent Technologies, Waldbron, Germany). Separation was carried out using an Agilent Zorbax SB C18 column (150 mm x $4.6 \mathrm{~mm}, 5 \mu \mathrm{m}$ particle size), at a flow rate of $1 \mathrm{~mL} / \mathrm{min}$ and a temperature of $25^{\circ} \mathrm{C}$, using as mobile phase a $25 \%(\mathrm{v} / \mathrm{v})$ methanol solution in deionized water. For the analysis, $20 \mu \mathrm{L}$ of sample were injected into the equipment and methylxanthines were monitored using the DAD at 273 $\mathrm{nm}$. The identification and quantification of TBR and CAF was performed by comparison with their respective standards. Results were expressed as grams of compound per $100 \mathrm{~g}$ of degreased cocoa ( $\mathrm{g} / 100 \mathrm{~g}$ DW).

\subsection{Total phenolic content (TPC)}

TPC was assessed by UV-visible spectrophotometry, using an official method for the analysis of chemical parameters associated with the quality of cocoa (Espín \& Samaniego, 2016). This method is based on Cros \& Vincent, (1982). Briefly, one gram of degreased cocoa powder was weighed and subjected to a magnetic stirring 
extraction process with methanol/water $(70 / 30, \mathrm{v} / \mathrm{v})$ for $45 \mathrm{~min}$. Subsequently, the sample was filtered through Whatman No. 4 filter paper in a $100 \mathrm{~mL}$ volumetric flask and adjusted to volume with the extraction solution. One $\mathrm{mL}$ of the extract solution, 6 $\mathrm{mL}$ of deionized water, and $1 \mathrm{~mL}$ of Folin-Ciocalteu's reagent were mixed and left to rest for $3 \mathrm{~min}$. Then, $2 \mathrm{~mL}$ of $20 \%$ sodium carbonate solution (w/v) were added to the mix and heated to $40^{\circ} \mathrm{C}$ in a water bath for $2 \mathrm{~min}$. The absorbance was recorded at $760 \mathrm{~nm}$ in a Shimadzu model 2201 spectrophotometer (Shimazu, Kyoto, Japan). Quantification was performed using gallic acid as standard and the content of TPC was expressed as mg of gallic acid equivalents per gram of degreased cocoa (mg GAE/g DW).

\subsection{Determination of the content in main cocoa flavan-3-ols}

The analysis of the content of main flavan-3-ols (CAT, EPI, PC-B2, and PC-C1) in degreased cocoa powder was carried out using the method proposed by Hue et al. (2014), with minor modifications (Espín \& Samaniego, 2016). $0.3 \mathrm{~g}$ of degreased cocoa was weighed in a $15 \mathrm{~mL}$ centrifuge tube and $3 \mathrm{~mL}$ of acetone/water/formic acid solution (70/30/0.1, v/v/v) were added. The sample was subjected to an extraction process in a Mistral Multi-Mixer shaker (Melrose Park, USA) for $3 \mathrm{~min}$ and then in a Cole-Parmer model 8892 ultrasound bath (Chicago, USA) for $10 \mathrm{~min}$. Subsequently, the sample was centrifuged in a Damon/IEC division centrifuge (Needham Hts., MA, USA) for 10 min at $5500 \mathrm{rpm}(2706 \times \mathrm{g})$ at $5{ }^{\circ} \mathrm{C}$. The supernatant was separated and passed to a volumetric balloon of $25 \mathrm{~mL}$. This process was repeated 3 more times and the sample was brought to volume with the extraction solution. The extract was then filtered through a Millipore PVDF $0.22 \mu \mathrm{m}$ membrane and placed in a screw cap vial for further analysis.

Samples were analyzed by using the HPLC-DAD instrument before mentioned. Separation was performed on an Agilent Eclipse XDB C18 column (250 mm x $4.6 \mathrm{~mm}$, $5 \mu \mathrm{m}$ particle size), using as mobile phases an acetonitrile/water/formic acid solution $(99 / 0.8 / 0.2 \mathrm{v} / \mathrm{v} / \mathrm{v}$ ) (solvent $\mathrm{A}$ ) and acetonitrile (solvent $\mathrm{B}$ ) at a flow rate of $0.8 \mathrm{~mL} / \mathrm{min}$, with an elution gradient from $5 \% \mathrm{~B}$ to $100 \% \mathrm{~B}$ in $67 \mathrm{~min}$, at a temperature of $35{ }^{\circ} \mathrm{C}$. 20 $\mu \mathrm{L}$ of extract were injected into the equipment and flavan-3-ols were detected at 280 $\mathrm{nm}$. Identification and quantification were performed by comparison with the respective standards. Results were expressed in mg of flavan-3-ols per gram of degreased cocoa. 


\section{Statistical analysis}

Results are reported as mean \pm standard deviation $(n=5)$ of bioactive in degreased cocoa powder (DW). Significant statistical differences between data sets (cantons and provinces) were evaluated by one-way ANOVA. Tukey's multiple comparison test was carried out using Statistica 10.0 software for Windows (StatSoft, Paris, France). Principal Component Analysis (PCA) was determined using the correlation matrix and varimax analysis with Kaiser normalization; subsequent comparison of scores for principal components (PCs) was carried out by $t$-test or ANOVA with Tukey's multiple comparison test using the IBM SPSS Statistics 25 software package (SPSS Inc., Chicago, IL, USA).

\section{Results and discussion}

\subsection{Content of methylxanthines in Ecuadorian cocoa beans}

The TBR and CAF content per canton in degreased powders of Nacional fermented cocoa from 5 farms is shown in Table 2. As it has been previously reported by Brunetto et al. (2007), TBR was present in higher concentrations than CAF in the cocoa beans. TBR content was higher in the cantons from the Amazonian region than in the cantons of the Pacific Coast: values ranged between $2.08 \pm 0.14$ and $2.56 \pm 0.64 \mathrm{~g} / 100 \mathrm{~g} \mathrm{DW}$ for the Amazonian cantons and from $1.52 \pm 0.21$ to $1.85 \pm 0.35 \mathrm{~g} / 100 \mathrm{~g}$ DW for the Pacific Coast cantons. This trend was also observed for the CAF content (from $0.32 \pm$ 0.06 to $0.43 \pm 0.11$ and from $0.20 \pm 0.05$ to $0.33 \pm 0.09 \mathrm{~g} / 100 \mathrm{~g}$ DW for the Amazonian and Pacific Coast cantons, respectively). Significant differences $(P<0.05)$ occurred among both provinces and cantons for TBR and CAF. These results indicated that there is an effect of the production area on the content of methylxanthines, which may directly influence the quality attributes of cocoa beans (Belščak et al., 2009; Brunetto et al., 2007; Carrillo et al., 2014).

The methylxanthine content of cocoa beans, in particular the TBR/CAF ratio, has been used as a good marker to classify cocoa beans by their genotype, constituting also an important tool to certify the quality of beans from different geographical origins (Afoakwa et al., 2008; Brunetto et al., 2007; Diomande et al., 2015; Saltini et al., 2013). The TBR/CAF ratio allows the classification of Trinitario, Forastero, and Criollo cocoas and has been used to explore the origin of Venezuelan and Colombian cocoas Brunetto et al. (2007) and Carrillo et al. (2014). 
Table 2. Content of methylxanthines in Nacional cocoa from the main growing areas of Ecuador

\begin{tabular}{|c|c|c|c|c|c|c|}
\hline \multirow{2}{*}{$\frac{\text { No }}{1}$} & \multirow{2}{*}{$\begin{array}{l}\text { Province } \\
\text { Guayas }\end{array}$} & \multirow{2}{*}{$\frac{\text { Canton }}{\text { Milagro }}$} & \multicolumn{2}{|c|}{ TBR $(\mathrm{g} / \mathbf{1 0 0 \mathrm { g } )}$} & \multicolumn{2}{|c|}{ CAF $(g / 100 g)$} \\
\hline & & & $1.59 \pm 0.20$ & $C f$ & $0.24 \pm 0.07$ & B def \\
\hline 2 & (Pac. Coast) & Yaguachi & $1.65 \pm 0.33$ & $C f$ & $0.26 \pm 0.06$ & B cdef \\
\hline 3 & & Durán & $1.63 \pm 0.08$ & $\mathrm{Cf}$ & $0.22 \pm 0.04$ & B ef \\
\hline 4 & Los Ríos & Montalvo & $1.52 \pm 0.21$ & $C f$ & $0.20 \pm 0.05$ & $B f$ \\
\hline 5 & (Pac. Coast) & Vinces & $1.85 \pm 0.35$ & $\mathrm{C}$ def & $0.33 \pm 0.09$ & B abcd \\
\hline 6 & Manabí & Santa Ana & $1.61 \pm 0.07$ & $C f$ & $0.26 \pm 0.05$ & B cdef \\
\hline 7 & (Pac. Coast) & Portoviejo & $1.68 \pm 0.08$ & $C$ ef & $0.31 \pm 0.06$ & B bcde \\
\hline 8 & & Chone & $1.65 \pm 0.17$ & $\mathrm{Cf}$ & $0.30 \pm 0.05$ & B bcde \\
\hline 9 & $\begin{array}{c}\text { Francisco } \\
\text { de }\end{array}$ & El Coca & $2.07 \pm 0.14$ & B bcd & $0.43 \pm 0.11$ & $\mathrm{~A}$ a \\
\hline 10 & Orellana & Loreto & $2.04 \pm 0.15$ & B cde & $0.34 \pm 0.07$ & A abcd \\
\hline 11 & (Amazon) & Sachas & $2.19 \pm 0.19$ & B abcd & $0.33 \pm 0.03$ & A bcd \\
\hline 12 & Sucumbíos & Lago Agrio & $2.15 \pm 0.11$ & $A B$ bcd & $0.33 \pm 0.06$ & A abcd \\
\hline 13 & (Amazon) & Shushufindi & $2.56 \pm 0.64$ & $A B a$ & $0.39 \pm 0.10$ & $A a b$ \\
\hline 14 & & Gonzalo Pizarro & $2.08 \pm 0.23$ & $\mathrm{AB}$ bcd & $0.32 \pm 0.06$ & A bcde \\
\hline 15 & Napo & Tena & $2.18 \pm 0.09$ & A bcd & $0.38 \pm 0.03$ & $A a b$ \\
\hline 16 & (Amazon) & $\begin{array}{c}\text { Arosemena } \\
\text { Tola }\end{array}$ & $2.33 \pm 0.15$ & $A a b c$ & $0.36 \pm 0.04$ & $A a b c$ \\
\hline 17 & & Archidona & $2.43 \pm 0.18$ & $A a b$ & $0.37 \pm 0.05$ & $A a b$ \\
\hline
\end{tabular}

Results are expressed as mean $\pm \mathrm{SD}(n=5)$. Different uppercase letters indicate statistically significant differences $(P<0.05)$ among provinces; different lowercase letters indicate significant differences $(P<0.05)$ among cantons. TBR, theobromine; CAF, caffeine.

In Ecuador, there are few plantations of genetically pure Nacional cocoa but a mixture of natural hybrids with Trinitario clones (Nacional x Trinitario complex, Arriba flavor) (W. Tezara et al., 2015). To date, information on the TBR/CAF ratio of Ecuadorian cocoas is lacking despite its usefulness for classification purposes. In this work, the correlation between Ecuadorian cocoa samples from different geographical origins based on the TBR/CAF relationship was assessed (Fig. 2). The Ecuadorian Arriba cocoa presented a TBR/CAF ratio in the range of 5.20 to 7.86 , showing that all the samples belonged to the group of Trinitario cocoas, according to the ranges established by Davrieux, Assemat, Boulanger \& Cross (2003). The growing province had no effect on the $\mathrm{TBR} / \mathrm{CAF}$ ratio $(P>0.05)$, indicating that geographical differences do not affect this relationship. These results support the role of the TBR/CAF ratio in determining the 
distribution of cocoa samples by genotype according to the degree of hybridization, confirming the studies carried out by Pedan et al. (2018) and Carrillo et al. (2014).

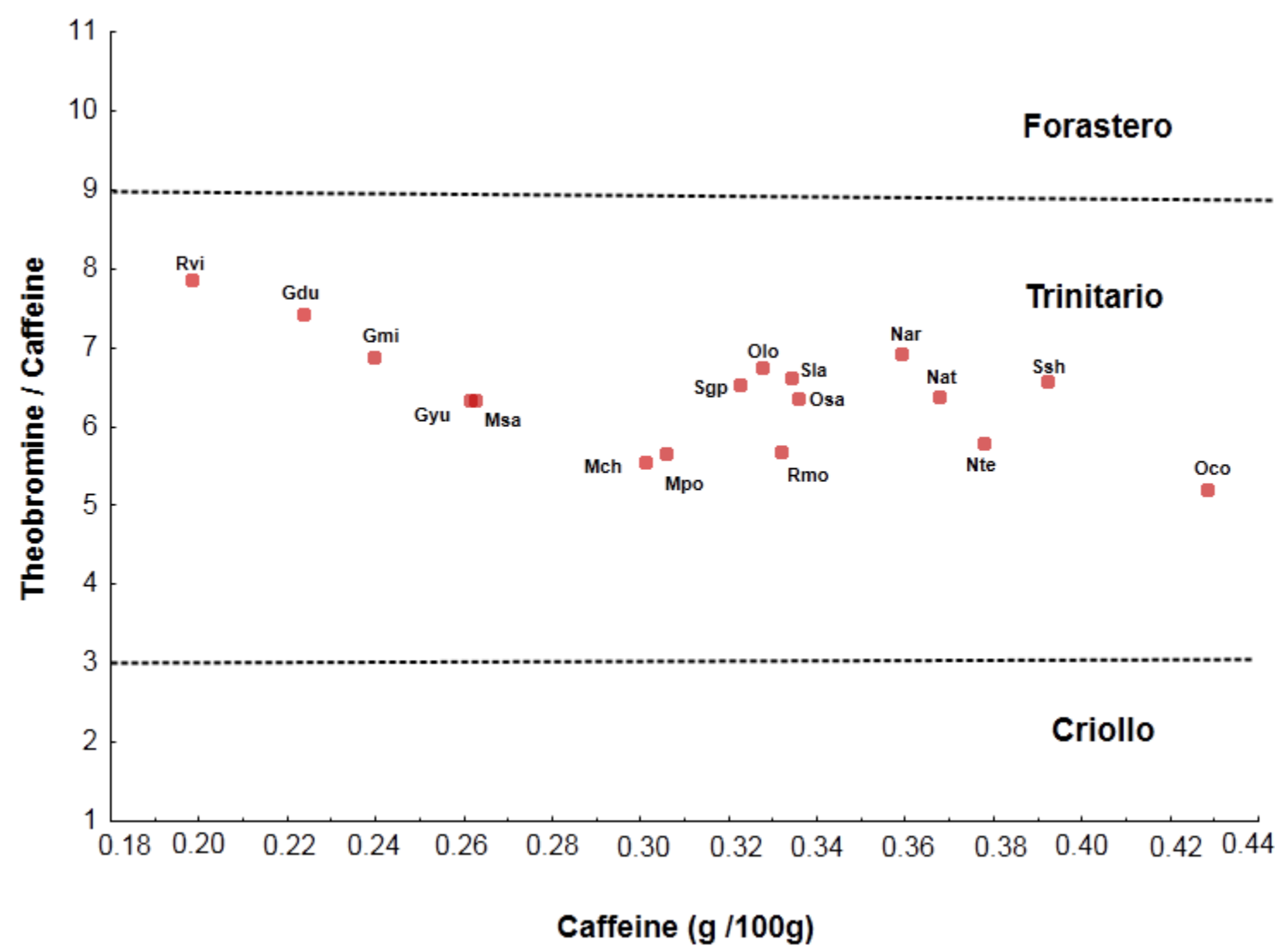

Figure 2. Relationship between the content of methylxanthines and the cocoa genotype. Sample codes are indicated at Table 1.

\subsection{Total phenolic content}

Table 3 shows the TPC of degreased Nacional cocoa in 17 cantons from both the Pacific Coast and Amazonian region. Data varied in the range of $33.55 \pm 5.74$ to 71.66 $\pm 3.94 \mathrm{mg} \mathrm{GAE} / \mathrm{g} \mathrm{DW}$. These results are within the ranges found by Wollgast \& Anklam (2000), who reported that the TPC content in fermented, dried, and fat-free Forastero cocoas usually varies between $5 \%$ to $10 \%(50 \mathrm{mg} / \mathrm{g}$ to $100 \mathrm{mg} / \mathrm{g} \mathrm{DW})$. The TPC content is lower, about $2 / 3$ of this concentration (33 to $66 \mathrm{mg} / \mathrm{g} \mathrm{DW}$ ), in Criollo cocoa beans. Similarly, these results are comparable to those achieved by other authors (Pedan et al., 2017), for 31 samples of fermented cocoa from different origins of Central and South America, who reported TPC values ranging from 32.76 to $61.13 \mathrm{mg}$ GAE/g DW. A study conducted by Jimenez, J., Amores, F., \& Solórzano (2014), on Ecuadorian Nacional cocoa samples collected in 6 different production areas showed that TPC varied between 34.67 and $100.05 \mathrm{mg}$ GAE/g DW. 


\section{Table 3. Flavan-3-ols content in Nacional cocoa from the main production areas of Ecuador}

\begin{tabular}{|c|c|c|c|c|c|c|c|c|c|c|c|}
\hline \multirow{2}{*}{$\begin{array}{c}\text { No } \\
1\end{array}$} & \multirow{2}{*}{$\begin{array}{c}\text { Province } \\
\text { Guayas }\end{array}$} & \multirow{2}{*}{$\begin{array}{l}\text { Canton } \\
\text { Milagro }\end{array}$} & \multicolumn{2}{|c|}{$\begin{array}{l}\text { CAT } \\
(\mathrm{mg} / \mathrm{g})\end{array}$} & \multicolumn{2}{|c|}{$\begin{array}{c}\text { EPI } \\
(\mathrm{mg} / \mathrm{g})\end{array}$} & \multicolumn{2}{|c|}{$\begin{array}{l}\text { PC-B2 } \\
(\mathrm{mg} / \mathrm{g})\end{array}$} & \multicolumn{2}{|c|}{$\begin{array}{l}\text { PC-C1 } \\
(\mathrm{mg} / \mathrm{g})\end{array}$} & $\begin{array}{c}\text { TPC } \\
\text { (mg GAE/g) }\end{array}$ \\
\hline & & & $4.82 \pm 1.34$ & C cde & $5.42 \pm 2.30$ & B cde & $3.19 \pm 1.16$ & B cdef & $2.76 \pm 0.67$ & $\mathrm{Cd}$ & $47.40 \pm 6.20 \quad b c$ \\
\hline 2 & (Pac. Coast) & Yaguachi & $4.81 \pm 1.16$ & C cde & $5.05 \pm 1.19$ & B de & $2.90 \pm 1.12$ & B def & $3.02 \pm 1.03$ & $\mathrm{Ccd}$ & $45.53 \pm 9.53 \mathrm{abc}$ \\
\hline 3 & & Durán & $4.20 \pm 0.86$ & $\mathrm{Ce}$ & $4.58 \pm 1.22$ & B de & $2.47 \pm 0.60$ & B def & $2.58 \pm 0.55$ & $\mathrm{Cd}$ & $45.51 \pm 7.60 \quad a b c$ \\
\hline 4 & Los Ríos & Montalvo & $6.03 \pm 1.83$ & BC bcde & $7.78 \pm 4.84$ & B bcd & $2.91 \pm 1.54$ & B def & $3.24 \pm 1.98$ & $\mathrm{BC} \mathrm{cd}$ & $43.45 \pm 8.56 a b c$ \\
\hline 5 & (Pac. Coast) & Vinces & $4.73 \pm 0.97$ & $\mathrm{BC}$ de & $4.96 \pm 1.53$ & B de & $2.13 \pm 0.50$ & B def & $2.56 \pm 0.81$ & $\mathrm{BC} d$ & $36.83 \pm 7.89 \quad a b$ \\
\hline 6 & Manabí & Santa Ana & $5.70 \pm 1.31$ & $A B$ bcde & $9.57 \pm 3.05$ & $\mathrm{~A} a b$ & $3.24 \pm 0.98$ & B cdef & $3.8 \pm 1.51$ & $\mathrm{~B} c \mathrm{~cd}$ & $33.55 \pm 5.74 \quad a$ \\
\hline 7 & (Pac. Coast) & Portoviejo & $8.93 \pm 4.94$ & $\mathrm{AB} a$ & $10.38 \pm 1.86$ & $A a b$ & $4.14 \pm 0.39$ & B bcd & $4.7 \pm 1.03$ & $\mathrm{~B} b \mathrm{bc}$ & $47.37 \pm 7.02 \quad b c$ \\
\hline 8 & & Chone & $6.27 \pm 1.37$ & AB abcde & $7.44 \pm 2.67$ & A bcd & $3.48 \pm 0.58$ & B bcdef & $3.67 \pm 1.13$ & $\mathrm{~B} c \mathrm{~d}$ & $46.80 \pm 8.81 b c$ \\
\hline 9 & Francisco & El Coca & $7.56 \pm 2.14$ & $\mathrm{AB}$ abcd & $5.5 \pm 1.63$ & B cde & $3.89 \pm 2.03$ & B bcde & $4.22 \pm 1.16$ & $\mathrm{BC}$ bcd & $49.39 \pm 4.82 \quad c$ \\
\hline 10 & de Orellana & Loreto & $5.84 \pm 1.25$ & $A B$ bcde & $3.52 \pm 1.27$ & $\mathrm{Be}$ & $1.77 \pm 0.53$ & $B$ ef & $2.53 \pm 0.91$ & $\mathrm{BC} d$ & $43.57 \pm 5.20 \quad a b c$ \\
\hline 11 & (Amazon) & Sachas & $7.08 \pm 1.05$ & AB abcde & $4.67 \pm 1.53$ & B de & $2.07 \pm 0.53$ & B def & $2.92 \pm 0.82$ & $\mathrm{BC} d$ & $50.39 \pm 2.55 \quad c$ \\
\hline 12 & Sucumbíos & Lago Agrio & $7.15 \pm 1.64$ & A abcd & $4.76 \pm 1.78$ & B de & $2.58 \pm 1.09$ & B def & $3.47 \pm 1.04$ & $\mathrm{~B} c \mathrm{~cd}$ & $52.86 \pm 12.40 \mathrm{~cd}$ \\
\hline 13 & (Amazon) & Shushufindi & $7.40 \pm 1.56$ & A abcd & $3.45 \pm 1.13$ & $\mathrm{Be}$ & $1.44 \pm 0.59$ & $B f$ & $2.55 \pm 0.84$ & $B d$ & $42.75 \pm 8.19 \quad a b c$ \\
\hline 14 & & Gonzalo Pizarro & $7.13 \pm 1.60$ & A abcd & $10.46 \pm 2.78$ & $\mathrm{~B} a b$ & $5.64 \pm 2.64$ & $\mathrm{~B}$ ab & $5.75 \pm 1.22$ & $\mathrm{~B} a b$ & $63.11 \pm 8.25$ de \\
\hline 15 & Napo & Tena & $7.38 \pm 0.82$ & A abcd & $5.31 \pm 1.19$ & A de & $2.84 \pm 1.25$ & A def & $3.11 \pm 0.74$ & $A c d$ & $48.50 \pm 5.19 b c$ \\
\hline 16 & (Amazon) & Arosemena Tola & $7.66 \pm 1.00$ & A abc & $8.94 \pm 2.21$ & $A b c$ & $5.17 \pm 1.80$ & $A b c$ & $5.83 \pm 1.28$ & $A a b$ & $71.66 \pm 3.94 \quad \mathrm{e}$ \\
\hline 17 & & Archidona & $7.74 \pm 2.56$ & $\mathrm{~A} a b$ & $13.16 \pm 3.61$ & $\mathrm{~A} \mathrm{a}$ & $7.85 \pm 3.29$ & $\mathrm{~A}$ a & $6.52 \pm 1.55$ & $\mathrm{~A} \mathrm{a}$ & $70.91 \pm 14.17 \mathrm{e}$ \\
\hline
\end{tabular}

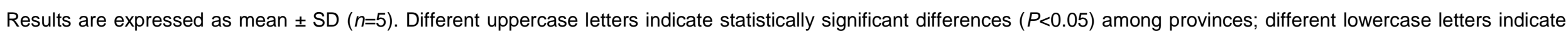
significant differences $(P<0.05)$ among cantons. CAT, $(+)$ catechin; EPI, $(-)$ epicatechin; PC-B2, procyanidin B2; PC-C1, procyanidin C1 and TPC, total phenol content. 
The upper limit values for these cocoas was higher than those obtained in this study. This variation between studies could rely on main factors affecting the phenolic content of cocoa beans, both intrinsic factors such as the genotype and extrinsic factors such as the harvest season and post-harvest processes (fermentation and drying) (Afoakwa et al., 2011; Oracz \& Nebesny, 2014; Wollgast \& Anklam, 2000).

It is known that geographical differences widely affect the cocoa beans TPC (Afoakwa et al., 2011; Oracz \& Nebesny, 2014; Wollgast \& Anklam, 2000). In this study, samples harvested in the Amazonian region cantons showed higher TPC than those obtained for Pacific Coast ones (between $42.75 \pm 8.19$ and $71.66 \pm 3.94 \mathrm{mg} \mathrm{GAE} / \mathrm{g}$ and between $33.55 \pm 5.74$ and $47.40 \pm 6.20 \mathrm{mg} \mathrm{GAE} / \mathrm{g}$, respectively). The average content of total phenolics contents per province varied in the following order: Napo > Sucumbíos > Francisco de Orellana > Guayas > Manabí > Los Ríos, being these differences among provinces statistically significant $(P<0.05)$. Similarly, differences at canton level for the TPC were also significant within the same province $(P<0.05)$, which denoted a high variation in the TPC of Ecuadorian cocoa based on the growing area. These results confirm previous studies by several authors (Carrillo et al., 2014; D'Souza et al., 2017; Othman et al., 2007; Pedan et al., 2018; Wollgast and Anklam, 2000), who also indicated that there is a significant effect of the production area on the TPC.

\subsection{Content of flavan-3-ols monomers and procyanidins}

Regarding flavan-3-ols monomers, the EPI content in these Nacional cocoa samples varied between $3.45 \pm 1.13$ and $13.16 \pm 3.61 \mathrm{mg} / \mathrm{g}$ DW (Table 3). These results were comparable to those reported by Wollgast \& Anklam (2000) for 10 samples of fermented cocoa beans from different origins in the world, with values ranging from 2.66 to $16.52 \mathrm{mg} / \mathrm{g}$ DW. Average global EPI content $(6.76 \mathrm{mg} / \mathrm{g}$ of degreased cocoa) was comparable to the ones obtained for Ecuadorian Arriba cocoa $(8.08 \mathrm{mg} / \mathrm{g}$ ) (Wollgast \& Anklam, 2000). Similarly, the present results showed that the EPI content in Ecuadorian Nacional cocoas grown in different geographical areas were within the range reported by Pedan et al. (2018), for cocoas harvested in several countries, this content varying between 1.55 and $17.58 \mathrm{mg} / \mathrm{g}$ DW. In the case of CAT, its content in Nacional cocoa samples ranged from $4.20 \pm 0.86$ to $8.93 \pm 4.94 \mathrm{mg} / \mathrm{g}$ DW (Table 3). Although EPI content is usually higher than the CAT content. Some studies are in agreement with our data (Oracz, Nebesny, \& Dorota, 2019; Rodríguez-Carrasco et al., 2018; Schinella et al., 2010). When geographical differences in the growing area were considered, significant statistical differences $(P<0.05)$ for the content of EPI and CAT 
were found for both provinces and cantons (Table 3). This accounted for an effect of the production area on the content of these flavan-3-ols monomers. In particular, samples from the Portoviejo, Gonzalo Pizarro, and Archidona cantons showed the highest contents of EPI $(10.38 \pm 1.86,10.46 \pm 2.78$, and $13.16 \pm 3.61 \mathrm{mg} / \mathrm{g} \mathrm{DW}$, respectively), while the samples from the Shushufindi and Loreto cantons presented the lowest EPI contents (3.45 \pm 1.13 and $3.52 \pm 1.27 \mathrm{mg} / \mathrm{g} \mathrm{DW}$, respectively) (Table 3). Regarding CAT, the highest values were found in the Archidona and Arosemena Tola cantons $(7.74 \pm 2.56$ and $7.66 \pm 1.00 \mathrm{mg} / \mathrm{g} \mathrm{DW}$, respectively) whereas the lowest values in the Durán and Vinces areas $(4.20 \pm 0.86$ and $4.73 \pm 0.97 \mathrm{mg} / \mathrm{g} \mathrm{DW}$, respectively). This effect of the cocoa origin on the CAT content was already confirmed by previous studies Pedan et al. (2018) and Caligiani et al. (2014).

The content of the main cocoa proanthocyanidins, dimer PC-B2 and trimer PC-C1, varied in the range of $1.44 \pm 0.59$ to $7.85 \pm 3.29$ and from $2.53 \pm 0.91$ to $6.52 \pm 1.55$ $\mathrm{mg} / \mathrm{g}$ DW, respectively. These results are slightly higher than those obtained by Pedan et al. (2018), for cocoa beans of different origins worldwide, showing PC-B2 and PC-C1 contents from 0.5 to $6.29 \mathrm{mg}$ and from 0.78 to $5.94 \mathrm{mg} / \mathrm{g} \mathrm{DW}$, respectively. On the contrary, the average content of PC-B2 and PC-C1 in the present study was $3.39 \mathrm{mg}$ and $3.72 \mathrm{mg} / \mathrm{g}$ DW, lower values than those reported by Wollgast \& Anklam (2000), (5.78 and $5.06 \mathrm{mg} / \mathrm{g}$ DW for dimeric and trimeric procyanidins, respectively, in cocoa samples with 96 hours of fermentation). Differences in the content of flavan-3-ols oligomers among studies could be due to the impact of fermentation, among other processes, on the flavan-3-ols content of cocoa (Diab, Hertz-Schünemann, Romy Streibel, \& Zimmer-Mann, 2014; Wollgast \& Anklam, 2000). The province and canton where the cocoa beans were grown altered significantly $(P<0.05)$ the content of PC-B2 and PC-C1 (Table 3). This finding evidenced the impact of the production area on the content of cocoa oligomeric procyanidins, which are partially responsible for the sensory profile of cocoa and are thus an important indicator of the quality of the beans (D'Souza et al., 2017; Pedan et al., 2018; Wollgast and Anklam, 2000).

\subsection{Multivariate analysis}

Principal component analysis (PCA) was applied to comprehensively assess the relationships among Nacional cocoa samples grown in different areas from Ecuador regarding their phytochemical composition. Although univariate analysis provided key insights on the effect of the growing area on the cocoa bioactive compounds, multivariate analysis served to better understand the impact of geographical 
differences on cocoa composition. Two PCs explained up to $80.0 \%$ of the total variance. The first PC (PC1) accounted for $46.5 \%$ of the total variability and had positive loadings from EPI, PC-B2, and PC-C1, as well as from TPC. The second PC (PC2), representing $33.5 \%$ of the total variance, was positively associated to CAF, TBR, and CAT (Fig. 3A).

A

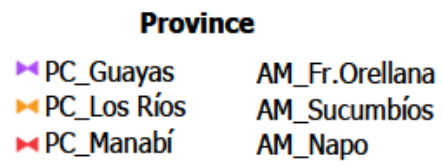

Figure 3. Principal component analysis (with varimax rotation) of the phytochemical composition of Nacional cocoa grown in the main Ecuadorian growing areas. A) loading plot of PC1 versus PC2; B) score plot and distribution of the samples in the consensus space. In the loading plot: TBR, theobromine; CAF, caffeine; TPC, total phenol content; EPI, (-)-epicatechin; CAT, (+) catechin; PC-B2, procyanidin B2; and PC-C1, procyanidin C1. In the score plot, circles correspond to cantons from the Amazonian region (AM), while double triangles account for cantons from the Pacific Coast (PC). Cantons from each province are abbreviated as reported in Table 1

Individual scores for each PC evidenced the effect of the growing area on the phytochemical composition of Ecuadorian Nacional cocoa (Fig. 3B). It is worth mentioning that $\mathrm{PC} 2$, related mainly to the methylxanthines composition of the beans, accounted for significant differences $(P<0.05)$ between the samples grown in the Pacific Coast and the Amazonian region. Cocoa samples from the Amazonian region showed positive PC2 scores, associated with higher TBR and CAF contents, while 
Pacific Coast were characterised by a lower methylxanthine content (negative PC2 scores) (Fig. 3B).

Table 4. Individual PC scores for each Nacional cocoa sample grown in Ecuador

\begin{tabular}{|c|c|c|c|c|c|c|c|c|c|c|}
\hline No & Province & Canton & & & PC1 & & & & PC2 & \\
\hline 1 & Guayas & Milagro & -0.15 & \pm & 0.49 & BC cdef & -1.01 & \pm & 0.68 & B def \\
\hline 2 & (Pac. Coast) & Yaguachi & -0.27 & \pm & 0.43 & BC def & -0.82 & \pm & 0.47 & B def \\
\hline 3 & & Durán & -0.40 & \pm & 0.31 & BC def & -1.10 & \pm & 0.25 & B ef \\
\hline 4 & Los Ríos & Montalvo & -0.16 & \pm & 1.05 & $\mathrm{BC}$ cdef & -0.15 & \pm & 0.90 & B bcdef \\
\hline 5 & (Pac. Coast) & Vinces & -0.49 & \pm & 0.39 & $B C$ def & -1.35 & \pm & 0.49 & $B f$ \\
\hline 6 & Manabí & Santa Ana & 0.23 & \pm & 0.70 & $A B$ bcde & -1.06 & \pm & 0.47 & B ef \\
\hline 7 & (Pac. Coast) & Portoviejo & 0.75 & \pm & 0.37 & $A B$ bcd & -0.25 & \pm & 1.01 & B bcdef \\
\hline 8 & & Chone & 0.15 & \pm & 0.65 & AB bcde & -0.53 & \pm & 0.69 & B cdef \\
\hline 9 & Francisco de Orellana & El Coca & -0.17 & \pm & 0.25 & C cdef & 0.97 & \pm & 0.79 & $A a b$ \\
\hline 10 & (Amazon) & Loreto & -0.99 & \pm & 0.49 & $\mathrm{C}$ ef & 0.33 & \pm & 0.56 & A abcd \\
\hline 11 & & Sachas & -0.68 & \pm & 0.32 & C ef & 0.61 & \pm & 0.24 & $A a b c$ \\
\hline 12 & Sucumbíos & Lago Agrio & -0.43 & \pm & 0.62 & BC def & 0.58 & \pm & 0.50 & $A a b c$ \\
\hline 13 & (Amazon) & Shushufindi & -1.32 & \pm & 0.40 & $B C f$ & 1.47 & \pm & 0.86 & A a \\
\hline 14 & & Gonzalo Pizarro & 1.32 & \pm & 0.51 & $\mathrm{BC} a b$ & 0.06 & \pm & 0.66 & A bcde \\
\hline 15 & Napo & Tena & -0.56 & \pm & 0.46 & A def & 0.87 & \pm & 0.24 & $A a b$ \\
\hline 16 & (Amazon) & Arosemena Tola & 1.08 & \pm & 0.52 & A $a b c$ & 0.83 & \pm & 0.14 & A $a b c$ \\
\hline 17 & & Archidona & 2.08 & \pm & 1.15 & $\mathrm{~A}$ a & 0.56 & \pm & 0.36 & $A a b c$ \\
\hline
\end{tabular}

Results are expressed as mean \pm SD $(n=5)$. Different uppercase letters indicate statistically significant differences $(P<0.05)$ among provinces; different lowercase letters indicate significant differences $(P<0.05)$ among cantons. PC, principal component; PC1, first principal component and PC2, second principal component.

Significant differences with respect to the methylxanthine content in the beans (PC2) were also observed at province and canton level $(P<0.05)$ for both geographical entities) (Table 4). While provinces grouped well for PC2 on the basis of the Pacific Coast and Amazonian regions, cantons showed a higher variability as some cantons belonging to the Pacific Coast region (i.e., Montalvo and Portoviejo) had similar PC2 scores to cantons in the Amazonian area (i.e., Gonzalo Pizarro and Loreto) (Table 4). Regarding PC1, linked with the content of EPI and procyanidins, the effect of 
geographical areas was not observed at region level $(P>0.05)$, but at a province and canton level ( $P<0.05$ for both geographical entities). Napo and Manabí were the provinces showed the highest flavan-3-ols content (positive PC1 scores), while Francisco de Orellana province presented the lowest flavan-3-ols content (negative PC1) (Table 4). Differences among cantons regarding PC1 accounted for the high variability on the flavan-3-ols content registered within each province (Table 4). This aspect was of note in the case of Sucumbíos province, where PC1 scores for Shushufindi and Gonzalo Pizarro cantons were different. In addition, although the three geographical levels considered (region, province, and canton) exhibited some common patterns, a high variability was observed among the 5 farms studied for some cantons (Fig. 3B). Nevertheless, despite data variability, some shared traits can be assigned to each Ecuadorian province. Los Ríos and Guayas provinces, from the Pacific Coast region, were characterised by a low content of cocoa bioactive. Manabí province, from the same region, showed higher flavan-3-ols values while it presented a low methylxanthine content in comparison to the Amazonian provinces. The Amazonian provinces Francisco de Orellana and Sucumbíos showed a high methylxanthine content while a reduced amount of flavan-3-ols. The Napo province, from the Amazonian region, displayed the highest content in cocoa bioactive. The individual characteristics of some provinces such as Manabí in the Pacific Coast and Napo in the Amazonian region could contribute to the development of the "designation of origin" concept for Ecuadorian cocoas from the Nacional variety, as it has been hypothesised for Colombian cocoas (Carrillo et al., 2014).

The differences in the content of bioactive in cocoa samples has been widely explored taking into account productions from different countries (Caligiani et al., 2014; Cambrai et al., 2017; Marseglia et al., 2016; Pedan et al., 2018). Caligiani et al. (2014), using also a PCA-based approach, described the phytochemical profile of Ecuadorian Arriba cocoas in comparison with Ghana, Trinidad, Grenada, Cameroon, and Ivory Coast samples from Criollo, Forastero, and Trinitario varieties. However, to date, little is known about intra-country differences. Carrillo et al. (2014), assessed the variability in the phytochemical composition of Colombian cocoas and observed a high variability depending on the growing area, but factors behind these differences were not fully unravelled. They indicated that the lower the altitude, the more flavan-3-ols are produced by the cocoa plant (Carrillo et al., 2014), in contrast to what has been reported for other plants (Criado, Morelló, Motilva, \& Romero, 2004). In the present work, the effect of altitude on the phenolic content was not observed, although it could 
be a factor behind the higher content of methylxanthines present in the Nacional cocoa samples grown in the Amazonian region (about $402 \mathrm{~m}$ a.s.l., while mean altitude for Pacific Coast region was $52 \mathrm{~m}$ a.s.I.). Nevertheless, other environmental factors related to the geographical origin of the farms assessed (such as precipitation, UV exposure, etc.) cannot be ruled out (Kongor et al., 2016; Oracz \& Nebesny, 2014).

\section{Conclusions}

Beans of Nacional cocoa cultivated in the main producing areas of Ecuador presented a high variability in the content of characteristic cocoa bioactives. The Amazonian region showed the highest methylxanthine content, cocoas from the Pacific Coast region had a lower methylxanthine content. The content of flavan-3-ols changed based on the province, without a clear effect on the growing region. Cantons were the geographical area having a major impact on the phytochemical content of Ecuadorian cocoas. A significant variability was observed among farms within the same canton. Despite this variability, common patterns were found for some provinces, which may be regarded as a possibility to further characterize the composition of cocoa beans for quality and authenticity purposes. Moreover, this study may help to identify the ideal soil and climatic conditions for the cultivation of cocoas looking for a specific profile of cocoa bioactive. Further studies assessing the final composition of cocoa-based products manufactured with different Ecuadorian cocoas are needed to fully understand the impact of the growing area on the final product.

\section{Acknowledgments}

The authors thank INIAP and SENECYT for funding this research through the Fortalecimiento and PIC-12-INIAP-003 projects, respectively.

\section{References}

Acierno, V., Yener, S., Alewijn, M., Biasioli, F., \& van Ruth, S. (2016). Factors contributing to the variation in the volatile composition of chocolate: Botanical, and geographical origin of the cocoa beans, and brand-related formulation and processing. Food Research International. https://doi.org/10.1016/j.foodres.2016.03.022

Adeyeye, E., \& Akinyeye, R. (2017). Effect of farm and industrial processing on the amino acid profile of cocoa beans. https://doi.org/10.1016/j.foodchem.2009.04.127 
Afoakwa, E. O., Paterson, A., Fowler, M., \& Ryan, A. (2008). Flavor formation and character in cocoa and chocolate: A critical review. https://doi.org/10.1080/10408390701719272

Afoakwa, E. O., Quao, J., Simpson Budu, A., Takrama, J., \& Kwesi Saalia, F. (2011). Effect of pulp preconditioning on acidification, proteolysis, sugars and free fatty acids concentration during fermentation of cocoa (Theobroma cacao) beans. https://doi.org/10.3109/09637486.2011.581224

Amores., F., Butler., D. ., Ramos., G., Sukha., D. A., Espín, S., Gomez., A., ... Seguine., E. (2007). Project to establish the physical, chemical and organoleptic parameters to differentiate between fine or flavor and bulk cocoa. Quevedo, Ecuador.

Aprotosoaie, A. C., Luca, S. V., \& Miron, A. (2016). Flavor Chemistry of Cocoa and Cocoa Products-An Overview. Comprehensive Reviews in Food Science and Food Safety, 15(1), 73-91. https://doi.org/10.1111/1541-4337.12180

Aprotosoaie, A. C., Luca, S. V., Miron, A., Arvelo Sánchez, M. A., González León, D., Maroto Arce, S., ... Batista, L. (2017). Flavor Chemistry of Cocoa and Cocoa Products-An Overview. In Santo Domingo, Republica Dominicana.Centro para el Desarrollo Agropecuario y Forestal CEDAF (Vol. 15). https://doi.org/10.1016/S0365-6691(10)70034-4

Araujo, Q., Fernandes, C., Ribeiro, D., Efraim, P., Steinmacher, D., Lieberei, R., ... Araujo, T. (2014). Cocoa Quality Index - a Proposal Quintino. Food Control. https://doi.org/10.1016/j.foodcont.2014.05.003

Asociación Nacional de Exportadores de Cacao. (2015). Historia del cacao. Retrieved June 3, 2020, from ANECACAO website: http://www.anecacao.com/es/quienessomos/historia-del-cacao.html

Balladares Grazzo, C. (2015). Caracterización físico - química de los lixiviados del cacao y café del litoral ecuatoriano, como potenciales fuentes de producción de bioetanol. UNIVERSIDAD DE LAS PALMAS DE GRAN CANARIA.

Batista, L. (2009). Guía Técnica El Cultivo de Cacao. In Santo Domingo, Republica Dominicana.Centro para el Desarrollo Agropecuario y Forestal CEDAF (Vol. 2). https://doi.org/10.1016/S0365-6691(10)70034-4

Belščak, A., Komes, D., Horžić, D., Ganić, K., \& Karlović, D. (2009). Comparative study of commercially available cocoa products in terms of their bioactive composition. 
Food Research International, 42, 707-716. https://doi.org/10.1016/j.foodres.2009.02.018

Brunetto, M. del R., Gutiérrez, L., Delgado, Y., Gallignani, M., Zambrano, A., Gómez, Á., ... Romero, C. (2007). Determination of theobromine, theophylline and caffeine in cocoa samples by a high- performance liquid ... Determination of theobromine, theophylline and caffeine in method with on-line sample cleanup in a switching-column system. Food Chemistry, 100(June), 459-467. https://doi.org/10.1016/j.foodchem.2005.10.007

Cabrera-Soto, L., Salinas-Moreno, Y., Velázquez-Cardelas, G., \& Espinosa-Trujillo, E. (2009). Contenido de fenoles solubles e insolubles en las estructuras del grano de maíz y su relación con propiedades físicas. Agrociencia, 43(8), 827-839.

Caligiani, A., Marseglia, A., Prandi, B., \& Palla, G. (2016). Pattern. Food Chemistry. https://doi.org/10.1016/j.foodchem.2016.05.072

Caligiani, A., Palla, L., Acquotti, D., Marseglia, A., \& Palla, G. (2014). Application of 1H NMR for the characterisation of cocoa beans of different geographical origins and fermentation levels. Food Chemistry, 157, 94-99. https://doi.org/10.1016/j.foodchem.2014.01.116

Cambrai, A., Marchioni, E., Julien-david, D., \& Marcic, C. (2017). Discrimination of Cocoa Bean Origin by Chocolate Polyphenol Chromatographic Analysis and Chemometrics. Food Analysis Methods, 10, 1991-2000. https://doi.org/10.1007/s12161-016-0744-7

Campos-Vega, R., Nieto-Figueroa, K. H., \& Oomah, B. D. (2018). Cocoa (Theobroma cacao L.) pod husk: Renewable source of bioactive compounds. Trends in Food Science and Technology, 81(September), 172-184. https://doi.org/10.1016/j.tifs.2018.09.022

CAOBISCO, ECA, \& FCC. (2015). Cocoa Beans: Chocolate and Cocoa Industry Quality Requirements.

Carrasco, O. (2015). Obtención de harina baja en gluten a partir de la cascarilla de cacao de las variedades CCN-51 y Nacional. UNIVERSIDAD TÉCNICA DE MACHALA.

Carrillo, L. C., Londoño-Londoño, J., \& Gil, A. (2014). Comparison of polyphenol, methylxanthines and antioxidant activity in Theobroma cacao beans from different cocoa-growing areas in Colombia. Food Research International, 60, 273-280. 
https://doi.org/10.1016/j.foodres.2013.06.019

Castro, M. C., Villagarcía, H., Nazar, A., Arbeláez, L. G., Massa, M. L., Del Zotto, H., ... Francini, F. (2020). Cacao extract enriched in polyphenols prevents endocrinemetabolic disturbances in a rat model of prediabetes triggered by a sucrose rich diet. Journal of Ethnopharmacology, 247(June 2019), 112263. https://doi.org/10.1016/j.jep.2019.112263

Colizzi, C. (2019). The protective effects of polyphenols on Alzheimer's disease: A systematic review. Alzheimer's and Dementia: Translational Research and Clinical Interventions, 5, 184-196. https://doi.org/10.1016/j.trci.2018.09.002

Criado, M. N., Morelló, J. R., Motilva, M. J., \& Romero, M. P. (2004). Effect of Growing Area on Pigment and Phenolic Fractions of Virgin Olive Oils of the Arbequina Variety in Spain. Journal of the American Oil Chemists' Society, 81(7), 633-640. https://doi.org/10.1007/s11746-004-954-z

Cros, E., \& Vincent, J. (1982). Recherche d'un indice de fermentation du cacao, 1: Evolution des tanins et des phénols totaux de la feve. Café, Cacao, Thé (Francia), 26(2), 109-114.

D’Souza, R., Grimbs, S., Behrends, B., Bernaert, H., Ullrich, M. S., \& Kuhnert, N. (2017). Origin-based polyphenolic fi ngerprinting of Theobroma cacao in unfermented and fermented beans. Food Research International, 99(March), 550559. https://doi.org/10.1016/j.foodres.2017.06.007

Dand, R. (2011a). Agronomics of international cocoa production. In The International Cocoa Trade (pp. 23-64). https://doi.org/10.1016/B978-0-85709-125-3.50002-1

Dand, R. (2011b). History and origins of the international cocoa trade. In The International Cocoa Trade (Third edit, pp. 1-22). https://doi.org/10.1016/B978-085709-125-3.50001-X

Davrieux, F., Assemat, S., Boulanger, R., \& Cros, E. (2003). Determination of cocoa purine content by near infrared spectroscopy [Poster]. In: 14th International Cocoa Research Conference, 13-18 October 2003, Accra, Ghana. - Montpellier: Cirad, 2003, 1 p.

de Souza, P. A., Moreira, L. F., Sarmento, D. H. A., \& da Costa, F. B. (2018). CacaoTheobroma cacao. Exotic Fruits, 3(2001), 69-76. https://doi.org/10.1016/b978-012-803138-4.00010-1

De Taeye, C., Bodart, M., Caullet, G., \& Collin, S. (2017). Roasting conditions for 
preserving cocoa flavan-3-ol monomers and oligomers: interesting behaviour of Criollo clones. Journal of the Science of Food and Agriculture, 97(12), 4001-4008. https://doi.org/10.1002/jsfa.8265

Diab, J., Hertz-Schünemann, Romy Streibel, T., \& Zimmer-Mann, R. (2014). On-line measurement of volatile organic compounds released during roasting ofcocoa beans. Food Research International, 63, 344-352. https://doi.org/10.1016/j.foodres.2014.04.047

Diomande, D., Antheaume, I., Leroux, M., Lalande, J., Balayssac, S., Remaud, G. S., \& Tea, I. (2015). Multi-element, multi-compound isotope profiling as a means to distinguish the geographical and varietal origin of fermented cocoa ( Theobroma cacao L .) beans. Food Chemistry, 188, 576-582. https://doi.org/10.1016/j.foodchem.2015.05.040

do Carmo, B., Campos, R., da Silva, R., Abreu, M. B., \& Lopes, A. (2017). Bioactive amines and phenolic compounds in cocoa beans are affected by fermentation. Food Chemistry, 228, 484-490. https://doi.org/10.1016/j.foodchem.2017.02.004

EL Telégrafo. (2019). Exportaciones de cacao subieron 4,65\% en 2018. Diario El Telégrafo.

Enríquez, G. (1985). Curso sobre el cultivo del cacao (Centro Agr). Retrieved from https://books.google.es/books?hl=es\&lr=\&id=eZgOAQAAIAAJ\&oi=fnd\&pg=PA5\&d q=enríquez+1985+cacao\&ots=lqxK0-Vm7N\&sig=0V-Psdo9kFZ6heOCCugnlTh$3 \mid c \# v=0 n e p a g e \& q=e n r i ́ q u e z 1985$ cacao\&f=false

Espín, S., \& Samaniego, I. (2016). Manual para análisis de parámetros químicos asociados a la calidad del cacao. In Manual Nro 105. Retrieved from http://181.112.143.123/bitstream/41000/2827/1/iniapsc322est.pdf

Espín, S., Samaniego, I., Wakao, H., \& Juan, J. (2007). La relación teobromina/cafeina asociada a la calidad del cacao ecuatoriano. Alimentos Ciencia e Ingeniería, 16(2), 107-109.

Exporters-Ecuador National Association of Cocoa. (2016). Sabor Arriba. Guayaquil, Ecuador.

Fernández, V., Yee, A., Sulbarán, B., \& Peña, J. (2014). Actividad antioxidante y contenido de polifenoles en chocolates comerciales venezolanos. Revista de La Facultad de Agronomia, 31(1), 129-144.

Franco-Agurto, G., \& Suárez-Quirumbay, K. (2014). Determinación del contenido de 
polifenoles y actividad antioxidante de una bebida láctea elaborada a base de residuos agroindustriales de cacao, café y naranja. Escuela Superior Politécnica del Litoral.

García-Vidal, G., Guzmán-Vilar, L., \& Campdesuñer, R. P. (2017). Research Trends in Cocoa: Opportunities for Research in Santo. SATHIRI, Sembrador, 12(2), 22.

Gibson, M., \& Newsham, P. (2018). Chocolate/Cacao. Food Science and the Culinary Arts, 341-352. https://doi.org/10.1016/b978-0-12-811816-0.00017-8

Gómez-Juaristi, M., González-Torres, L., Bravo, L., Vaquero, M. P., Bastida, S., \& Sánchez-Muniz, F. J. (2011). Efectos beneficiosos del chocolate en la salud cardiovascular. Nutricion Hospitalaria, 26(2), 289-292. https://doi.org/10.3305/nh.2011.26.2.5016

Grimbs, S., \& Ullrich, M. S. (2017). Origin-based polyphenolic fingerprinting of Theobroma cacao in unfermented and fermented beans. Food Research International, (June), 0-1. https://doi.org/10.1016/j.foodres.2017.06.007

Guanga, S. (2018). Estudio y aprovechamiento de los residuos del cacao de la compañía Nestlé como estrategia comercial. Universidad De Guayaquil.

Gutiérrez Maydata, B. A. (2002). Chocolate, polifenoles y protección a la salud. Acta Farmaceutica Bonaerense, 21(2), 149-152.

Hartel, R. W., von Elbe, J. H., \& Hofberger, R. (2018). Confectionery Science and Technology. In Confectionery Science and Technology. https://doi.org/10.1007/978-3-319-61742-8

Hooper, L., Kay, C., Abdelhamid, A., Kroon, P. A., Cohn, J. S., Rimm, E. B., \& Cassidy, A. (2012). Effects of chocolate, cocoa, and flavan-3-ols on cardiovascular health: a systematic review and meta-analysis of randomized trials $1-3$. American Journal of Clinical Nutrition, 95, 740-751. https://doi.org/10.3945/ajcn.111.023457.INTRODUCTION

Hue, C., Brat, P., Gunata, Z., Samaniego, I., Servent, A., Morel, G., ... Davrieux, F. (2014). Near infra-red characterization of changes in flavan-3-ol derivatives in cocoa (Theobroma cacao L.) As a function of fermentation temperature. Journal of Agricultural and Food Chemistry, 62(41), 10136-10142. https://doi.org/10.1021/jf501070d

Instituto Nacional Ecuatoriano de Normalización. Granos de cacao. Requisitos. , (2018). 
International Cocoa Organization. (2017). Annual Report 2014/2015.

Jahurul, M. H. A., Zaidul, I. S. M., Norulaini, N. A. N., Sahena, F., Jinap, S., Azmir, J., ... Mohd Omar, A. K. (2013). Cocoa butter fats and possibilities of substitution in food products concerning cocoa varieties, alternative sources, extraction methods, composition, and characteristics. Journal of Food Engineering, 117(4), 467-476. https://doi.org/10.1016/j.jfoodeng.2012.09.024

Jimenez, J., Amores, F., \& Solórzano, E. (2014). Componentes de identidad para reconocer las diferencias del cacao que se produce en varias regiones del Ecuador. Quevedo, Ecuador.

Jimenez, J., Amores, F., \& Solórzano, E., Jiménez, J., Amores, F., \& Solórzano, E. (2014). Componentes de identidad para reconocer las diferencias del cacao que se produce en varias regiones del Ecuador. Estación Experimental Tropical Pichilingue. INIAP. Boletín Técnico: 164. In Estación Experimental Tropical Pichilingue INIAP. (Vol. 164). Quevedo, Ecuador, Ecuador.

Jiménez, J., Amores, F., Nicklin, C., Rodríguez, D., Zambrano, F., Bolaños, M., ... Cedeño, P. (2011). Micro fermentación y análisis sensorial para la selección de árboles superiores de cacao. Retrieved from file://C:/Users/Owner/Downloads/Boletín técnico No 140 (2).PDF

Jovellanos-Fernández, E. (2016). Estudio del contenido de compuestos bioactivos del cacao y su aplicación en la obtención de un ingrediente rico en polifenoles para el diseño de un chocolate enriquecido (Universidad de Murcia). https://doi.org/10.17161/bi.v1i0.3

Jovellanos Fernandez, E. (2016). Estudio del contenido de compuestos bioactivos del cacao y su aplicación en la obtención de un ingrediente rico en (poli)fenoles para el diseño de un chocolate enriquecido (UNIVERSIDAD DE MURCIA). https://doi.org/10.17161/bi.v1i0.3

Kerimi, A., \& Williamson, G. (2015). The cardiovascular benefits of dark chocolate. Vascular Pharmacology, 71, 11-15. https://doi.org/10.1016/j.vph.2015.05.011

Kim, J., Lee, K. W., \& Lee, H. J. (2011). Cocoa (Theobroma cacao) Seeds and Phytochemicals in Human Health. In Nuts and Seeds in Health and Disease Prevention (pp. 351-360). https://doi.org/10.1016/B978-0-12-375688-6.10042-8

Konger Edem, J., Hinneh, M., Van de Walle, D., Afoakwa, E. O., Boeckx, P., \& Dewettinck, K. (2016). Factors influencing quality variation in cocoa (Theobroma 
cacao) bean flavour profile - A review. Food Research International, 82, 44-52. https://doi.org/10.1016/j.foodres.2016.01.012

Kongor, J. E., Hinneh, M., de Walle, D. Van, Afoakwa, E. O., Boeckx, P., \& Dewettinck, K. (2016). Factors influencing quality variation in cocoa ( Theobroma cacao ) bean flavour profile - A review. Food Research International, 82(January), 44-52. https://doi.org/10.1016/j.foodres.2016.01.012

Krähmer, A., Engel, A., Kadow, D., Ali, N., Umaharan, P., Kroh, L. W., \& Schulz, H. (2015). Fast and neat - Determination of biochemical quality parameters in cocoa using near infrared spectroscopy. 181, 152-159. https://doi.org/10.1016/j.foodchem.2015.02.084

Lefeber, T., Papalexandratou, Z., Gobert, W., Camu, N., \& Vuyst, L. De. (2012). Onfarm implementation of a starter culture for improved cocoa bean fermentation and its in $\mathrm{fl}$ uence on the $\mathrm{fl}$ avour of chocolates produced thereof. Food Microbiology, 30(2), 379-392. https://doi.org/10.1016/j.fm.2011.12.021

Lim, T. K. (2012). Edible Medicinal and Non Medicinal Plants Fruits. In Edible Medicinal and Non Medicinal Plants: Volume 3, Fruits (Vol. 3, pp. 1-159). https://doi.org/10.1007/978-94-007-2534-8

Lipp, M., \& Anklam, E. (1998). Review of cocoa butter and alternative fats for use in chocolate. Food Chemistry, 62(1), 73-97. https://doi.org/10.1016/S03088146(97)00160-X

Loor, R. G., Risterucci, A. M., Courtois, B., Fouet, O., Jeanneau, M., Rosenquist, E., ... Lanaud, C. (2009). Tracing the native ancestors of the modern Theobroma cacao L. population in Ecuador. Tree Genetics and Genomes, 5(3), 421-433. https://doi.org/10.1007/s11295-008-0196-3

López-Guerrero, A. (2017). Producción y comercialización de cacao Fino de Aroma en el Ecuador - Año 2012-2014". In Superintendencia de Control del Poder del Mercado (Vol. 102). https://doi.org/10.1002/ejsp.2570

Loureiro, G. A. H. A., Araujo, Q. R., Sodré, G. A., Valle, R. R., Souza, J. O., Ramos, E. M. L. S., ... Grierson, P. F. (2017). Cacao quality: Highlighting selected attributes. Food Reviews International, 33(4), 382-405. https://doi.org/10.1080/87559129.2016.1175011

Ludovici, V., Barthelmes, J., Nägele, M., Enseleit, F., Ferri, C., Flammer, A., ... Sudano, I. (2017). Cocoa, Blood Pressure, and vascular Function. 4(August), 1- 
12. https://doi.org/10.3389/fnut.2017.00036

Marseglia, A., Acquotti, D., Consonni, R., Cagliani, L. R., Palla, G., \& Caligiani, A. (2016). HR MAS $1 \mathrm{H} \mathrm{NMR}$ and chemometrics as useful tool to assess the geographical origin of cocoa beans - Comparison with HR $1 \mathrm{H}$ NMR. Food Research International Journal, 85, 273-281. https://doi.org/10.1016/j.foodres.2016.05.001

Martín, M. A., \& Ramos, S. (2016). Cocoa polyphenols in oxidative stress: Potential health implications. Journal of Functional Foods, 27, 570-588. https://doi.org/10.1016/j.jff.2016.10.008

Martínez, R., Torres, P., Meneses, M. A., Figueroa, J. G., Pérez-Álvarez, J. A., \& Viuda-Martos, M. (2012). Chemical, technological and in vitro antioxidant properties of cocoa (Theobroma cacao L.) co-products. Food Research International, 49(1), 39-45. https://doi.org/10.1016/j.foodres.2012.08.005

Mata Anchundia, D., Suatunces Cuñumai, J., Munoz Macías, S., Bayas, A., \& Herrera Soler, M. (2018). Dimensión climática en el comercio justo para el cacao fino de aroma (Theobroma cacao I.). Revista de Estudios Empresariales. Segunda Época, 2(2), 86-102. https://doi.org/10.17561/ree.v2018n2.5

Ministry of Agriculture and Livestock. (2016). Reportes Dinámicos ESPAC. In Superficie, Producción y Rendimiento (2002-2016) (Vol. 2017). Quito, Ecuador.

Mondino, C., \& Ferrato, J. (2006). El análisis sensorial, una herramienta para la evaluación de la calidad desde el consumidor. Agromensajes, 16-48.

Motamayor, J. C., Lachenaud, P., Wallace da Silva, J., Loor, R., Kuhn, D., Brown, S., \& Schnell, R. (2008). Geographic and genetic population differentiation of the Amazonian chocolate tree ( Theobroma cacao L ) Geographic and Genetic Population Differentiation of the Amazonian Chocolate Tree ( Theobroma cacao $\mathrm{L}$ ). Plos One, 3(10). https://doi.org/10.1371/journal.pone.0003311

Mundaca Vidarte, G. A. (2016). Análisis de la calidad del grano de cacao mediante imágenes hiperespectrales usando técnicas de visión artificial. UNVERSIDAD DE PIURA.

Muñoz Hernández, G. C. (2018). Evaluación de la capacidad antioxidante en cacao Nacional fino de aroma (Theobroma cacao L.), de las principales zonas productoras del Ecuador. UNIVERSIDAD CENTRAL DEL ECUADOR.

Naik, B., \& Kumar, V. (2014). Cocoa Butter and Its Alternatives: A Reveiw. Journal of 
Bioresource Engineering and Technology, 1(March 2014), 7-17.

Nara Batista, N., Pereira de Andrade, D., Lacerda Ramos, C., Ribeiro Dias, D., \& Freitas Schwan, R. (2016). Antioxidant capacity of cocoa beans and chocolate assessed by FTIR. Food Research International Journal, 1-7. https://doi.org/10.1016/j.foodres.2016.10.028

Ooi, T. S., Ting, A. S. Y., \& Siow, L. F. (2020). Influence of selected native yeast starter cultures on the antioxidant activities, fermentation index and total soluble solids of Malaysia cocoa beans: A simulation study. Lwt, 122(July 2019), 108977. https://doi.org/10.1016/j.lwt.2019.108977

Ooi, T. S., Ting, A. S. Y., Siow, L. F., Romanens, E., Freimüller Leischtfeld, S., Volland, A., ... Tavares Bisneto, J. D. (2020). Screening of lactic acid bacteria and yeast strains to select adapted anti-fungal co-cultures for cocoa bean fermentation. $\begin{array}{llll}\text { Food Chemistry, 290(October } & \text { 2019), }\end{array}$ https://doi.org/10.1016/j.Iwt.2019.108977

Oracz, J., \& Nebesny, E. (2014). Influence of roasting conditions on the biogenic amine content in cocoa beans of different Theobroma cacao cultivars. Food Research International, 55, 1-10. https://doi.org/10.1016/j.foodres.2013.10.032

Oracz, J., Nebesny, E., \& Dorota, Ż. (2019). Identification and quantification of free and bound phenolic compounds contained in the high-molecular weight melanoidin fractions derived from two di ff erent types of cocoa beans by UHPLC-DAD-ESIHR-MS. Food Research International, 115(August 2018), 135-149. https://doi.org/10.1016/j.foodres.2018.08.028

Othman, A., Ismail, A., Abdul, N., \& Adenan, I. (2007). Food Chemistry Antioxidant capacity and phenolic content of cocoa beans. Food Chemistry, 100, 1523-1530. https://doi.org/10.1016/j.foodchem.2005.12.021

Owusu, M., Petersen, M. A., \& Heimdal, H. (2011). Effect of fermentation method, roasting and conching conditions on the aroma volatiles of dark chocolate. Journal of Food Processing and Preservation, 1-11. https://doi.org/10.1111/j.17454549.2011.00602. $x$

Páramo, D., García-Alamilla, P., Salgado-Cervantes, M. A., Robles-Olvera, V. J., Rodríguez-Jimenes, G. C., \& García-Alvarado, M. A. (2010). Mass transfer of water and volatile fatty acids in cocoa beans during drying. Journal of Food Engineering, 99(3), 276-283. https://doi.org/10.1016/j.jfoodeng.2010.02.028 
Parra, O., Gallego, A. M., Urrea, A., Rojas, L. F., Correa, C., \& Atehortúa, L. (2017). Biochemical precursor effects on the fatty acid production in cell suspension cultures of Theobroma cacao L. Plant Physiology and Biochemistry, 111, 59-66. https://doi.org/10.1016/j.plaphy.2016.11.013

Patras, M. A., Milev, B. P., Vrancken, G., \& Kuhnert, N. (2014). Identification of novel cocoa $\mathrm{fl}$ avonoids from raw fermented cocoa beans by HPLC - MS n. Food Research International Journal, 63, 353-359. https://doi.org/10.1016/j.foodres.2014.05.031

Pedan, V., Weber, C., Do, T., Fischer, N., Reich, E., Rohn, S., \& Andrew, S. (2017). HPTLC fi ngerprint pro fi le analysis of cocoa proanthocyanidins depending on origin and genotype. Food Chemistry, (August). https://doi.org/10.1016/j.foodchem.2017.08.109

Pereira-Caro, G., Borges, G., Nagai, C., Jackson, M. C., Yokota, T., Crozier, A., \& Ashihara, H. (2013). Profiles of Phenolic Compounds and Purine Alkaloids during the Development of Seeds of Theobroma cacao cv. Trinitario. Journal of Agricultural and Food Chemistry, 61, 427-434. https://doi.org/10.1021/jf304397m

Petersen, M. A., Blennow, A., \& Nielsen, D. S. (2014). Impact of starter cultures and fermentation techniques on the volatile aroma and sensory profile of chocolate. https://doi.org/10.1016/j.foodres.2014.04.032

Pinto-Mosquera, N. (2011). Propuesta de mejora a los factores que afectan la competitividad de la cadena productiva del cacao en grano de la empresa "Aroma Amazónico CIA. LTDA." Universidad de Las Américas.

Pontillon, J. (1997). Cacao et chocolat: Production, utilisation, caractéristiques. Paris: Tec et doc-Lavoisier.

Predan, G. M. I., Lazăr, D. A., \& Lungu, I. I. (2019). Cocoa industry-from plant cultivation to cocoa drinks production. Caffeinated and Cocoa Based Beverages: Volume 8. The Science of Beverages, 489-507. https://doi.org/10.1016/B978-012-815864-7.00015-5

PROECUADOR. (2013). Análisis del sector cacao y elaborados. Quito, Ecuador.

Quiñones, M., Miguel, M., \& Aleixandre, A. (2012). Los polifenoles, compuestos de origen natural con efectos saludables sobre el sistema cardiovascular. (1). https://doi.org/10.3305/nh.2012.27.1.5418

Ramli, N., Yatim, A. M., Said, M., \& Hok, H. C. (2001). HPLC Determination of 
Methylxanthines and Polyphenols Levels In Cocoa and Chocolate Products. Malaysian Journal of Analytical Sciences, 7(2), 377-386.

Riaño, N., Chica, M., Echeverri, L., Aguirre, J., Ortiz, A., Pineda, R., \& Olarte, N. (2016). Contenido de grasa total, perfil de Ácidos grasos y triglicéridos proveniente de cacaos finos de aroma: Colombia, Ecuador, Perú, Venezuela (Vol. 23).

Ribeiro, P. V. de M., Andrade, P. A., Hermsdorff, H. H. M., dos Santos, C. A., Cotta, R. M. M., Estanislau, J. de A. S. G., ... Rosa, C. de O. B. (2019). Dietary nonnutrients in the prevention of non-communicable diseases: Potentially related mechanisms. Nutrition, 66, 22-28. https://doi.org/10.1016/j.nut.2019.03.016

Rodríguez-Carrasco, Y., Gaspari, A., Graziani, G., Santini, A., \& Ritieni, A. (2018). Fast analysis of polyphenols and alkaloids in cocoa-based products by ultra- high performance liquid chromatography and Orbitrap high resolution mass spectrometry ( UHPLC-Q-Orbitrap-MS / MS ). Food Research International, 111(February), 229-236. https://doi.org/10.1016/j.foodres.2018.05.032

Romanens, E., Freimüller Leischtfeld, S., Volland, A., Stevens, M., Krähenmann, U., Isele, D., ... Miescher Schwenninger, S. (2019). Screening of lactic acid bacteria and yeast strains to select adapted anti-fungal co-cultures for cocoa bean fermentation. International Journal of Food Microbiology, 290(October 2018), 262272. https://doi.org/10.1016/j.ijfoodmicro.2018.10.001

Saltini, R., Akkerman, R., \& Frosch, S. (2013). Optimizing chocolate production through traceability: A review of the in fl uence of farming practices on cocoa bean quality. Food Control, 29(1), 167-187. https://doi.org/10.1016/j.foodcont.2012.05.054

Samaniego, I., Espín, S., Quiroz, J., Ortiz, B., Carrillo, W., García-Viguera, C., \& Mena, P. (2020). Effect of the growing area on the methylxanthines and flavan-3-ols content in cocoa beans from Ecuador. Journal OfFood Composition and Analysis, 1(January), 103448. https://doi.org/10.1016/j.jfca.2020.103448

Santana, P. (2017). Mucílago de cacao (Theobroma cacao I.), nacional y trinitario para la obtención de una bebida hidratante. Universidad Técnica Estatal de Quevedo.

Santos, D. S., Rezende, R. P., Santos, T. F. dos, Marques, E. de L. S., Ferreira, A. C. R., Silva, A. B. de C. e., ... Tavares Bisneto, J. D. (2020). Fermentation in fine cocoa type Scavina: Change in standard quality as the effect of use of starters yeast in fermentation. Food Chemistry, 328(October 2019), 7-12. https://doi.org/10.1016/j.foodchem.2020.127110 
Sarbu, I., \& Csutak, O. (2019). The microbiology of cocoa fermentation. In Caffeinated and Cocoa Based Beverages: Volume 8. The Science of Beverages. https://doi.org/10.1016/B978-0-12-815864-7.00013-1

Schinella, G., Mosca, S., Cienfuegos-jovellanos, E., Ángeles, M., Muguerza, B., Ramón, D., \& Luis, J. (2010). Antioxidant properties of polyphenol-rich cocoa products industrially processed. Food Research International, 43, 1614-1623. https://doi.org/10.1016/j.foodres.2010.04.032

Serra, D., Almeida, L. M., \& Dinis, T. C. P. (2019). Polyphenols as food bioactive compounds in the context of Autism Spectrum Disorders: A critical mini-review. Neuroscience and Biobehavioral Reviews, 102(March), 290-298. https://doi.org/10.1016/j.neubiorev.2019.05.010

Servent, A., Boulanger, R., Davrieux, F., Pinot, M. N., Tardan, E., Forestier-Chiron, N., \& Hue, C. (2018). Assessment of cocoa (Theobroma cacao L.) butter content and composition throughout fermentations. Food Research International, 107(2017), 675-682. https://doi.org/10.1016/j.foodres.2018.02.070

Silva, R. F. M., \& Pogǎcnik, L. (2020). Polyphenols from food and natural products: Neuroprotection and safety. (2010), 1-13.

Sirbu, D., Corno, M., Ullrich, M. S., \& Kuhnert, N. (2018). Characterization of triacylglycerols in unfermented cocoa beans by HPLC-ESI mass spectrometry. Food Chemistry, 254(February), 232-240. https://doi.org/10.1016/j.foodchem.2018.01.194

Steinberg, F. M., Bearden, M. M., \& Keen, C. L. (2003). Cocoa and chocolate flavonoids: implications for cardiovascular health. Journal of the American Dietetic Association, 103(2), 215.

Sudano, I., Flammer, A. J., Roas, S., Enseleit, F., Ruschitzka, F., Corti, R., \& Noll, G. (2012). Cocoa, Blood Pressure, and Vascular Function. Current Hypertension Reports, 14, 279-284. https://doi.org/10.1007/s11906-012-0281-8

Szajdek, A., \& Borowska, E. J. (2008). Bioactive compounds and health-promoting properties of Berry fruits: A review. Plant Foods for Human Nutrition, 63(4), 147153. https://doi.org/10.1007/s11130-008-0097-5

Teye, E., Huang, X., Sam-amoah, L. K., Takrama, J., Boison, D., Botchway, F., \& Kumi, F. (2015). Estimating cocoa bean parameters by FT-NIRS and chemometrics analysis. Food Chemistry, 176, 403-410. 
https://doi.org/10.1016/j.foodchem.2014.12.042

Tezara, W., De Almeida, J., Valencia, E., Cortes, J. L., \& Bolaños, M. J. (2015). Actividad fotoquímica de clones élite de cacao ( $\mathrm{d}$ L.) en el norte de la provincia de Esmeraldas. Investigación y Saberes, 4(3), 35-72.

Tezara, Wilmer, Urich, R., Jaimez, R., Coronel, I., Araque, O., Azócar, C., \& Chacón, I. (2016). Does griollo cocoa have the same ecophysiological characteristics as forastero? Botanical Sciences, 94(3), 563-574. https://doi.org/10.17129/botsci.552

Thuy, V. T., Zhao, J., \& Fleet, G. (2015). The effect of lactic acid bacteria on cocoa bean fermentation. International Journal of Food Microbiology, 205(May), 54-67. https://doi.org/10.1016/j.jifoodmicro.2015.03.031

Todorovic, V., Radojcic, I., Todorovic, Z., Jankovic, G., Dodevska, M., \& Sobajic, S. (2015). Journal of Food Composition and Analysis Polyphenols, methylxanthines, and antioxidant capacity of chocolates produced in Serbia. Journal of Food Composition and Analysis. https://doi.org/10.1016/j.jfca.2015.01.018

Torres-Moreno, M., Torrescasana, E., Salas-Salvadó, J., Blanch, C., Torres-Moreno, M., Torrescasana, E., ... Blanch, C. (2015). Nutritional composition and fatty acids profile in cocoa beans and chocolates with different geographical origin and processing conditions. Food Chemistry, 166, 125-132. https://doi.org/10.1016/j.foodchem.2014.05.141

Torres Murillo, M. (2016). Cadena de valor para la comercialización de cacao de los productores del recinto Tazone (UNIVERSIDAD CATÓLICA DEL ECUADOR SEDE ESMERALDAS DIRECCIÓN; https://doi.org/10.1017/CBO9781107415324.004

Vargas Jentzsch, P., Ciobotă, V., Salinas, W., Kampe, B., Aponte, M., Rösch, P., ... Ramos, L. A. (2016). Distinction of Ecuadorian varieties of fermented cocoa beans using Raman spectroscopy. Food Chemistry, 211, 274-280. https://doi.org/10.1016/j.foodchem.2016.05.017

Vera Chang J, Cabrera Verdezoto R, Veliz Zamora D, Segovia Freire G, Torres Navarrete Y, Pinargote Alava J, . (2018). Validación de tres métodos de propagación en cacao (Theobroma cacao L.) Nacional y Trinitario en Ecuador. Conamti, 5(01), 01-06.

Wang, G., Lee, A. S., Lewis, M., Kamath, B., \& Archer, R. K. (1999). Accelerated solvent extraction and gas chromatography/mass spectrometry for determination 
of polycyclic aromatic hydrocarbons in smoked food samples. Journal of Agricultural and Food Chemistry, 47(3), 1062-1066.

Wollgast, J., \& Anklam, E. (2000). Review on polyphenols in Theobroma cacao: changes in composition during the manufacture of chocolate and methodology for identification and quantification. Food Research International, 33.

Wood, G. A. R., \& Lass, R. A. (1985). Cocoa (4th Editio; Longman, Ed.). London, United Kingdom.

Yépez Rivadeneira, J. (2017). Caracterización del contenido de polifenoles: catequina, epicatequina y procianidinas B1, B2 y C1; en cacao CCN-51 de las principales zonas productoras del Ecuador. UNIVERSIDAD CENTRAL DEL ECUADOR.

Zambrano, G. (2018). Evaluación de la influencia del proceso de beneficio del cacao (Theobroma cacao) CCN-51 de altura en su calidad final, mediante el análisis físico, físico-químico y sensorial. UNIVERSIDAD CENTRAL DEL ECUADOR.

Zambrano Tenezaca, J. (2017). Caracterización físico química y nutricional de bebida de tomate de árbol con inclusión de cascarilla de cacao. Universidad Técnica de Machala.

Żyżelewicz, D., Wiesława, K., Oracz, J., Sosnowska, D., Grażyna, B., \& Nebesny, E. (2016). The in $\mathrm{fl}$ uence of the roasting process conditions on the polyphenol content in cocoa beans, nibs and chocolates. Food Research International Journal, 89, 918-929. https://doi.org/10.1016/j.foodres.2016.03.026 


\section{PUBLICACIÓN II}

How does the growing area condition the fat content and the fatty acid composition of Ecuadorian cocoa beans?

Iván Samaniego, Susana Espín, James Quiroz, Carmen Rosales, Wilman Carrillo, Pedro Mena, Cristina García-Viguera.

Journal of Food Sciences and Nutrition

Under review

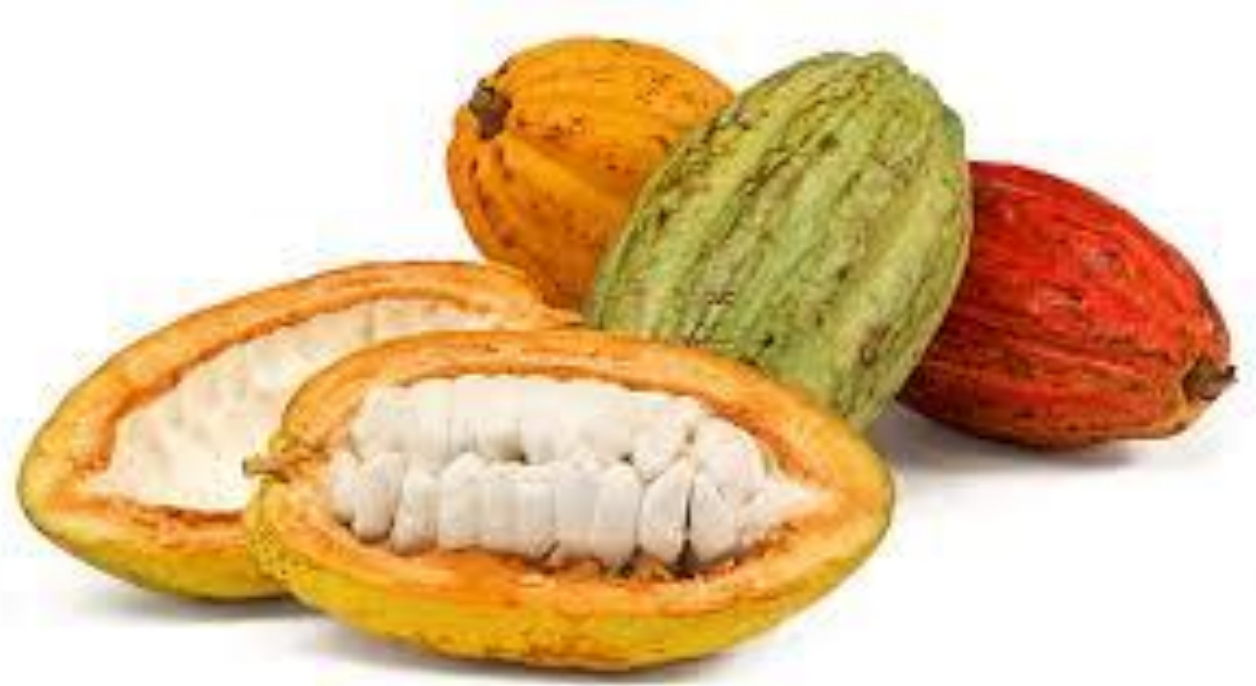




\section{Introduction}

Cocoa (Theobroma cacao L.) is the basic raw material in chocolate and other confectionary products containing chocolate. It is a key crop for developing countries in several world regions like Asia, Latin America, and Africa. Cocoa beans are commonly classified into 4 different varieties: Criollo, Forastero, Trinitario (an hybrid of Criollo and Forastero), and Ecuadorian Nacional (national in Spanish) (De Taeye et al. 2017). The majority of the world production corresponds to "bulk" grade cocoa beans, grown mainly in Africa and belonging to the variety Forastero. Criollo, Trinitario, and Nacional are however defined as high-quality ("fine" grade) varieties, Ecuador being the main producer worldwide of "fine" cocoa beans (Afoakwa et al. 2008; Dand 2011a; Exporters-Ecuador National Association of Cocoa 2016; Kongor et al. 2016; Vargas Jentzsch et al. 2016; International Cocoa Organization 2017). Contrary to other varieties, Nacional has a very short fermentation time and yields excellent beans in terms of flavour (Afoakwa et al. 2008; Motamayor et al. 2008; Afoakwa et al. 2011; Dand 2011b; Oracz \& Nebesny 2014; Kongor et al. 2016). Pure Nacional cocoa genotypes are currently scarce and most of the orchards use natural hybrids introduced from Trinidad and Venezuela, which forms the Nacional and Trinitario complex (also known as "Arriba" cocoa). Fine "Arriba" cocoa beans present distinguishing organoleptic properties and are highly demanded by the chocolate manufacturing (Afoakwa et al. 2008; Caligiani et al. 2014; Tezara et al. 2015).

Cocoa butter is the natural fat extracted from cocoa beans. This lipid fraction constitutes around $50 \%$ of the total weight of dry grains, with some variations that include values between 48 and $58 \%$, depending on the variety of cocoa and the production area (Zyzelewicz et al. 2014). Cocoa butter is the most valuable component of cocoa beans and it is expensive compared to other vegetable fats and oils due to its physical (rheology and texture) and chemical properties, as well as its organoleptic qualities. These qualities make cocoa fat very suitable for the manufacture of a wide variety of products in the chocolate/confectionary, cosmetic and pharmaceutical industries (Jahurul et al. 2013; CAOBISCO/ECA/FCC 2015; Torres-Moreno et al. 2015; Riaño et al. 2016). The composition in fatty acids (FA) of cocoa butter is, thus, of great interest for the industry as their occurrence and positional distribution in the triacylglycerol molecules determine the physical and thermal properties of cocoa butter as well as the chocolate processing (Foubert et al. 2004; Jahurul et al. 2013; Zyzelewicz et al. 2014; Torres-Moreno et al. 2015) The FA profile of cocoa butter is constituted mainly by palmitic acid (C16:0), stearic acid (18:0), and oleic acid (C18:1), 
with linoleic acid (C18:2) and arachidic acid (C20:0) as minor but relevant FA (Naik \& Kumar 2014; Torres-Moreno et al. 2015; Riaño et al. 2016; Parra et al. 2017; Servent et al. 2018). This FA composition also conditions the nutritional value of cocoa beans and their related chocolate products according to its content in saturated FA (SFA, C16:0, C18:0, and C20:0), monounsaturated FA (MUFA, C18:1) and polyunsaturated FA (PUFA, C18:2) (Torres-Moreno et al. 2015; Caprioli et al. 2016). For instance, it has been reported that Ecuadorian chocolates present a healthier FA profile than chocolates produced from cocoas grown in Ghana (Torres-Moreno et al. 2015). In this regard, it should be highlighted that the biochemical composition of cocoa fat depends on several factors, such as: variety, age of the plantation, maturation of the fruits, environmental conditions and growing area (Lehrian et al. 1980; Hernandez et al. 1991; Foubert et al. 2004; Jahurul et al. 2013; CAOBISCO/ECA/FCC. 2015; Caprioli et al. 2016). Knowledge in the FA composition of cocoa butter is thus of great interest for processing and quality control, research laboratories, and to better define the nutritional properties of cocoa-derived products (Torres-Moreno et al. 2015; Caprioli et al. 2016).

Despite the consolidated cultivation of Nacional cocoa beans in Ecuador and its economic importance at world level, little is known about the differences on the fat content and the FA profile of cocoas grown in different Ecuadorian areas. A preliminary study has reported a high variability among growing areas (Jimenez, J., Amores, F., \& Solórzano et al. 2014), but a complete evaluation of the impact of the wide edaphoclimatic conditions between Ecuadorian regions on the fat content and the FA profile of Nacional cocoa is still missing. This study aimed at filling that gap, assessing the fat content and the FA composition of the main producing areas of Ecuador (the Pacific coast and the Amazon region). Smaller geographical units than regions, as provinces and cantons, were also considered to better understand the ecological characteristics that may condition the development of specific FA profiles.

\section{Material and methods}

\subsection{Material and sample collection}

Cocoa samples (Nacional variety from the National x Trinitario complex, "Arriba" flavour) were collected from 85 selected farms. Farms were representative of the main Ecuadorian growing areas: Pacific Coast and Amazonian regions. The cocoa-growing area of Ecuador corresponding to the Pacific Coast is located from the west part of the Andes to the Pacific Ocean, while the Amazonian region is located from the east part of the Andes to the Peruvian and Colombian borders. 


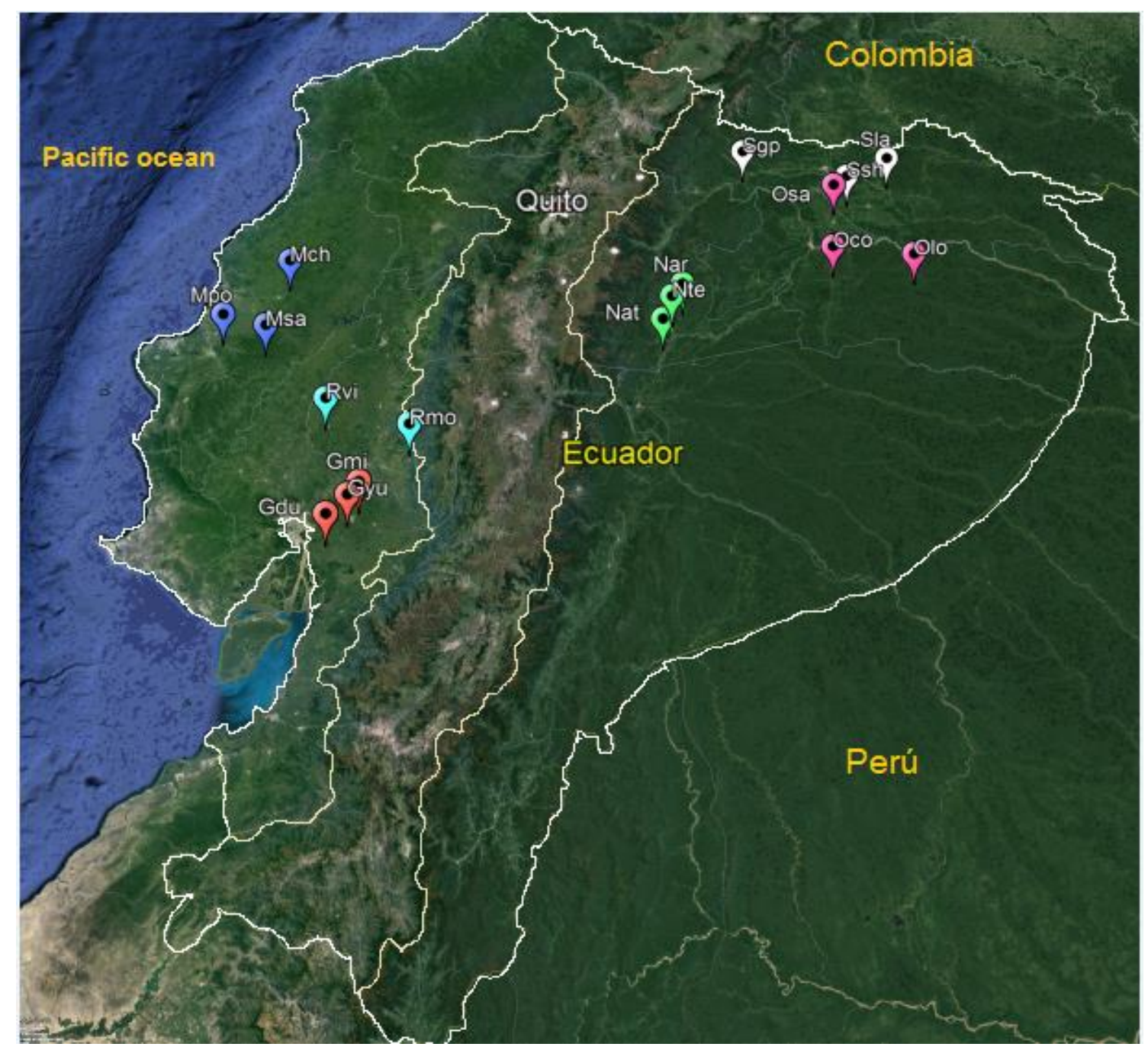

Figure 1. Geographical location of Ecuadorian cocoa-growing areas under study

The three highest producing provinces were selected within each region on the basis of the Ecuadorian cocoa map: Guayas, Los Ríos, and Manabí (accounting for $70 \%$ of the national production) in the Pacific Coast region; Sucumbíos, Francisco de Orellana, and Napo ( $6 \%$ of production at national level) in the Amazonian region (Ministry of Agriculture and Livestock 2016). Three cantons, with the highest productions, were selected within each province (except for Los Ríos province, where only 2 cantons were taken into account), for a total of up to 17 cantons (Table 1, Supplementary Figure S1), and 5 representative farms were chosen for each canton. Two samples of 20 cocoa fruits belonging to 20 cacao trees (1 fruit/tree) randomly chosen were harvested within each farm to get approximately $1 \mathrm{~kg}$ of fermented, dried beans per sample. The harvested fruits were placed in jute bags and transported to the PostHarvest Cocoa Treatment Center of the South Littoral Experimental Station of the 
INIAP (Ecuadorian National Institute of Agricultural Research, Yaguachi canton, province of Guayas) before processing.

Table 1. Growing areas for the Ecuadorian cocoa beans collected. Cantons are abbreviated in brackets.

\begin{tabular}{|c|c|c|c|c|c|}
\hline N. & Region & Province & Canton & $\begin{array}{l}\text { Altitude } \\
\text { (m.a.s.l) }\end{array}$ & $\begin{array}{c}\text { Temperature, } \\
\text { annual } \\
\text { average } \\
\left({ }^{\circ} \mathrm{C}\right) \\
\end{array}$ \\
\hline 1 & & & Milagro (PGmi) & $25-27$ & $23-29$ \\
\hline 2 & & Guayas & Yaguachi (PGya) & $13-31$ & $23-27$ \\
\hline 3 & & & Durán (PGdu) & $8-15$ & $25-32$ \\
\hline 4 & Pacific & & Montalvo (PRmo) & $133-148$ & $22-26$ \\
\hline 5 & Coast & Los Ríos & Vinces (PRvi) & $11-25$ & $24-34$ \\
\hline 6 & & & Santa Ana (PMsa) & $61-182$ & $24-31$ \\
\hline 7 & & Manabí & Portoviejo (PMpo) & $25-42$ & $24-31$ \\
\hline 8 & & & Chone (PMch) & $24-30$ & $23-31$ \\
\hline 9 & & & El Coca (AOco) & $282-294$ & $18-28$ \\
\hline 10 & & Francisco de Orellana & Loreto (AOlo) & $275-290$ & $22-31$ \\
\hline 11 & & & $\begin{array}{l}\text { Joya de los Sachas } \\
\text { (AOsa) }\end{array}$ & $368-422$ & $19-29$ \\
\hline 12 & & & Lago Agrio (ASla) & $250-268$ & $21-30$ \\
\hline 13 & Amazonian & Sucumbíos & Shushufindi (ASsh) & $283-285$ & $25-32$ \\
\hline 14 & & & Gonzalo Pizarro (ASgp) & $385-615$ & $24-32$ \\
\hline 15 & & & Tena (ANte) & $300-500$ & $21-29$ \\
\hline 16 & & Napo & Arosemena Tola (ANat) & $542-735$ & $21-29$ \\
\hline 17 & & & Archidona (ANar) & $665-710$ & $21-29$ \\
\hline
\end{tabular}

\subsection{Post-harvest processing and sample preparation}

Fermentation and drying of the cocoa samples was performed as previously described (Samaniego et al. 2020). Briefly, beans were extracted with the mucilage and a micro fermentation technique was carried out in laurel wood boxes of $120 \times 120 \times 80 \mathrm{~cm}$ (height $x$ width $x$ depth), with a capacity of $120 \mathrm{~kg}$. Each box contained 12 samples and the fermentation process last $96 \mathrm{~h}$, with a mass removal at $48 \mathrm{~h}$, as required for Nacional cacao. After that, a sun-drying step was applied in cement slots until reaching a proper bean humidity ( $7 \%$ approximately). Samples were then placed in paper bags and transported to the LSAIA Food Analysis and Research Service Laboratory of the Santa Catalina Experimental Station of INIAP. 
Up to $250 \mathrm{~g}$ of each sample were used for analysis. Husks were manually removed using a scalpel and nibs were frozen under liquid nitrogen. Frozen samples were milled and sieved (Ultra Centrifugal mill Retsch ZM 200, Hann, Germany) to obtain a fine powder (particle size smaller than $1 \mathrm{~mm}$ ). The ground cocoa was then stored in hermetic bottles at refrigeration temperature $\left(5^{\circ} \mathrm{C}\right)$ until analyses.

\subsection{Reagents}

All chemicals and solvents used in this study were of analytical grade and were purchased from Merck (Darmstadt, Germany). Deionized water was prepared with a Milli-Q water purification system (Millipore, Sao Paulo, Brazil). The fatty acid methyl ester (FAME) standard FAME Mix C4 to C24 was obtained from Supelco (Pennsylvania, USA).

\subsection{Determination of total fat (TF) content}

TF was evaluated by continuous extraction with organic solvent by the Soxhlet method, using an official protocol described in the manual of chemical parameters associated with Ecuadorian cocoa quality (Espín \& Samaniego 2016) and based on method \#37 of the International Office of Cocoa, Chocolate and Sugar Confectionery (IOCCC) (1996). Briefly, $5 \mathrm{~g}$ of cocoa were weighed on a cellulose thimble $(33 \times 88 \mathrm{~mm})$, covered with cotton, and subjected to a continuous extraction process with $200 \mathrm{~mL}$ of petroleum ether at $90^{\circ} \mathrm{C}$ in a Selecta Soxhlet extraction system (Barcelona, Spain) for $16 \mathrm{~h}$. Subsequently, thimbles were removed with the defatted sample from the extraction system and the solvent was recovered. The distillation balloons with the lipid fraction were placed in an oven (Precision Scientific, Chicago, USA) at $105{ }^{\circ} \mathrm{C}$ for $2 \mathrm{~h}$ to remove the solvent residue, cooled in a desiccator for $2 \mathrm{~h}$, and weighed on a Shimadzu model AUX 220 analytical balance (Kyoto, Japan). The TF content was calculated by difference in weight between the distillation balloon with fat and the empty distillation balloon in relation to the sample weight used. Analyses were performed in duplicate for each replicate of farm samples. The result was reported in grams of fat per $100 \mathrm{~g}$ of dry sample $(g / 100 g$ DW).

\subsection{Fatty acid (FA) composition}

The analysis of the content of FAMEs in cocoa fat was carried out using the official method 996.06 (AOAC International, 2005), with minor modifications (Espín \& Samaniego 2016). The fatty acid esters (FAMEs) were prepared weighing $0.05 \mathrm{~g}$ of 
cocoa fat previously extracted in a $10 \mathrm{~mL}$ test tube with screw cap. $1 \mathrm{~mL}$ of $0.5 \mathrm{M}$ potassium hydroxide solution in methanol was added and heated in a Memmert water bath (Schwabach, Germany) at $100{ }^{\circ} \mathrm{C}$ for $10 \mathrm{~min}$. It was subsequently cooled at room temperature for 5 minutes. Then, $0.4 \mathrm{~mL}$ of hydrochloric acid solution in methanol $(4: 1$ $\mathrm{v} / \mathrm{v}$ ) was added and the sample was reheated again in the water bath at $100{ }^{\circ} \mathrm{C}$ for 25 min. The sample was cooled at room temperature for $10 \mathrm{~min}, 3 \mathrm{~mL}$ of hexane were added and stirred for 30 secs in a Thermolyne vortex tube agitator (lowa, USA), and 3 $\mathrm{mL}$ of water were added and left at rest until the organic phase of the aqueous phase separates. The organic phase containing the FAMEs was recovered. This process was repeated three times. The organic phase was then concentrated to dryness with a stream of nitrogen at room temperature and finally reconstituted with $2 \mathrm{~mL}$ of hexane for chromatographic analysis.

The chromatographic analysis was performed using an Agilent Technologies 7890A gas chromatographer (GC) coupled to a flame ionization detector (FID), controlled by ChemStation Productivity software (Agilent Technologies, Waldbronn, Germany). The separation was performed on a SP TM 2560 fused silica capillary column (100 m x 0.25 mm I.D., $0.2 \mu \mathrm{m}$ film thickness; Supelco, Pennsylvania, USA). Two $\mu \mathrm{L}$ of sample was injected into a split/splitless injector at a temperature of $260{ }^{\circ} \mathrm{C}$. The $\mathrm{GC}$ was configured with helium as a carrier gas at a constant flow of $1.07 \mathrm{~mL} / \mathrm{min}$. The column oven was programmed as follows: $140{ }^{\circ} \mathrm{C}(5 \mathrm{~min})$; to $240{ }^{\circ} \mathrm{C}(5 \mathrm{~min})$ at $4{ }^{\circ} \mathrm{C} / \mathrm{min}$. Identification was carried out by comparing the retention times of the peaks with their respective standards and their column elution order, using a FAME mix standard from C4 to C24 as reference. Quantification was performed by the external standard method, comparing the area of the sample with the area of its respective standard. Results were expressed as gram of each FA per $100 \mathrm{~g}$ of cocoa fat (CF). Analyses were performed in duplicate for each replicate of farm samples.

\section{Statistical analysis}

Data is reported as mean $\pm S D$ ( $n=5$ farms per canton). Statistical differences between regions were assessed by $t$-tests, while for provinces and cantons were evaluated by means of one-way ANOVA using Statistica 10.0 software for Windows (StatSoft, Paris, France). Differences among the means were evaluated using the Tukey's test (multiple range test) at a 95\% confidence level. Pearson's correlation and principal component analysis (PCA) with varimax and the subsequent PC comparisons by $t$-test (between regions) or ANOVA with Tukey's post-hoc test (among provinces and cantons) were 
carried out using the IBM SPSS Statistics 25 software package (SPSS Inc., Chicago, IL, USA).

\section{Results and discussion}

\subsection{Total fat content in Nacional cocoa beans}

Fat is the most abundant nutrient within cocoa chemical composition (Afoakwa et al. 2011; CAOBISCO/ECA/FCC 2015; Torres-Moreno et al. 2015; Riaño et al. 2016). The average TF content evaluated in Nacional cocoa beans from 17 cantons of the Pacific Coast and the Amazon region of Ecuador varied between $45.61 \pm 1.27$ to $52.13 \pm 0.58$ $\mathrm{g} / 100 \mathrm{~g}$ DW (Table 2). TF content was higher in the provinces and cantons from the Amazonian region than in those from the Pacific Coast $(p<0.001)$, differences among provinces and cantons also being statistically significant $(p<0.05)$. Values ranged between $50.42 \pm 1.17$ and $52.13 \pm 0.58 \mathrm{~g} / 100 \mathrm{~g} \mathrm{DW}$ for Amazonian cantons, while from $45.61 \pm 1.27$ to $48.60 \pm 1.92 \mathrm{~g} / 100 \mathrm{~g}$ DW in the Pacific Coast cantons (Table 2). The average content of TF per province varied in the following order: Francisco de Orellana $=$ Napo $=$ Sucumbios $>$ Manabí $=$ Los Ríos > Guayas. Regarding cantons, no differences were found within cantons belonging to the Amazonian region, while cantons from the Pacific Coast showed a higher variability (Table 2). Nevertheless, cantons within the same province presented similar values among them for the majority of the provinces. These results confirmed that there is an effect of the growing area on the content of TF for Ecuadorian Nacional cocoa beans. This fact, besides conditioning the quantity of cocoa butter that may be extracted, may directly influence the quality of fat in cocoa beans and manufacturing processes (D W Lehrian et al. 1980; Pontillon 1997; Brunetto et al. 2007; Belščak et al. 2009; Carrillo et al. 2014; CAOBISCO/ECA/FCC 2015; Torres-Moreno et al. 2015; Riaño et al. 2016).

Results for Nacional cocoa beans were close to the ranges reported by several authors for other Ecuadorian samples, with the content of TF in fermented and dried cocoa from varying between 43.4 to $51.9 \mathrm{~g} / 100 \mathrm{~g}$ DW (CAOBISCO/ECA/FCC 2015; TorresMoreno et al. 2015; Riaño et al. 2016; Servent et al. 2018). A study by Jimenez et al. (2014), conducted with samples of Ecuadorian Nacional cocoa collected in 6 different production areas, showed that the TF ranged from 44.3 to $47.3 \mathrm{~g} / 100 \mathrm{~g} \mathrm{DW}$. 
Table 2. Content of total fat in Nacional cocoa from the main growing areas of Ecuador

\begin{tabular}{cclll}
\hline N. & Province & Canton & \multicolumn{2}{l}{ Total fat (g/100 g) } \\
\hline 1 & Guayas & Milagro & $46.29 \pm 0.92$ & $\mathrm{C} \mathrm{d}$ \\
2 & (Pac. Coast) & Yaguachi & $46.31 \pm 1.29$ & $\mathrm{C} \mathrm{cd}$ \\
3 & & Durán & $45.61 \pm 1.27$ & $\mathrm{C} \mathrm{d}$ \\
\hline 4 & Los Ríos & Montalvo & $47.42 \pm 2.26$ & $\mathrm{~B} \mathrm{~cd}$ \\
5 & (Pac. Coast) & Vinces & $47.28 \pm 1.71$ & $\mathrm{~B} \mathrm{~cd}$ \\
\hline 6 & Manabí & Santa Ana & $48.60 \pm 1.92$ & $\mathrm{~B} \mathrm{bc}$ \\
7 & (Pac. Coast) & Portoviejo & $47.83 \pm 1.22$ & $\mathrm{~B} \mathrm{~cd}$ \\
8 & & Chone & $46.46 \pm 2.75$ & $\mathrm{~B} \mathrm{~cd}$ \\
\hline 9 & Francisco de & El Coca & $51.63 \pm 2.02$ & $\mathrm{~A} \mathrm{a}$ \\
10 & Orellana & Loreto & $51.23 \pm 1.19$ & $\mathrm{~A} \mathrm{a}$ \\
11 & (Amazon) & Sachas & $52.13 \pm 0.58$ & $\mathrm{~A} \mathrm{a}$ \\
\hline 12 & Sucumbíos & Lago Agrio & $50.44 \pm 0.68$ & $\mathrm{~A} \mathrm{ab}$ \\
13 & (Amazon) & Shushufindi & $50.83 \pm 0.84$ & $\mathrm{~A} \mathrm{ab}$ \\
14 & & Gonzalo Pizarro & $50.42 \pm 1.17$ & $\mathrm{~A} \mathrm{ab}$ \\
\hline 15 & Napo & Tena & $51.81 \pm 1.67$ & $\mathrm{~A} \mathrm{a}$ \\
16 & (Amazon) & Arosemena Tola & $51.25 \pm 1.11$ & $\mathrm{~A} \mathrm{a}$ \\
17 & & Archidona & $51.28 \pm 0.77$ & $\mathrm{~A} \mathrm{a}$ \\
\hline
\end{tabular}

Results are expressed as mean $\pm \mathrm{SD}(n=5)$. Different uppercase letters indicate statistically significant differences $(p<0.05)$ among provinces; different lowercase letters indicate significant differences $(p<0.05)$ among cantons.

These limits were slightly lower than those obtained in this study and may be related to the differences in the sampling areas. On the contrary, Steinberg, Bearden, \& Keen (2003) indicated that the fat content in fresh unroasted cocoa varies from 50 to 57 $\mathrm{g} / 100 \mathrm{~g}$ DW, values above those collected in this work. It should be however mentioned that the variety is key in defining the fat content of cocoa samples and that the highest fat contents are usually reported for Forastero type cocoas (D W Lehrian et al. 1980; Pontillon 1997; CAOBISCO/ECA/FCC 2015). Forastero cocoa beans from West Africa had fat contents of between $56 \%$ and $58 \%$ (Wood \& Lass (1985)). These higher fat contents also condition the quality and processing of the cocoa beans, as the greater the amount of TF in the grain, the longer the fermentation process will be (CAOBISCO/ECA/FCC 2015). In Criollo type cocoas, the fat content tends to be lower than Forastero, estimated to be approximately 53\% (Wood \& Lass 1985). Fat contents lower than $50 \%$ have also been reported in studies carried out in collections of ancient Criollos cocoas in Venezuela (Wood \& Lass 1985). Overall, a comprehensive analysis 
of fine cocoa beans grown in different Latin American countries revealed that the fat content ranged from 50.7 (Venezuela) to 53.6 (Peru) g/100g DW, with Ecuadorian fine cocoas having intermediate values (Riaño et al. 2016). These results were also in line with the study carried out for fine cocoas from Ecuador (Trinitario), Dominican Republic (Trinitario), and Madagascar (Criollo) by Servent et al. (2018), who reported that values ranged between $47.1 \%$ for the Ecuadorian samples and $53.0 \%$ for Dominican ones, with samples from Madagascar having a 52.2\% fat content. Last, besides genotype, variation in the fat content of cocoa beans could be related to other factors such as the plant age, growing practices, post-harvest processing, and environmental conditions (Servent et al. 2018).

\subsection{Fatty acid composition}

Due to the importance of the FA composition on the technological properties and nutritional quality of cocoa fat, the FA profile and content of Nacional cocoas collected in 17 Ecuadorian cantons were determined (Tables 3 and 4). The FA detected were C14:0 (myristic acid), C15:0 (pentadecanoic acid), C16:0 (palmitic acid), C16:1 (palmitoleic acid), C17:0 (margaric acid), C18:0 (stearic acid), C18:1 (oleic acid), C18:2 (linoleic acid), C18:3 (linolenic acid), C20:0 (arachidic acid), C20:1 (eicocenoic acid); C22:0 (behenic acid), C23:0 (tricosanoic acid), and C24:0 (lignoceric acid), fully in line with previous reports assessing the FA profile of cocoa fat (Żyżelewicz et al. 2018). The most important FA according to their average content $(n=85)$ in Ecuadorian Nacional cocoa beans were the SFA stearic and palmitic acids (34.13 \pm 1.05 and 28.63 $\pm 1.01 \mathrm{~g} / 100 \mathrm{~g} \mathrm{CF}$, respectively), the MUFA oleic acid (32.29 $\pm 0.95 \mathrm{~g} / 100 \mathrm{~g} \mathrm{CF})$, and the PUFA linoleic acid $(2.60 \pm 0.47 \mathrm{~g} / 100 \mathrm{~g} \mathrm{CF})$, which corresponded to $97.65 \%$ of the total FA. In the remaining fraction, arachidic acid presented the average highest content, with $1.01 \pm 0.17 \mathrm{~g} / 100 \mathrm{~g} \mathrm{CF}$, the other FA having contents in the range of 0.018 \pm 0.003 (tricosanoic acid) to $0.298 \pm 0.064 \mathrm{~g} / 100 \mathrm{~g} \mathrm{CF}$ (palmitoleic acid). These results were consistent with previous studies accounting for the most representative FA of cocoa fat (Foubert et al. 2004; Jahurul et al. 2013; Naik \& Kumar 2014; Torres-Moreno et al. 2015; Parra et al. 2017; Servent et al. 2018), although some differences with the ranges reported in the literature were found. For instance, Jahurul et al. (2013), reported higher ranges in Ecuadorian cocoas for stearic (34.6-36\%), oleic (34.6$34.9 \%$ ), and linoleic acid (2.6-3.0\%), while a lower range for palmitic acid (25.2-25.6\%). Servent et al. (2018), also provided slightly higher amounts of stearic acid (35.7\%), while average values for palmitic, oleic and linoleic acids were quite similar $(28.3,33.5$, and $2.5 \%$, respectively) to our data. On the contrary, the Ecuadorian cocoa analysed 
by Torres-Moreno, Torrescasana, Salas-Salvadó, Blanch, et al. (2015), presented lower average contents of palmitic (27.6\%) and stearic (33.8\%) acids but higher amounts of oleic acid (34.7\%). The information provided by Riaño et al. (2016), in a study conducted on 18 samples of Ecuadorian cocoa, was within the ranges obtained in this study. Therefore, although some variation among studies has been reported, data for the FA profile of Ecuadorian cocoas are pretty homogeneous when compared to the values reported for other varieties or producing area. This FA composition gives cocoa fat a specific degree of crystallization and its melting characteristics in the mouth (Naik \& Kumar 2014), confirming that Ecuadorian Nacional cocoa fat represents a good quality raw material for the elaboration of chocolates.

The effect of the growing area on the content of FA in fermented and dried Nacional cocoa beans revealed differences for some FA at the three geographical levels (region, province, and canton). Statistically significant differences were found for C14:0, C16:1, C17:0, C18:2, C20:0, and C22:0 at region level ( $p<0.001$ for all these FA): C14:0, C16:1, C20:0, and C22:0 amounts were higher in the Pacific Coast than in the Amazonian region, while $\mathrm{C} 17: 0$ and $\mathrm{C} 18: 2$ contents were higher in the Amazonian region. Differences among provinces and cantons $(p<0.05)$ were found for the following FA: C14:0, C16:0, C16:1, C17:0, C18:0, C18:1, C18:2, C20:0, C22:0, and C23:0 (Tables 3 and 4). Focusing on the most representative SFA (Table 3), it was established that, for palmitic acid (C16:0), Guayas and Francisco de Orellana were the provinces with the highest values, while the lowest was found for the Manabi province. In contrast, Manabí was the province with the highest values of stearic acid. The Amazonian province Napo presented the lowest values for arachidic acid while Pacific provinces Guayas and Manabí registered the highest values. Regarding cantons and main SFA, values for palmitic acid were quite similar for most of the cantons, while the content of stearic acid presented a higher variation among cantons, even for cantons within the same province (Table 3). In the case of the main unsaturated FA (Table 4), the cocoas collected in Los Ríos province had the highest oleic acid amounts, whereas the lowest values were found for the Manabí and Sucumbíos provinces. Linoleic acid contents were higher in the Amazonian province Napo while the lowest amounts were found in the province Guayas (Pacific Coast). The content of unsaturated FA changed among cantons, but most of the cantons belonging to the same province presented similar values for both oleic and linoleic acids (Table 4). 
Table 3. Saturated Fatty Acid content (g FA/100 g CF) in Nacional cocoa from the main production areas of Ecuador

\begin{tabular}{|c|c|c|c|c|c|c|c|c|c|c|c|c|c|c|c|c|c|c|}
\hline \multirow{2}{*}{$\frac{\text { N. }}{1}$} & \multirow{2}{*}{$\frac{\text { Province }}{\text { Guayas }}$} & \multirow{2}{*}{$\frac{\text { Canton }}{\text { Milagro }}$} & \multicolumn{2}{|c|}{ C14:0* } & \multirow{2}{*}{$\frac{\text { C15:0** }}{0.019 \pm 0.004}$} & \multicolumn{2}{|c|}{ C16:0* } & \multicolumn{2}{|c|}{ C17:0* } & \multicolumn{2}{|l|}{ C18:0* } & \multicolumn{2}{|c|}{ C20:0* } & \multicolumn{2}{|c|}{ C22:0* } & \multirow{2}{*}{$\frac{\text { C23:0* }}{0.018 \pm 0.005}$} & \multirow[b]{2}{*}{$a$} & \multirow{2}{*}{$\frac{\text { C24:0** }}{0.124 \pm 0.033}$} \\
\hline & & & $0.057 \pm 0.010$ & $\mathrm{~B}$ bcd & & .764 & $\mathrm{~A} \mathrm{a}$ & (2008 & $\mathrm{cd}$ & 37 & $\mathrm{~B} c d$ & 95 & $A a b c$ & 48 & $\mathrm{~A} a \mathrm{~b}$ & & & \\
\hline 2 & st) & Yaguachi & $58 \pm 0.009$ & B bcd & $0.020 \pm 0.005$ & $9.031 \pm 0.635$ & $\mathrm{~A} a b$ & $0.216 \pm 0.043$ & bcd & $33.751 \pm 0.964$ & B bcd & $1.100 \pm 0.209$ & $A a b$ & $0.199 \pm 0.041$ & $\mathrm{~A} \mathrm{a}$ & $0.018 \pm 0.005$ & $A B a$ & $0.121 \pm 0.025$ \\
\hline 3 & & Durán & $0.060 \pm 0.013$ & B bcd & $0.021 \pm 0.003$ & $28.903 \pm 0.775$ & $\mathrm{~A} a b$ & $0.215 \pm 0.046$ & B bcd & $34.090 \pm 0.814$ & $\mathrm{~B} \mathrm{bcd}$ & $1.111 \pm 0.267$ & A ab & $0.199 \pm 0.046$ & $\mathrm{~A} a b$ & $0.019 \pm 0.004$ & $\mathrm{Ba}$ & $0.119 \pm 0.031$ \\
\hline 4 & Los Ríos & onta & $667 \pm 0.015$ & B abcd & $0.019 \pm 0.003$ & $28.785 \pm 0.489$ & $\mathrm{~B} \mathrm{ab}$ & $0.213 \pm 0.031$ & $\mathrm{~B} \mathrm{bcd}$ & $33.152 \pm 0.606$ & $B d$ & $0.990 \pm 0.123$ & $A B$ abcd & $187 \pm 0.032$ & $A B a b$ & $0.017 \pm 0.003$ & $A B a b$ & 0.125 \\
\hline 5 & (Pac. Coast) & Vinces & $1+0016$ & $\mathrm{~B} \mathrm{bcd}$ & $0.019 \pm 0.005$ & $.825 \pm 0.957$ & Bah & $205 \pm 0.028$ & cy & $1 \pm 1.200$ & $\mathrm{~B} \mathrm{bcd}$ & $.064 \pm 0.110$ & $\mathrm{AB} a b c$ & 21 & $A B a b$ & .002 & $\mathrm{Ba}$ & 18 \\
\hline 6 & anabí & Santa Ana & $0.088 \pm 0.025$ & $\mathrm{Aa}$ & $0.021 \pm 0.002$ & $26.489 \pm 1.473$ & $\mathrm{Cc}_{\mathrm{c}}$ & $0.193 \pm 0.015$ & $B d$ & $35.965 \pm 0.991$ & $A a b$ & $1.043 \pm 0.041$ & A abcd & $0.179 \pm 0.008$ & $A B a b$ & $0.020 \pm 0.002$ & $\mathrm{~A} \mathrm{a}$ & $0.108 \pm 0.007$ \\
\hline 7 & $(F$ & Portoviejo & $0.075 \pm 0,017$ & $A a b c$ & $0.025 \pm 0.004$ & $28.419 \pm 1.438$ & $\mathrm{Cab}$ & $0.218 \pm 0.026$ & B bcd & $34.700 \pm 1.219$ & $A a b c$ & $1.148 \pm 0.076$ & $\mathrm{~A} \mathrm{a}$ & $0.188 \pm 0.009$ & $A B a b$ & $0.021 \pm 0.002$ & $\mathrm{~A} \mathrm{a}$ & $0.103 \pm 0.014$ \\
\hline 8 & & Chone & $78 \pm 0$ & $\mathrm{~A} a b$ & 0. & 7 & $\mathrm{C}_{\mathrm{C}}$ & 4 & $B=$ & 5 & $A=$ & 31 & $\mathrm{~A}$ & 20 & $\mathrm{~B}$ ab & 02 & 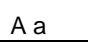 & 0125 \\
\hline 9 & Francisco & El Coca & $0.056 \pm 0.023$ & $\mathrm{~B} \mathrm{~cd}$ & $0.019 \pm 0.004$ & $29.171 \pm 0.426$ & $\mathrm{~A} a b$ & $0.240 \pm 0.033$ & A abcd & $33.055 \pm 0.797$ & $B d$ & $0.901 \pm 0.076$ & B C bcd & $0.157 \pm 0.015$ & $\mathrm{Bb}$ & $0.013 \pm 0.003$ & $\mathrm{Bb}$ & $0.107 \pm 0.018$ \\
\hline 10 & de & L & $0.062 \pm 0.010$ & $\mathrm{~B}$ bcd & $0.021 \pm 0.003$ & $29.158 \pm 0.532$ & $\mathrm{~A} a b$ & $0.278 \pm 0.037$ & $\mathrm{~A} \mathrm{a}$ & $34.327 \pm 0.473$ & $\mathrm{~B}$ bcd & $0.945 \pm 0.091$ & $\mathrm{BC}$ abcd & $0.175 \pm 0.017$ & $\mathrm{~B} a \mathrm{~b}$ & $0.019 \pm 0.002$ & $\mathrm{~B} \mathrm{a}$ & $0.123 \pm 0.011$ \\
\hline 11 & (Amazon) & ( & $54 \pm 0.011$ & 5 & $0.029 \pm 0.003$ & $28.850 \pm 0.733$ & $\mathrm{~A} a b$ & $0.259 \pm 0.037$ & A douc & $33.705 \pm 0.668$ & Dou & $0.976 \pm 0.121$ & $\mathrm{BC}$ abcd & $0.175 \pm 0.024$ & Dov & $0.017 \pm 0.002$ & on & .025 \\
\hline 12 & Sucumbíos & Lago Agrio & $0.059 \pm 0.006$ & B bod & $0.019 \pm 0.001$ & $28.470 \pm 0.550$ & $A B a b$ & $0.247 \pm 0.049$ & A abcd & $34.458 \pm 0.676$ & $\mathrm{~B} b \mathrm{c}$ & $0.964 \pm 0.069$ & $\mathrm{BC}$ abcd & $0.173 \pm 0.014$ & $A B a b$ & $0.018 \pm 0.003$ & $\mathrm{~B} \mathrm{ab}$ & $0.114 \pm 0.014$ \\
\hline 13 & (Amazon) & Shush & $0.056 \pm 0.009$ & $\mathrm{~B} c d$ & $0.020 \pm 0.001$ & $29.042 \pm 0.664$ & $A B a b$ & $0.279 \pm 0.019$ & $\mathrm{~A} \mathrm{a}$ & $33.552 \pm 0.798$ & $\mathrm{~B} c d$ & $1.067 \pm 0.094$ & $\mathrm{BC} a b c$ & $0.194 \pm 0.016$ & $A B a b$ & $0.017 \pm 0.002$ & $\mathrm{~B} \mathrm{a}$ & $0.142 \pm 0.035$ \\
\hline 14 & & Gonzalo Pizarro & $00 \pm 0.012$ & u & $0.020 \pm$ & $29.156 \pm 0.408$ & $A B a b$ & $0.258 \pm 0.031$ & $A$ ade & $34.171 \pm$ & $\mathrm{B} \mathrm{bcd}$ & $0.923 \pm 0.136$ & $\mathrm{BC}$ abcd & $0.170 \pm 0.024$ & $A B a b$ & $0.016 \pm 0.003$ & Dow & $0.113 \pm 0.018$ \\
\hline 15 & po & Tena & $0.068 \pm 0.007$ & B abcd & $0.032 \pm 0.003$ & $28.212 \pm 0.697$ & $\mathrm{~B} a b$ & $0.270 \pm 0.024$ & $A a b$ & $34.124 \pm 0.336$ & B bcd & $0.872 \pm 0.112$ & $\mathrm{C} c d$ & $0.176 \pm 0.018$ & $\mathrm{~B} a \mathrm{~b}$ & $0.017 \pm 0.002$ & $a b$ & $0.114 \pm 0.011$ \\
\hline 16 & (Amazon) & Arosemena Tola & $0.064 \pm 0.013$ & B bcd & $0.021 \pm 0.004$ & $28.169 \pm 1.001$ & $\mathrm{~B} a b$ & $0.277 \pm 0.067$ & $\mathrm{~A} \mathrm{a}$ & $34.451 \pm 1.008$ & $\mathrm{~B} \mathrm{bc}$ & $0.954 \pm 0.209$ & C abcd & $0.178 \pm 0.040$ & $\mathrm{~B} a b$ & $0.017 \pm 0.003$ & $\mathrm{~B}$ ab & $0.118 \pm 0.033$ \\
\hline 17 & & Archidona & $53 \pm 0.003$ & $\mathrm{~B}$ & $019 \pm 0.003$ & $3.654 \pm 0.433$ & $D \mathrm{du}^{2}$ & $0.239 \pm 0.043$ & A abcd & $34.184 \pm 0.712$ & $\mathrm{~B} \mathrm{bcd}$ & $0.836 \pm 0.059$ & $\mathrm{C}$ & $0.167 \pm 0.015$ & $\mathrm{~B} \mathrm{ab}$ & $0.016 \pm 0.002$ & $\mathrm{~B} \mathrm{ab}$ & $0.100 \pm 0.008$ \\
\hline
\end{tabular}

Results are expressed as mean $\pm \mathrm{SD}(n=5)$. *Different uppercase letters indicate statistically significant differences $(p<0.05)$ among provinces; different lowercase letters indicate significant differences $(p<0.05)$ among cantons. ** No significant differences $(p>0.05)$ among provinces. 
Table 4. Unsaturated Fatty Acid content ( $\mathrm{g} \mathrm{FA} / 100 \mathrm{~g}$ CF) in Nacional cocoa from the main production areas of Ecuador

\begin{tabular}{|c|c|c|c|c|c|c|c|c|c|c|}
\hline \multirow{2}{*}{$\frac{N .}{1}$} & \multirow{2}{*}{$\frac{\text { Province }}{\text { Guayas }}$} & \multirow{2}{*}{$\frac{\text { Canton }}{\text { Milagro }}$} & \multicolumn{2}{|c|}{ C16:1* } & \multicolumn{2}{|c|}{ C18:1* } & \multicolumn{2}{|c|}{ C18:2* } & \multirow{2}{*}{$\frac{\mathbf{C 1 8}^{\text {*** }}}{0.220 \pm 0.001}$} & \multirow{2}{*}{$\frac{\mathbf{C 2 0}^{\text {*** }}}{0.033 \pm 0.009}$} \\
\hline & & & $0.298 \pm 0.054$ & C bcde & $32.352 \pm 1.390$ & $A B$ abc & $2.324 \pm 0.448$ & $\mathrm{D} c d$ & & \\
\hline 2 & (Pac. Coast) & Yaguachi & $0.284 \pm 0.054$ & C colef & $32.405 \pm 1.154$ & $A B a b c$ & $2.300 \pm 0.434$ & $\mathrm{D} c d$ & $0.221 \pm 0.003$ & $0.046 \pm 0,004$ \\
\hline 3 & & Durán & $0.294 \pm 0.061$ & C bcde & $32.255 \pm 1.289$ & $\mathrm{AB} a b c$ & $2.242 \pm 0.604$ & $\mathrm{Dd}$ & $0.217 \pm 0.009$ & $0.033 \pm 0.009$ \\
\hline 4 & Los Ríos & Montalvo & $0.269 \pm 0.051$ & $B C$ def & $33.531 \pm 0.395$ & $\mathrm{~A} \mathrm{a}$ & $2.223 \pm 0.553$ & CD d & $0.216 \pm 0.013$ & $0.044 \pm 0.005$ \\
\hline 5 & (Pac. Coast) & Vinces & $0.299 \pm 0.042$ & BC bcde & $32.359 \pm 1.157$ & A abc & $2.440 \pm 0.279$ & CD bcd & $0.189 \pm 0.049$ & $0.045 \pm 0.008$ \\
\hline 6 & Manabí & Santa Ana & $0.404 \pm 0.053$ & $\mathrm{~A}$ a & $32.442 \pm 0.748$ & $\mathrm{~B} a b c$ & $2.728 \pm 0.168$ & BC abcd & $0.210 \pm 0.007$ & $0.047 \pm 0.004$ \\
\hline 7 & (Pac. Coast) & Portoviejo & $0.361 \pm 0.043$ & A ab & $31.842 \pm 0.572$ & $\mathrm{~B} b c$ & $2.626 \pm 0.207$ & BC abcd & $0.194 \pm 0.042$ & $0.041 \pm 0.004$ \\
\hline 8 & & Chone & $0.349 \pm 0.044$ & $\mathrm{~A} a b c$ & $31.937 \pm 0.358$ & $\mathrm{~B} b c$ & $2.509 \pm 0.131$ & $\mathrm{BC}$ bcd & $0.212 \pm 0.038$ & $0.040 \pm 0.008$ \\
\hline 9 & Francisco de & El Coca & $0.214 \pm 0.071$ & $\mathrm{Bf}$ & $33.131 \pm 0.600$ & $A B a b$ & $2.420 \pm 0.187$ & BCD bcd & $0.224 \pm 0.025$ & $0.040 \pm 0.011$ \\
\hline 10 & Orellana & Loreto & $0.248 \pm 0.026$ & Bef & $31.659 \pm 0.497$ & $A B C$ & $2.475 \pm 0.302$ & BCD bcd & $0.220 \pm 0.010$ & $0.040 \pm 0.006$ \\
\hline 11 & (Amazon) & Sachas & $0.296 \pm 0.065$ & B bcde & $32.366 \pm 0.215$ & $\mathrm{AB} a b c$ & $2.611 \pm 0.433$ & BCD bcd & $0.217 \pm 0.007$ & $0.052 \pm 0.013$ \\
\hline 12 & Sucumbíos & Lago Agrio & $0.330 \pm 0.052$ & C abcd & $31.924 \pm 0.699$ & $\mathrm{~B} b c$ & $2.718 \pm 0.372$ & $A B$ abcd & $0.216 \pm 0.044$ & $0.036 \pm 0.003$ \\
\hline 13 & (Amazon) & Shushufindi & $0.297 \pm 0.024$ & C bcde & $32.017 \pm 1.066$ & $\mathrm{~B} b c$ & $2.860 \pm 0.276$ & $A B a b c$ & $0.220 \pm 0.010$ & $0.039 \pm 0.005$ \\
\hline 14 & & Gonzalo Pizarro & $0.279 \pm 0.061$ & C cdf & $31.834 \pm 0.634$ & $\mathrm{~B} \mathrm{bc}$ & $2.709 \pm 0.312$ & AB abcd & $0.188 \pm 0.035$ & $0.040 \pm 0.007$ \\
\hline 15 & Napo & Tena & $0.298 \pm 0.031$ & $\mathrm{BC}$ bcde & $32.573 \pm 0.575$ & $A B a b c$ & $2.916 \pm 0.361$ & $A a b$ & $0.246 \pm 0.055$ & $0.044 \pm 0.006$ \\
\hline 16 & (Amazon) & Arosemena Tola & $0.298 \pm 0.040$ & BC bcde & $32.256 \pm 1.232$ & $A B a b c$ & $2.856 \pm 0.403$ & A abc & $0.173 \pm 0.061$ & $0.058 \pm 0.017$ \\
\hline 17 & & Archidona & $0.249 \pm 0.030$ & $\mathrm{BC}$ ef & $32.065 \pm 0.498$ & $\mathrm{AB} b \mathrm{bc}$ & $3.190 \pm 0.420$ & $\mathrm{~A} \mathrm{a}$ & $0.207 \pm 0.043$ & $0.042 \pm 0.005$ \\
\hline
\end{tabular}

Results are expressed as mean \pm SD $(n=5)$. *Different uppercase letters indicate statistically significant differences $(p<0.05)$ among provinces; different lowercase letters indicate significant differences $(p<0.05)$ among cantons. ${ }^{\star \star}$ No significant differences $(p>0.05)$ among provinces. 
Overall, these results were in agreement with the intra-country variability in the FA composition of Ecuadorian cocoa fat reported by Riaño et al. (2016). Nevertheless, it should be indicated that some FA changed while other did not change among regions and provinces, which might be a valuable information for quality control and traceability purposes.

From a nutritional point of view, the content in SFA and MUFA of Nacional cocoa beans did not change between the Pacific Coast and the Amazonian region $(p>0.05)$. The SFA content ranged between $63.56 \pm 0.50$ and $65.51 \pm 0.97 \mathrm{~g} / 100 \mathrm{~g}$ CF, while the MUFA varied from $31.95 \pm 0.48$ to $33.84 \pm 0.38 \mathrm{~g} / 100 \mathrm{~g}$ CF (Table 5). The PUFA content was significantly higher $(p<0.05)$ in the cocoa harvested in the Amazonian region than in the Pacific Coast, although mean difference between both regions was only about $0.33 \%$ of the cocoa fat $(2.63 \pm 0.36$ and $2.96 \pm 0.34 \mathrm{~g} / 100 \mathrm{~g}$ CF in Pacific Coast and Amazonian cocoas, respectively). The growing region had not an effect $(p>0.05)$ on the total amount of unsaturated FA and the ratio SFA/unsaturated FA (ratio $S / U$ ), this ratio varying from $1.75 \pm 0.04$ to $1.88 \pm 0.04$ (Table 5). A significant effect $(p<0.05)$ of the province was shown on the content of MUFA and PUFA but not for SFA and the ratio $S / U$, while a significant effect of the canton was found for all the variables (Table 5). Differences in the ratio $S / U$ attributed to the geographical origin of Ecuadorian cocoas were also reported by Riaño et al. (2016). These facts asserted that there is an effect of the environment on the profile of these FA in Ecuadorian Nacional cocoas, which may help to better select cocoas with a specific profile in FA. A good proxy for this purpose might be the ratio $S / U$, as it allows to establish the relationship between the content of the two most representative groups of $F A$ in cocoa fat. It is well known that SFA predominates over unsaturated FA in cocoa fat, which is usually considered as nutritionally negative and associated with cardiovascular diseases (Steinberg et al. 2003; Cooper et al. 2008; Mozaffarian et al. 2010; TorresMoreno et al. 2015). In this sense, the Ecuadorian cocoas having the healthiest FA profile (lowest ratio $\mathrm{S} / \mathrm{U}$ ) would belong to the cantons Montalvo (province Los Ríos), Tena (Napo), and El Coca (Francisco de Orellana), while the less desirable profile would be related to the canton Loreto (Francisco de Orellana). Nevertheless, this ratio may oversimplify the nutritional properties of cocoa beans as it takes into account stearic acid. Stearic acid (C18:0), one of the main SFA of cocoa fat, is considered a neutral fatty acid with no atherogenic and cholesterogenic effects as it is rapidly desaturated in the body and transformed into oleic acid (Steinberg et al. 2003; Cooper et al. 2008; Mozaffarian et al. 2010; Torres-Moreno et al. 2015). 
Table 5. Global Fatty Acid composition (g FA/100 g CF) in Nacional cocoa from the main production areas of Ecuador

\begin{tabular}{|c|c|c|c|c|c|c|c|c|c|c|c|c|}
\hline \multirow{2}{*}{$\frac{\text { N. }}{1}$} & \multirow{2}{*}{$\frac{\text { Province }}{\text { Guayas }}$} & \multirow{2}{*}{$\frac{\text { Canton }}{\text { Milagro }}$} & \multicolumn{2}{|c|}{ SFA } & \multicolumn{2}{|c|}{ MUFA } & \multicolumn{2}{|l|}{ PUFA } & \multicolumn{2}{|l|}{ UFA } & \multicolumn{2}{|l|}{ Ratio S/U } \\
\hline & & & $64.53 \pm 1.19$ & A abc & $32.68 \pm 1.35$ & $A B a b c$ & $2.54 \pm 0.45$ & D cde & $35.23 \pm 1.22$ & $\mathrm{~B} a b$ & $1.84 \pm 0.09$ & $\mathrm{~A} a b$ \\
\hline 2 & (Pac. Coast) & Yaguachi & $65.51 \pm 0.97$ & A $a b c$ & $32.73 \pm 1.10$ & $A B a b c$ & $2.52 \pm 0.44$ & D cde & $35.22 \pm 0.99$ & $\mathrm{~B} a b$ & $1.83 \pm 0.08$ & $\mathrm{~A} a \mathrm{~b}$ \\
\hline 3 & & Durán & $64.75 \pm 0.87$ & $\mathrm{~A} a b c$ & $32.58 \pm 1.24$ & $A B$ abc & $2.46 \pm 0.61$ & $\mathrm{D}$ de & $35.04 \pm 0.88$ & $\mathrm{~B} a b$ & $1.85 \pm 0.07$ & $\mathrm{Aab}$ \\
\hline 4 & Los Ríos & Montalvo & $63.56 \pm 0.50$ & $A C$ & $33.84 \pm 0.38$ & $\mathrm{~A}$ a & $2.44 \pm 0.55$ & CD e & $36.28 \pm 0.44$ & $A B a$ & $1.75 \pm 0.04$ & $A b$ \\
\hline 5 & (Pac. Coast) & Vinces & $64.53 \pm 1.01$ & A abc & $32.70 \pm 1.12$ & $\mathrm{~A} a b c$ & $2.63 \pm 0.27$ & CD bcde & $35.33 \pm 1.00$ & $\mathrm{AB} a b$ & $1.83 \pm 0.07$ & $\mathrm{Aab}$ \\
\hline 6 & Manabí & Santa Ana & $64.10 \pm 0.62$ & A $a b c$ & $32.89 \pm 0.72$ & $\mathrm{~B} a b c$ & $2.94 \pm 0.17$ & BC abcde & $35.83 \pm 0.60$ & $\mathrm{AB} a b$ & $1.79 \pm 0.05$ & $\mathrm{~A} a \mathrm{~b}$ \\
\hline 7 & (Pac. Coast) & Portoviejo & $64.90 \pm 0.63$ & $A a b$ & $32.24 \pm 0.54$ & $\mathrm{~B} b \mathrm{bc}$ & $2.82 \pm 0.17$ & BC abcde & $35.06 \pm 0.61$ & $A B a b$ & $1.85 \pm 0.05$ & $\mathrm{~A} a b$ \\
\hline 8 & & Chone & $64.88 \pm 0.42$ & $\mathrm{~A} a b$ & $32.83 \pm 0.36$ & $\mathrm{~B} \mathrm{bc}$ & $2.72 \pm 0.14$ & $\mathrm{BC}$ bcde & $35.05 \pm 0.43$ & $\mathrm{AB} a$ & $1.85 \pm 0.02$ & $\mathrm{~A} \mathrm{ab}$ \\
\hline 9 & Francisco de & El Coca & $63.72 \pm 0.64$ & $\mathrm{~A} b \mathrm{~b}$ & $33.38 \pm 0.62$ & $A B a b$ & $2.64 \pm 0.20$ & BCD bcde & $36.03 \pm 0.61$ & $A B a$ & $1.77 \pm 0.05$ & $\mathrm{Ab}$ \\
\hline 10 & Orellana & Loreto & $65.11 \pm 0.51$ & $\mathrm{~A} \mathrm{a}$ & $31.95 \pm 0.48$ & $A B C$ & $2.69 \pm 0.30$ & BCD bcde & $34.64 \pm 0.52$ & $A B b$ & $1.88 \pm 0.04$ & $\mathrm{~A} \mathrm{a}$ \\
\hline 11 & (Amazon) & Sachas & $64.19 \pm 0.61$ & $\mathrm{~A} a b c$ & $32.71 \pm 0.78$ & $A B a b c$ & $2.83 \pm 0.43$ & BCD abcde & $35.54 \pm 0.61$ & $\mathrm{AB} a b$ & $1.81 \pm 0.04$ & $\mathrm{Aab}$ \\
\hline 12 & Sucumbíos & Lago Agrio & $64.52 \pm 0.67$ & A abc & $32.29 \pm 0.70$ & $\mathrm{~B} a b c$ & $2.93 \pm 0.38$ & AB abcde & $35.22 \pm 0.67$ & $A B a b$ & $1.83 \pm 0.05$ & $\mathrm{~A} a b$ \\
\hline 13 & (Amazon) & Shushufindi & $64.37 \pm 1.24$ & A $a b c$ & $32.35 \pm 1.06$ & $\mathrm{~B} b \mathrm{bc}$ & $3.08 \pm 0.28$ & $A B a b c$ & $35.43 \pm 1.23$ & $\mathrm{AB} a b$ & $1.82 \pm 0.10$ & $\mathrm{~A} a \mathrm{~b}$ \\
\hline 14 & & Gonzalo Pizarro & $64.89 \pm 0.70$ & A abc & $32.15 \pm 0.61$ & $\mathrm{~B} \mathrm{bc}$ & $2.90 \pm 0.33$ & AB abcde & $35.05 \pm 0.64$ & $\mathrm{AB} a b$ & $1.85 \pm 0.05$ & $\mathrm{~A} a b$ \\
\hline 15 & Napo & Tena & $64.89 \pm 0.81$ & A $a b c$ & $32.92 \pm 0.60$ & $A B a b c$ & $3.16 \pm 0.41$ & $A a b$ & $36.08 \pm 0.81$ & $\mathrm{AB}$ a & $1.77 \pm 0.07$ & $\mathrm{Ab}$ \\
\hline 16 & (Amazon) & Arosemena Tola & $64.25 \pm 1.35$ & A abc & $32.61 \pm 1.21$ & $A B a b c$ & $3.03 \pm 0.46$ & A abcd & $35.64 \pm 1.32$ & $A B a b$ & $1.83 \pm 0.08$ & $A a b$ \\
\hline 17 & & Archidona & $64.27 \pm 0.83$ & $\mathrm{~A} a b c$ & $32.36 \pm 0.50$ & $\mathrm{AB} b c$ & $3.40 \pm 0.42$ & $\mathrm{~A} \mathrm{a}$ & $35.75 \pm 0.86$ & $\mathrm{AB} a b$ & $1.83 \pm 0.08$ & $\mathrm{~A} \mathrm{ab}$ \\
\hline
\end{tabular}

Results are expressed as mean $\pm \mathrm{SD}(n=5)$. ${ }^{*}$ Different uppercase letters indicate statistically significant differences $(p<0.05)$ among provinces; different lowercase letters indicate significant differences $(p<0.05)$ among cantons. SFA, saturated fatty acids; MUFA, monounsaturated fatty acids; PUFA, polyunsaturated fatty acids; UFA, unsaturated fatty acids; ratio $S / U$, saturated/unsaturated fatty acid ratio. 
Therefore, the selection of a certain profile of FA rather than focusing on a single ratio should be pursued when choosing cocoas for the development of chocolates with a healthier FA profile. Some insights have been proposed by Mustiga et al. (2019), who indicated that palmitic acid amounts and the sum of stearic, oleic, and linoleic acids were negatively correlated. This fact was also shown by an ad-hoc Pearson's correlation $(r=-0.956, p<0.01)$ herein, so this relationship may represent a further tool for the selection of cocoas with a low palmitic acid content and high amounts of stearic and unsaturated FA.

\subsection{Multivariate analysis}

Principal component analysis (PCA) was used to provide a complete picture of the lipid content and profile of Nacional cocoa samples grown in Ecuador, as well as the relationships among different fatty acids. This led to a comprehensive analysis of the influence of the growing area on the cocoa fat composition. Five PCs accounted for $73.5 \%$ of the total variability when total fat and all the fatty acids were included in the analysis to get descriptive insights in cocoa fat composition. The first principal component (PC1) explained up to $21.9 \%$ of the total variance and was positively associated with C22:0, C20:0, C24:0, and C23:0. PC2 (18.2\% total variance) was negatively linked to $C 16: 0$, while it was positively related to $C 18: 0, C 16: 1$, and $C 14: 0$. PC3 (14.6\%) had positive loads for total fat, C17:0, C18:2, and C15:0, PC4 (9.7\%) was related positively to $\mathrm{C} 18: 3$ and negatively to $\mathrm{C} 20: 1$, and PC5 $(9.1 \%)$ was loaded by C18:1. Individual scores respect to each PC confirmed the influence of the geographic differences on the fat composition. Cocoa samples from the Pacific Coast had higher PC1 and PC2 scores and lower PC3 scores than the samples grown in the Amazonian region, samples from both growing areas being well differentiated. Accordingly, cocoas grown in the Pacific Coast cantons presented a lower fat content, higher amounts of C14:0, C18:0, C20:0, C22:0, C23:0 C24:0, and C16:1, and lower proportions of C15:0, $\mathrm{C} 16: 0, \mathrm{C} 17: 0$, and C18:2 than Amazonian cocoas. Samples coming from some provinces also grouped well according to their scores for specific PCs; for instance, cocoa samples from Manabí province presented high PC2 scores. Clear clusters related to specific cantons were not observed.

A second PCA was carried out to better understand the impact of the growing area in main cocoa parameters of nutritional interest. For that purpose, the fat content and the main fatty acids defining cocoa fat profile (C16:0, C18:0, C18:1, C18:2, and C20:0) were included in the analysis. 


\subsubsection{Relationship among all the fatty acids and production areas}

Principal component analysis (PCA) was used to provide a comprehensive picture of the lipid composition of Nacional cocoa samples grown in Ecuador, as well as the relationships among different fatty acids. Five PCs accounted for $73.5 \%$ of the total variability when total fat and all the fatty acids were included in the analysis to get descriptive insights in cocoa fat composition. The first principal component (PC1) explained up to $21.9 \%$ of the total variance and was positively associated with C22:0, C20:0, C24:0, and C23:0. PC2 (18.2\% total variance) was negatively linked to C16:0, while it was positively related to C18:0, C16:1, and C14:0. PC3 (14.6\%) had positive loads for total fat, $\mathrm{C} 17: 0, \mathrm{C} 18: 2$, and C15:0, PC4 (9.7\%) was related positively to $\mathrm{C} 18: 3$ and negatively to C20:1, and PC5 (9.1\%) was loaded by C18:1. Individual scores for each PC confirmed the influence of the geographic differences on the cocoa fat composition. Cocoa samples from the Pacific Coast had higher PC1 and PC2 scores and lower PC3 scores than the samples grown in the Amazonian region, samples from both growing areas being well differentiated. Accordingly, cocoas grown in the Pacific Coast cantons presented a lower fat content, higher amounts of C14:0, C18:0, C20:0, C22:0, C23:0 C24:0, and C16:1, and lower proportions of C15:0, C16:0, C17:0, and C18:2 than Amazonian cocoas. Samples coming from some provinces also grouped well according to their scores for specific PCs; for instance, cocoa samples from Manabí province presented high PC2 scores. Clear clusters related to specific cantons were however not observed.

To date, there is no much information on the main factors conditioning the FA composition of cocoa beans. Although it has been reported that the genotypic characteristics, the growing location, the environmental conditions and the post-harvest treatments may condition the FA profile (D W Lehrian et al. 1980; Jahurul et al. 2013; Zyzelewicz et al. 2014; Servent et al. 2018; Mustiga et al. 2019), mechanistic studies are lacking. Recently, a study by Mustiga et al. (2019), indicated that, besides being conditioned by specific allelic differences, palmitic acid levels are directly correlated to the temperature. This could not be confirmed from our data since temperatures for each farm were not evaluated. Nonetheless, slightly lower averages temperatures were found in the most of the Amazonian provinces, where higher levels of palmitic acid were recorded. 


\subsubsection{Relationship among main fatty acids of nutritional interest and production areas}

A second PCA was carried out to better understand the impact of the growing area in main cocoa parameters of nutritional interest. For that purpose, only the fat content and the main fatty acids defining cocoa fat profile (C16:0, C18:0, C18:1, C18:2, and C20:0) were included in the analysis. Three PCs explained up to $81.6 \%$ of the total variation, with each PC accounting for a similar variation (PC1, PC2, and PC3 explained $29.1 \%$, $26.7 \%$ and $25.8 \%$, respectively). PC1 had negative load for $\mathrm{C} 16: 0$ and positive load for C18:0, PC2 was positively associated to total fat and negatively to C20:0, and PC3 had negative loadings for $\mathrm{C} 18: 1$ and positive ones for C18:2 (Figure 2A and 2C). These loadings provided robust indications of the inverse relationship between couples of main cocoa fatty acids. Regarding individual scores (Figure 2B and 2D), cocoa samples from the Amazonian region presented positive PC2 scores while Pacific Coast samples had negative PC2 scores $(p<0.001)$, pointing out the higher total fat content of Amazonian cocoas. Scores for PC1 and PC3 did not serve to group samples according to the growing region $(p>0.05)$, but they served to characterize the samples coming from specific provinces (Figure 2). For instance, most of the samples grown in the Amazonian province Napo and Pacific Coast province Manabí had positive PC1 scores (low C16:0, high C18:0), while Amazonian provinces Francisco de Orellana and Sucumbíos and Pacific Coast provinces Guayas and Los Ríos had negative PC1 scores (high C16:0, low C18:0) (Table 4). Sucumbíos province also had positive scores for PC3 (Figure 2D), accounting for higher values of C18:2 and lower C18:1 (Figure 2). Similary, scores for PC2 served to discriminate samples from the Amazonian province Napo (highest PC2 scores) and from the Pacific Coast province Guayas (lowest PC2 scores) (Figure 2). Some cantons from both regions presented a high variability in their scores for PC1 (i.e., Santa Ana and Portoviejo) or PC2 (i.e., Milagro, Chone, and Arosemena Tola), contrary to most of the cantons which grouped quite well for these PCs (Figure 2B). PC3 also served to characterize some cantons according to the predominance of a specific unsaturated $\mathrm{C} 18$ (C18:1 or C18:2). In this sense, cantons Portoviejo, Loreto, Shushufindi, and Gonzalo Pizarro had significant positive scores for PC3 (low C18:1, high C18:2) with respect to the rest of cantons, while Montalvo significant exhibited negative PC3 scores (high C18:1, low C18:2) (Figure 2). In general, this simplified PCA focused on main fatty acids of nutritional interest served to define the characteristics of each sample attending to three different geographical levels, which may be useful for the selection of raw materials with a specific lipid content and profile. 


\begin{tabular}{lll}
\multicolumn{2}{c}{ Province } \\
PC_Guayas & AM_Fr.Orellana & \\
PC_Los Ríos & AM_Sucumbíos & D \\
PC_Manabí & AM_Napo & \\
& & \\
C & &
\end{tabular}

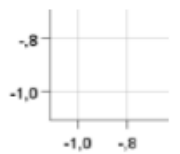

Figure 2. Principal component analysis of the lipid profile of Ecuadorian Nacional cocoas. A) loading plot of PC1 versus PC2; B) score plot and distribution of the samples in the consensus space for PC1 and PC2; C) loading plot of PC1 versus PC3; D) score plot and distribution of the samples for PC1 and PC3. In the loading plots: C16:0, palmitic acid; C18:0, stearic acid; C18:1, oleic acid; C18:2, linoleic acid; C20:0, arachidic acid; and TF, total fat. In the score plots, circles corresponded to cantons from the Amazonian region (AM), while double triangles accounted for cantons from the Pacific Coast (PC). Cantons from each province are abbreviated as reported in Table 1.

This data should be considered only for Ecuadorian cocoas unless they replicated in other experimental sets at national level using the same variety. Contrary to this work, a PCA carried out by Servent et al. (2018), for cocoas from Ecuador, Madagascar, and 
Dominican Republic showed a direct correlation between C18:1 and C18:2, as well as a lack of correlation between $\mathrm{C} 16: 0$ and $\mathrm{C} 18: 0$. In this case, other parameters were considered for the PCA and different varieties (Criollo and Trinitario) taken into account. Nevertheless, an inverse correlation between palmitic acid and stearic acid was shown by Mustiga et al. (2019), after a massive profiling of cocoa samples, in agreement with our data and shedding light on the relationship among main cocoa FA.

\section{Conclusions}

The lipid fraction of Nacional cocoa beans from Ecuador was characterised for the first time, reporting ranges useful for the definition of their fat profile. Information on the impact of the growing area on the cocoa fat composition was provided, highlighting a notable effect of the region and the province on the definition of the total fat content and FA composition of Ecuadorian cocoas. Although cantons within the same province shared common characteristics, like the fat content, the variation in the FA profile among farms within each canton made difficult to group samples according to the cantons. The differences among geographical areas represent a helpful tool for producers and the industry to boost the competitive development of the cocoa production chain in Ecuador. They could be used to make a more accurate selection of cocoa beans taken into account both technological and nutritional characteristics of the cocoa butter. Further research should be conducted on the chocolate or other confectionary products that may be prepared by using cocoa beans with different FA compositions, assessing also whether small differences in the FA profile may have an impact at nutritional level.

\section{Disclosure statement}

The authors declare no conflict of interest.

\section{Funding}

The authors thank INIAP and SENECYT for funding this research through the Fortalecimiento and PIC-12-INIAP-003 projects, respectively.

\section{References}

Afoakwa EO, Paterson A, Fowler M, Ryan A. 2008. Flavor formation and character in cocoa and chocolate : A critical review. Crit Rev Food Sci Nutr. 48(9):840-857.

Afoakwa EO, Quao J, Simpson Budu A, Takrama J, Kwesi Saalia F. 2011. Effect of pulp preconditioning on acidification, proteolysis, sugars and free fatty acids 
concentration during fermentation of cocoa (Theobroma cacao) beans. Int $\mathrm{J}$ Food Sci Nutr. 62(7):755-764.

Belščak A, Komes D, Horžić D, Ganić K, Karlović D. 2009. Comparative study of commercially available cocoa products in terms of their bioactive composition. Food Res Int. 42:707-716.

Brunetto M del R, Gutiérrez L, Delgado Y, Gallignani M, Zambrano A, Gómez Á, Ramos G, Romero C. 2007. Determination of theobromine, theophylline and caffeine in cocoa samples by a high- performance liquid ... Determination of theobromine, theophylline and caffeine in method with on-line sample cleanup in a switching-column system. Food Chem. 100(June):459-467.

Caligiani A, Palla L, Acquotti D, Marseglia A, Palla G. 2014. Application of 1H NMR for the characterisation of cocoa beans of different geographical origins and fermentation levels. Food Chem. 157:94-99.

CAOBISCO, ECA, FCC. 2015. Cocoa Beans: Chocolate and Cocoa Industry Quality Requirements. Brussels, Belgium. http://www.cocoaquality.eu

Caprioli G, Fiorini D, Maggi F, Nicoletti M, Ricciutelli M, Toniolo C, Prosper B, Vittori S, Sagratini G. 2016. Nutritional composition, bioactive compounds and volatile profile of cocoa beans from different regions of Cameroon. Int $\mathrm{J}$ Food Sci Nutr. 67(4):422-430.

Carrillo LC, Londoño-Londoño J, Gil A. 2014. Comparison of polyphenol, methylxanthines and antioxidant activity in Theobroma cacao beans from different cocoa-growing areas in Colombia. Food Res Int. 60:273-280.

Cooper KA, Donovan JL, Waterhouse AI, Williamson G. 2008. Cocoa and health: A decade of research. Br J Nutr. 99(1):1-11.

Dand R. 2011a. History and origins of the international cocoa trade. In: Int Cocoa Trade. Third edit. Cambridge, United Kingdom: Woodhead Publishing; p. 1-22.

Dand R. 2011b. Agronomics of international cocoa production. In: Int Cocoa Trade. Third edit. Cambridge, United Kingdom: Woodhead Publishing Limited; p. 23-64.

Espín S, Samaniego I. 2016. Manual para análisis de parámetros químicos asociados a la calidad del cacao. Quito, Ecuador.

Exporters-Ecuador National Association of Cocoa. 2016. Sabor Arriba. Guayaquil, Ecuador.

Foubert I, Vanrolleghem PA, Thas O, Dewettinck K. 2004. Influence of Chemical 
Composition on the Isothermal Cocoa Butter Crystallization. Food Eng Phys Prop. 69(9):471-477.

Hernandez B, Castellote AI, Permanyer JJ. 1991. Triglyceride analysis of cocoa beans from different geographical origins. Food Chem. 41(3):269-276.

International Cocoa Organization. 2017. Annual Report 2014/2015. Abiyán, Republic of Cote d'Ivoire.

Jahurul MHA, Zaidul ISM, Norulaini NAN, Sahena F, Jinap S, Azmir J, Sharif KM, Mohd Omar AK. 2013. Cocoa butter fats and possibilities of substitution in food products concerning cocoa varieties, alternative sources, extraction methods, composition, and characteristics. J Food Eng. 117(4):467-476.

Jimenez, J., Amores, F., \& Solórzano E. 2014. Componentes de identidad para reconocer las diferencias del cacao que se produce en varias regiones del Ecuador. Quevedo, Ecuador.

Jimenez, J., Amores, F., \& Solórzano E, Jiménez J, Amores F, Solórzano E, Jimenez, J., Amores, F., \& Solórzano E, Jiménez J, Amores F, Solórzano E. 2014. Componentes de identidad para reconocer las diferencias del cacao que se produce en varias regiones del Ecuador. Estación Experimental Tropical Pichilingue. INIAP. Boletín Técnico : 164. Quevedo, Ecuador, Ecuador.

Kongor JE, Hinneh M, de Walle D Van, Afoakwa EO, Boeckx P, Dewettinck K. 2016. Factors influencing quality variation in cocoa ( Theobroma cacao ) bean flavour profile - A review. Food Res Int. 82(January):44-52.

Lehrian D. W., Keeney PG, Butler DR. 1980. Triglyceride characteristics of cocoa butter from cacao fruit matured in a microclimate of elevated temperature1. J Am Oil Chem Soc. 57(2):66-69.

Lehrian D W, Keeney PG, Science F. 1980. Triglyceride Characteristics of Cocoa Butter from Cacao Fruit Matured in a Microclimate of Elevated Temperature I. (February):66-67.

Ministry of Agriculture and Livestock. 2016. Reportes Dinámicos ESPAC. In Superficie, Producción y Rendimiento (2002-2016) (Vol. 2017). Quito, Ecuador.

Motamayor JC, Lachenaud P, Wallace da Silva J, Loor R, Kuhn D, Brown S, Schnell R. 2008. Geographic and genetic population differentiation of the Amazonian chocolate tree ( Theobroma cacao L ) Geographic and Genetic Population Differentiation of the Amazonian Chocolate Tree ( Theobroma cacao L ). PLoS 
One. 3(10).

Mozaffarian D, Micha R, Wallace S. 2010. Effects on coronary heart disease of increasing polyunsaturated fat in place of saturated fat: A systematic review and meta-analysis of randomized controlled trials. PLoS Med. 7(3).

Mustiga GM, Morrissey J, Stack JC, DuVal A, Royaert S, Jansen J, Bizzotto C, VillelaDias C, Mei L, Cahoon EB, et al. 2019. Identification of Climate and Genetic Factors That Control Fat Content and Fatty Acid Composition of Theobroma cacao L. Beans. Front Plant Sci. 10(October):1-20.

Naik B, Kumar V. 2014. Cocoa Butter and Its Alternatives: A Reveiw. J Bioresour Eng Technol. 1(March 2014):7-17.

Oracz J, Nebesny E. 2014. Influence of roasting conditions on the biogenic amine content in cocoa beans of different Theobroma cacao cultivars. Food Res Int. $55: 1-10$.

Parra O, Gallego AM, Urrea A, Rojas LF, Correa C, Atehortúa L. 2017. Biochemical precursor effects on the fatty acid production in cell suspension cultures of Theobroma cacao L. Plant Physiol Biochem. 111:59-66.

Pontillon J. 1997. Cacao et chocolat: Production, utilisation, caractéristiques. Paris, France: Tec et doc-Lavoisier.

Riaño N, Chica M, Echeverri L, Aguirre J, Ortiz A, Pineda R, Olarte N. 2016. Contenido de grasa total, perfil de Ácidos grasos y triglicéridos proveniente de cacaos finos de aroma: Colombia, Ecuador, Perú, Venezuela. Vitae. 23(April):S226-S230.

Samaniego I, Espín S, Quiroz J, Ortiz B, Carrillo W, García-Viguera C, Mena P. 2020. Effect of the growing area on the methylxanthines and flavan-3-ols content in cocoa beans from Ecuador. J ofFood Compos Anal. 1(January):103448.

Servent A, Boulanger R, Davrieux F, Pinot MN, Tardan E, Forestier-Chiron N, Hue C. 2018. Assessment of cocoa (Theobroma cacao L.) butter content and composition throughout fermentations. Food Res Int. 107(2017):675-682.

Steinberg FM, Bearden MM, Keen CL. 2003. Cocoa and chocolate flavonoids: implications for cardiovascular health. J Am Diet Assoc. 103(2):215.

De Taeye C, Bodart M, Caullet G, Collin S. 2017. Roasting conditions for preserving cocoa flavan-3-ol monomers and oligomers: interesting behaviour of Criollo clones. J Sci Food Agric. 97(12):4001-4008.

Tezara W, De Almeida J, Valencia E, Cortes JL, Bolaños MJ. 2015. Actividad 
fotoquímica de clones élite de cacao (Theobroma cacao L.) en el norte de la provincia de Esmeraldas. Investig y Saberes. 4(3):35-72.

Torres-Moreno M, Torrescasana E, Salas-Salvadó J, Blanch C, Torres-Moreno M, Torrescasana E, Salas-Salvadó J, Blanch C. 2015. Nutritional composition and fatty acids profile in cocoa beans and chocolates with different geographical origin and processing conditions. Food Chem. 166:125-132.

Vargas Jentzsch P, Ciobotă V, Salinas W, Kampe B, Aponte M, Rösch P, Popp J, Ramos LA, Aponte PM, Vargas P, et al. 2016. Distinction of Ecuadorian varieties of fermented cocoa beans using Raman spectroscopy. Food Chem. 211:274-280.

Wood GAR, Lass RA. 1985. Cocoa. 4th Editio. Longman, editor. London, United Kingdom.

Zyzelewicz D, Budryn G, Krysiak W, Oracz J, Nebesny E, Bojczuk M, Dorota Ż, Oracz J, Nebesny E, Zyzelewicz D, et al. 2014. Influence of roasting conditions on fatty acid composition and oxidative changes of cocoa butter extracted from cocoa bean of Forastero variety cultivated in Togo. Food Res Int. 63:328-343.

Żyżelewicz D, Budryn G, Oracz J, Antolak H, Kręgiel D, Kaczmarska M. 2018. The effect on bioactive components and characteristics of chocolate by functionalization with raw cocoa beans. Food Res Int. 113(March):234-244. 


\title{
Capítulo IV
}

\author{
Discusión \\ General de \\ Resultados
}


El cacao producido en el Ecuador posee características sensoriales particulares, que lo hacen único y especial; sobresaliendo en el mercado internacional con su reconocido sabor "Arriba". Estos detalles de sabor y aroma están determinados por el origen genético, y se logran con el correcto tratamiento poscosecha; sumado a condiciones naturales de producción (luminosidad, suelo, clima, temperatura, etc.) típicas de la región Litoral (Costa del Pacífico) y Amazónica del Ecuador (PROECUADOR, 2013).

En la actualidad, la evaluación de la calidad del cacao Nacional ecuatoriano, se establece mediante parámetros físicos y pruebas organolépticas, las mismas que son subjetivas. Por lo cual, en esta investigación se ha propuesto; buscar parámetros objetivos de calidad, en base a la composición bioquímica de los granos de cacao, lo cual permitirá contribuir en la certificación de origen de este tipo especial de cacao producido en el Ecuador.

En este contexto, la presente tesis doctoral se enfocó en evaluar los principales componentes químicos no volátiles en granos de cacao Nacional, procedentes de las principales zonas productoras de la región Litoral (Costa del Pacífico) y región Amazónica del Ecuador. Este trabajo se realizó en dos etapas; en la primera etapa (publicación 1) se evaluó el contenido de metilxantinas (Teobromina y Cafeína), compuestos polifenólicos totales y su perfil de flavan-3-oles ((+)-catequina, (-)epicatequina y procianidinas dímeros (B2) y trímeros (C1)), en la segunda etapa (publicación 2) se determinó el contenido total de grasa y el perfil de ácidos grasos, en muestras de cacao fermentadas (96 horas de fermentación) y secas colectadas en 85 fincas de productores de 17 cantones del Ecuador.

Este trabajo de investigación permitió caracterizar el $65 \%$ de la composición bioquímica del grano de cacao ecuatoriano (fracción lipídica 50 al 55\%, metilxantinas 2 al $3 \%$ y compuestos polifenólicos 5 al 10\% aproximadamente), así como el efecto de la zona geográfica de producción sobre el contenido de estos fitoquímicos, constituyendo una información inédita para el sector cacaotero del Ecuador y una herramienta de calidad, que será considerada a corto plazo para establecer nuevos requisitos para certificación del origen del cacao ecuatoriano. 


\subsection{Metilxantinas y compuestos fenólicos en cacao "Nacional" ecuatoriano. (Resultados relevantes de la Publicación 1).}

\subsubsection{Contenido de Metilxantinas}

En la presente tesis doctoral uno de los objetivos planteados, fue la identificación y cuantificación del contenido de metilxantinas; teobromina y cafeína, debido a que en los últimos años se ha evidenciado que la relación entre el contenido de teobromina sobre el contenido de cafeína (TBR/CAF), permite inferir en el origen genético y geográfico del cacao, constituyendo un importante parámetro de calidad para diferenciar cacaos finos de ordinarios.

Actualmente, a nivel mundial se definen tres grupos genéticos de cacao: El Criollo, el Forastero y el Trinitario en función de las características de la flor, los frutos y los granos. Los valores de la relación (TBR/CAF), permiten clasificar a los diferentes materiales de cacao, así se ha descrito que los cacaos Criollos presentan una relación (TBR/CAF) de 2 a 3, para cacao de tipo Trinitario estos valores van de 3 a 10 y en cacao Forastero de 10 en adelante. Por otra parte, desde el punto de vista comercial, los cacaos de origen Criollo y Trinitario son considerados como Finos (Brunetto et al., 2007; Diomande et al., 2015; Espín et al., 2007; Loureiro et al., 2017).

Los resultados obtenidos en este estudio, permitieron establecer que el cacao procedente de la región Amazónica, presentó contenidos de cafeína que variaron en el rango de $0,32 \pm 0.06$ a $0.43 \pm 0.11 \mathrm{~g} / 100 \mathrm{~g}$ DW y teobromina entre $2.04 \pm 0.15$ a $2.56 \pm$ $0.56 \mathrm{~g} / 100 \mathrm{~g} \mathrm{DW}$, obteniéndose una relación TBR/CAF entre 4.81 a 6.64; que los clasifica como cacaos del grupo Trinitario, demostrándose que los materiales producidos en esta región son cacaos Finos.

Para el caso de las muestras que corresponden a la región Litoral, se obtuvieron valores de cafeína en el rango de $0.20 \pm 0.05$ a $0.33 \pm 0.09 \mathrm{~g} / 100 \mathrm{~g}$ DW y de teobromina de $1.52 \pm 0.21$ a $1.85 \pm 0.35 \mathrm{~g} / 100 \mathrm{~g} \mathrm{DW}$, con una relación TBR/CAF que varió entre 5.42 a 7.60, lo que permitió clasificar al cacao de esta región dentro del grupo Trinitario, demostrándose de igual manera que son cacaos Finos.

Actualmente en el Ecuador existen pocos materiales de cacao "Nacional", la mayoría de materiales son híbridos de este tipo especial de cacao con materiales tipo 
Trinitarios introducidos de Venezuela y Trinidad y Tobago, lo que se conoce como "Complejo Nacional x Trinitario". Los resultados obtenidos mediante la relación TBR/CAF, confirman este origen genético del cacao ecuatoriano (Espín et al., 2007; Samaniego et al., 2020). Adicionalmente, se demostró mediante el análisis estadístico de los resultados, que esta relación no se ve afectada por la zona geográfica de producción ( $p>0,05$ ); lo que confirma que sus valores están directamente relacionados con el origen genético de los materiales de cacao. Por lo tanto, constituye una herramienta muy promisoria para definir el origen genético del cacao e inferir sobre la calidad y su precio en el mercado.

\subsubsection{Contenido de compuestos fenólicos}

El contenido de compuestos fenólicos, constituye un parámetro bioquímico importante que permite definir la calidad del cacao, debido a que este grupo de fitoquímicos están relacionados con ciertas propiedades organolépticas responsables del color violeta de los granos y de su sabor astringente. Además, de sus propiedades nutracéuticas, mismas que reducen el riesgo de contraer enfermedades crónicas, gracias a sus propiedades antioxidantes (Aprotosoaie et al., 2016; Wollgast \& Anklam, 2000).

La caracterización del contenido total de compuestos fenólicos realizada en cacao Nacional ecuatoriano, demostró que el contenido promedio por provincia varió en el rango de $33,55 \pm 5.74$ a $47.40 \pm 6.20 \mathrm{mg}$ de GAE/g DW; en muestras procedentes de la región Litoral. Mientras que, en materiales procedentes de la región Amazónica, los contenidos promedios por provincia variaron en el rango de $42.75 \pm 8.19$ a $71.66 \pm$ $3.94 \mathrm{mg}$ de GAE/g DW (Figura 15). Estos contenidos de TPC se encuentran dentro de los valores referenciales obtenidos por otros investigadores, los mismos indican que; el cacao fermentado presenta un contenido de compuestos fenólicos totales en el rango de 33 a $100 \mathrm{mg} / \mathrm{g}$ DW, valores superiores a $100 \mathrm{mg} / \mathrm{g}$ DW indican un proceso de fermentación deficiente. Esto influye significativamente en la calidad de los granos; puesto que, durante este proceso poscosecha, los compuestos fenólicos se someten a reacciones de condensación oxidativa, las cuales conllevan al cambio de coloración de violeta a marrón (color chocolate característico). A nivel sensorial, estos procesos disminuyen la astringencia de los granos y permiten la formación de precursores de sabor y aroma (Aprotosoaie et al., 2016; Hue et al., 2014; Wollgast \& Anklam, 2000). 
El análisis estadístico de los resultados, demostró que existe un efecto de la zona geográfica de producción sobre el contenido de TPC $(p<0.05)$, confirmando los estudios realizados por otros investigadores (de Souza et al., 2018; Kim et al., 2011 Ribeiro et al., 2019; Wollgast \& Anklam, 2000). A partir de esta información, se identificó las zonas de producción que generan cacao Fino con alto contenido de TPC, lo cual constituye un parámetro de calidad que agrega valor a las almendras de estas zonas y abren nuevos nichos de mercado, para comercializar cacao ecuatoriano con alto contenido de compuestos bioactivos.

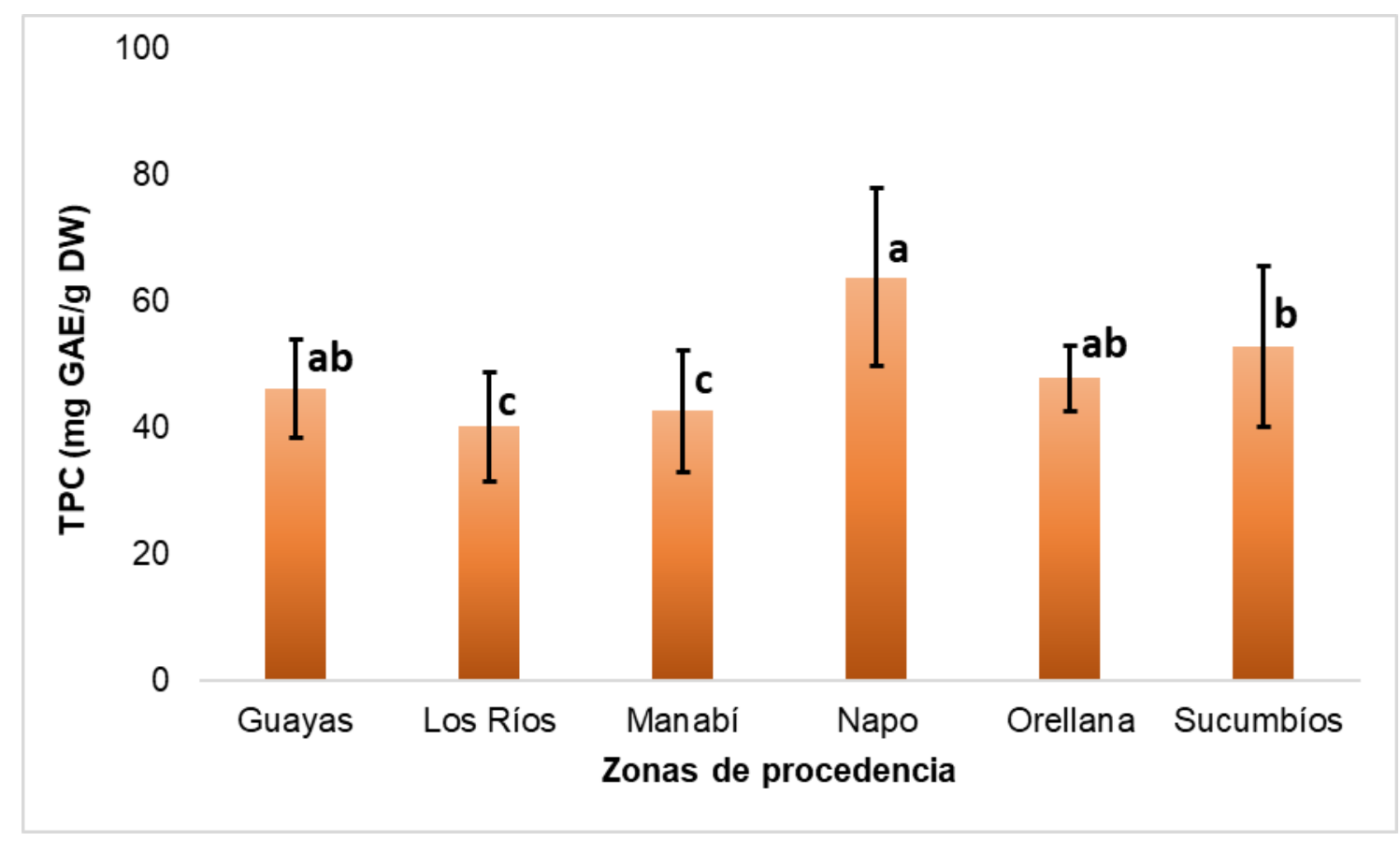

Figura 15. Contenido promedio por provincia de TPC en cacao Nacional ecuatoriano. Resultados expresados como la media \pm SD $(n=15)$. Diferentes letras indican diferencias estadísticamente significativas $(P<0.05)$ entre provincias.

\subsubsection{Contenido de Flavan-3-ols monómeros y procianidinas.}

Estudios previos realizados por varios investigadores, han reportado que los diferentes tipos de compuestos fenólicos identificados en las almendras de cacao o en los productos derivados del cacao son: catequinas o flavan-3-oles (37\%), procianidinas (58\%), antocianinas (4\%), flavonoles glicosidados (1\%) (Castro et al., 2020; de Souza et al., 2018; Gutiérrez Maydata, 2002; Kim et al., 2011; Ribeiro et al., 2019). En función de esta información, se evaluó el contenido de los principales compuestos fenólicos; monómeros (-)-epicatequina, (+)-catequina y los polímeros procianidina $\mathrm{B} 2$ y $\mathrm{C} 1$, en 
muestras de cacao Nacional procedente de las principales zonas productoras de la región Amazónica y Litoral del Ecuador.

Los resultados obtenidos en este estudio, demostraron que el perfil de compuestos fenólicos en cacao Nacional ecuatoriano presenta mayores contenidos de (-)epicatequina (3.45 \pm 1.13 a $13.16 \pm 3.61 \mathrm{mg} \mathrm{EPI} / \mathrm{g} \mathrm{DW})$, seguido de (+)-catequina $(4.20 \pm 0.86$ a $8.93 \pm 4.94 \mathrm{mg}$ CAT $/ \mathrm{g}$ DW $)$ y Procianidina B2 $(2.13 \pm 0.50$ a $7.85 \pm 3.24$ mg PC-B2/ g DW) y menores concentraciones de procianidina C1 (2.53 \pm 0.51 a $6.52 \pm$ $1.55 \mathrm{mg} \mathrm{PC}-\mathrm{C} 1 / \mathrm{g}$ DW) (Figura 16). Estos resultados son comparables con los reportados por Wollgast \& Anklam (2000), quien reportó que el cacao presenta altos contenidos de (-)-epicatequina y menores contenidos de (+)-catequina y procianidinas.

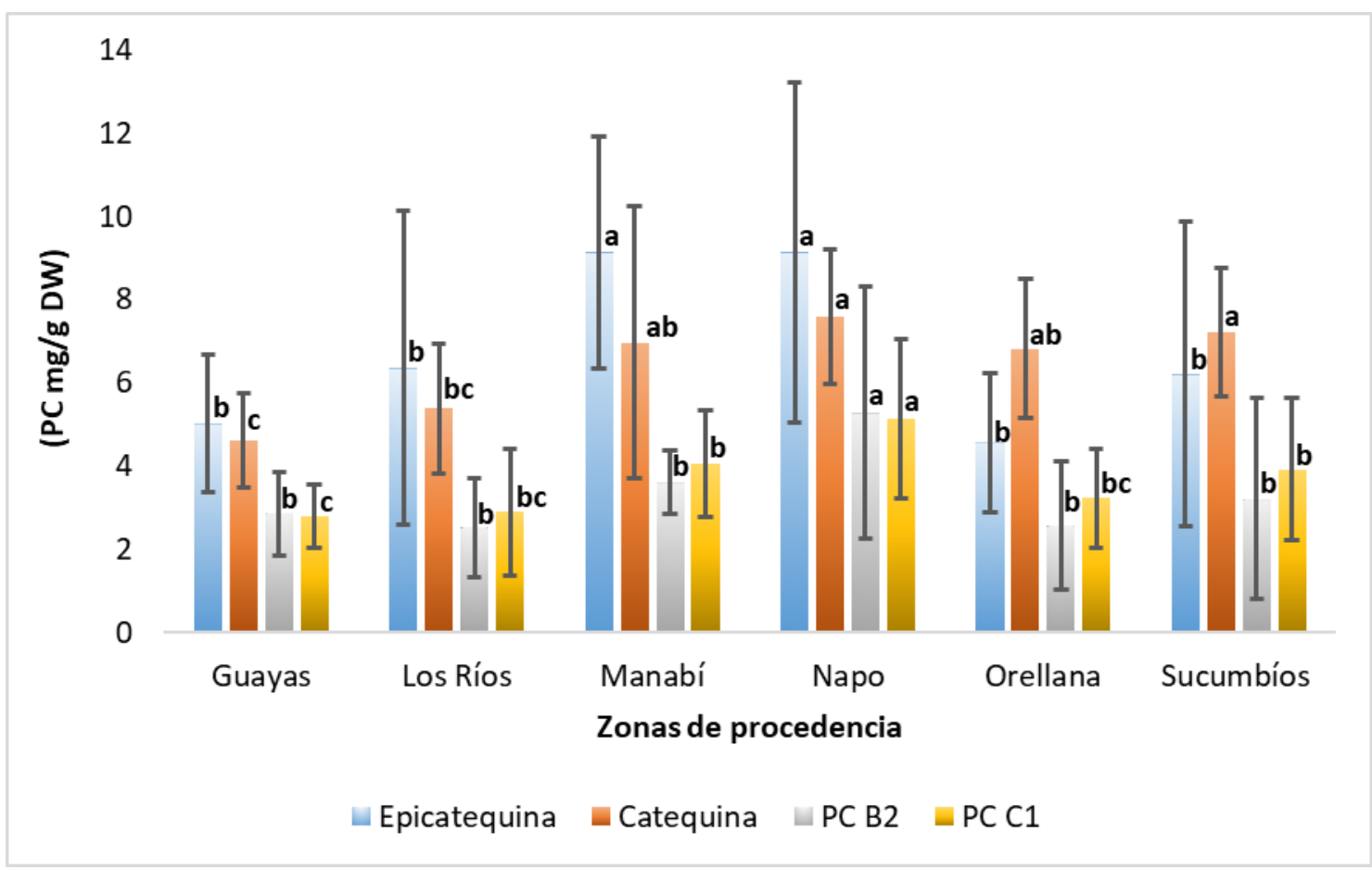

Figura 16. Contenido promedio por provincia de (-)-epicatequina, (+)-catequina, procianidina (PC) B2 y C1 en cacao Nacional ecuatoriano. Resultados expresados como la media $\pm S D(n=15)$. Diferentes letras indican diferencias estadísticamente significativas $(P<0.05)$ entre provincias.

El análisis de varianza (ANOVA) de los resultados, permitió establecer que existe diferencias estadísticas significativas $(p<0,05)$, para el contenido de $(-)$-epicatequina, (+)-catequina y procianidina B2 y C1 entre provincias y cantones, estableciéndose que existe un efecto de la zona de producción sobre el contenido de estos compuestos fitoquímicos. De igual manera, los datos obtenidos demostraron que el cacao 
"Nacional" procedente de las provincias de Manabí (región Costa del Pacífico) y Napo (región Amazónica), presentan el mayor contenido de flavan-3-oles y procianidinas con relación a las demás provincias en estudio.

La identificación y cuantificación de los compuestos fenólicos y su relación con la capacidad antioxidante, permitirá agregar valor al cacao "Nacional" ecuatoriano por sus propiedades funcionales. Esto se debe a que, en el mercado mundial durante las últimas décadas, se ha generado una mayor concientización sobre el consumo de alimentos saludables, lo cual ha llevado a los consumidores a cambiar sus hábitos alimenticios, adquiriendo cada vez más productos libres de azúcar, bajos en grasa y calorías (PROECUADOR, 2013).

Este comportamiento se ha observado con gran fuerza en el consumo de chocolates, puesto que; este producto ha sido tradicionalmente reconocido como una golosina. Sin embargo, los productores y demás actores en la industria chocolatera, están enfocándose en demostrar los beneficios en la salud que tienen los chocolates, en especial aquellos con el mayor porcentaje de solidos de cacao, conocidos como chocolate negro (contenido de solidos de cacao > 35\%) (Figura 17).

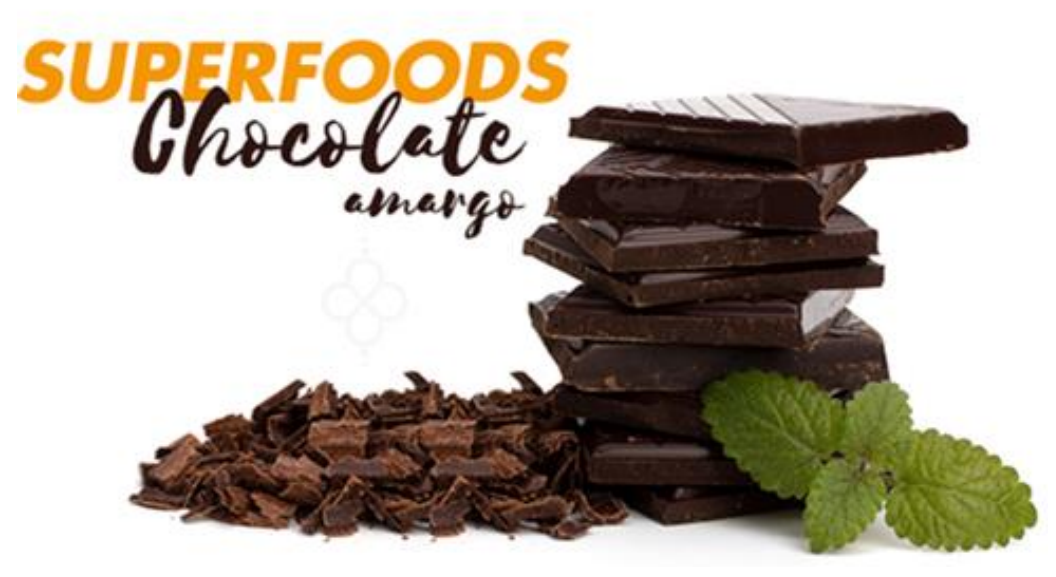

Figura 17. Chocolate negro rico en antioxidantes

\subsection{Contenido de grasa y perfil de ácidos grasos en cacao "Nacional" ecuatoriano. (Resultados relevantes de la Publicación 2).}

La grasa o manteca de cacao es el componente más abundante de los granos de cacao (48 - 57\% aproximadamente), se la considera como su principal producto de 
transformación. En la industria chocolatera, la grasa de cacao es la responsable de la rápida fusión del chocolate en la boca y le concede un brillo especial. Además, influye directamente en su tiempo de vida útil. (CAOBISCO/ECA/CFC, 2015; D'Souza et al., 2017; do Carmo et al., 2017; Jahurul et al., 2013; Loureiro et al., 2017; Naik \& Kumar, 2014; Servent et al., 2018). Por lo cual, la caracterización del contenido total y la composición bioquímica de la grasa de cacao, constituye un parámetro muy importante de calidad, que influye en su precio en el mercado internacional. En este sentido, debido a la escasa información de este componente importante en almendras de cacao "Nacional" ecuatoriano, en la presente tesis doctoral se evalúo el contenido total de grasa y su composición de ácidos grasos.

\subsubsection{Contenido de grasa total.}

El contenido de grasa en el cacao es un parámetro de calidad muy importante, puesto que su rendimiento en el grano, influye en el precio pagado en el mercado por el cacao de diferentes orígenes. Estudios previos realizados por diferentes autores, han demostrado que el contenido de TF varía en función de factores como: el origen geográfico y genético de los granos, así como; por factores ambientales (Loureiro et al., 2017; Riaño et al., 2016).

El cacao "Nacional" ecuatoriano procedente de la región Litoral (Costa del Pacífico), presentó un contenido promedio de grasa total por provincia, en el rango de $46.07 \pm$ 0.40 a 47,63 $\pm 1.08 \mathrm{~g}$ de grasa/100 g DW; mientras que, en la región Amazónica los contenidos promedio variaron en el rango de $50.56 \pm 0.23$ a $51.66 \pm 0.45 \mathrm{~g}$ de grasa/100 g DW (Figura 18). Estos resultados, concuerdan con estudios previos realizados por diferentes autores (Loureiro et al., 2017; Naik \& Kumar, 2014), los mismos que indican que el cacao Fino de origen Trinitario y Criollo presenta contenidos inferiores de grasa $(<52 \%)$, mientras que; el cacao ordinario de origen Forastero presenta mayores contenidos de grasa $(>52 \%)$.

El análisis estadístico de los resultados, demostró que existe un efecto significativo de la zona geográfica de producción $(\mathrm{p}<0.05)$, sobre el contenido de grasa total de las almendras de cacao, demostrándose que; el cacao de la región Amazónica presenta mayores contenidos de grasa, con relación a los procedentes de la región Litoral (Costa del Pacífico). Esto constituye un indicador de calidad que permite agregar valor al cacao de la Amazonía ecuatoriana, destacándose su mayor rendimiento en la 
producción de chocolate, mismo que, depende del contenido de grasa de las almendras.

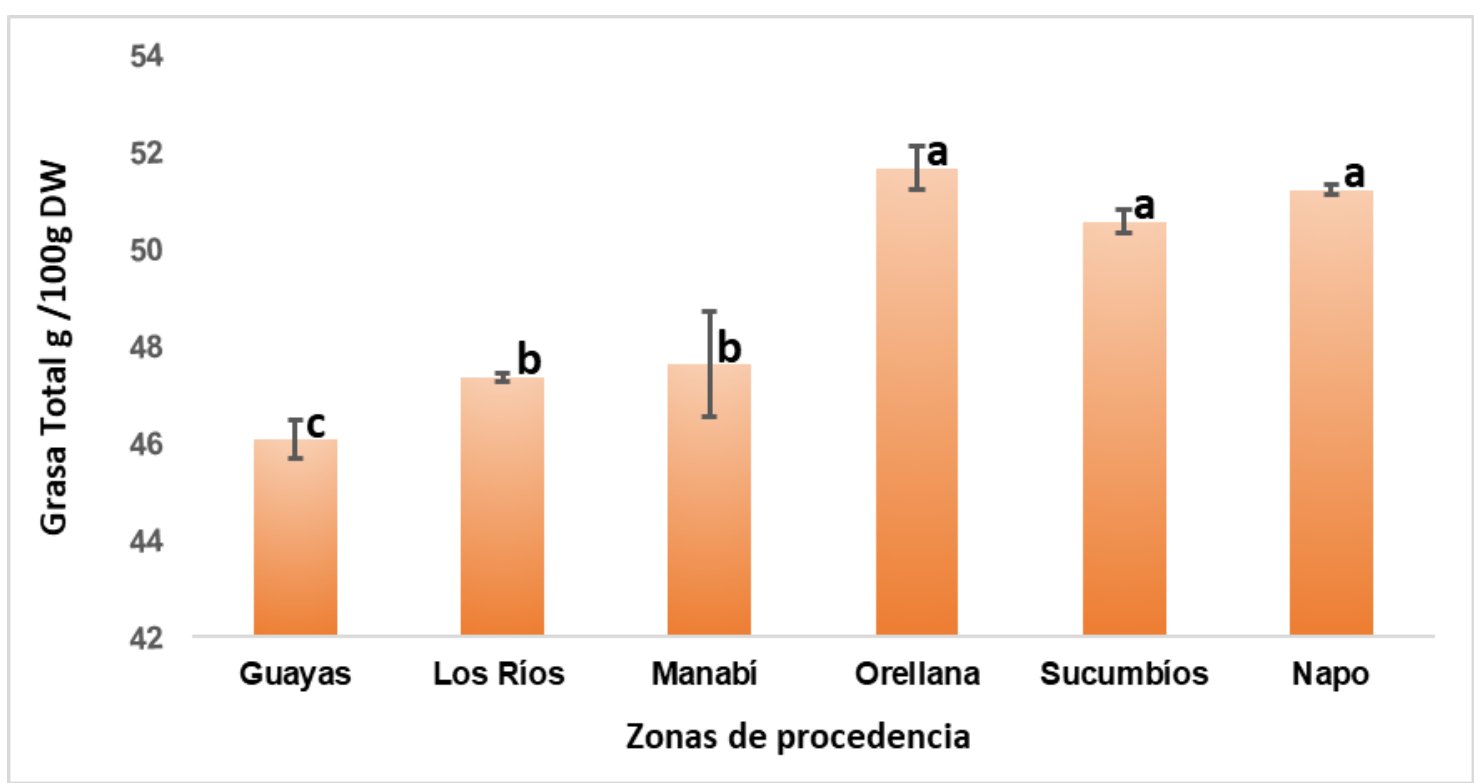

Figura 18. Contenido promedio por provincia de grasa total en cacao Nacional ecuatoriano. Resultados expresados como la media \pm SD $(n=15)$. Diferentes letras indican diferencias estadísticamente significativas $(P<0.05)$ entre provincias.

\subsubsection{Contenido de ácidos grasos}

El perfil de ácidos grasos en el cacao, es un parámetro de calidad muy estudiado por diferentes laboratorios de la industria chocolatera, puesto que su presencia y disposición estructural, determina las propiedades especiales de la grasa de cacao con relación a otras grasas vegetales. La composición de ácidos grasos en la grasa de cacao, establece el grado de cristalización del chocolate y sus propiedades de fusión en la boca (Jahurul et al., 2013; Naik \& Kumar, 2014; Riaño et al., 2016).

En este contexto, dentro de la investigación realizada en cacao "Nacional" ecuatoriano, se determinó el perfil de ácidos grasos en la fracción lipídica de los granos, así como; la variación del contenido de estos ácidos grasos en función de la zona geográfica de producción. Los resultados obtenidos, permitieron establecer que el perfil de FA de esta variedad de cacao está constituido por 14 FA, de los cuales cuatro FA (ácido palmítico $\mathrm{C} 16: 0$, ácido esteárico $\mathrm{C18:0}$, ácido oleico $\mathrm{C18:1}$, ácido linoleico C18:2), constituyen el $97.70 \%$ del total de FA (Figura 19). 


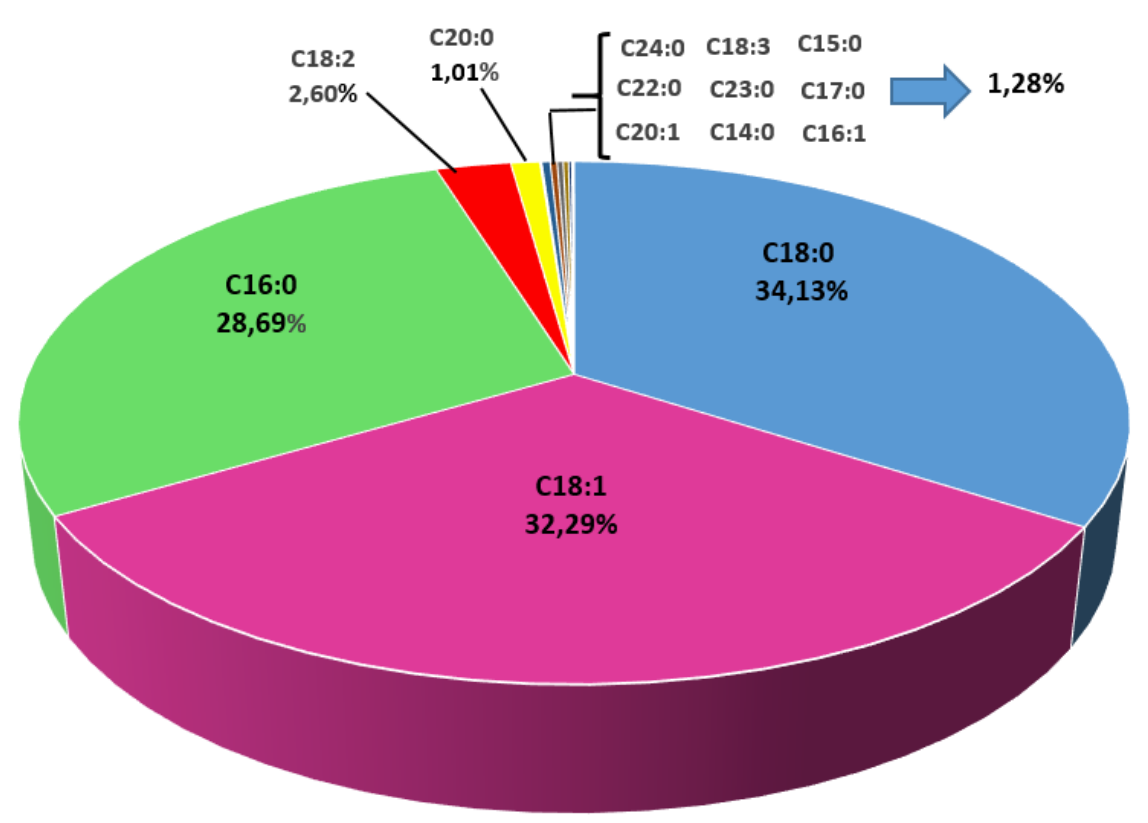

Figura 19. Composición porcentual de ácido grasos en cacao Nacional ecuatoriano. C14:0 (ácido mirístico), C15:0 (ácido pentadecanoico), C16:0 (ácido palmítico), C16:1 (ácido palmitoleico), C17:0 (ácido heptadecanoico), C18:0 (ácido esteárico), C18:1 (ácido oleico), C18:2 (ácido linoleico), C20:0 (ácido araquídico), C20:1 (ácido eicosenoico); C18:3 (ácido linolenico), C22:0 (ácido behénico), C23:0 (ácido tricosenoico), C24:0 (ácido lignocérico).

Los contenidos promedio por provincia de los principales FA del cacao ecuatoriano, varían en el rango de $27.65 \pm 1.49$ a $29.08 \pm 0.72 \mathrm{~g} / 100 \mathrm{~g}$ CF para ácido palmítico C16:0, de $33.59 \pm 1.03$ a $35.23 \pm 1.08 \mathrm{~g} / 100 \mathrm{~g}$ CF para ácido esteárico C18:0, de 31.92 \pm 0.80 a $32.95 \pm 1.03 \mathrm{~g} / 100 \mathrm{~g}$ CF para ácido oleico C18:1 y de $2.29 \pm 0.49$ a $2.99 \pm$ $0.41 \mathrm{~g} / 100 \mathrm{~g}$ CF para ácido linoleico C18:2. Adicionalmente, se estableció que el contenido de estos FA varía en función de la zona geográfica de producción $(p<0.05)$, por lo cual; el perfil de FA de la grasa de cacao, constituye un parámetro que puede contribuir a la certificación de origen de los granos de cacao, además; se presenta como un factor determinante de la calidad de las almendras para la fabricación de chocolate.

Desde el punto de vista nutricional, el cacao Nacional ecuatoriano presenta contenidos más altos de ácidos grasos saturados (SFA), con relación a los ácidos grasos insaturados (UFA); la relación promedio entre estos dos grupos (S/U) por provincia varía en el rango de 1,79 $\pm 0,05$ a $1.84 \pm 0.02$. Demostrándose, que esta relación varía significativamente $(p<0.05)$, en función del origen geográfico del cacao. 
La presencia mayoritaria de ácidos grasos saturados sobre los insaturados en el perfil de ácidos grasos de la grasa de cacao, es considerado como negativo desde el punto de vista nutricional, Sin embargo; estudios realizados previamente, han demostrado que el ácido esteárico (C18:0), es un ácido graso neutral que no tiene efectos aterogénicos o colesterogénicos, puesto que; es desaturado rápidamente en el cuerpo humano y se transforma en ácido oleico (C18:1) (Riaño et al., 2016).

Por todo lo anterior se puede establecer que, el cacao es un alimento rico en compuestos bioactivos, de gran interés para la salud, pero con una composición diferente según la zona de producción dentro de uno de los países mayores productores de cacao, ofreciendo cacaos de diferente calidad. Debido a ello, el disponer de unas buenas herramientas de caracterización permitirá establecer la calidad del cacao con métodos objetivos. Estos métodos permitirán una caracerización nutricional, pero también geográfica, lo cual puede evitar fraudes con la demonimación de origen de este alimento. 


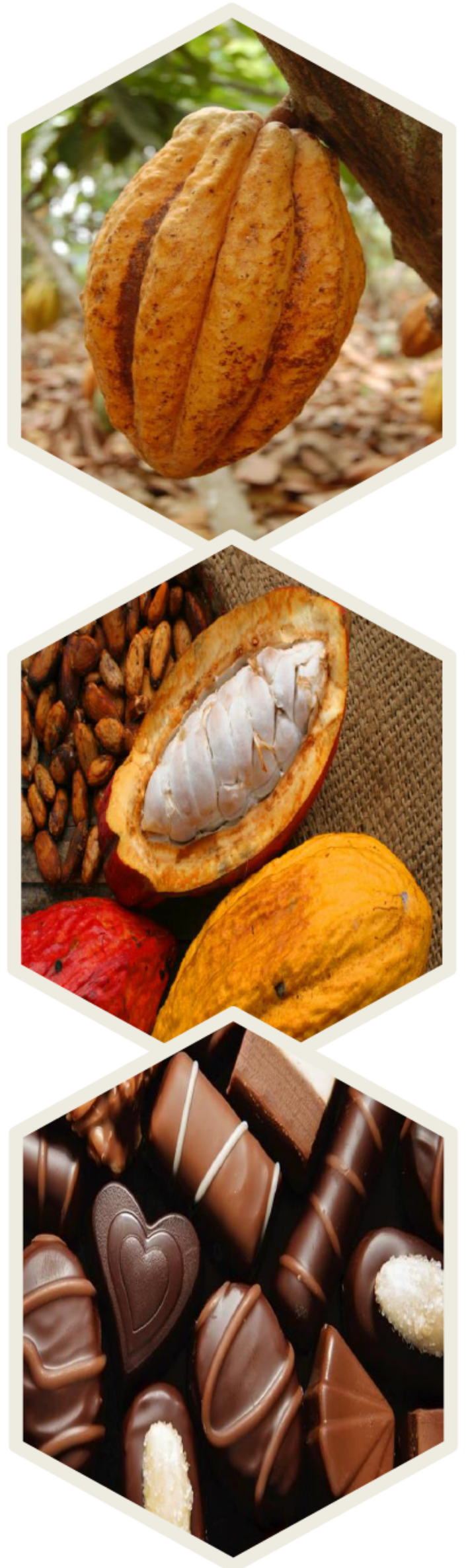

\section{Capítulo V}

Conclusiones 
1. El cacao Nacional ecuatoriano, presenta altos contenidos de teobromina y menores contenidos de cafeína, los cuales varían en función de la zona geográfica (mayor concentración en la región Amazónica). Esta característica influye en la calidad sensorial de los granos, puesto que las metilxantinas están relacionadas con el sabor amargo de las almendras de cacao.

2. El cacao Nacional ecuatoriano presenta valores de la relación TBR/CAF en un rango que lo clasifican como cacao tipo Trinitario. Este resultado confirma el origen genético del cacao ecuatoriano, puesto que actualmente la mayor parte de materiales sembrados en este país, son híbridos de este tipo especial de cacao con materiales Trinitarios introducidos de Venezuela y Trinidad. Adicionalmente, este resultado demuestra que el cacao ecuatoriano es Fino, puesto que comercialmente se reconoce a los cacaos de tipo Trinitario y Criollo como materiales para elaboración de chocolates Finos.

3. La alta concentración en polifenoles del cacao Nacional "Fino de Aroma", le otorga un valor nutricional añadido para poder ser comercializado, además de su sabor y aroma especial, por sus propiedades funcionales, permitiendo abrir nuevos nichos de mercado relacionados con efectos beneficiosos para la salud.

4. La fracción lipídica es el componente más abundante del cacao Nacional ecuatoriano (46.98 al $51.22 \%$ del total de fitoquímicos), el mismo que varía en función de la zona geográfica de producción (mayores contenidos en la región Amazónica). Esta particularidad, determina un mayor precio en el mercado internacional, puesto que se ha demostrado que el cacao de estas zonas es Fino y con mayores contenidos de grasa.

5. Los componentes bioquímicos determinados en el cacao Nacional ecuatoriano, constituyen una herramienta objetiva para evaluar la calidad de los granos de cacao para exportación. Puesto que, pueden servir para definir su origen genético, el grado de fermentación, las potenciales propiedades salutísticas, el contenido y la calidad de grasa, teniendo en cuenta el efecto de la zona geográfica de producción sobre el contenido de estos fitoquímicos, la presente información permite a los productores e industriales ecuatorianos identificar zonas de producción con diferentes características de calidad. Esto ayudará en corto plazo a certificar su origen y calidad especial, con miras a obtener mejores precios en el mercado internacional. 


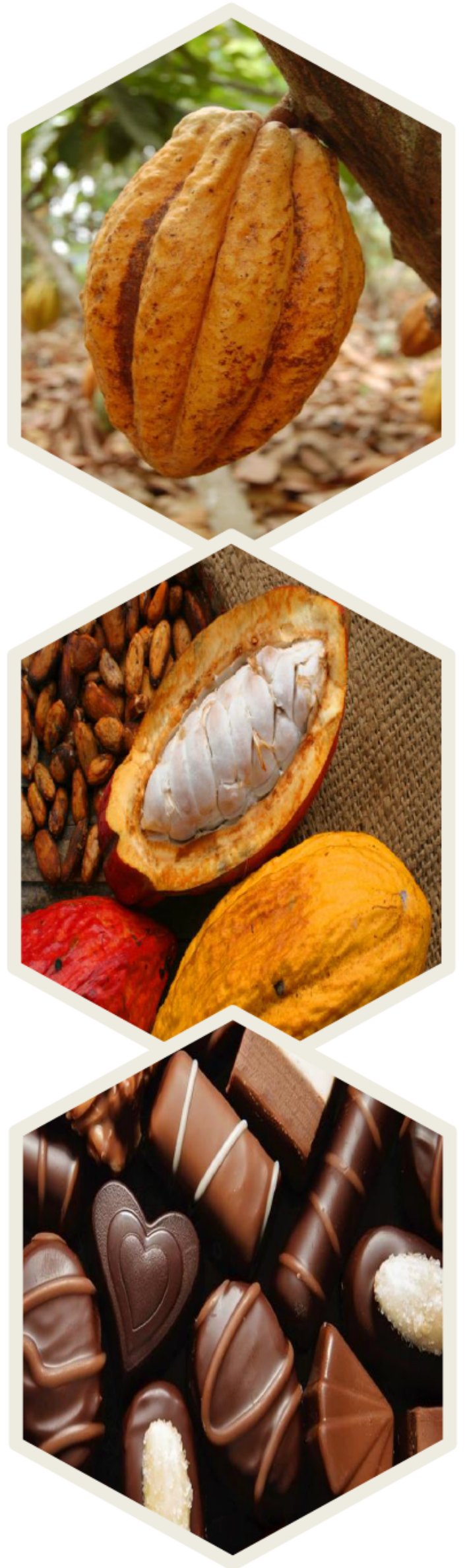

\section{Capítulo VII} Referencias

\section{Bibliográficas}


Acierno, V., Yener, S., Alewijn, M., Biasioli, F., \& van Ruth, S. (2016). Factors contributing to the variation in the volatile composition of chocolate: Botanical, and geographical origin of the cocoa beans, and brand-related formulation and processing. Food Research International. https://doi.org/10.1016/j.foodres.2016.03.022

Adeyeye, E., \& Akinyeye, R. (2017). Effect of farm and industrial processing on the amino acid profile of cocoa beans. https://doi.org/10.1016/j.foodchem.2009.04.127

Afoakwa, E. O., Paterson, A., Fowler, M., \& Ryan, A. (2008). Flavor formation and character in cocoa and chocolate: A critical review. https://doi.org/10.1080/10408390701719272

Afoakwa, E. O., Quao, J., Simpson Budu, A., Takrama, J., \& Kwesi Saalia, F. (2011). Effect of pulp preconditioning on acidification, proteolysis, sugars and free fatty acids concentration during fermentation of cocoa (Theobroma cacao) beans. https://doi.org/10.3109/09637486.2011.581224

Amores., F., Butler., D. ., Ramos., G., Sukha., D. A., Espín, S., Gomez., A., Seguine., E. (2007). Project to establish the physical, chemical and organoleptic parameters to differentiate between fine or flavor and bulk cocoa. Quevedo, Ecuador.

Aprotosoaie, A. C., Luca, S. V., \& Miron, A. (2016). Flavor Chemistry of Cocoa and Cocoa Products-An Overview. Comprehensive Reviews in Food Science and Food Safety, 15(1), 73-91. https://doi.org/10.1111/1541-4337.12180

Aprotosoaie, A. C., Luca, S. V., Miron, A., Arvelo Sánchez, M. A., González León, D., Maroto Arce, S., ... Batista, L. (2017). Flavor Chemistry of Cocoa and Cocoa Products-An Overview. In Santo Domingo, Republica Dominicana.Centro para el Desarrollo Agropecuario y Forestal CEDAF (Vol. 15). https://doi.org/10.1016/S0365-6691(10)70034-4

Araujo, Q., Fernandes, C., Ribeiro, D., Efraim, P., Steinmacher, D., Lieberei, R., Araujo, T. (2014). Cocoa Quality Index - a Proposal Quintino. Food Control. https://doi.org/10.1016/j.foodcont.2014.05.003

Asociación Nacional de Exportadores de Cacao. (2015). Historia del cacao. Retrieved June 3, 2020, from ANECACAO website: http://www.anecacao.com/es/quienessomos/historia-del-cacao.html

Balladares Grazzo, C. (2015). Caracterización físico - química de los lixiviados del cacao y café del litoral ecuatoriano, como potenciales fuentes de producción de bioetanol. UNIVERSIDAD DE LAS PALMAS DE GRAN CANARIA. 
Batista, L. (2009). Guía Técnica El Cultivo de Cacao. In Santo Domingo, Republica Dominicana.Centro para el Desarrollo Agropecuario y Forestal CEDAF (Vol. 2). https://doi.org/10.1016/S0365-6691(10)70034-4

Belščak, A., Komes, D., Horžić, D., Ganić, K., \& Karlović, D. (2009). Comparative study of commercially available cocoa products in terms of their bioactive composition. Food Research International, 42, 707-716. https://doi.org/10.1016/j.foodres.2009.02.018

Brunetto, M. del R., Gutiérrez, L., Delgado, Y., Gallignani, M., Zambrano, A., Gómez, Á., Romero, C. (2007). Determination of theobromine , theophylline and caffeine in cocoa samples by a high- performance liquid ... Determination of theobromine , theophylline and caffeine in method with on-line sample cleanup in a switchingcolumn system. Food Chemistry, 100(June), 459-467. https://doi.org/10.1016/j.foodchem.2005.10.007

Cabrera-Soto, L., Salinas-Moreno, Y., Velázquez-Cardelas, G., \& Espinosa-Trujillo, E. (2009). Contenido de fenoles solubles e insolubles en las estructuras del grano de maíz y su relación con propiedades físicas. Agrociencia, 43(8), 827-839.

Caligiani, A., Marseglia, A., Prandi, B., \& Palla, G. (2016). Pattern. Food Chemistry. https://doi.org/10.1016/j.foodchem.2016.05.072

Caligiani, A., Palla, L., Acquotti, D., Marseglia, A., \& Palla, G. (2014). Application of 1H NMR for the characterisation of cocoa beans of different geographical origins and fermentation levels. Food Chemistry, 157, 94-99. https://doi.org/10.1016/j.foodchem.2014.01.116

Cambrai, A., Marchioni, E., Julien-david, D., \& Marcic, C. (2017). Discrimination of Cocoa Bean Origin by Chocolate Polyphenol Chromatographic Analysis and Chemometrics. Food Analysis Methods, 10, 1991-2000. https://doi.org/10.1007/s12161-016-0744-7

Campos-Vega, R., Nieto-Figueroa, K. H., \& Oomah, B. D. (2018). Cocoa (Theobroma cacao L.) pod husk: Renewable source of bioactive compounds. Trends in Food $\begin{array}{llll}\text { Science } \quad \text { and } & \text { 172-184. }\end{array}$ https://doi.org/10.1016/j.tifs.2018.09.022

CAOBISCO, ECA, \& FCC. (2015). Cocoa Beans: Chocolate and Cocoa Industry Quality Requirements.

Carrasco, O. (2015). Obtención de harina baja en gluten a partir de la cascarilla de cacao de las variedades CCN-51 y Nacional. UNIVERSIDAD TÉCNICA DE 


\section{MACHALA.}

Carrillo, L. C., Londoño-Londoño, J., \& Gil, A. (2014). Comparison of polyphenol, methylxanthines and antioxidant activity in Theobroma cacao beans from different cocoa-growing areas in Colombia. Food Research International, 60, 273-280. https://doi.org/10.1016/j.foodres.2013.06.019

Castro, M. C., Villagarcía, H., Nazar, A., Arbeláez, L. G., Massa, M. L., Del Zotto, H., Francini, F. (2020). Cacao extract enriched in polyphenols prevents endocrinemetabolic disturbances in a rat model of prediabetes triggered by a sucrose rich diet. Journal of Ethnopharmacology, 247(June 2019), 112263. https://doi.org/10.1016/j.jep.2019.112263

Colizzi, C. (2019). The protective effects of polyphenols on Alzheimer's disease: A systematic review. Alzheimer's and Dementia: Translational Research and Clinical Interventions, 5, 184-196. https://doi.org/10.1016/j.trci.2018.09.002

Criado, M. N., Morelló, J. R., Motilva, M. J., \& Romero, M. P. (2004). Effect of Growing Area on Pigment and Phenolic Fractions of Virgin Olive Oils of the Arbequina Variety in Spain. Journal of the American Oil Chemists' Society, 81(7), 633-640. https://doi.org/10.1007/s11746-004-954-z

Cros, E., \& Vincent, J. (1982). Recherche d'un indice de fermentation du cacao, 1: Evolution des tanins et des phénols totaux de la feve. Café, Cacao, Thé (Francia), 26(2), 109-114.

D’Souza, R., Grimbs, S., Behrends, B., Bernaert, H., Ullrich, M. S., \& Kuhnert, N. (2017). Origin-based polyphenolic fi ngerprinting of Theobroma cacao in unfermented and fermented beans. Food Research International, 99(March), 550559. https://doi.org/10.1016/j.foodres.2017.06.007

Dand, R. (2011a). Agronomics of international cocoa production. In The International Cocoa Trade (pp. 23-64). https://doi.org/10.1016/B978-0-85709-125-3.50002-1

Dand, R. (2011b). History and origins of the international cocoa trade. In The International Cocoa Trade (Third edit, pp. 1-22). https://doi.org/10.1016/B978-085709-125-3.50001-X

Davrieux, F., Assemat, S., Boulanger, R., \& Cros, E. (2003). Determination of cocoa purine content by near infrared spectroscopy [Poster]. In: 14th International Cocoa Research Conference, 13-18 October 2003, Accra, Ghana. - Montpellier: Cirad, 2003, 1 p.

de Souza, P. A., Moreira, L. F., Sarmento, D. H. A., \& da Costa, F. B. (2018). Cacao- 
Theobroma cacao. Exotic Fruits, 3(2001), 69-76. https://doi.org/10.1016/b978-012-803138-4.00010-1

De Taeye, C., Bodart, M., Caullet, G., \& Collin, S. (2017). Roasting conditions for preserving cocoa flavan-3-ol monomers and oligomers: interesting behaviour of Criollo clones. Journal of the Science of Food and Agriculture, 97(12), 4001-4008. https://doi.org/10.1002/jsfa.8265

Diab, J., Hertz-Schünemann, Romy Streibel, T., \& Zimmer-Mann, R. (2014). On-line measurement of volatile organic compounds released during roasting ofcocoa beans. Food Research International, 63, 344-352. https://doi.org/10.1016/j.foodres.2014.04.047

Diomande, D., Antheaume, I., Leroux, M., Lalande, J., Balayssac, S., Remaud, G. S., \& Tea, I. (2015). Multi-element, multi-compound isotope profiling as a means to distinguish the geographical and varietal origin of fermented cocoa ( Theobroma cacao $\mathrm{L}$.) beans. Food Chemistry, 188, 576-582. https://doi.org/10.1016/j.foodchem.2015.05.040

do Carmo, B., Campos, R., da Silva, R., Abreu, M. B., \& Lopes, A. (2017). Bioactive amines and phenolic compounds in cocoa beans are affected by fermentation. Food Chemistry, 228, 484-490. https://doi.org/10.1016/j.foodchem.2017.02.004

EL Telégrafo. (2019). Exportaciones de cacao subieron 4,65\% en 2018. Diario El Telégrafo.

Enríquez, G. (1985). Curso sobre el cultivo del cacao (Centro Agr). Retrieved from https://books.google.es/books?hl=es\&lr=\&id=eZgOAQAAIAAJ\&oi=fnd\&pg=PA5\&d $\mathrm{q}=$ enríquez+1985+cacao\&ots=lqxK0-Vm7N\&sig=0V-Psdo9kFZ6heOCCugnlTh3lc\#v=onepage \&q=enríquez 1985 cacao\&f=false

Espín, S., \& Samaniego, I. (2016). Manual para análisis de parámetros químicos asociados a la calidad del cacao. In Manual Nro 105. Retrieved from http://181.112.143.123/bitstream/41000/2827/1/iniapsc322est.pdf

Espín, S., Samaniego, I., Wakao, H., \& Juan, J. (2007). La relación teobromina/cafeina asociada a la calidad del cacao ecuatoriano. Alimentos Ciencia e Ingeniería, 16(2), 107-109.

Exporters-Ecuador National Association of Cocoa. (2016). Sabor Arriba. Guayaquil, Ecuador.

Fernández, V., Yee, A., Sulbarán, B., \& Peña, J. (2014). Actividad antioxidante y contenido de polifenoles en chocolates comerciales venezolanos. Revista de La 
Facultad de Agronomia, 31(1), 129-144.

Franco-Agurto, G., \& Suárez-Quirumbay, K. (2014). Determinación del contenido de polifenoles y actividad antioxidante de una bebida láctea elaborada a base de residuos agroindustriales de cacao, café y naranja. Escuela Superior Politécnica del Litoral.

García-Vidal, G., Guzmán-Vilar, L., \& Campdesuñer, R. P. (2017). Research Trends in Cocoa : Opportunities for Research in Santo. SATHIRI, Sembrador, 12(2), 22.

Gibson, M., \& Newsham, P. (2018). Chocolate/Cacao. Food Science and the Culinary Arts, 341-352. https://doi.org/10.1016/b978-0-12-811816-0.00017-8

Gómez-Juaristi, M., González-Torres, L., Bravo, L., Vaquero, M. P., Bastida, S., \& Sánchez-Muniz, F. J. (2011). Efectos beneficiosos del chocolate en la salud cardiovascular. Nutricion Hospitalaria, 26(2), 289-292. https://doi.org/10.3305/nh.2011.26.2.5016

Grimbs, S., \& Ullrich, M. S. (2017). Origin-based polyphenolic fingerprinting of Theobroma cacao in unfermented and fermented beans. Food Research International, (June), 0-1. https://doi.org/10.1016/j.foodres.2017.06.007

Guanga, S. (2018). Estudio y aprovechamiento de los residuos del cacao de la compañía Nestlé como estrategia comercial. Universidad De Guayaquil.

Gutiérrez Maydata, B. A. (2002). Chocolate, polifenoles y protección a la salud. Acta Farmaceutica Bonaerense, 21(2), 149-152.

Hartel, R. W., von Elbe, J. H., \& Hofberger, R. (2018). Confectionery Science and Technology. In Confectionery Science and Technology. https://doi.org/10.1007/978-3-319-61742-8

Hooper, L., Kay, C., Abdelhamid, A., Kroon, P. A., Cohn, J. S., Rimm, E. B., \& Cassidy, A. (2012). Effects of chocolate, cocoa, and flavan-3-ols on cardiovascular health: a systematic review and meta-analysis of randomized trials $1-3$. American Journal of Clinical Nutrition, 95, 740-751. https://doi.org/10.3945/ajcn.111.023457.INTRODUCTION

Hue, C., Brat, P., Gunata, Z., Samaniego, I., Servent, A., Morel, G., ... Davrieux, F. (2014). Near infra-red characterization of changes in flavan-3-ol derivatives in cocoa (Theobroma cacao L.) As a function of fermentation temperature. Journal of Agricultural and Food Chemistry, 62(41), 10136-10142. https://doi.org/10.1021/jf501070d 
Instituto Nacional Ecuatoriano de Normalización. Granos de cacao. Requisitos. , (2018).

International Cocoa Organization. (2017). Annual Report 2014/2015.

Jahurul, M. H. A., Zaidul, I. S. M., Norulaini, N. A. N., Sahena, F., Jinap, S., Azmir, J., ... Mohd Omar, A. K. (2013). Cocoa butter fats and possibilities of substitution in food products concerning cocoa varieties, alternative sources, extraction methods, composition, and characteristics. Journal of Food Engineering, 117(4), 467-476. https://doi.org/10.1016/j.jfoodeng.2012.09.024

Jimenez, J., Amores, F., \& Solórzano, E. (2014). Componentes de identidad para reconocer las diferencias del cacao que se produce en varias regiones del Ecuador. Quevedo, Ecuador.

Jimenez, J., Amores, F., \& Solórzano, E., Jiménez, J., Amores, F., \& Solórzano, E. (2014). Componentes de identidad para reconocer las diferencias del cacao que se produce en varias regiones del Ecuador. Estación Experimental Tropical Pichilingue. INIAP. Boletín Técnico: 164. In Estación Experimental Tropical Pichilingue INIAP. (Vol. 164). Quevedo, Ecuador, Ecuador.

Jiménez, J., Amores, F., Nicklin, C., Rodríguez, D., Zambrano, F., Bolaños, M., Cedeño, P. (2011). Micro fermentación y análisis sensorial para la selección de árboles superiores de cacao. Retrieved from file:///C:/Users/Owner/Downloads/Boletín técnico Nº 140 (2).PDF

Jovellanos-Fernández, E. (2016). Estudio del contenido de compuestos bioactivos del cacao y su aplicación en la obtención de un ingrediente rico en polifenoles para el diseño de un chocolate enriquecido (Universidad de Murcia). https://doi.org/10.17161/bi.v1i0.3

Jovellanos Fernandez, E. (2016). Estudio del contenido de compuestos bioactivos del cacao y su aplicación en la obtención de un ingrediente rico en (poli)fenoles para el diseño de un chocolate enriquecido (UNIVERSIDAD DE MURCIA). https://doi.org/10.17161/bi.v1i0.3

Kerimi, A., \& Williamson, G. (2015). The cardiovascular benefits of dark chocolate. Vascular Pharmacology, 71, 11-15. https://doi.org/10.1016/j.vph.2015.05.011

Kim, J., Lee, K. W., \& Lee, H. J. (2011). Cocoa (Theobroma cacao) Seeds and Phytochemicals in Human Health. In Nuts and Seeds in Health and Disease Prevention (pp. 351-360). https://doi.org/10.1016/B978-0-12-375688-6.10042-8

Konger Edem, J., Hinneh, M., Van de Walle, D., Afoakwa, E. O., Boeckx, P., \& 
Dewettinck, K. (2016). Factors influencing quality variation in cocoa (Theobroma cacao) bean flavour profile - A review. Food Research International, 82, 44-52. https://doi.org/10.1016/j.foodres.2016.01.012

Kongor, J. E., Hinneh, M., de Walle, D. Van, Afoakwa, E. O., Boeckx, P., \& Dewettinck, K. (2016). Factors influencing quality variation in cocoa ( Theobroma cacao ) bean flavour profile - A review. Food Research International, 82(January), 44-52. https://doi.org/10.1016/j.foodres.2016.01.012

Krähmer, A., Engel, A., Kadow, D., Ali, N., Umaharan, P., Kroh, L. W., \& Schulz, H. (2015). Fast and neat - Determination of biochemical quality parameters in cocoa using near infrared spectroscopy. 181, 152-159. https://doi.org/10.1016/j.foodchem.2015.02.084

Lefeber, T., Papalexandratou, Z., Gobert, W., Camu, N., \& Vuyst, L. De. (2012). Onfarm implementation of a starter culture for improved cocoa bean fermentation and its in $\mathrm{fl}$ uence on the $\mathrm{fl}$ avour of chocolates produced thereof. Food Microbiology, 30(2), 379-392. https://doi.org/10.1016/j.fm.2011.12.021

Lim, T. K. (2012). Edible Medicinal and Non Medicinal Plants Fruits. In Edible Medicinal and Non Medicinal Plants: Volume 3, Fruits (Vol. 3, pp. 1-159). https://doi.org/10.1007/978-94-007-2534-8

Lipp, M., \& Anklam, E. (1998). Review of cocoa butter and alternative fats for use in chocolate. Food Chemistry, 62(1), 73-97. https://doi.org/10.1016/S03088146(97)00160-X

Loor, R. G., Risterucci, A. M., Courtois, B., Fouet, O., Jeanneau, M., Rosenquist, E., Lanaud, C. (2009). Tracing the native ancestors of the modern Theobroma cacao L. population in Ecuador. Tree Genetics and Genomes, 5(3), 421-433. https://doi.org/10.1007/s11295-008-0196-3

López-Guerrero, A. (2017). Producción y comercialización de cacao Fino de Aroma en el Ecuador - Año 2012-2014". In Superintendencia de Control del Poder del Mercado (Vol. 102). https://doi.org/10.1002/ejsp.2570

Loureiro, G. A. H. A., Araujo, Q. R., Sodré, G. A., Valle, R. R., Souza, J. O., Ramos, E. M. L. S., ... Grierson, P. F. (2017). Cacao quality: Highlighting selected attributes. Food Reviews International, 33(4), 382-405. https://doi.org/10.1080/87559129.2016.1175011

Ludovici, V., Barthelmes, J., Nägele, M., Enseleit, F., Ferri, C., Flammer, A., ... Sudano, I. (2017). Cocoa, Blood Pressure, and vascular Function. 4(August), 1- 
12. https://doi.org/10.3389/fnut.2017.00036

Marseglia, A., Acquotti, D., Consonni, R., Cagliani, L. R., Palla, G., \& Caligiani, A. (2016). HR MAS $1 \mathrm{H} \mathrm{NMR}$ and chemometrics as useful tool to assess the geographical origin of cocoa beans - Comparison with HR $1 \mathrm{H}$ NMR. Food Research International Journal, 85, 273-281. https://doi.org/10.1016/j.foodres.2016.05.001

Martín, M. A., \& Ramos, S. (2016). Cocoa polyphenols in oxidative stress: Potential health implications. Journal of Functional Foods, 27, 570-588. https://doi.org/10.1016/j.jff.2016.10.008

Martínez, R., Torres, P., Meneses, M. A., Figueroa, J. G., Pérez-Álvarez, J. A., \& Viuda-Martos, M. (2012). Chemical, technological and in vitro antioxidant properties of cocoa (Theobroma cacao L.) co-products. Food Research International, 49(1), 39-45. https://doi.org/10.1016/j.foodres.2012.08.005

Mata Anchundia, D., Suatunces Cuñumai, J., Munoz Macías, S., Bayas, A., \& Herrera Soler, M. (2018). Dimensión climática en el comercio justo para el cacao fino de aroma (Theobroma cacao I.). Revista de Estudios Empresariales. Segunda Época, 2(2), 86-102. https://doi.org/10.17561/ree.v2018n2.5

Ministry of Agriculture and Livestock. (2016). Reportes Dinámicos ESPAC. In Superficie, Producción y Rendimiento (2002-2016) (Vol. 2017). Quito, Ecuador.

Mondino, C., \& Ferrato, J. (2006). El análisis sensorial, una herramienta para la evaluación de la calidad desde el consumidor. Agromensajes, 16-48.

Motamayor, J. C., Lachenaud, P., Wallace da Silva, J., Loor, R., Kuhn, D., Brown, S., \& Schnell, R. (2008). Geographic and genetic population differentiation of the Amazonian chocolate tree ( Theobroma cacao L ) Geographic and Genetic Population Differentiation of the Amazonian Chocolate Tree ( Theobroma cacao $\mathrm{L}$ ). Plos One, 3(10). https://doi.org/10.1371/journal.pone.0003311

Mundaca Vidarte, G. A. (2016). Análisis de la calidad del grano de cacao mediante imágenes hiperespectrales usando técnicas de visión artificial. UNVERSIDAD DE PIURA.

Muñoz Hernández, G. C. (2018). Evaluación de la capacidad antioxidante en cacao Nacional fino de aroma (Theobroma cacao L.), de las principales zonas productoras del Ecuador. UNIVERSIDAD CENTRAL DEL ECUADOR.

Naik, B., \& Kumar, V. (2014). Cocoa Butter and Its Alternatives: A Reveiw. Journal of Bioresource Engineering and Technology, 1(March 2014), 7-17. 
Nara Batista, N., Pereira de Andrade, D., Lacerda Ramos, C., Ribeiro Dias, D., \& Freitas Schwan, R. (2016). Antioxidant capacity of cocoa beans and chocolate assessed by FTIR. Food Research International Journal, 1-7. https://doi.org/10.1016/j.foodres.2016.10.028

Ooi, T. S., Ting, A. S. Y., \& Siow, L. F. (2020). Influence of selected native yeast starter cultures on the antioxidant activities, fermentation index and total soluble solids of Malaysia cocoa beans: A simulation study. Lwt, 122(July 2019), 108977. https://doi.org/10.1016/j.lwt.2019.108977

Ooi, T. S., Ting, A. S. Y., Siow, L. F., Romanens, E., Freimüller Leischtfeld, S., Volland, A., ... Tavares Bisneto, J. D. (2020). Screening of lactic acid bacteria and yeast strains to select adapted anti-fungal co-cultures for cocoa bean fermentation. Food Chemistry, 290(October 2019), 7-12. https://doi.org/10.1016/j.Iwt.2019.108977

Oracz, J., \& Nebesny, E. (2014). Influence of roasting conditions on the biogenic amine content in cocoa beans of different Theobroma cacao cultivars. Food Research International, 55, 1-10. https://doi.org/10.1016/j.foodres.2013.10.032

Oracz, J., Nebesny, E., \& Dorota, Ż. (2019). Identification and quantification of free and bound phenolic compounds contained in the high-molecular weight melanoidin fractions derived from two di ff erent types of cocoa beans by UHPLC-DAD-ESIHR-MS. Food Research International, 115(August 2018), 135-149. https://doi.org/10.1016/j.foodres.2018.08.028

Othman, A., Ismail, A., Abdul, N., \& Adenan, I. (2007). Food Chemistry Antioxidant capacity and phenolic content of cocoa beans. Food Chemistry, 100, 1523-1530. https://doi.org/10.1016/j.foodchem.2005.12.021

Owusu, M., Petersen, M. A., \& Heimdal, H. (2011). Effect of fermentation method, roasting and conching conditions on the aroma volatiles of dark chocolate. Journal of Food Processing and Preservation, 1-11. https://doi.org/10.1111/j.17454549.2011.00602.x

Páramo, D., García-Alamilla, P., Salgado-Cervantes, M. A., Robles-Olvera, V. J., Rodríguez-Jimenes, G. C., \& García-Alvarado, M. A. (2010). Mass transfer of water and volatile fatty acids in cocoa beans during drying. Journal of Food Engineering, 99(3), 276-283. https://doi.org/10.1016/j.jfoodeng.2010.02.028

Parra, O., Gallego, A. M., Urrea, A., Rojas, L. F., Correa, C., \& Atehortúa, L. (2017). Biochemical precursor effects on the fatty acid production in cell suspension 
cultures of Theobroma cacao L. Plant Physiology and Biochemistry, 111, 59-66. https://doi.org/10.1016/j.plaphy.2016.11.013

Patras, M. A., Milev, B. P., Vrancken, G., \& Kuhnert, N. (2014). Identification of novel cocoa fl avonoids from raw fermented cocoa beans by HPLC - MS n. Food Research International Journal, 63, 353-359. https://doi.org/10.1016/j.foodres.2014.05.031

Pedan, V., Weber, C., Do, T., Fischer, N., Reich, E., Rohn, S., \& Andrew, S. (2017). HPTLC fi ngerprint pro fi le analysis of cocoa proanthocyanidins depending on origin and genotype. Food Chemistry, (August). https://doi.org/10.1016/j.foodchem.2017.08.109

Pereira-Caro, G., Borges, G., Nagai, C., Jackson, M. C., Yokota, T., Crozier, A., \& Ashihara, H. (2013). Profiles of Phenolic Compounds and Purine Alkaloids during the Development of Seeds of Theobroma cacao cv. Trinitario. Journal of Agricultural and Food Chemistry, 61, 427-434. https://doi.org/10.1021/jf304397m

Petersen, M. A., Blennow, A., \& Nielsen, D. S. (2014). Impact of starter cultures and fermentation techniques on the volatile aroma and sensory profile of chocolate. https://doi.org/10.1016/j.foodres.2014.04.032

Pinto-Mosquera, N. (2011). Propuesta de mejora a los factores que afectan la competitividad de la cadena productiva del cacao en grano de la empresa "Aroma Amazónico CIA. LTDA." Universidad de Las Américas.

Pontillon, J. (1997). Cacao et chocolat: Production, utilisation, caractéristiques. Paris: Tec et doc-Lavoisier.

Predan, G. M. I., Lazăr, D. A., \& Lungu, I. I. (2019). Cocoa industry-from plant cultivation to cocoa drinks production. Caffeinated and Cocoa Based Beverages: Volume 8. The Science of Beverages, 489-507. https://doi.org/10.1016/B978-012-815864-7.00015-5

PROECUADOR. (2013). Análisis del sector cacao y elaborados. Quito, Ecuador.

Quiñones, M., Miguel, M., \& Aleixandre, A. (2012). Los polifenoles, compuestos de origen natural con efectos saludables sobre el sistema cardiovascular. (1). https://doi.org/10.3305/nh.2012.27.1.5418

Ramli, N., Yatim, A. M., Said, M., \& Hok, H. C. (2001). HPLC Determination of Methylxanthines and Polyphenols Levels In Cocoa and Chocolate Products. Malaysian Journal of Analytical Sciences, 7(2), 377-386. 
Riaño, N., Chica, M., Echeverri, L., Aguirre, J., Ortiz, A., Pineda, R., \& Olarte, N. (2016). Contenido de grasa total, perfil de Ácidos grasos y triglicéridos proveniente de cacaos finos de aroma: Colombia, Ecuador, Perú, Venezuela (Vol. 23).

Ribeiro, P. V. de M., Andrade, P. A., Hermsdorff, H. H. M., dos Santos, C. A., Cotta, R. M. M., Estanislau, J. de A. S. G., ... Rosa, C. de O. B. (2019). Dietary nonnutrients in the prevention of non-communicable diseases: Potentially related mechanisms. Nutrition, 66, 22-28. https://doi.org/10.1016/j.nut.2019.03.016

Rodríguez-Carrasco, Y., Gaspari, A., Graziani, G., Santini, A., \& Ritieni, A. (2018). Fast analysis of polyphenols and alkaloids in cocoa-based products by ultra- high performance liquid chromatography and Orbitrap high resolution mass spectrometry ( UHPLC-Q-Orbitrap-MS / MS ). Food Research International, 111(February), 229-236. https://doi.org/10.1016/j.foodres.2018.05.032

Romanens, E., Freimüller Leischtfeld, S., Volland, A., Stevens, M., Krähenmann, U., Isele, D., ... Miescher Schwenninger, S. (2019). Screening of lactic acid bacteria and yeast strains to select adapted anti-fungal co-cultures for cocoa bean fermentation. International Journal of Food Microbiology, 290(October 2018), 262272. https://doi.org/10.1016/j.ijfoodmicro.2018.10.001

Saltini, R., Akkerman, R., \& Frosch, S. (2013). Optimizing chocolate production through traceability: A review of the in fl uence of farming practices on cocoa bean quality. Food Control, 29(1), 167-187. https://doi.org/10.1016/j.foodcont.2012.05.054

Samaniego, I., Espín, S., Quiroz, J., Ortiz, B., Carrillo, W., García-Viguera, C., \& Mena, P. (2020). Effect of the growing area on the methylxanthines and flavan-3-ols content in cocoa beans from Ecuador. Journal OfFood Composition and Analysis, 1(January), 103448. https://doi.org/10.1016/j.jfca.2020.103448

Santana, P. (2017). Mucílago de cacao (Theobroma cacao I.), nacional y trinitario para la obtención de una bebida hidratante. Universidad Técnica Estatal de Quevedo.

Santos, D. S., Rezende, R. P., Santos, T. F. dos, Marques, E. de L. S., Ferreira, A. C. R., Silva, A. B. de C. e., ... Tavares Bisneto, J. D. (2020). Fermentation in fine cocoa type Scavina: Change in standard quality as the effect of use of starters yeast in fermentation. Food Chemistry, 328(October 2019), 7-12. https://doi.org/10.1016/j.foodchem.2020.127110

Sarbu, I., \& Csutak, O. (2019). The microbiology of cocoa fermentation. In Caffeinated and Cocoa Based Beverages: Volume 8. The Science of Beverages. 
https://doi.org/10.1016/B978-0-12-815864-7.00013-1

Schinella, G., Mosca, S., Cienfuegos-jovellanos, E., Ángeles, M., Muguerza, B., Ramón, D., \& Luis, J. (2010). Antioxidant properties of polyphenol-rich cocoa products industrially processed. Food Research International, 43, 1614-1623. https://doi.org/10.1016/j.foodres.2010.04.032

Serra, D., Almeida, L. M., \& Dinis, T. C. P. (2019). Polyphenols as food bioactive compounds in the context of Autism Spectrum Disorders: A critical mini-review. Neuroscience and Biobehavioral Reviews, 102(March), 290-298. https://doi.org/10.1016/j.neubiorev.2019.05.010

Servent, A., Boulanger, R., Davrieux, F., Pinot, M. N., Tardan, E., Forestier-Chiron, N., \& Hue, C. (2018). Assessment of cocoa (Theobroma cacao L.) butter content and composition throughout fermentations. Food Research International, 107(2017), 675-682. https://doi.org/10.1016/j.foodres.2018.02.070

Silva, R. F. M., \& Poga“cnik, L. (2020). Polyphenols from food and natural products: Neuroprotection and safety. (2010), 1-13.

Sirbu, D., Corno, M., Ullrich, M. S., \& Kuhnert, N. (2018). Characterization of triacylglycerols in unfermented cocoa beans by HPLC-ESI mass spectrometry. Food Chemistry, 254(February), 232-240. https://doi.org/10.1016/j.foodchem.2018.01.194

Steinberg, F. M., Bearden, M. M., \& Keen, C. L. (2003). Cocoa and chocolate flavonoids: implications for cardiovascular health. Journal of the American Dietetic Association, 103(2), 215.

Sudano, I., Flammer, A. J., Roas, S., Enseleit, F., Ruschitzka, F., Corti, R., \& Noll, G. (2012). Cocoa , Blood Pressure, and Vascular Function. Current Hypertension Reports, 14, 279-284. https://doi.org/10.1007/s11906-012-0281-8

Szajdek, A., \& Borowska, E. J. (2008). Bioactive compounds and health-promoting properties of Berry fruits: A review. Plant Foods for Human Nutrition, 63(4), 147153. https://doi.org/10.1007/s11130-008-0097-5

Teye, E., Huang, X., Sam-amoah, L. K., Takrama, J., Boison, D., Botchway, F., \& Kumi, F. (2015). Estimating cocoa bean parameters by FT-NIRS and chemometrics analysis. Food Chemistry, 176, 403-410. https://doi.org/10.1016/j.foodchem.2014.12.042

Tezara, W., De Almeida, J., Valencia, E., Cortes, J. L., \& Bolaños, M. J. (2015). Actividad fotoquímica de clones élite de cacao ( $d$ L.) en el norte de la provincia de 
Esmeraldas. Investigación y Saberes, 4(3), 35-72.

Tezara, Wilmer, Urich, R., Jaimez, R., Coronel, I., Araque, O., Azócar, C., \& Chacón, I. (2016). Does griollo cocoa have the same ecophysiological characteristics as forastero? Botanical Sciences, 94(3), 563-574. https://doi.org/10.17129/botsci.552

Thuy, V. T., Zhao, J., \& Fleet, G. (2015). The effect of lactic acid bacteria on cocoa bean fermentation. International Journal of Food Microbiology, 205(May), 54-67. https://doi.org/10.1016/j.jifoodmicro.2015.03.031

Todorovic, V., Radojcic, I., Todorovic, Z., Jankovic, G., Dodevska, M., \& Sobajic, S. (2015). Journal of Food Composition and Analysis Polyphenols, methylxanthines, and antioxidant capacity of chocolates produced in Serbia. Journal of Food Composition and Analysis. https://doi.org/10.1016/j.jfca.2015.01.018

Torres-Moreno, M., Torrescasana, E., Salas-Salvadó, J., Blanch, C., Torres-Moreno, M., Torrescasana, E., ... Blanch, C. (2015). Nutritional composition and fatty acids profile in cocoa beans and chocolates with different geographical origin and processing conditions. Food Chemistry, 166, 125-132. https://doi.org/10.1016/j.foodchem.2014.05.141

Torres Murillo, M. (2016). Cadena de valor para la comercialización de cacao de los productores del recinto Tazone (UNIVERSIDAD CATÓLICA DEL ECUADOR SEDE ESMERALDAS DIRECCIÓN; Vol. https://doi.org/10.1017/CBO9781107415324.004

Vargas Jentzsch, P., Ciobotă, V., Salinas, W., Kampe, B., Aponte, M., Rösch, P., Ramos, L. A. (2016). Distinction of Ecuadorian varieties of fermented cocoa beans using Raman spectroscopy. Food Chemistry, 211, 274-280. https://doi.org/10.1016/j.foodchem.2016.05.017

Vera Chang J, Cabrera Verdezoto R, Veliz Zamora D, Segovia Freire G, Torres Navarrete Y, Pinargote Alava J, . (2018). Validación de tres métodos de propagación en cacao (Theobroma cacao L.) Nacional y Trinitario en Ecuador. Conamti, 5(01), 01-06.

Wang, G., Lee, A. S., Lewis, M., Kamath, B., \& Archer, R. K. (1999). Accelerated solvent extraction and gas chromatography/mass spectrometry for determination of polycyclic aromatic hydrocarbons in smoked food samples. Journal of Agricultural and Food Chemistry, 47(3), 1062-1066.

Wollgast, J., \& Anklam, E. (2000). Review on polyphenols in Theobroma cacao: changes in composition during the manufacture of chocolate and methodology for 
identification and quantification. Food Research International, 33.

Wood, G. A. R., \& Lass, R. A. (1985). Cocoa (4th Editio; Longman, Ed.). London, United Kingdom.

Yépez Rivadeneira, J. (2017). Caracterización del contenido de polifenoles: catequina, epicatequina y procianidinas $\mathrm{B} 1, \mathrm{~B} 2$ y $\mathrm{C} 1$; en cacao $\mathrm{C} C \mathrm{~N}-51$ de las principales zonas productoras del Ecuador. UNIVERSIDAD CENTRAL DEL ECUADOR.

Zambrano, G. (2018). Evaluación de la influencia del proceso de beneficio del cacao (Theobroma cacao) CCN-51 de altura en su calidad final, mediante el análisis físico, físico-químico y sensorial. UNIVERSIDAD CENTRAL DEL ECUADOR.

Zambrano Tenezaca, J. (2017). Caracterización físico química y nutricional de bebida de tomate de árbol con inclusión de cascarilla de cacao. Universidad Técnica de Machala.

Żyżelewicz, D., Wiesława, K., Oracz, J., Sosnowska, D., Grażyna, B., \& Nebesny, E. (2016). The in fl uence of the roasting process conditions on the polyphenol content in cocoa beans, nibs and chocolates. Food Research International Journal, 89, 918-929. https://doi.org/10.1016/j.foodres.2016.03.026 